\title{
Do your eyes protect your memory?
}

Citation for published version (APA):

Houben, S. (2021). Do your eyes protect your memory? From memory myths to the false memory potential of Eye Movement Desensitization and Reprocessing. [Doctoral Thesis, Maastricht University, KU Leuven]. Optima Grafische Communicatie. https://doi.org/10.26481/dis.20210527sh

\section{Document status and date:}

Published: 01/01/2021

DOI:

10.26481/dis.20210527sh

Document Version:

Publisher's PDF, also known as Version of record

\section{Please check the document version of this publication:}

- A submitted manuscript is the version of the article upon submission and before peer-review. There can be important differences between the submitted version and the official published version of record.

People interested in the research are advised to contact the author for the final version of the publication, or visit the DOI to the publisher's website.

- The final author version and the galley proof are versions of the publication after peer review.

- The final published version features the final layout of the paper including the volume, issue and page numbers.

Link to publication

\footnotetext{
General rights Owners
rights.

- You may freely distribute the URL identifying the publication in the public portal. please follow below link for the End User Agreement:

www.umlib.nl/taverne-license

Take down policy

If you believe that this document breaches copyright please contact us at:

repository@maastrichtuniversity.nl

providing details and we will investigate your claim.
}

Copyright and moral rights for the publications made accessible in the public portal are retained by the authors and/or other copyright owners and it is a condition of accessing publications that users recognise and abide by the legal requirements associated with these

- Users may download and print one copy of any publication from the public portal for the purpose of private study or research.

- You may not further distribute the material or use it for any profit-making activity or commercial gain

If the publication is distributed under the terms of Article $25 \mathrm{fa}$ of the Dutch Copyright Act, indicated by the "Taverne" license above, 


\section{Do your eyes protect your memory?}

From memory myths to the false memory potential of Eye Movement Desensitization and Reprocessing

Sanne Tessa Louise Houben 
(C) Sanne T. L. Houben, Maastricht, the Netherlands, 2021.

All rights reserved. No part of this book may be reproduced, stored in a retrieval system of any nature, or transmitted in any form or by any means (electronic, mechanical, photocopying, recording or otherwise) without prior written permission of the author, or when appropriate, by the publishers of the publications.

ISBN: $\quad 978-94-6361-508-2$

Cover art: Design by Anouk

Layout: $\quad$ Thomas H. P. M. Habets

Figures: $\quad$ Thomas H. P. M. Habets and Sanne T. L. Houben

Production: Optima Grafische Communicatie, Rotterdam, the Netherlands 


\title{
Do your eyes protect your memory?
}

\author{
From memory myths to the false memory potential of \\ Eye Movement Desensitization and Reprocessing
}

\section{PROEFSCHRIFT}

Ter verkrijging van de graad doctor in criminologie aan KU Leuven, België, op gezag van Rector Prof. Dr. Luc Sels en de graad doctor in psychologie aan de Universiteit Maastricht, op gezag van de Rector Magnificus, Prof. Dr. Rianne M. Letschert volgens het besluit van het College van Decanen, in het openbaar te verdedigen op 27 mei 2021 om 16:00 uur.

door

Sanne Tessa Louise Houben 


\section{Promotoren}

Prof. Dr. Henry Otgaar, Katholieke Universiteit Leuven en Maastricht University Prof. Dr. Harald L. G. J. Merckelbach, Maastricht University

\section{Co-promotor}

Dr. Jeffrey Roelofs, Maastricht University

\section{Beoordelingscommissie}

Prof. Dr. Corine de Ruiter (Voorzitter), Maastricht University

Prof. Dr. Agnes van Minnen, Radboud University

Prof. Dr. Steffen Moritz, Universitätsklinikum Hamburg-Eppendorf

Prof. Dr. Geert Vervaeke, Katholieke Universiteit Leuven 
"It does not take a scientist to understand what is going on.

If you see something in my eye, let us not overanalyze."

(Jennifer Paige - Crush, 1998)

Is EMDR just a little crush? 



\section{TABLE OF CONTENTS}

List of abbreviations

Chapter 1 General Introduction

Section I

Chapter 2 Metaphoric instructions in Eye Movement Desensitization and

Reprocessing

Chapter 3 The effects of eye movements and alternative dual tasks on the vividness and emotionality of autobiographical memory: A metaanalysis of laboratory studies

\section{Section II}

Chapter 4 Eye Movement Desensitization and Reprocessing practitioners' beliefs about memory

Chapter 5 Eye movements and suggestion-induced false memories

Chapter 6 Eye movements and spontaneous false memories

Chapter $7 \quad$ General Discussion

Summary

Samenvatting

Impact

Appendices

References

Dankwoord

Curriculum Vitae

List of publications 



\section{LIST OF ABBREVIATIONS}

AAT

(TF) CBT

CSA

EMDR

FTT

NEQ

PTSD

RCT

SMF

UE - ATR

WM
Associative Activation Theory

(Trauma Focused) Cognitive Behavior Therapy

Childhood Sexual Abuse

Eye Movement Desensitization and Reprocessing

Fuzzy Trace Theory

Negative Events Questionnaire

Post-Traumatic Stress Disorder

Randomized Controlled Trial

Source Monitoring Framework

Unwanted Event - Adverse Treatment Reactions

Working Memory 


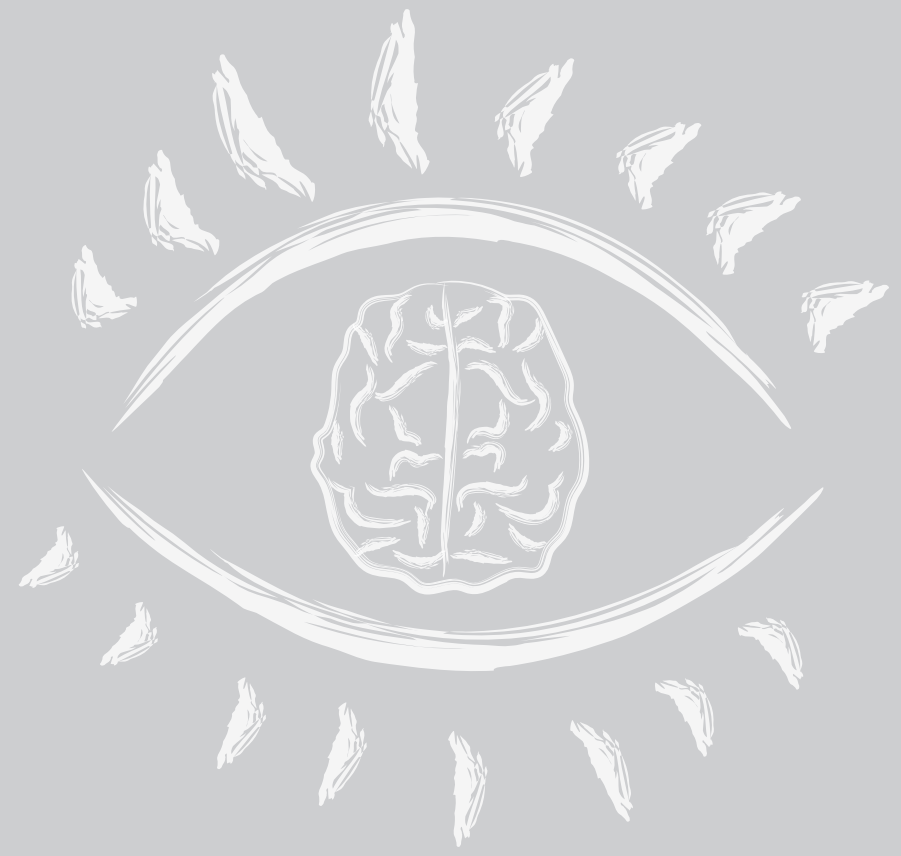




\section{CHAPTER 1}

\section{General Introduction}

This chapter is an extended and combined version of the following publications:

Otgaar, H., \& Houben, S. T. L. (in press). Pseudoherinneringen [False memories]. In: H. Otgaar, H. Merckelbach, \& M. Jelicic (Eds.), Rechtspsychologie. Zutphen: Uitgeverij Paris B. V.

Otgaar, H., Houben, S. T. L., \& Howe, M. L. (2019). Methods of studying false memory. In: B. L. Schwartz \& H. Otani, (Eds.), Research methods in human memory research (pp. 238250). New York, NY: Routledge. 

In 2017, Emma ${ }^{1}$, 27-years-old, repeatedly sought help for mental health complaints. She had feelings of anxiety, felt depressed, had trouble sleeping and experienced nightmares, had no energy, and was dealing with a divorce. She wanted an answer about the cause of her complaints and reasoned that therapy could help her find this cause.

According to her therapist, her complaints met the symptoms of post-traumatic stress disorder, although Emma had no memory of a specific traumatic incident. Most individuals experience at least one traumatic event at some point in their lives (Benjet et al., 2016). Experiencing a traffic accident, sudden loss of a relative or close friend, or sexual abuse are all examples of traumatic incidents that someone might suffer from in their life. These traumatic incidents are likely to affect one's mental health and daily functioning. If an individual is not able to cope with the traumatic incident, acute stress disorder emerges. If these symptoms persist over time, an individual might develop post-traumatic stress disorder (PTSD).

The therapist decided to target a vague childhood memory of a family trip by using Eye Movement Desensitization and Reprocessing (EMDR; Shapiro, 2002) as a therapeutic intervention. After several EMDR sessions, Emma recalled vivid memories of sexual abuse by her uncle. She did not have these memories before therapy. The therapist continued the EMDR therapy, until Emma's distress on this image reached zero. Emma attributed her symptoms to this traumatic memory and felt better after completing EMDR therapy.

Emma decided to confront her uncle about the alleged sexual abuse memory she discovered during therapy. Her uncle denied, but Emma did not believe him. She decided to file an official complaint against her uncle and the case entered the legal arena. As in most cases like these, no objective evidence regarding the alleged sexual abuse is present. Therefore, the judge had to solely rely on Emma's statements. A central question that arose concerned the reliability of her statement: how authentic is the memory of alleged sexual abuse? Or could it be that the memory is the result of the treatment intervention?

\section{Recovered Memory Debate}

Emma is a canonical example of a case in which the validity of a memory is questioned by the court. Cases like these were at the centre of a heated debate within the psychological field and beyond. That is, in the beginning of the 1990s, many individuals claiming to have recovered memories of childhood sexual abuse (CSA) during therapy filed an official complaint with the police, which (in some cases) led to legal proceedings. At that time, the authenticity of these

${ }^{1}$ Fictitious name for privacy reasons, case derived from clinical practice. 


\section{Chapter 1}

traumatic memories was oftentimes questioned. Typically, individuals received psychological therapy for other psychological complaints and at the start of treatment did not have any traumatic memories concerning CSA. However, after multiple therapeutic sessions, they started to recover memories about the abuse (Loftus, 1993). Two main positions characterized this debate: the repressed memory account versus the false memory account.

Repressed Memory Account. Many clinicians asserted that the recovery of such memories took place because the memory of the traumatic event was initially repressed and by means of therapy could be accurately retrieved (i.e., the repressed memory account; Brown, Scheflin, \& Whitfield, 1999). The idea here was that special defence mechanisms blocked traumatic memories out of consciousness (Spiegel, 1997). As a result, individuals were incapable to remember the traumatic event, until it was psychologically safe for them to do so. This would happen automatically and selectively: only the most traumatic memories would be blocked. If it was safe for the individual to recall the traumatic memory, the memory itself would be completely intact (Crews, 1995; Wessel et al., 2017). This view has its origin in the work of Freud (1954). Evidence for repression comes from retrospective memory studies. For example, Briere and Conte (1993) surveyed 450 adults who said to have memories of abuse. Some of the participants $(59.3 \%, n=267)$ claimed to have no memories of the abuse for a certain period of time. A major drawback of such studies is the lack of validation of the abuse and lack of assessment of how the memory was retrieved (Loftus \& Davis, 2006).

Many academics, on the other hand, stated that traumatic experiences are generally well remembered (Goldfarb et al., 2019; Holland \& Kensinger, 2010; McNally, 2003; Peace \& Porter, 2004). For example, from scientific case studies (see e.g., Bidrose \& Goodman, 2000; Leander, Christianson, \& Granhag, 2007; Goodman et al., 2003; Orbach \& Lamb, 1999; Weede Alexander et al., 2005), it becomes clear that traumatic events are not repressed but well recollected. In such studies, victims' statements could be compared with objective evidence (e.g., audio, photo, or video material) of the abuse as well as witness and/ or perpetrator statements. The victims' statements were highly accurate (around $80 \%$ ) and most of the details pertained to central elements (i.e., related to the sexual abuse). Details that were omitted by the victims were often a result of feelings of shame, not because they were unable to remember.

False Memory Account. Many memory scholars argued that memories recovered in therapy do not reflect authentic events, but might be false memories (i.e., memories of events/details that the individual did not experience; Loftus, 2005). For example, in some 
cases, they argued that the therapist believed in the concept of repressed memories and perhaps falsely suggested that the patient experienced CSA. Studies have found evidence to suggest that (im)plausible events can be implanted in individuals' minds, such as ritual abuse (Shaffer \& Cozolino, 1992) and alien abduction (Otgaar, Candel, Merckelbach, \& Wade, 2008; this study included children). Furthermore, a number of individuals who initially recovered memory of sexually abuse retracted their claims (Loftus \& Davis, 2006). Several studies examined how these patients created and later retracted their traumatic memories (De Rivera, 1997; Lief \& Fetkewicz, 1995; Ost, Costall, \& Bull, 2001, 2002). The majority of these individuals was in therapy for depression and recovered their memories of abuse during therapy. When they expressed their doubts concerning the authenticity of these memories, their therapists explained that such doubt is common (Ost et al., 2002). Reasons to retract their claims were related to the quality of the memory: it was either too clear and vivid or too vague and dreamlike (Lief \& Fetkewicz, 1995; Ost et al., 2002).

This does not mean that all traumatic memories recovered in therapy are false memories. Two types of recovered memories have been identified (Schooler, 2001). The first type concerns traumatic memories that are gradually recovered during therapy. Oftentimes the recovery is assisted by means of suggestive therapeutic interventions (e.g., hypnosis, guided imagery; Lilienfeld, 2007). The second type refers to spontaneous recovered memories that take the form of a sudden recall of traumatic memories outside a therapeutic context. Here, it is possible that confrontation with a stimulus that is closely linked to the abuse (contextual reinstatement; for example, exposure to a playground), remind the individual of the traumatic event (e.g., abused in the playground). Individuals with spontaneous recovered memories tend to underestimate prior recall attempts of the traumatic event (Brenneis, 2000; Fivush \& Edwards, 2004; Merckelbach et al., 2006, Study 3; Raymaekers, Peters, Smeets, Abidi, \& Merckelbach, 2011). To illustrate this, in one experiment, 15 individuals who claimed they had recovered memories of CSA had to indicate how vivid their memories were about presented childhood events (Merckelbach et al., 2006). After an hour, they completed another questionnaire that contained a few items that were presented to them in the first questionnaire. In this second questionnaire, they were asked when they recalled the specific childhood event (e.g., one hour or less ago or several years ago). The recovered memory individuals erroneously claimed that the last time they recalled the event was a year ago. This finding suggests that they tended to overlook recent recall attempts. However, one cannot state that memories recovered during therapy are, per definition, false memories. 


\section{Chapter 1}

A third interpretation proposed the idea that some recovered memories can reflect truly experienced events.

Third Interpretation. Because the repressed memory side of the debate lacks scientific foundation, and the false memory account could not explain recovered memories that are authentic, a third interpretation has been postulated (McNally \& Geraerts, 2009). This account attempts to explain the emergence of authentic memories without ignoring the possible existence of false memories. For example, in some cases, individuals might not have experienced the traumatic event as traumatic at the time when the trauma occurred (Clancy, 2010; Loftus \& Davis, 2006). The lack of traumatic impact at the time, but realization of the impact later in life, might be the reason why individuals come to believe in repressed memories. This lack of impact might be due to lack of understanding when the event happened during childhood, because the individual was simply too young to understand what truly happened. Hence, the event was not experienced as traumatic. Due to reminders (e.g., contextual cues) later in life, the memory can come to mind. The recollection can be accompanied by shock, because now the individual is able to understand the impact of the event, which leads to a reinterpretation of the event (Raymaekers, Smeets, Peters, Otgaar, \& Merckelbach, 2012).

Some authors who were sceptical of repressed memories claimed that the recovered memory debate was over (e.g., Barden, 2016; McHugh, 2003; Paris, 2012). However, there are several strong indicators that this debate is alive and well, in clinical, legal and academic settings (Otgaar, Howe, Patihis, et al., 2019). For example, recent survey studies have indicated that may clinicians continue to believe in the controversial topic of repressed memories (Patihis, Ho, Tingen, Lilienfeld, \& Loftus, 2014; Ost, Wright, Easton, Hope, \& French, 2013; Otgaar, Howe, Patihis, et al., 2019). A therapist who has strong beliefs in the existence of repressed memories can suggestively search for such memories in patients. However, human memory (including traumatic memory) is reconstructive (McNally, 2005). This means that during every retrieval attempt, memory errors may arise and as a result, memory changes even for negative and traumatic experiences. Such experiences play a central role for patients diagnosed with post-traumatic stress disorder.

\section{Posttraumatic Stress Disorder}

Following the fifth edition of the Diagnostic and Statistical Manual of Mental Disorders (DSM5; American Psychiatric Association, 2013), eight criteria must be met for an individual to 
be classified with posttraumatic stress disorder (PTSD). Criterion A states that the individual was exposed to a life threatening or traumatic event. Furthermore, patients with PTSD are thought to suffer from memory-related symptoms such as intrusions (i.e., recurrent, involuntary, and distressing recollections of the traumatic event) and flashbacks (i.e., dissociative reactions in which the individual feels as if the traumatic event is recurring) after exposure to trauma (Criterion B). Also, trauma related stimuli are avoided (Criterion C) and the individual experiences negative cognitions and emotional numbing (e.g., "It is all my fault"; Criterion D). The individual has alterations in arousal (e.g., irritability) and reactivity (e.g., hypervigilance; Criterion E). Lastly, the symptoms must last for more than one month (Criterion F), the symptoms impair daily functioning (Criterion $\mathrm{G}$ ), and are not caused by medication, substance use, or other illness (criterion $\mathrm{H}$ ).

As implied by the DSM-5 criteria, patients with PTSD experience memory problems. They tend to have a bias towards enhanced recall of the traumatic event (i.e., intrusions; Buckley, Blanchard, \& Neill, 2000). These memories can be vivid and long lasting (e.g., Brown \& Kulik, 1997; Rubin \& Kozin, 1984) or vague, lacking details and error prone (e.g., Koss, Figueredo, Bell, Tharan, \& Tromp, 1996; Loftus \& Burns, 1984). As described above, another notable memory related symptom of PTSD are flashbacks. These are dominated by sensory details (e.g., vivid images or sounds). The traumatic event seems to be unfolding in the present, rather than in the past.

One of the most popular interventions to treat PTSD is Eye Movement Desensitization and Reprocessing. Patihis and Pendergrast (2019) found that some individuals who received EMDR therapy indicated that their therapist discussed repressed memories with them. But what do we know about this intervention? And can it be the case that EMDR increases the risk to produce memories of events/details that the individual did not experience (i.e., false memories; Loftus, 2005)? Below, I will explain the phenomenon of false memory and EMDR in more depth.

\section{False Memory Formation}

Our memory plays a critical role in our daily life. Our memory helps us in remembering names, remembering plans for the day, recalling our grocery list, etc. However, not all memories are, per definition, authentic. Individuals can produce false memories (i.e., memories of events/ details that the individual did not experience; Loftus, 2005) as well. Arguably, false memories can have practical implications for the legal field. For example, false memories can have 


\section{Chapter 1}

serious consequences when they appear in the testimony of an eyewitness or victim (Howe \& Knott, 2015). Specifically, an individual might falsely remember that she ${ }^{2}$ was sexually abused when she was a child, and such false memories might lead to false accusations that may result in wrongful convictions (Saks \& Koehler, 2005).

Because of the legal implications that such false memories might have, a plethora of studies have been devoted to investigate the creation and factors underlying the creation of false memories. Across these studies, a wide variety of methods, also called (experimental) paradigms, have been used to (experimentally) induce false memories in a laboratory setting. However, limited evidence has been found that performance on one false memory task (e.g., DRM word lists) predicts performance on another false memory task (e.g., misinformation acceptance; Bernstein, Scoboria, Desjarlais, \& Soucie, 2018; Patihis, Frenda, \& Loftus, 2018). Furthermore, individual differences (i.e., personality traits) do not seem to strongly predict memory susceptibility across these tasks. It was for this reason that Patihis (2018) argues that there is no specific false memory trait, but rather that all individuals are prone to create false memories.

\section{Paradigms to Study Suggestion-Induced False Memories}

Because of the suggested sexual abuse claims during the recovered memory debate, memory researchers have examined how suggestion can shape memory. In the false memory literature, several paradigms have been developed to gain insight into the formation of suggestion-induced false memories. These paradigms aim to study (1) the incorporation of incorrect details (i.e., misinformation paradigm), (2) the integration of complete fictitious events (i.e., implantation paradigm); and (3) the influence of peers/authorities (rumormongering paradigm). As the first two aims (and paradigms) are most relevant for the current dissertation, they will be discussed in detail below.

Misinformation Paradigm. The misinformation paradigm (Loftus, 2005; Loftus, Miller, \& Burns, 1978) employs a three-phased procedure. In the first phase, participants witness a mock crime or view a video about a theft. Hereafter, during the misinformation phase, participants are presented with misinformation in the form of a narrative or suggestive questions (e.g., asking what weapon the culprit was carrying, even though there was no weapon present). In the final phase, participants take a memory test in which they are asked what they can remember about the stimuli. A significant minority of participants fall prey to the misinformation and incorporate it in their memory reports. This is known 
as the misinformation effect (Loftus, 2005; Frenda, Nahleen, Strange, \& Takarangi, 2020; Nichols, \& Loftus, 2011; Zaragoza \& Mitchell, 1996; Zaragoza, Belli, \& Payment, 2006). The misinformation effect can lead to poorer memory for the original experienced event and/ or incorporation of misinformation into the original event memory (Blank \& Launay, 2014; Leding \& Antonie, 2019).

In one of the first demonstrations of the misinformation effect, Loftus and colleagues (1978) presented slides of a car accident to their participants. Participants saw a slide in which a red car stopped at a stop sign. In a follow-up questionnaire, half of the participants were asked whether another car passed the red car while it stopped at the stop sign. For the other half of participants, the stop sign was replaced with a yield sign. Thus, these participants received misinformation. In a final recognition test, participants were presented with pairs of slides and were asked to indicate which slide they saw. More than half (59\%) of the misinformed participants remembered the wrong slide (i.e., yield sign), against 25\% of the control participants, thereby demonstrating a misinformation effect (see also McCloskey \& Zaragoza, 1985).

Time seems to be an important factor of misinformation acceptance. Misinformation is more likely to be accepted after a period of time (Loftus et al., 1978). The memory of the original event weakens over time and as a result, it is likely that the misinformation is not noticed. In addition, the longer the interval between the presentation of misinformation and the memory test, the more likely it is for misinformation to be reported (Higham, 1998).

In general, young children are more susceptible to the misinformation effect than older children and adults (Ceci \& Bruck, 1993; but see Otgaar, Howe, Merckelbach, \& Muris, 2018). In addition, reporting of misinformation has been shown to be negatively related to intelligence in some studies (i.e., higher IQ scores were related with less reporting of misinformation; Eisen, Quas, \& Goodman, 2002; Zhu et al., 2010), while others could not find such associations (Powers, Andriks, \& Loftus, 1979; Salthouse \& Siedlecki, 2007). Poor memory capacity may also lead to increased susceptibility as source memory errors (see below) are more likely to occur (e.g., Jaschinski \& Wentura, 2002; Peters, Jelicic, Verbeek, \& Merckelbach, 2007; Zhu et al., 2010). Perceptual talents (i.e., stronger abilities to imagine visual images) might be an explanation for the endorsement of misinformation. Drivdahl and Zaragoza (2001) presented a video of a burglary to their participants. A week later, participants read a narrative of the scenes they had seen containing misinformation and took a memory test. Some of the participants were asked to elaborate on perceptual details of the scenes. 


\section{Chapter 1}

Two days later, they underwent a source memory test. Participants who were encouraged to elaborate on perceptual characteristics of the misinformed details were more likely to claim they remembered, for example, a ring being stolen, while no jewelry was taken (see also Cann \& Katz, 2005; Eisen, Gomes, Lorber, Perez, \& Uchishiba, 2013; Tomes \& Katz, 1997).

Misinformation can have disastrous consequences in the legal arena. Not only can it alter memory for details, it can also distort memory of faces (Morgan, Southwick, Steffian, Hazlett, \& Loftus, 2013), which can influence lineup performance (Cochran, Greenspan, Bogart, \& Loftus, 2016). Consequently, an innocent individual might be identified as the perpetrator, thereby contributing to false accusations (Loftus, Doyle, \& Dysart, 2013). Though the misinformation paradigm elicits subtle false memories of details, autobiographical false memories of entire events can also be suggestively elicited.

Implantation Paradigm. The implantation paradigm can be seen as an extension of the misinformation paradigm. In the implantation paradigm, entire fictitious events are inserted into memory (Hyman \& Billings, 1998; Hyman, Husband, \& Billings, 1995; Loftus \& Pickrell, 1995). Specifically, in a typical implantation experiment, participants are interviewed about some childhood events. Unknown to the participants is that the experimenter has fabricated one of these childhood events. This childhood event is checked with the participant's parent(s) to confirm that the event is, indeed, not part of the autobiography of the participant. Participants are suggestively informed that their parents told the researchers about these events. Over the course of multiple interviews, the participants attempt to recall everything they remember about these events. Scoboria and colleagues (2017) used a scoring system to code memory transcripts of eight implantation studies (total $N=423$ ). Using this coding system, $30.4 \%$ of the reports were found to be vulnerable to these suggestions; here, participants falsely remembered the nonexperienced event as happening to them in their childhood (Scoboria et al., 2017).

Loftus and Pickrell (1995) were the first attempting to implant false memories in participants. In their experiment, they suggestively interviewed participants about the false event that they were lost in a shopping mall when they were five years old. A quarter of participants $(n=6)$ created implanted false memories for the suggested event and provided additional event-related details. To implant false memories, other studies have used fake photographs of, for example, a hot air balloon ride (Wade, Garry, Read, \& Lindsay, 2002).

Several factors can increase the acceptance of an implanted false memory. For example, the suggested event needs to be plausible. Pezdek, Finger, and Hodge (1997) 
presented their participants with two childhood events: lost in a shopping mall and receiving an enema. None of the participants claimed to have received an enema, whilst $15 \%(n=3)$ claimed to have been lost in a shopping mall when they were little. However, some researchers have been successful in implanting bizarre events (e.g., having a cup of tea with Prince Charles; Strange, Sutherland, \& Garry, 2006; a UFO abduction; Otgaar et al., 2009) with the help of pictures or fake news articles. Script knowledge (i.e., prior event knowledge; Fivush, 1997) seems to affect false beliefs (Scoboria, Mazzoni, Kirsch, \& Jimenez, 2006), though this is limited to how familiar a certain event is (Pezdek, Blandon-Gitlin, Lam, Hart, \& Schooler, 2006). The valence (i.e., emotional aspect) of the event also increases the likelihood to accept the suggested detail or event. The relationship between valence and false memories will be discussed below.

\section{Paradigm to Study Spontaneous False Memories}

False memories can also occur without external suggestions as is used in the paradigms described above. Such false memories are also called spontaneous false memories (Brainerd et al., 2008). The Deese/Roediger-McDermott paradigm is one of the most frequently used methods to induce spontaneous false memories.

Deese/Roediger-McDermott Paradigm. The Deese/Roediger-McDermott (DRM; Deese, 1959; Roediger \& McDermott, 1995) paradigm is often used to elicit spontaneous false memories. In a typical DRM study, participants read word lists containing associated words (e.g., blood, police, gun, etc.) that are all related to a non-presented word, also known as the critical lure (i.e., murder). Participants are instructed to memorize the words. Next, they are asked to freely recall words that were presented and/or are given a recognition test including presented, related, unrelated, and critical lure words. A robust finding is that many participants falsely remember and/or recognize the critical lure (Gallo, 2006; 2010). Instead of word lists, researchers have also used stories or videos with associatively related details (Dewhurst, Pursglove, \& Lewis, 2007; Otgaar, Howe, Peters, Sauerland, \& Raymaekers, 2013).

Regarding individual differences, it was argued that adults were less prone to spontaneous false memories than children (Balota et al., 1999; Butler, McDaniel, Dornburg, Price, \& Roediger, 2004; Gallo, Bell, Beier, \& Schacter, 2006; Kensinger \& Schacter, 1999). However, a developmental reversal effect has been found, showing that young children are less susceptible to create spontaneous false memories than older children and adults (Brainerd, Reyna, \& Ceci, 2008; Otgaar, Howe, Muris, \& Merckelbach, 2019). An explanation 


\section{Chapter 1}

for this effect is that children have less background knowledge and therefore make fewer associations between concepts. The older one gets, the more knowledge one obtains. As a result, the likelihood to form spontaneous false memories based on associations increases. As for suggestive false memories, the valence of stimuli also influences the production of spontaneous false memories.

\section{Valence and False Memories}

The majority of legal cases concern negative events, and therefore it is of utmost importance to understand whether the emotional aspect of an event (i.e., valence) can influence false memory formation. As described above, traumatic memory is reconstructive (McNally, 2005). In general, individuals pay more attention to negative than neutral events (Kaplan, Van Damme, Levine, \& Loftus, 2016). Therefore, negative events are well remembered compared with neutral events (Bidrose \& Goodman, 2000; Bradley, Greenwald, Petry, \& Lang, 1992; Cahill \& McGaugh, 1995; LaBar \& Phelps, 1998; Leander et al., 2007; Orbach \& Lamb, 1999; Talmi, Luk, McGarry, \& Moscovitch, 2007). Due to increasing arousal, attention narrows to central details at the expense of peripheral details (i.e., emotional memory narrowing; Christianson \& Loftus, 1991).

Negative Suggestion-Induced False Memories. For suggestion-induced false memories, negative misinformation has been shown to be more easily reported than neutral misinformation (Bookbinder \& Brainerd, 2016; Otgaar, Candel, \& Merckelbach, 2008; Van Damme \& Smets, 2014). For example, Porter, Spencer, and Birt (2003) presented negative, positive, or neutral photographs to their participants. Half of the participants were exposed to misleading questions. At the end of the study, participants had to take a recall test. Results demonstrated that negative stimuli enhanced misinformation reporting $(80 \%, n=24)$ compared with neutral $(40 \%, n=12)$ and positive $(40 \%, n=12)$ material.

Regarding implantation studies, Laney and Loftus (2008) were able to implant a negative memory of being hospitalized overnight and to have witnessed a violent fight. Shaw and Porter (2015) presented a false event (i.e., having engaged in a criminal act) to their participants. After three suggestive interviews, including imagination techniques and familial confirmation, the majority of the participants $(73 \%, n=44)$ claimed to remember the committed crime and were able to provide a detailed account. However, a different coding scheme was used in this study than usual, nor did the authors distinguish between false beliefs and memories. When the memory reports were re-examined, the $73 \%$ dropped to 30\% (Wade, Garry, \& Pezdek, 2018). 
Negative Spontaneous False Memories. As described above, spontaneous false memories are frequently examined by means of the DRM paradigm and memory is tested by free recall and/or recognition. Negative, arousing DRM lists have been shown to increase false recognition levels and decrease true recognition levels, compared with neutral DRM lists, whilst for false recall a reversed pattern is found (Bookbinder \& Brainerd, 2016; Brainerd, Holliday, Reyna, Yang, \& Toglia, 2010; Howe, Candel, Otgaar, Malone, \& Wimmer, 2010). Possible explanations (e.g., specific relation between free recall/recognition and gist/ verbatim/associative network) for this finding are unknown and in most studies recognition data has been confounded with prior recall.

\section{Theories on False Memories}

Several theories aim to explain the occurrence of false memories. The most common theories will be discussed below.

Discrepancy Detection. According to the discrepancy detection principle (Tousignant, Hall, \& Loftus, 1986; Loftus, 1992; Leding \& Antonio, 2019), if an individual does not detect discrepancies between misinformation and the original event, misinformation is more likely to be reported. The stronger the memory for the original event, the less likely it is for the misinformation to be reported, because it is easier to detect discrepancies between what was experienced and suggested. More time delay between the experienced and suggested event results in more discrepancy between the two events, thereby increasing the susceptibility to acceptance (Leding \& Antonio, 2019; Loftus, 1992).

Source Monitoring Framework. The Source Monitoring Framework (SMF; Johnson, Hashtroudi, \& Lindsay, 1993) is mainly used to explain the formation of suggestion-induced false memories. It has its origin in research on reality monitoring (Johnson, 1988; Johnson \& Raye, 1981; Johnson, Kahan, \& Raye, 1984). Reality monitoring refers to how an individual attributes a memory to an external or internal source (Johnson \& Raye, 1981). Memories that rely on external sources (i.e., obtained through perceptual processes, for example, determining which person said what) have more perceptual information, sensory and contextual information, whereas memories that rely on internal sources (i.e., reasoning, imagination, and thought processes) provide information on cognitive operations, for example, discriminating between thoughts and spoken ideas (Johnson \& Raye, 1981).

Source monitoring refers to the mechanisms involved in making inferences about the source of our memory. A memory can originate from an own experience, imagining an 


\section{Chapter 1}

event, watching an event (e.g., on TV), or listening to someone else's experience. Increases in similarities of memories from different sources, for example attributing a rude remark to friend $X$ while friend $Y$ was the one who made the claim, will decrease the accuracy of source monitoring (Johnson, 1991).

According to the SMF, suggestion-based false memories arise when sourcemonitoring errors occur. Source attributions are made very rapidly (Lindsay \& Johnson, 2000). Suggestive false memories occur when thoughts, images and/or feelings from one source are attributed to another, incorrect, source (Belli \& Loftus, 1994; Gerrie \& Garry, 2011; Mitchell \& Johnson, 2000; Zaragoza, Lane, Ackil, \& Chamber, 1997). This misattribution can occur when, for example, an imagined event is highly similar to an actual perceived event. For example, Johnson, Raye, Wang, and Taylor (1979) instructed participants to imagine a photo. The more times they performed this imagination, the more they thought they actually saw the photo (see also Henkel, Franklin, \& Johnson, 2000; Johnson, Foley, \& Leach, 1988).

Fuzzy Trace Theory. Fuzzy Trace Theory (FTT; Brainerd \& Reyna, 2002; Brainerd et al., 2008) postulates that individuals store memories along two traces: verbatim and gist traces. The verbatim trace includes surface components of experiences (e.g., seeing a golden retriever). The gist trace is involved in understanding the overall meaning of an experience (e.g., seeing a dog). Retrieval of verbatim traces results in suppressing the formation of false memories. Gist retrieval, on the other hand, induces the recollection of false events that share the same meaning with the original event (Brainerd et al., 2008).

The FTT predicts that the susceptibility to spontaneous false memories increases when verbatim traces are impossible to retrieve. Verbatim traces fade quickly over time and individuals then will rely on gist traces to retrieve memories. False memories will occur when gist traces lead to erroneous inferences (Brainerd et al., 2008).

Association Activation Theory. An alternative theory to explain the production of spontaneous false memories is the Association Activation Theory (AAT; Howe, Wimmer, Gagnon, \& Plumpton, 2009; Otgaar, Howe, Muris, \& Merckelbach, 2019). Similar to Activation Monitoring Theory (AMT; Roediger, Balota, \& Watson, 2001) and Semantic Network Theory (Collins \& Quillian, 1969), AAT derives its tenets from spreading activation models. That is, AAT stipulates that our memory consist of networks. During the encoding of an event, spreading activation will occur: the processing of a word activates a so-called node in one's knowledge base. Subsequently, this activation spreads to surrounding theme nodes (Collins \& Loftus, 1975). As a result, some nodes are activated because of their link with the presented items. 
These nodes were not presented, but related to the original event. The activation of such concepts can lead to the production of spontaneous false memories. Because knowledge increases with age, adults are more likely to make automatic associations and are more susceptible to create spontaneous false memories (Howe et al., 2009; Otgaar, Howe, Muris, \& Merckelbach, 2019). AAT postulates that an increase in true memories goes along with an increase in false memories.

Associative strength seems to play an important role in the formation of spontaneous false memories. The strength of the list items (e.g., bed, pillow, rest) to the critical lure (sleep) is known as backward associative strength (BAS; Howe et al., 2009). BAS and forward associative strength (FAS; the associative strength from critical lures to studied items) are herald to false memory production (Gallo \& Roediger, 2002; Roediger, Watson, McDermott, \& Gallo, 2001). The higher the BAS and FAS, the more likely it is that a false memory is created.

\section{Theories on PTSD}

Multiple theories have been put forward to explain how individuals develop PTSD. Older theories such as information processing theories (Foa, Steketee, \& Rothbaum, 1989) and the anxious apprehension model (Jones \& Barlow, 1990) have become obsolete due to accruing scientific evidence. More recent theories on PTSD will be discussed below.

Emotional Processing Theory. In this theory, pre-trauma knowledge plays a central role (Foa \& Riggs, 1993; Foa \& Rothbaum, 1998). For example, individuals who have fixed pre-trauma views would be more vulnerable to PTSD. These views could be positive (e.g., "I am extremely safe"), which would be contradicted by the traumatic event, or they could be negative (e.g., "The world is extremely dangerous"), which would be confirmed by the traumatic event.

Dual Representation Theory. Another influential view is that traumatic memories are stored along two memory systems. According to Brewin, Dalgleish, and Joseph (1996), these two memory systems operate in parallel. The verbally accessible memory (VAM) system contains information about the situation, the emotional reaction to it and the perceived meaning of the event and integration of the information within one's autobiographical memory (Brewin et al., 2010; Pearson et al., 2012). The VAM represents the conscious experience of the traumatic incident. The situational accessible memory (SAM) contains detailed information that comes from (unconscious) perceptual processing and bodily responses (e.g., heart rate) and is associatively organized. This system can be involuntary 


\section{Chapter 1}

triggered by internal or external cues (Brewin \& Holmes, 2003; Brewin, Gregory, Lipton, \& Burgess, 2010; Pearson, Ross, \& Webster, 2012). The SAM will be represented in detailed flashbacks.

Cognitive Model. According to the cognitive model of Ehlers and Clark (2000), individuals develop PTSD when the processing of the trauma leads to feelings of threat for future events, while the traumatic event lies in the past. This can result from negative appraisals (e.g., "nowhere is safe") and a strong sensory memory system and poor contextualisation memory system. These negative appraisals arise because of the thought processes during the traumatic events and prior beliefs about the event. It is argued that the emotional reaction during the trauma itself shifts from conceptual to sensory processing, which are called peritraumatic influences (see e.g., Bourne, Frasquilho, Roth, \& Holmes, 2010; Halligan, Michael, Clark, \& Ehlers, 2003). Conceptual processing focuses on the meaning of the situation, organizes the information, and aims to place it in context. This system integrates the traumatic memory within one's autobiographical memory. The sensory processing leads to perceptual priming and memories that are hard to retrieve intentionally (Ehlers \& Clark, 2000). Due to this shift during the traumatic event, eventually, intrusion frequency increases.

\section{PTSD and False Memories}

As described above, negative stimuli can enhance false memory susceptibility. Following the dual representation theory and the AAT, one could assume that due to reliance on the associatively organized sensory system, associative nodes could become incorrectly activated. As a result, an individual could remember an event as more traumatic than it originally has been. Strange and Takarangi (2012) examined this type of escalation and presented their participants with a car crash video. During a recognition test (including old, new and missing video clips), participants were asked to indicate whether they had seen the clips or not. Participants claimed to have seen missing clips. This distortion was stronger for traumatic than less traumatic details, indicating that they remembered more traumatic details than were actually present at the scene.

Results on the relationship between PTSD and false memory production are inconsistent. A link between PTSD and higher false memory levels was found in some studies (e.g., Moradi et al., 2015), but not in others (e.g., Dasse, Juback, Morisette, Dolan, \& Weaver, 2015). Goodman and colleagues (2011) presented participants who have a history of CSA with neutral, negative, and CSA-related DRM word lists. For free recall, negative and CSA DRM 
word lists resulted in the highest false recall levels. For false recognition, the opposite pattern surfaced. In addition, greater PTSD symptomatology was related to higher false recall levels. Otgaar, Muris, Howe, and Merckelbach (2017) looked at all false memory studies including participants with mental health problems (e.g., PTSD, history of trauma, depression) and found that PTSD was linked with increased false memory levels for negative DRM word lists when the words were associated to the experienced trauma.

\section{Treatment of PTSD}

A number of psychological interventions are available to treat PTSD. For example, traumafocused cognitive behavioral therapy is an intervention that uses cognitive and behavioral techniques and exposure approaches (see e.g., Cohen, Mannarino, \& Deblinger, 2017; Mavranezouli et al., 2020). Nowadays, one of the most popular interventions to treat PTSD is Eye Movement Desensitization and Reprocessing, which is the main focus of the current dissertation.

\section{Eye Movement Desensitization and Reprocessing}

Francine Shapiro (1989) discovered Eye Movement Desensitization (EMD) when she was walking through a park. Shapiro noticed that recurring, distressing thoughts were suddenly disappearing when her eyes were automatically moving from tree to tree. Shapiro speculated that the decrease of distressing thoughts arose because of the saccadic movement of her eyes while she held the distressing memory in her consciousness. Following this serendipitous discovery, Shapiro applied the EMD procedure in her own practice to patients with traumatic memories and the procedure seemed to successfully desensitize their traumatic memories.

EMDR Protocol. In subsequent years, EMD developed into Eye Movement Desensitization and Reprocessing (EMDR; Shapiro, 2002), which consists of a multiphasic intervention for the treatment of PTSD. The Dutch EMDR protocol (see Figure 1.1 and Appendix 1) differs slightly from Shapiro's original EMDR protocol (Hornsveld, ten Broeke, \& de Jongh, 2017a,b,c; Hornsveld, ten Broeke, \& de Jongh, 2018a,b). In general, in the first phases (phases $1-3$ ), the patient is screened and a treatment plan is prepared. The therapeutic relationship is established as well. The patient's expectations are set and the patient is informed about PTSD symptomatology and the treatment. The therapist and patient identify a target image (also called "hot spot") of the traumatic experience. This is an image where the patient experiences the highest level of distress (i.e., measured on the 


\section{Chapter 1}

subjective units of distress, SUDs; 0 = not distressful; 10 = very distressful). In the Dutch protocol, a therapist can choose between two metaphorical instructions (i.e., video or photo instruction) to identify the hot spot.

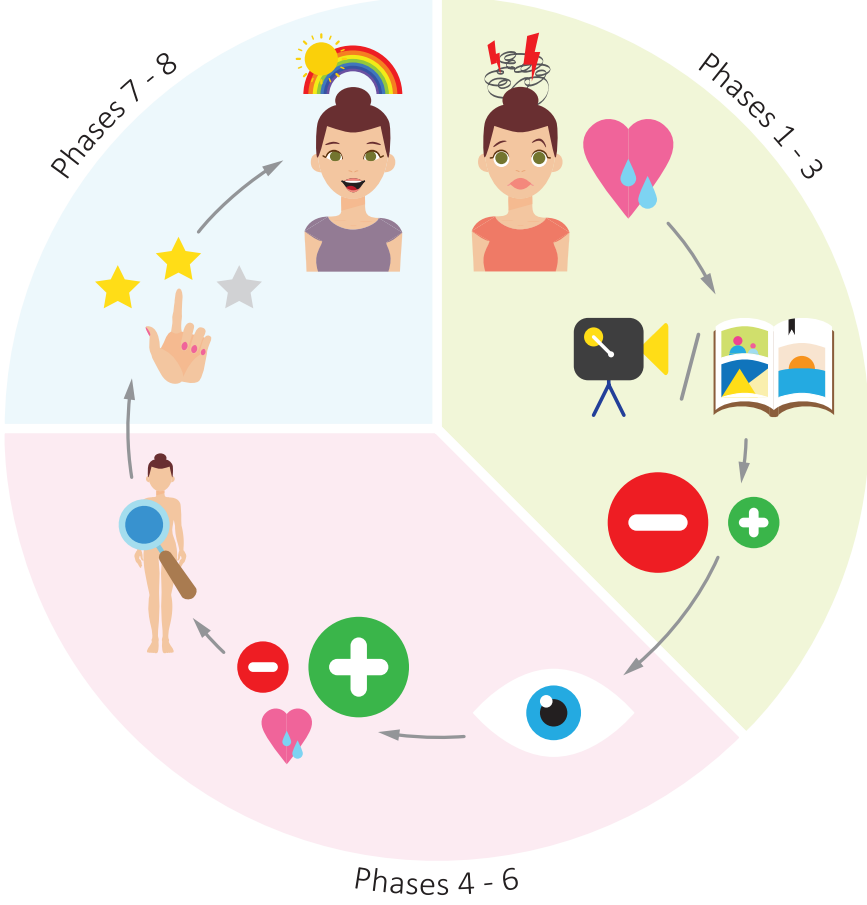

Figure 1.1 Phases of the Dutch EMDR protocol. In the first three phases, the target image and the negative and positive cognition are formulated. Phases $4-6$ are focused at the desensitization and reprocessing of the trauma by means of working memory taxation (e.g., eye movements). The negative cognition is weakened and the positive cognition is strengthened. In the last two phases, the session is evaluated.

Then, a cognition linked to this target image is identified. This cognition is hypothesized to be the reason why the traumatic memory is currently still distressing (de Jongh \& ten Broeke, 2016). Four main cognitions are available. Responsibility and guilt occurs when a patient feels guilty or has the feeling that he or she did not do enough at the time to prevent the event from happening. This cognition is oftentimes seen in victims of sexual abuse. Control is evident in victims of violent incidents and refers to a patient feeling helpless or powerless. Self-esteem refers to strong, believable, but incorrect, negative beliefs about oneself (e.g., "I am not worth it", "I am stupid"). This cognition is apparent in patients who 
experienced humiliating experiences (e.g., bullying, neglect). A patient can identify with the cognition Safety when he/she feels threatened and overestimates danger. This is seen in victims of, for example, car accidents.

Hereafter, the negative and positive cognitions are formulated. The negative cognition refers to a dysfunctional belief the patient has about him/herself when the target image is recalled. For example, for the cognition safety "I am in danger", or "I am a coward", the negative cognition has to be: (i) about the patient as a person; (ii) dysfunctional; (iii) linked to the target image; (iv) currently valid (i.e., the cognition is formed in present tense); (v) emotionally charged (de Jongh \& ten Broeke, 2016). After the negative cognition is established, the positive cognition is formulated. The positive cognition can be seen as the goal of the treatment. A positive cognition has to be (i) from a similar thematic content domain as the negative cognition; (ii) empowering; (iii) realistic and accessible; (iv) no history falsification (i.e., not altering the facts of the trauma/rewriting history); (v) about the patient; and (vi) without denials and references to the negative cognition. Examples of positive cognitions are: "I did what I could/I am safe/I am worth it". The credibility of the positive cognition is determined by the validity of cognition scale (VoC; 1 = completely unbelievable; $7=$ completely believable).

In the following phases (phases $4-6$ ), the desensitization and reprocessing take place. Here, the patient is asked to recall the target image and has to perform horizontal eye movements. The therapist initiates these eye movements by moving the index finger horizontally in front of the patient's visual field. Basically, the patient has to perform a dual task, given that (s)he needs to retrieve a target image and simultaneously perform eye movements. This procedure is repeated until the SUD that is elicited by the target image has decreased to zero. If the SUD does not decline, extra working memory taxation is offered (e.g., clicks by means of a headphone, buzzers holding in hands or fingers tapping on knees). Hereafter, the therapist aims to strengthen a positive cognition and to weaken the negative cognition related to the trauma. During this reprocessing, the patient has to perform the dual task once more. This step is repeated until the validity of cognition reaches full credibility (i.e., a score of 7). Finally, a so-called body scan is completed. Here, the patient is instructed to recall the target image and the positive cognition and to scan the body from head to toe. The body scan will expose any remaining bodily sensations related to the positive cognition (e.g., resistance).

During the last phases (phases $7-8$ ), a final evaluation of the therapeutic outcomes 


\section{Chapter 1}

will take place (e.g., "What is the most positive you have learned about yourself?"). This phase is especially important when the therapy itself is not yet completed. The goal is to create a positive state of mind (i.e., empowering state by means of an "I am..." statement) when leaving the therapist.

Effectiveness of EMDR. In the scientific community (see e.g., Friedman, 1996; Herbert et al., 2000; Lilienfeld, 1996; Lohr, Tolin, \& Lilienfeld, 1998; McNally, 1999; Muris \& Merckelbach, 1999), EMDR was viewed with scepticism. One reason for this was that it lacked any theoretical foundation. Randomized clinical trials and meta-analyses have, however, revealed that EMDR is effective in reducing the distress inherent to traumatic memories. More specifically, EMDR was superior to waitlist controls (Bisson \& Andrews, 2008; MorenoAlcázar et al., 2017; Wilson, Becker, \& Tinker, 1995) and was equally effective in treating PTSD compared with the generally accepted treatment cognitive behavioral therapy (Bisson et al., 2007; Moreno-Alcázar et al., 2017).

Though accepted in clinical practice, some controversy remained concerning the mechanisms underpinning the effectiveness of EMDR and whether performing eye movements was, in fact, necessary. Experimental studies examining the effect of EMDR typically focus on the eye movement component of the intervention. The canonical procedure in laboratory studies is that participants are instructed to retrieve negative autobiographical memories and to rate these memories on vividness and emotionality (pretest). Next, participants have to perform eye movements or not (i.e., memory recall-only) whilst simultaneously retrieving the negative memory. Afterwards, participants have to rate their memories on vividness and emotionality (post-test) once more. Lee and Cuijpers (2013) conducted a meta-analysis on how eye movements affected negative memories. Fifteen clinical trials and 11 laboratory studies that compared the standard EMDR procedure with eye movements against EMDR without eye movements were included. The clinical studies relied on the SUD and VoC measurements, as explained above. The laboratory studies relied on vividness and emotionality ratings. Overall, Lee and Cuijpers (2013) found that for clinical trials, the SUDs declined more when eye movements were included than when they were not. This between-treatment difference was associated with a medium effect size (Cohen's $d$ $=0.53)$. EMDR with eye movements also yielded greater increases in the VoC than a similar procedure conducted without eye movements (with a large effect size: Cohen's $d=0.72$ ). In a similar vein, the laboratory studies observed larger reductions in vividness and emotionality ratings of autobiographical memories following an EMDR procedure with eye movements as 
compared with a procedure without eye movements. Here, Cohen's ds were 0.91 and 0.66 , respectively, which can be interpreted as large and medium effect sizes (see also van den Hout \& Engelhard, 2012).

Cuijpers and colleagues (2020; see below) meta-analysed the therapeutic effects of EMDR and focused on short- and long-term decreases in symptomatology. The overall conclusion was that short-term beneficial effects are robust, but long-term effects are not. A recent network meta-analysis by Mavranezouli and colleagues (2020) analysed the effectiveness of several PTSD interventions (e.g., cognitive therapy, exposure therapy, EMDR). Although EMDR is thought to be superior to other effective treatments, no differences between EMDR and trauma focused cognitive behavioural therapy (TF-CBT) was found in reducing in PTSD symptoms (see also Bisson \& Andrews, 2008; Ehring et al., 2014).

Hypothesized Working Mechanisms of EMDR. A popular belief was that eye movements increase the communication between the left and right hemispheres of the brain (Gunter \& Bodner, 2008; Propper \& Christman, 2008). This way, EMDR would enhance the retrieval of trauma memories and associated content, which in turn would facilitate processing (Propper \& Christman 2008). As a result, the ability to remember a traumatic event without its negative emotions would improve. As long as the stimulus alternates between left and right, it does not matter whether the patient performs eye movements, tactile stimulation, or hears beeps. Gunter and Bodner (2008) tested this hypothesis by comparing horizontal eye movements with vertical eye movements. When participants recalled a negative memory and simultaneously performed horizontal eye movements, the well-known effect occurred: both vividness and emotionality ratings decreased. However, the same result was found when participants performed vertical eye movements, which contradicts the interhemispheric communication theory.

Recently, the first biological explanation of the beneficial effect of EMDR was found. De Voogd and colleagues (2018) found evidence that eye movements may reduce amygdala activity, and that large amygdala deactivation was related to a stronger reduction in negative emotions. This deactivation could potentially be a biological explanation of the observed effect of EMDR.

The working memory (WM) account (Andrade, Kavanagh, \& Baddeley, 1997; Gunter \& Bodner, 2008; van den Hout \& Engelhard, 2012) may be a more articulated explanation for the effectiveness of EMDR. The WM account stipulates that retrieving a trauma memory while simultaneously performing eye movements can be seen as a dual task that taxes WM. 


\section{Chapter 1}

The capacity of the WM is limited (Baddeley, 1998) and engaging in two tasks may easily tax WM (Andrade et al., 1997). The consequence of dual task performance is that it interferes with the full-blown retrieval of negative memories. Thus, according to the WM account, the combination of memory retrieval and simultaneously performing eye movements will lead to degraded representations - in terms of vividness and emotionality - of trauma memories. This is also known as imagination deflation (van den Hout \& Engelhard, 2012). As a result, the traumatic memory will be less outstanding (i.e., less emotional) and these degraded memory representations are subsequently stored during reconsolidation. The effect is also observed for positive memories (i.e., positive memories become less positive; Engelhard, van Uijen, \& Van den Hout, 2010; Hornsveld et al., 2011; van den Hout, Muris, Salemink, \& Kindt, 2001). On top of that, imagination inflation was also found for other dual tasks that tax WM. More specifically, complex spatial tapping (Andrade et al., 1997), vertical eye movements (Gunter \& Bodner, 2008), drawing (Gunter \& Bodner, 2008), attentional breathing (van den Hout, Engelhard, Beetsma et al., 2011), playing Tetris (Engelhard, van Uijen, \& van den Hout, 2010), and subtraction (Engelhard, van den Hout, \& Smeets, 2011; van den Hout et al., 2010) all seem to produce comparable effects.

Van den Hout and Engelhard (2012) proposed an inverted U-shape concerning the WM taxation (see also Gunter \& Bodner, 2008). That is, too little or too much taxing will have no or little effect on the vividness and emotionality of memories. Engelhard, van den Hout, and Smeets (2011) found that taxing of the WM increased when a dual task (i.e., subtracting while responding to a reaction time task) becomes more complex (subtract in steps of 1,2 , and 7). When this arithmetic task was performed while recalling a negative memory, they found an inverted U-shape pattern. More specifically, emotionality did not decrease after recall-only or recall and complex arithmetic exercises (i.e., subtract in steps of 7), but it did decrease after recall and slightly complex arithmetic exercises (i.e., subtract in steps of 2). No such pattern was observed for vividness. They concluded that not taxing the WM or taxing it too heavily does not change the quality of the memory, thereby providing evidence for the inverted U-curve. Van Veen and colleagues (2015) examined whether the speed of eye movements would support the inverted U-curve hypothesis. In their second study, participants were asked to retrieve negative memories while performing no, slow $(0.8 \mathrm{~Hz})$ or fast $(1.2 \mathrm{~Hz})$ eye movements. Contrary to the inverted U-curve hypothesis, faster eye movements led to stronger decreases in vividness and emotionality (see also van Schie, van Veen, Engelhard, Klugkist, \& van den Hout, 2016). Littel and van Schie (2019), also, found no evidence for the 
proposed inverted U-curve. Their participants recalled negative autobiographical memories while simultaneously performing either complex (subtract in steps of 7), intermediate (subtract in steps of 2), simple (subtract in steps of 1) or no counting. More decreases in vividness and emotionality were observed in counting conditions with higher WM taxation.

Van Veen, van Schie, van de Schoot, van den Hout, and Engelhard (2020) were the first to show that the effect of eye movements only lead to memory effects in the short-term. Participants recalled a negative autobiographical memory and were asked to rate the memory on vividness, emotionality, and distress before and after the intervention (or recall only) and 24 hrs later. They found the general obtained pattern, that is, the immediate decrease in vividness and emotionality, as well as reductions in distress. However, 24 hours later, the eye movement condition relapsed, while the decreases in the recall-only condition (i.e., control condition) persevered.

It is unclear how changes in vividness and emotionality relate to reductions in PTSD symptoms (Gunter \& Bodner, 2009). In a randomized clinical trial (RCT; Sack et al., 2016), PTSD patients were treated with EMDR and randomly assigned to an eye movement, eye fixating or no explicit visual condition (i.e., control condition). Symptom changes were measured with the clinician administered PTSD scale (CAPS; Blake et al., 1990). No statistically significant differences between eye movements versus control condition (Cohen's $d=2.06$ ) and the eye fixating condition versus control condition (Cohen's $d=2.58$ ) on differences in PTSD symptomatology were observed. It may be possible that changes in vividness and emotionality are not causally linked to changes in PTSD symptomatology (e.g., intrusions; see van Schie, van Veen, \& Hagenaars, 2019). Sack et al. (2016) also concluded that eye movements and eyes fixating must be equally taxing WM. Van Veen, Kang, and van Schie (2019) examined this and found, by using a reaction time task, that performing eye movements taxes WM more strongly than keeping one's eyes fixed. As can be seen, the findings on the relationship between eye movements (or control) and working memory capacity are inconclusive.

Though the working mechanisms might not be completely understood yet, EMDR is now listed as a treatment of choice for PTSD by the National Institute for Health and Clinical Excellence and the World Health Organization (Landin-Romero, Moreno-Alcazar, Pagani, \& Amann, 2018). Even more so, EMDR is used to treat a range of other disorders (e.g., eating disorders, addiction) as well. The justification to use EMDR for a plethora of other disorders is that traumatic events may play an important role in the formation of these disorders (Bloomgarden \& Calogero, 2008). However, it is unknown whether EMDR is an 


\section{Chapter 1}

effective treatment for these disorders. Hudson, Chase, and Pope (1998) were one of the first to examine the effectiveness of EMDR to treat eating disorders. They advised to use EMDR with caution, because when EMDR is used to treat an eating disorder, it seems to suggest a causal link between the eating disorder and a traumatic experience (Hudson et al., 1998). Recently, Cuijpers, van Veen, Sijbrandij, Yoder, and Cristea (2020) examined the effectiveness of EMDR for multiple other disorders. In their meta-analysis, 77 randomized controlled trials were included, in which the effectiveness of EMDR was compared with a control or alternative treatment for mental health problems (e.g., depression, anxiety). For PTSD treatment, EMDR was found to be superior to control (large Hedges' $g=0.93$ ) and other treatment conditions (small to moderate Hedges' $g=0.33$ ). This finding reiterates the superiority of EMDR in the treatment of PTSD. However, these studies were heterogeneous ( $P^{2}$ $=72 \%$ and $56 \%$, respectively) and displayed a high risk of bias (e.g., concealment of allocation to conditions). When only the studies that had a low risk of bias were included, the superior effect of EMDR disappeared (small Hedges' $g=0.07$ ). For anxiety, EMDR was effective (large Hedges' $g=1.46$ ). For other mental health problems (e.g., obsessive-compulsive disorder, schizophrenia), unfortunately, not enough studies were available to draw firm conclusions. EMDR may be effective for PTSD in the short run, but long-term effects are unclear. Because the quality of the included studies was rather low, and hence no conclusions can be drawn, it is not recommended to use EMDR for other mental health problems (e.g., complex childhood trauma; Chen et al., 2018).

As noted above, in EMDR the traumatic memory is retrieved (while performing eye movements) until the subjective vividness and emotionality have declined. This means that during every retrieval attempt, details of the memory might change and this might lead to memory errors. One such memory error that might arise is the emergence of false memories. Therapists do not settle for legal fact-finding during a therapeutic intervention, but for the treatment effectiveness. Thus, it should not matter whether the memory is true or false to begin with. The patient's subjective experience of the memory is central to the therapeutic session. False memories could even have positive effect, in a sense that they could help the patient process the traumatic experience more effectively. Nevertheless, the question is whether the therapy itself can make patients susceptible to produce false memories.

Divided Attention and False Memories. As said before, simultaneously performing eye movements and retrieving a traumatic memory can be seen as a dual task. Studies on divided attention during retrieval demonstrate that divided attention negatively influences 
memory. Dudukovic, DuBrow, and Wagner (2009) studied the effect of divided attention on memory. Their participants had to study and recognize pictures of objects under full attention or divided attention (e.g., simultaneously listening to words) at both encoding and retrieval. Their findings demonstrate higher correct recall and lower memory commission errors for the full attention condition. This indicates that divided attention during retrieval can be harmful for subsequent recollections.

Research on divided attention during memory retrieval has indeed shown that false memory rates increase (see e.g., Fernandes \& Moscovitch, 2000; Knott \& Dewhurst, 2007a,b; Shah \& Knott, 2018). In such studies, participants viewed word lists and during the retrieval phase, participants in the divided attention condition had to perform a dual task (e.g., a random number generator task). Divided attention resulted in overall increased false memories, specifically for negative material (Shah \& Knott, 2018) and decreased overall memory accuracy (e.g., Lozito \& Mulligan, 2006). If there is one aspect within EMDR in which a patient has to divide attention, it is the eye movement phase.

Eye Movements and False Memory. Individuals with mental health problems may believe that therapy has the answers to solve their issues (Brainerd \& Reyna, 2005) and will rely on the experience of their therapist. Considering the legal consequences (e.g., false accusations of abuse), it is imperative to obtain knowledge whether a certain therapy, in this case EMDR, may elicit false memories.

As stated above, traumatic memory is prone to memory errors due to its reconstructive nature (McNally, 2005). The dual representation theory states that reliance on the sensory system results in flashbacks. Because this system is organized in an associative manner, following AAT, it could be assumed that false memories could be produced during this time. Eye movements, as used in EMDR, might be an additional risk factor that may make persons more prone to create false memories. Eye movements (i.e., reduced vividness and emotionality) typically result in the imagination deflation effect (van den Hout \& Engelhard, 2012), which can mean that the memory of the traumatic event becomes more vague. When memory becomes vague and one has to remember the specific event, it is likely that memory gaps will be filled in based on associations (as per AAT), gist traces (as per FTT) or misinformation (as per discrepancy detection and SMF). As a result, the memory of the traumatic event will be distorted (see Figure 1.2). 


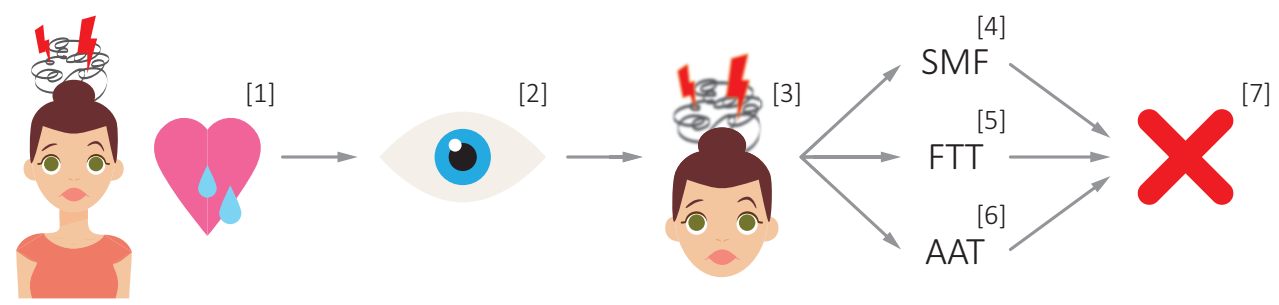

Figure 1.2 Proposed theoretical framework on eye movements as used in EMDR and false memories. [1] An individual witnesses a traumatic event and experiences PTSD symptoms. [2] EMDR is the treatment of choice. [3] Due to the eye movements, the memory of the traumatic event becomes more vague (i.e., less vivid and emotional). Following the false memory frameworks, this can lead to [4] decreased discrepancy between sources of information (i.e., SMF and discrepancy detection); [5] the activation of gist traces (i.e., general information) instead of verbatim traces (i.e., details; FTT); [6] the activation of related, but incorrect nodes in the knowledge network (i.e., AAT). As a result, [7] false details may be recalled.

Literature on potential suggestion-induced and spontaneous false memories effects of eye movements, as used in EMDR, is scarce. Laboratory studies mainly focus on the effect of eye movements, because this part of the EMDR protocol demonstrated the beneficial effect (i.e., reduced vividness and emotionality). For suggestion-induced false memories, Parker, Buckley, and Dagnall (2009) presented pictures to their participants and provided them with misinformation. Afterwards, they were asked to perform horizontal, vertical, or no eye movements. Horizontal eye movements increased true recognition and decreased the misinformation effect. However, this might be due to the performance of eye movements after the misinformation was presented.

Christman, Propper, and Dion (2004; Experiment 2) used the DRM paradigm to examine the effect of eye movements on spontaneous false memories. Participants heard the word lists and were subsequently asked to perform eye movements or not. Hereafter, they performed a free recall task. Eye movements led to fewer false recall, but accuracy during recall did not increase compared with the control condition. Parker and Dagnall (2007) used the DRM paradigm as well, but participants completed a recognition test. Horizontal eye movements increased true memory rates (see also Parker \& Dagnall, 2012; but see Matzke et al., 2015) and decreased false recognition, compared with the vertical eye movement and control condition. Devilly and Brown (2011) also employed word lists and tested their participants' memory by means of a free recall and recognition test. No statistically significant difference in false recall between the eye movement and control condition emerged, but the authors did find that participants in the eye movement condition had higher true and false recognition rates. 
In three experiments, Nieuwenhuis and colleagues (2013) examined the effect of performing horizontal eye movements (Experiment 1), tactile (Experiment 2) or auditory stimulation (Experiment 3) on memory retrieval. Participants viewed Dutch words and were asked to perform eye movements, tapping, or heard clicks. Hereafter, they were asked to complete a free recall test. The eye movement and tactile conditions had higher true and lower false recall rates compared with the control condition.

The abovementioned studies, however, differ in their procedure (e.g., duration of eye movements). More importantly, eye movements were performed after memory recall, while during EMDR, memory recall and eye movements are performed simultaneously. Also, their theoretical framework was the interhemispheric hypothesis, which has been discarded (see above). Thus, these studies do not provide sufficient information on whether eye movements as used in EMDR may promote false memories.

\section{Legal relevance of EMDR}

Psychological interventions target autobiographical memories and the aim is to alter these memories (Phelps \& Hofmann, 2019). Not only is traumatic memory reconstructive (McNally, 2005), the findings described above indicate that memory errors during therapy are likely to occur. As false memories might be beneficial in some circumstances (e.g., memories become more positive or the individual can process the negative experience more efficiently), this can be problematic when such memories enter the legal arena, because it can lead to wrongful prosecutions and convictions.

In the Netherlands, allegations potentially involving recovered or false memory claims are often referred to the Landelijke Expertisegroep Bijzondere Zedenzaken (LEBZ). The main task of the LEBZ is to advise the public prosecutor on whether or not to pursue criminal investigation. The LEBZ has to be consulted when the police report includes aspects of: (a) recovered memories of childhood (sexual) abuse, (b) memories of childhood (sexual) abuse before the age of three, and/or (c) ritual abuse (Nierop \& van den Eshof, 2010). Experts of the LEBZ work independently on the given case file. During a meeting, they will report on their findings based on their own expertise. When one perspective has been put forward, a final report will be sent to the public prosecutor.

The popularity of EMDR is also evident in several LEBZ cases. In the period 20042013, 34 (of the 50) cases included EMDR ${ }^{3}$. In 14 cases, the role of EMDR in the recovery of memories was questionable. More specifically, in some of these cases auditory memories 


\section{Chapter 1}

were present, but no images. In eight cases, no memories of sexual abuse were present at the start of therapy, but surfaced during EMDR therapy. Though these numbers might seem modest, such cases can have serious legal consequences. More specifically, if such cases enter the legal arena, there is a possibility that an innocent defendant will be wrongfully prosecuted. It is important that victims are taken seriously and feel heard by all parties involved (i.e., therapist, lawyer, judge), but it is also important that legal professionals are well aware of the false memory potential in cases involving EMDR, if such potential exists. 


\section{Aims and Outline of Dissertation}

The aim of the current dissertation is to examine whether EMDR increases false memory susceptibility (see Figure 1.3). To examine this potential, the studies presented in this dissertation focused on EMDR therapists' knowledge about the functioning of memory and susceptibility to false memories when performing eye movements used in EMDR. In Section I (Chapters 2 and 3), general aspects of EMDR will be discussed. More specifically, Chapter 2 describes individuals' expectations after receiving metaphoric instructions (e.g., memory works as a video camera or one can have a photographic memory) as used in the Dutch EMDR protocol. Chapter 3 presents the results of a meta-analysis on the effect of eye movements versus alternative dual tasks on the vividness and emotionality of negative autobiographical memories. The label EMDR emphasizes the importance of eye movements. In practice, however, alternative dual tasks are used to obtain the same beneficial effect (i.e., reduced vividness and emotionality). Section II (Chapters 4, 5, and 6) focuses on the link between eye movements, as used in EMDR, and false memories. This section will commence with how knowledgeable EMDR practitioners are about the functioning of memory. More specifically, Chapter 4 presents the results of a survey of EMDR practitioners about their beliefs about the
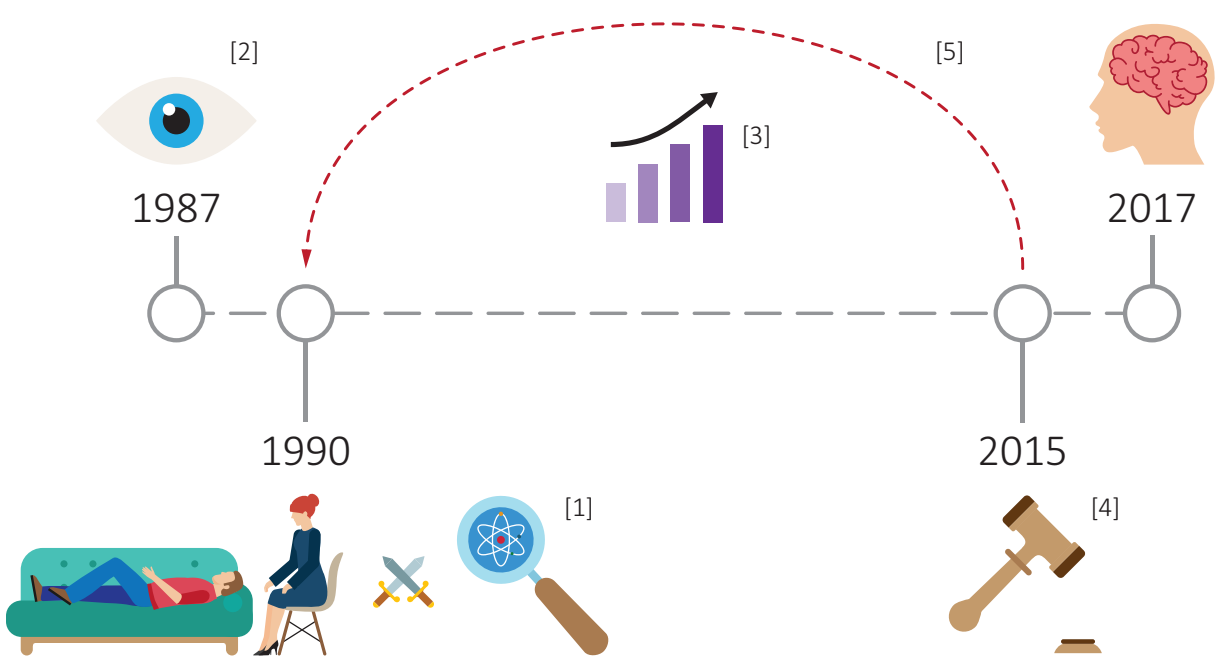

Figure 1.3 Background of research question. [1] Recovered memory debate; [2] Development of EMDR; [3] EMDR increases in popularity for, among others, PTSD treatment; [4] Popularity of EMDR visible in Dutch legal cases, but [5] the sudden occurrence of traumatic memories during/after EMDR reminds expert witnesses of the recovered memory debate; which led to [6] the research question of this dissertation is to examine whether EMDR increases false memory susceptibility. 


\section{Chapter 1}

functioning of memory, including the belief in repressed memories. Chapter 5 presents an experimental study testing the susceptibility to suggestive false memories after performing eye movements, as used in EMDR. In this way, we could experimentally examine what would happen to participants' memory when they are presented with misinformation (e.g., when a therapist would present misinformation). Suppose that a therapist has correct knowledge about the functioning of memory and withholds from presenting misinformation, it could still be the case that the intervention itself could elicit false memories. With this in mind, Chapter 6 presents an experimental study that examined the vulnerability to producing spontaneous false memories after performing eye movements as used in EMDR. The dissertation ends with a discussion of the most important findings in this research and their practical implications (Chapter 7).

\section{SECTION I}

Chapter 2. To identify the target image, Dutch EMDR practitioners can choose between two metaphorical instructions: video or photobook metaphor. However, it is unknown what type of expectations are elicited with such instructions. In this study, 160 participants were randomly assigned to either one of the two metaphorical instructions or a control instruction and were asked to indicate how vivid and emotional they expect to recall the traumatic memory, how credible they found the instruction, and how reliable they would evaluate a therapist providing such an instruction.

Chapter 3. Though eye movements are a central component of EMDR, in practice, alternative dual tasks are employed during EMDR therapy (e.g., finger tapping). This chapter presents the results of a meta-analysis on the effect of eye movements versus alternative dual tasks on the vividness and emotionality of negative autobiographical memories. Fifteen experimental studies were included in the meta-analysis.

\section{SECTION II}

Chapter 4. In this study, we examined EMDR practitioners' beliefs about memory. In two survey experiments, we examined their beliefs in correct and controversial statements about the functioning of memory. In Experiment 1, 35 mental health students and 12 EMDR practitioners rated the authenticity of a fictitious case vignette and provided their opinion on 
15 memory statements (Ost et al., 2013, Patihis et al., 2014). In Experiment 2, 24 students, 41 EMDR practitioners, and 35 academics provided their opinion on 18 memory statements (Magnussen \& Melinder, 2012; Ost et al., 2013; Odinot, Boon, \& Wolters, 2015). This survey aimed to examine how knowledgeable EMDR practitioners are about the functioning of memory. Questionable beliefs about memory could lead to, unintentionally, suggestive questioning during a therapeutic session. This is the research question of the subsequent chapter.

Chapter 5. What would happen if suggestive information would be presented to a patient who is treated with EMDR? In this chapter, the susceptibility to endorse misinformation in one's own memory report is examined. Eighty-two participants saw a video depicting a car crash. Half of them performed eye movements or held their eyes stationary. Afterwards, all participants received misinformation by means of an eyewitness narrative. A recognition test was completed to examine the number of correct memories and endorsed misinformation items. However, even when there is no misinformation presented to the patient, it is still possible that the intervention itself can make individuals prone to create false memories. This will be examined in the next chapter.

Chapter 6. If an EMDR therapist correctly follows the EMDR protocol and withholds from suggestive information/questioning, it might, in principle, still be possible for a patient to produce false memories spontaneously. The study in this chapter examined the vulnerability to spontaneous false memories after performing eye movements. In Experiment 1, 72 participants viewed DRM word lists containing negative and neutral associated words. They were given a free recall and recognition test immediately after this. In Experiment 2, 68 participants followed the same procedure, but completed both memory tests 48 hours later. During the free recall, half of the participants were instructed to perform eye movements. True and false memory rates were measured during the free recall and recognition test. 

SECTION I 


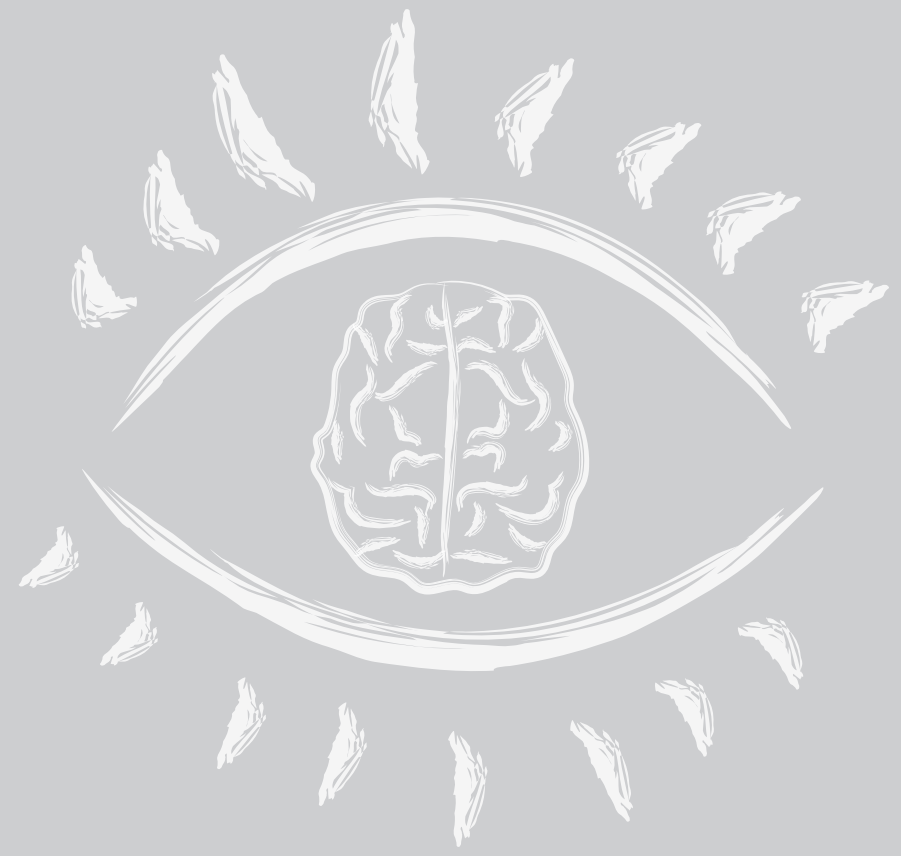




\section{CHAPTER 2}

\section{Metaphorical instructions in Eye Movement Desensitization and Reprocessing}

Published as:

Houben, S. T. L., Otgaar, H., \& Roelofs, J. (in press). Psychological myths as therapeutic instructions in Eye Movement Desensitization and Reprocessing. The Journal of Psychology: Interdisciplinary and Applied. 



\section{INTRODUCTION}

People only use $10 \%$ of their brain, the polygraph is an accurate measure for deception detection, and hypnosis is useful for retrieving memories. These are only a few examples of psychological myths that are endorsed by non-trivial percentages of the general population (see e.g., Lilienfeld, Lynn, Ruscio, \& Beyerstein, 2010; Taylor \& Kowalski, 2004). Although endorsing such myths might not be harmful for laypersons, it can be perilous if certain professionals hold such beliefs. For example, a therapist might adopt the treatment plan in line with a controversial belief (e.g., repressed memory), which may be harmful for the patient. Therapists might believe in the existence of repressed memories and might actively search for the existence of such repressed memories, thereby increasing the likelihood of inducing false memories (i.e., memories of an event that did not happen; Loftus, 1993; Otgaar, Howe, Patihis, et al., 2019).

In the beginning of the 1990s, a heated debate on one specific psychological myth (i.e., the existence of repressed memories) dominated the psychological field (Crews, 1995). Researchers and clinicians argued about the accuracy of childhood sexual abuse memories that surfaced during psychotherapy and did not exist before therapy (i.e., recovered memories). Clinicians argued that individuals unconsciously block traumatic autobiographical experiences, making them inaccessible for retrieval (i.e., repressed memory). Furthermore, they argued that psychotherapy could unblock these memories and recover them (Ceci \& Loftus, 1994). Researchers argued that the existence of repressed memories has not been supported by any empirical evidence. More specifically, one important argument against repressed memories was that a wealth of research has shown that traumatic memories are well retained and accessible (e.g., McNally, 2003). Furthermore, memory scholars argued that the emergence of these memories during therapy could be the result of suggestive therapeutic instructions potentially leading to the formation of false memories (Loftus \& Pickrell, 1995).

To understand whether the belief in repressed memories is a widespread phenomenon, several survey studies examined memory beliefs in different groups of professionals, such as clinicians. An early study by Yapko (1994) found that around 60\% ( $n=516)$ of therapists believed in the existence of repressed memories. In a recent overview, it was found that when the majority of survey studies are combined, $70 \%(n=2305)$ of clinicians indicated to believe in the existence of repressed memories (Otgaar, Howe, Patihis, et al., 2019). More 


\section{Chapter 2}

interestingly, in the 1990s this percentage was 61\% and increased to 76\% from 2010 onwards. It thus seems that psychological myths are still widely believed among clinicians. In the current study, we were not interested in this pervasive belief, but are focused on the incorrect belief that our memory records everything we experience, just like a video recorder (Lilienfeld et al., 2010). The question that we raised was what would happen if such a psychological myth were embedded in a therapeutic instruction.

This question is of interest because the Dutch protocol for Eye Movement Desensitization and Reprocessing (EMDR, an intervention to treat post-traumatic stress disorder; Shapiro, 1989) includes two psychological myths as an instruction to select a target image (so called hot spot), which will become the focus point during therapy. The selection of the target image differs from the original EMDR protocol (Hornsveld et al., 2017a,b,c; Hornsveld et al., 2018a,b). More specifically, to select the target image, in the Dutch protocol, a therapist can choose between two metaphorical instructions, which are known to be psychological myths. A therapist can select either a video metaphoric instruction: "You've just told me how this memory is stored in your mind. Now I am asking you, what presently is the most disturbing image of this memory? Play the movie of the memory, pause it, when you see the most disturbing image. We are searching particularly for an image with you in it. It should not be the image of what you found most disturbing at that time, but what is now, at this moment, the most disturbing image to look at, including images that show what could have happened, or images that become a part of the movie later on."(..) "What does this image look like?" or a photobook instruction: "Imagine you have a photo album in your head, with photos or snapshots that show how you remember this event/incident momentarily; it's possible that you see images of things that did not really happen, but were added to the album later on. We are looking particularly for an image with you in it. Which photo is at this moment the worst to look at?(...)"What do you see on the photo?" (de Jongh \& ten Broeke, 2016, p. 148, translated by Giuditta Soro in 2013, adapted by Steven Meijer in 2016).

The reason why these metaphoric instructions can be problematic, is that patients might incorrectly assume that memory works as a video or photo album. Furthermore, they might reason that when they retrieve a memory, it should be highly vivid and detailed just as a video or photo. The consequence could be that they spontaneously add details to their account, which might be false, but give the allure of a vivid and detailed memory (see e.g., Houben, Otgaar, Roelofs, Smeets, \& Merckelbach, 2020). Although recent survey studies have shown that individuals do not endorse former popular psychological myths (e.g., 
"memory works as a video camera, accurately recording everything that is experienced" or "people can have a photographic memory"; Brewin, Li, Ntarantana, Unsworth, \& McNeilis, 2019; Houben et al., 2019), it is intriguing that such psychological myths are included in a therapeutic instruction. Although the Dutch EMDR protocol is revised each year based on clinical experiences of therapists and scientific results (Hornsveld et al., 2017a), these metaphoric instructions are still included in the protocol.

The aim of this study was to explore how participants would evaluate such an instruction (i.e., on expected vividness and emotionality, therapeutic instruction credibility, and reliability of the therapist). Furthermore, we were interested if such an instruction influences participants' beliefs about memory (e.g., the belief in repressed memory). To achieve this aim, participants received either the video, photo, or a control instruction and were asked to evaluate the instruction accordingly. Next, participants completed a memory survey. We expected that the instructions would elicit high ratings of expected memory vividness and emotionality. In addition, we expected that the video and photobook instruction would heighten the belief in the statements "memory is like a computer/video camera, accurately recording events as they actually occurred" and "some people have true photographic memories", respectively.

\section{METHOD}

\section{Participants}

We recruited 160 undergraduate students $\left(\right.$ Mean $_{\text {age }}=20.18$, Standard Deviation $=1.88$, range 17 - 30, 129 women) from a Bachelor's psychology program. Participants with mental health issues (e.g., anxiety or depression) were advised to refrain from participation. All participants provided consent and received course credits for their participation. The standing ethical committee for the Faculty of Psychology and Neuroscience approved the study. A sensitivity analysis showed that with the current sample size and design, a medium effect size could be detected ( $f=0.25$; Faul, Erdfelder, Lang, \& Buchner, 2007).

\section{Materials and Procedure}

All data and materials are available at the Open Science Framework (https://osf.io/jkv5w). The survey was in English and took about ten minutes to complete and was completed online by using Qualtrics (Provo, Utah, USA) software. Participants received the link to the 


\section{Chapter 2}

questionnaire and completed it at their own pace. Participants were debriefed once the questionnaire was completed.

Metaphoric Instruction. Participants had to imagine being a patient with longstanding emotional problems and in need for therapy. Then they randomly received one of the three therapeutic instructions (video instruction $n=53$, photo instruction $n=50$, control instruction $n=57$; see https://osf.io/r4xeb) and were asked how they would select the video, photo, or memory, respectively. There was no word limit for this answer. The video and photobook instruction were derived from de Jongh and ten Broeke (2016). The control instruction was based on these instructions as well, but without any mentioning of a video or photobook. Instead, the control condition reiterated that human memory does not store every experience and that forgetting is normal. All instructions were equally long to prevent differences based on length (video: 100 words; photo: 102 words; control: 112 words). Participants who received the video instruction needed, on average, 4.5 minutes to report their memory, participants who received the photo instruction 6 minutes on average, and the control participants needed, on average, 5 minutes to answer how they would select the memory.

Thereafter, participants answered four questions pertaining to the therapeutic instruction they received. Participants indicated how vivid and emotional they thought they would need to recall the trauma image on a visual analogue scale ( $0=$ not very vivid) emotional; 100 = very vivid/emotional), how credible they found the therapeutic instruction ( 0 = not very credible; 100 = very credible) and how reliable they would consider a therapist providing this therapeutic instruction ( $0=$ not very reliable; $100=$ very reliable). They also had the option to elaborate on the chosen score.

Statements about Memory. The questionnaire (see https://osf.io/j7rth) consisted of 15 memory-related statements (Houben et al., 2019; Ost et al., 2013; Patihis et al., 2014). Two correct statements (e.g., "Memory can be inaccurate") and 13 controversial statements (e.g., "repressed memories of event that did happen can be retrieved in therapy accurately"; "even very vivid memories can be false"; "the body may remember trauma outside of the mind's awareness") were included. Participants indicated their opinion ( 1 = agree, 2 = disagree, 3 = no opinion). 


\section{RESULTS}

Metaphoric Instruction. Participants in the video condition who elaborated on how they chose the video indicated they would recall a disturbing memory $(n=29)$ and subsequently play it as a movie in their mind $(n=29)$. A minority $(n=4)$ found the instruction confusing. Participants in the photo condition would choose a memory that was disturbing $(n=37)$, and specifically aimed to recall an actual picture $(n=11)$. Control participants $(n=26)$ indicated they would select a memory that was most disturbing at this point.

Mean VAS scores can be found in Table $\mathbf{2 . 1}$ and the respective explanations can be found on https://osf.io/dkasc. A one-way ANOVA was performed on vividness, emotionality, credibility, and reliability. For vividness, a statistically significant effect of condition emerged $\left(F(2,157)=5.05, p=.008, \eta_{\text {partial }}^{2}=.06\right)$. A Bonferroni post hoc test revealed that participants in the video condition (Mean $=71.30$, Standard Deviation $=16.62$ ) opined to recall the trauma more vividly than the photo condition (Mean $=60.80$, Standard Deviation $=21.89$; $p=.020)$. Participants in the video condition (Mean $=71.30$, Standard Deviation $=16.62)$ were also more likely to opine to recall the trauma more vividly than the control condition $($ Mean $=61.12$, Standard Deviation $=19.39 ; p=.020)$. A majority of participants in all three conditions who reported high vividness scores (i.e., VAS scores $>60$ ) indicated that a vivid memory is necessary to overcome the trauma (i.e., that it is necessary for therapy to report many details; video: $n=17$; photo: $n=11$; control: $n=10$ ). As can be seen from Table 2.1, participants in the video condition were inclined to recall the memory more vividly. Photo and control participants who reported low vividness scores (i.e., VAS scores $<40$ ) reasoned that the memory might be vague or contain false details and as a result they would not report a vivid memory (photo: $n=5$; control: $n=6$ ).

Table 2.1 Mean Ratings and Standard Deviations (in parentheses) for Vividness, Emotionality, Credibility and Reliability per Condition.

\begin{tabular}{lllc}
\hline & Video $(\boldsymbol{n}=\mathbf{5 3})$ & Photo $(\boldsymbol{n}=\mathbf{5 0})$ & Control $(\boldsymbol{n}=\mathbf{5 7})$ \\
\hline How vivid do you think you need to recall the trauma? & $71.30(16.62)$ & $60.80(21.89)$ & $61.12(19.38)$ \\
How emotional do you think you need to recall the trauma? & $66.32(17.64)$ & $60.32(23.56)$ & $65.00(22.05)$ \\
How credible do you find the therapeutic instruction? & $53.58(23.10)$ & $49.72(24.73)$ & $55.95(23.12)$ \\
How reliable would you find a therapist providing this thera- & $55.91(23.20)$ & $51.40(24.72)$ & $54.84(22.06)$ \\
peutic instruction? & & &
\end{tabular}

Note. Answer scales range from 0 (not vivid/emotional/credible/reliable) to 100 (very vivid/emotional/credible/ reliable). ${ }^{*}=p<.05$. 
There was no statistically significant difference between the photo and control condition ( $p=1.00$ ). For emotionality, credibility and reliability, no statistically significant effect of condition emerged (all p's > .275). For emotionality, participants who reported high VAS emotionality scores mentioned that emotional memories would be beneficial for therapy (video: $n=9$; photo: $n=6$; control: $n=6$ ), but that it is also a normal reaction when recalling a traumatic event (video: $n=3$; photo: $n=3$; control: $n=4$ ). Participants who provided low VAS scores reasoned that it is important to prevent that emotions take over or that a patient starts reliving the trauma (video: $n=2$; photo: $n=3$; control: $n=0$ ). As for credibility, participants who provided high credibility scores indicated that the instructions seem professional and that it would be necessary to trigger the origin of the trauma (video: $n=9$; photo: $n=8$; control: $n=18$ ). Participants who provided low VAS scores said the instructions were too long, vague, and confusing (video: $n=6$; photo: $n=13$; control: $n=6$ ). For reliability, participants who provided high scores indicated that therapists itself are reliable (video: $n=10$; photo: $n$ $=5$; control: $n=8$ ). Participants who provided low reliability scores stated that because of the unclear instructions, it seemed that the therapist does not know what (s)he is doing (video: $n=4$; photo: $n=4$; control: $n=3$ ).

Statements about Memory. Table 2.2 gives an overview of all scores and effect sizes. Here, we will highlight some of the most important results reported in Table $\mathbf{2 . 2}$. Participants agreed with the statement that vivid memories can be false (video: $n=50$, 94.3\%, 95\% Cl [83.4-98.5], photo: $n=47,94.0 \%, 95 \% \mathrm{Cl}$ [82.5-98.4], control: $n=54,94.7 \%$, $95 \% \mathrm{Cl}[84.5-98.6])$. A minority of the video $(n=10,18.9 \%, 95 \% \mathrm{Cl}$ [9.9-32.4]), photo ( $n=16$, $32.0 \%, 95 \% \mathrm{Cl}[19.9-46.8])$ and control condition ( $n=12,21.0 \%, 95 \% \mathrm{Cl}[11.8-34.3])$ agreed with the statement that the more intense the emotion in response to a memory is, the more likely the memory is to be accurate. A majority of the video and control condition $(n=40$, 75.5\%, 95\% Cl [61.4-85.8] and $n=37,64.9 \%, 95 \% \mathrm{Cl}$ [51.1-76.8], respectively) agreed with the statement that repressed memories can be retrieved in therapy accurately. Only about half of the participants in the photo condition ( $n=28,56.0 \%, 95 \% \mathrm{Cl}$ [41.1-69.7]) agreed with this statement. More than half of the participants agreed with the statement that some people have true photographic memories (video: $n=31,58.5 \%, 95 \% \mathrm{Cl}$ [44.2-71.6], photo: $n=31$, 62.0\%, 95\% Cl [47.1-75.0], control: $n=35,61.4 \%, 95 \% \mathrm{Cl}$ [47.6-73.7]). Only a few participants $(n=2,3.8 \%, 95 \% \mathrm{Cl}[0.6-14.7], n=2,4.0 \%, 95 \% \mathrm{Cl}[0.7-14.9]$, and $n=3,5.3 \%, 95 \% \mathrm{Cl}[1.4-$ 15.5], respectively) agreed with the statement that memory is like a computer/video camera, accurately recording events as they actually occurred. 


\section{DISCUSSION}

This study explored how participants would evaluate metaphoric instructions used in the Dutch EMDR protocol, which are psychological myths concerning the functioning of memory (Lilienfeld et al., 2010). Furthermore, participants completed a memory beliefs survey to examine whether such an instruction would influence their beliefs on how memory operates. Our results showed that all instructions led participants to expect to recall an emotional memory. In addition, we found that the use of the video instruction yielded an expectation that the recalled memory must be highly vivid. Though a vivid memory is beneficial to work on during the EMDR therapy, this expectancy effect could lead to certain therapeutic demand characteristics (Kanter, Kohlenberg, \& Loftus, 2002). For example, as was evident from participants' elaborations, patients might believe they should report as many details as possible, irrespective if they were authentic. Littel, van Schie, and van den Hout (2017) claimed that (in)correct knowledge about EMDR's working mechanism (i.e., performing eye movements and simultaneously recalling the target image) does not contributes to its effectiveness. However, the current study shows that the instruction to select the target image does elicit a treatment expectancy effect during this phase of the treatment protocol.

Interestingly, the credibility of the therapeutic instruction and the reliability of the therapist providing such an instruction were at chance level (i.e., around 50\%). For example, participants expressed their trust in the capability of a therapist, but the confusing instructions were a reason to question the therapist's reliability. This is an important finding, as treatment credibility and therapist reliability predict the therapeutic alliance (Fjermestad et al., 2017). A strong therapeutic alliance is associated with patient attendance (Shirk, Karver, \& Brown, 2011) and it increases the chance of a positive therapy outcome (Owen, Idigo, \& Valentine, 2015). Hence, the use of such a metaphoric instruction during EMDR therapy might jeopardize the therapeutic alliance.

In line with previous research (Brewin et al., 2019; Houben et al., 2019), only a minority endorsed the controversial belief that memory works as a video camera, accurately recording events as they occurred. Half of all the participants endorsed the belief that some individuals have a photographic memory. Interestingly, a majority of participants (around 95\%; see Table 2.2), irrespective of therapeutic instruction, believed in the existence of repression. This finding is in line with the prevalence of belief in repression (see Otgaar, Howe, Patihis, et al., 2019). 


\section{Chapter 2}

EMDR aims to decrease the vividness and emotionality of negative autobiographical memories. To elicit a target image to work with during therapy, therapists are able to choose between a video or photo instruction. Participants who received the video instruction expected to recall more vivid memories than participants who received the photo instruction. However, neither the instruction itself nor the therapist providing such an instruction were perceived as highly credible and reliable.

Although these findings provide some insight on how the use of metaphorical instructions could be evaluated by patients, future research is needed to draw firm conclusions. For example, a study is needed to explore how actual patients evaluate such an instruction. A limitation of the current study was that a Bachelor's psychology student sample was included. Though psychological myths concerning, for example, the functioning of memory are not explicitly covered in the Bachelor's educational program, some students might have pre-existing knowledge about psychological myths, which was not checked for in the current study. Furthermore, participants could earn extra credits by participating in this study, which could be a confounding factor. Another limitation is that the study was completed online. Therefore, participants who needed psychological support during their imagination could not ask questions directly, but were able to refer for help and assistance afterwards. More importantly, it is still unclear how a therapeutic instruction can influence memory. Although the instructions did not influence memory beliefs per se, it could still influence the content of the memory that is being recalled (e.g., more and/or incorrect details are reported). However, a reliability analysis indicated a poor reliability (Cronbach's $\alpha=0.57$ ) of the memory questionnaire. Future research should include an improved questionnaire to obtain more reliable memory beliefs.

Though the instructions do elicit vivid and emotional memories, it may be a threat to the therapeutic relationship. During the next yearly revision of the Dutch EMDR protocol, it might be important to examine whether the instruction could be altered in a way that (1) it still elicits vivid (and emotional) memories and (2) is perceived as credible so the therapeutic alliance is not at risk. For example, a therapist could instruct a patient to select a memory that is most disturbing to remember at this moment in time, and ask what the patient hears/ sees/feels/smells/etc. Additionally, the therapist should educate the patient that memory is reconstructive and details might be forgotten or added later on.

To conclude, in the current study we examined how participants would evaluate a metaphorical instruction. An expectancy effect was found for vividness, implying that instructions 
containing psychological myths might be detrimental for the therapeutic relationship. As the therapeutic relationship is important for therapeutic success, more research is needed on the effect of such instructions.

Once a target image (and a positive and negative cognition) has been identified, a therapist can proceed to the desensitization phase. In this phase, a patient is asked to retrieve the target image and simultaneously perform eye movements. In practice, however, patients also perform alternative dual tasks (e.g., bilateral tones), as they prefer them more than eye movements (van den Hout et al., 2012). Despite its use in clinical practice, it is unknown whether such alternative tasks have similar effects on the vividness and emotionality of a traumatic memory as eye movements do. In the next chapter, I will present a meta-analysis that compared the effectiveness of both tasks on vividness and emotionality decreases. 


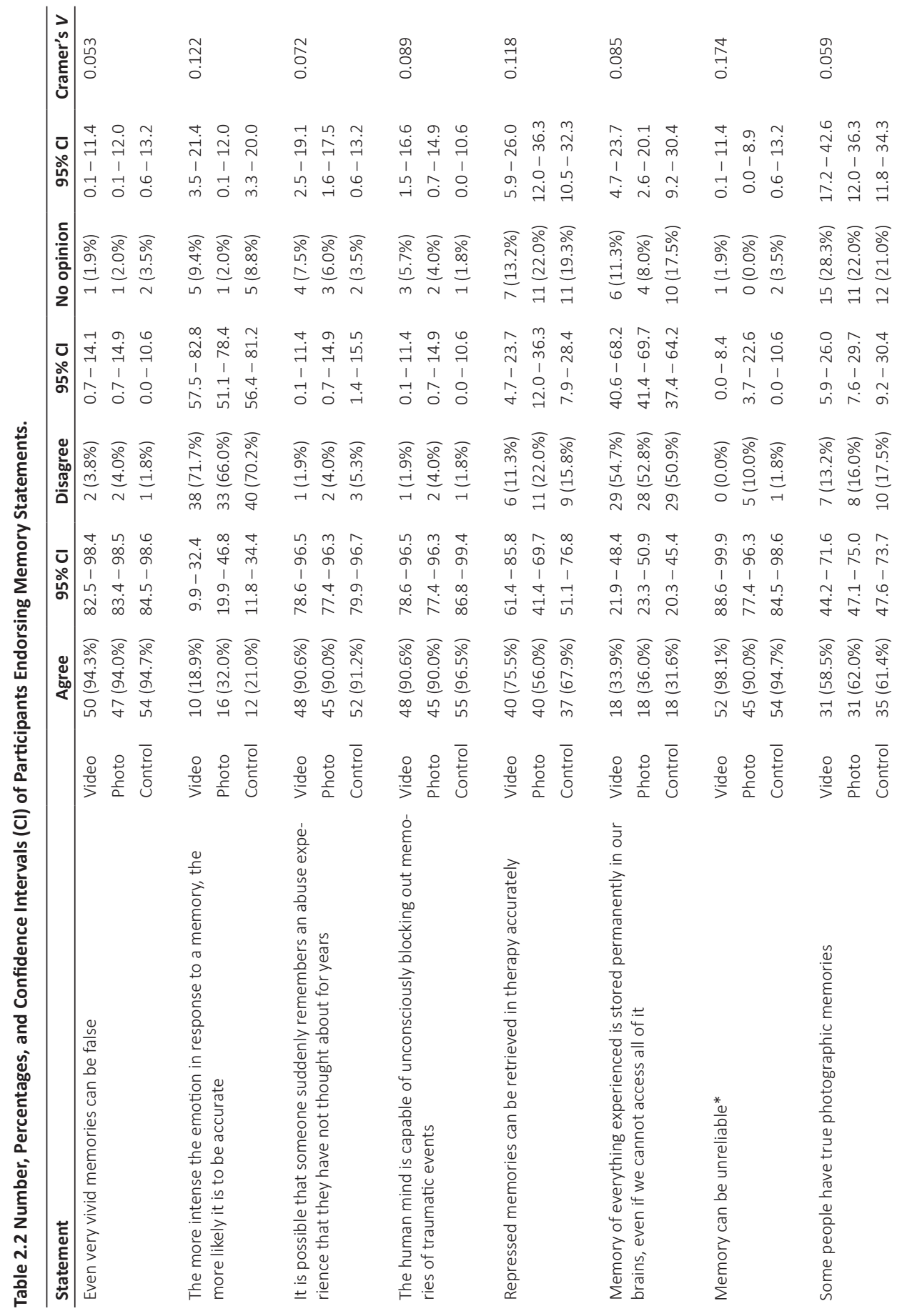




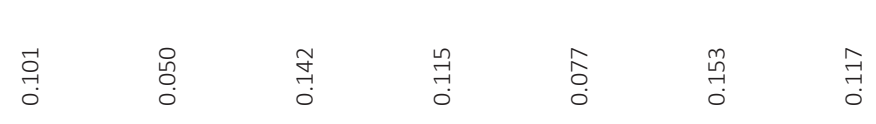

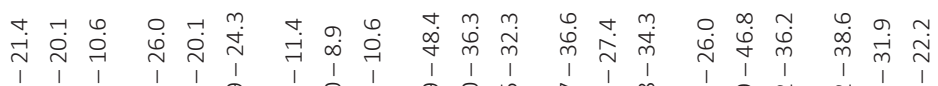

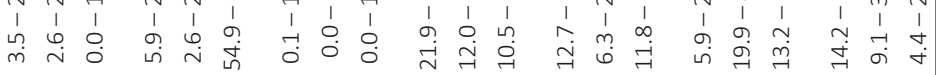

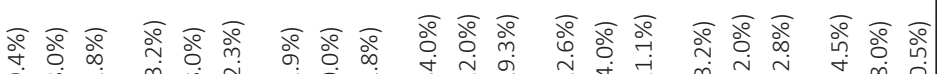

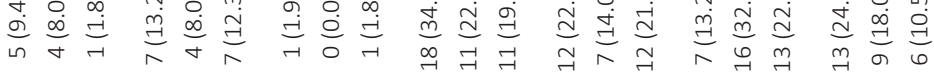

ન゙

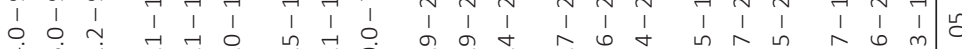

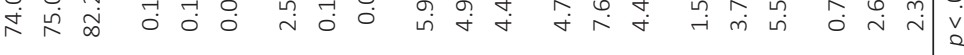

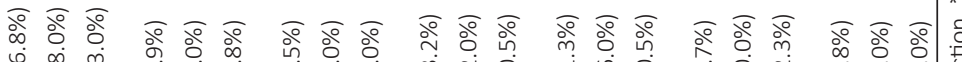

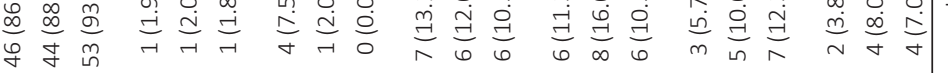

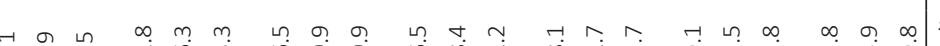

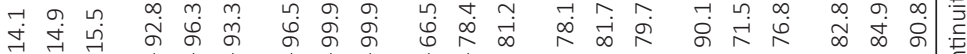

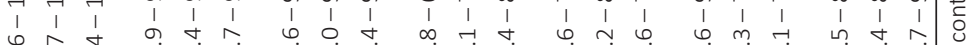

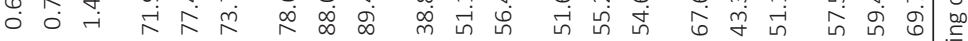

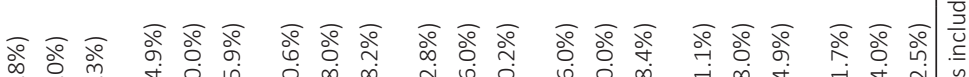
ஸ்

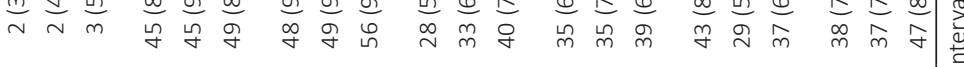

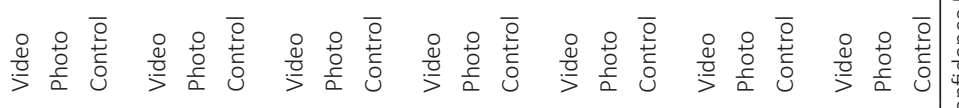

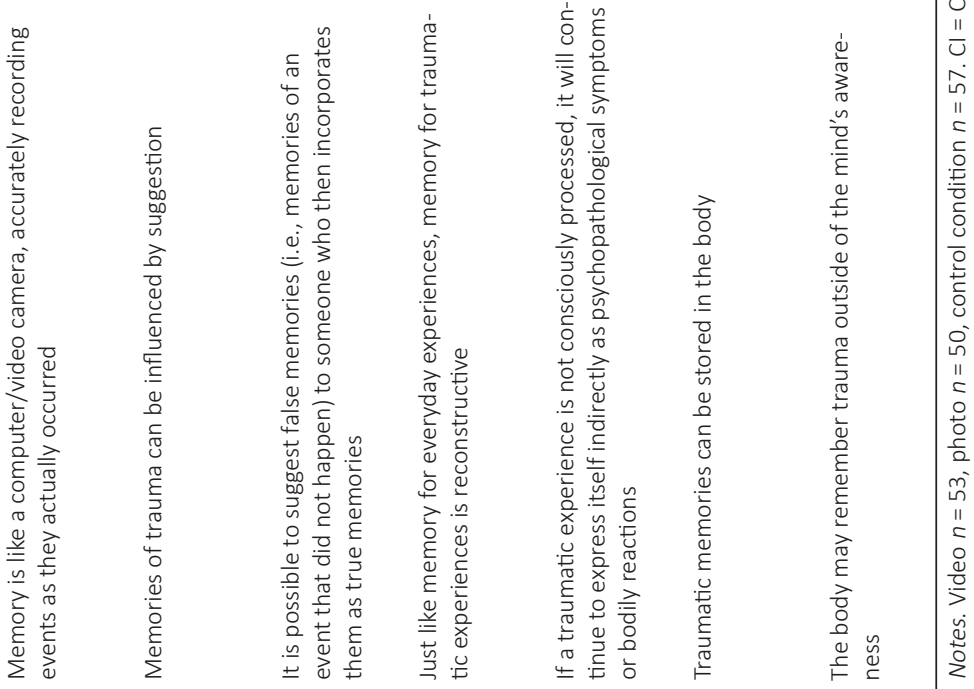




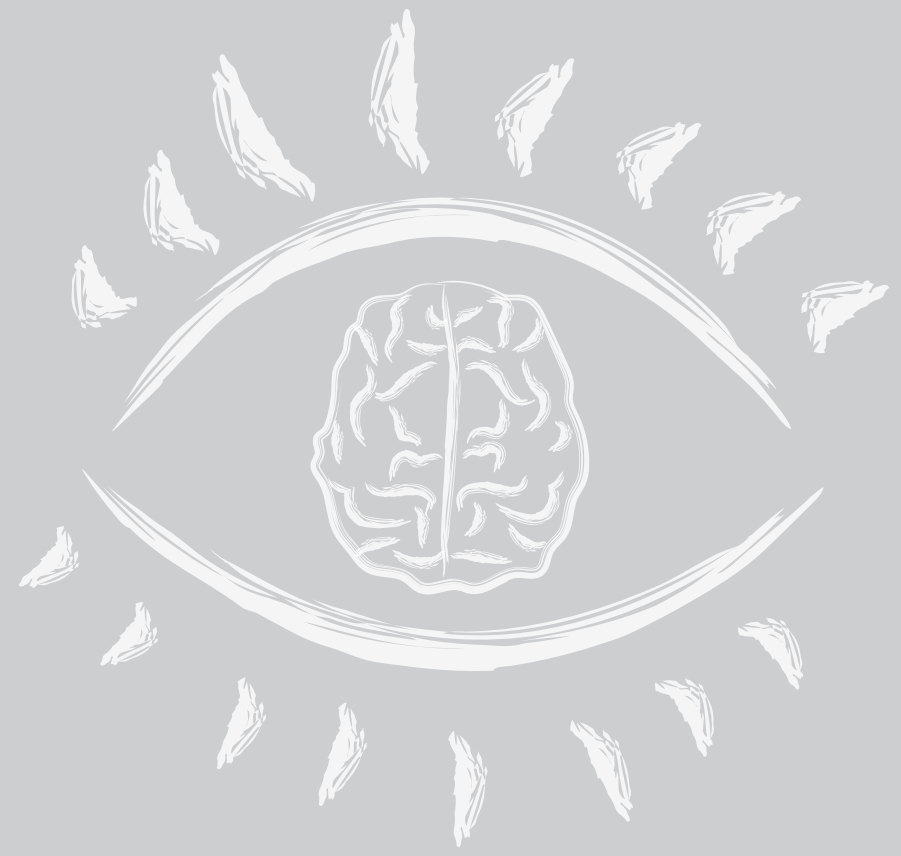




\section{CHAPTER 3}

The effects of eye movements and alternative dual tasks on the vividness and emotionality of autobiographical memory: A meta-analysis of laboratory studies

Published as:

Houben, S. T. L., Otgaar, H., Roelofs, J., Merckelbach, H., \& Muris, P. (2020). The effects of eye movements and alternative dual tasks on the vividness and emotionality of negative autobiographical memories: A meta-analysis of laboratory studies. Journal of Experimental Psychopathology. https://doi.org/10.1177/2043808720907744 



\section{INTRODUCTION}

Patients with post-traumatic stress disorder (PTSD) suffer from memory-related symptoms such as intrusions (i.e., recurrent, involuntary, and distressing recollections of the traumatic event) and flashbacks (i.e., dissociative reactions in which the individual feels as if the traumatic event is recurring) after exposure to a trauma (American Psychiatric Association, 2013). Over the past decades, Eye Movement Desensitization and Reprocessing (EMDR; Shapiro, 1989) has become increasingly popular as a treatment method for PTSD.

During EMDR therapy, the patient is instructed to retrieve and visualize a disturbing memory of the traumatic experience, and to focus on this memory and its related thoughts and feelings while simultaneously performing eye movements (Shapiro, 2002). A commonly voiced view is that eye movements make the negative emotions attached to the trauma memory less outstanding by reducing their vividness and emotionality.

Experimental studies examining the mechanisms of action underlying EMDR have mainly focused on the eye movement component of EMDR (see, for a review, LandinRomero et al., 2018). The typical procedure in such studies is that participants are instructed to retrieve emotional autobiographical memories and to rate these memories on vividness and emotionality (pre-test). Next, some participants have to retrieve the memory whilst simultaneously performing eye movements, whereas other participants recall the memory without performing eye movements (i.e., control condition). Afterwards, participants again rate their memories on vividness and emotionality (post-test). Lee and Cuijpers (2013) conducted a meta-analysis on how eye movements affected emotional memories, notably their vividness and emotionality. Fifteen clinical trials and 11 laboratory studies that compared standard EMDR procedures with eye movements against EMDR procedures without eye movements were included. The clinical studies relied on the Subjective Units of Distress scale (SUDs) and Validity of Cognition (VoC) as outcome variables. The SUDs measures the degree of distress elicited by the trauma memory, while the VoC assesses the believability of a positive cognition in relation to that memory. The laboratory studies relied on Likert-type vividness and emotionality scales.

Overall, Lee and Cuijpers (2013) found that the SUDs declined more in participants who received EMDR with eye movements than in those who were given EMDR without eye movements. This between-treatment difference was associated with a medium effect size (Cohen's $d=0.53$ ). EMDR with eye movements also yielded greater increases in the VoC 


\section{Chapter 3}

than EMDR conducted without eye movements, a difference that was associated with a large effect size: Cohen's $d=0.72$. In a similar vein, the laboratory studies observed larger reductions in vividness and emotionality ratings of negative autobiographical memories following an EMDR procedure with eye movements as compared with a procedure without eye movements. Here, Cohen's $d s$ were 0.91 and 0.66, respectively, which can be interpreted as large and medium effect sizes (see van den Hout \& Engelhard, 2012). Taken together, these findings suggest that the eye movement component of EMDR possesses a unique therapeutic potential.

\section{Proposed Working Mechanisms of EMDR}

Still, there is discussion about the working mechanism of EMDR (Landin-Romero et al., 2018). Initially, authors speculated that performing horizontal eye movements might be similar to the rapid eye movements (REM) during sleep and that both would promote a reorganization of traumatic memories and subsequent emotional processing. Others speculated that the communication between both hemispheres increases when horizontal eye movements are performed (Gunter \& Bodner, 2008). However, the beneficial effect of EMDR (i.e., reductions in subjective vividness and emotionality) has also been documented with vertical eye movements, a finding that contradicts the interhemispheric communication hypothesis that emphasizes the unique therapeutic value of horizontal eye movements (Gunter \& Bodner, 2008). More recently, de Voogd et al. (2018) found tentative evidence that eye movements may reduce amygdala activity, which could be a biological explanation for the observed reduction in emotionality.

An explanation for the mechanism underlying EMDR that has gained some empirical support is the working memory (WM) account (Gunter \& Bodner, 2008; van den Hout \& Engelhard, 2012). According to this account, retrieving a trauma memory while simultaneously performing eye movements can be seen as a dual task that taxes WM. The capacity of the WM is limited (Baddeley, 1998), and so the consequence of dual task performance is that it interferes with the full-blown retrieval of negative memories. Thus, according to the WM account, the combination of memory retrieval and simultaneously performing eye movements will lead to a degraded version - in terms of vividness and emotionality - of the trauma memory (i.e., imagination deflation; van den Hout \& Engelhard, 2012). As a result, the traumatic memory will become less emotional and this degraded version is subsequently stored during reconsolidation. 


\section{Alternative Dual Tasks}

Although eye movements are considered to be an essential ingredient of EMDR (Shapiro \& Laliothis, 2015), alternative dual tasks (e.g., bilateral tones, tapping) are also used in current clinical practice because some patients prefer performing such activities to eye movements (van den Hout et al., 2012). To the extent that alternative dual tasks are as effective as eye movements in reducing vividness and emotionality, eye movements are not contributing in a unique way to the therapeutic effects of EMDR and this may cast doubts on the central role typically ascribed to eye movements (Shapiro, 1989; Solomon \& Shapiro, 2008).

Gunter and Bodner (2008) reasoned that the WM account would predict any dual task to reduce the vividness and emotionality of memories as long as the task sufficiently taxes WM. To test this notion, van den Hout and colleagues (2010) investigated whether counting during recall taxed WM. Participants were asked to retrieve negative autobiographical memories and to rate them on vividness and emotionality. Following this, they participated in the following conditions: simple counting (subtracting 2 from 450), complex counting (subtracting 7 from 450), or a control condition (no counting). During counting, participants also performed a reaction time task (i.e., responding to presented letters) as a manipulation check to assess to what extent the alternative dual tasks taxed their WM. Afterwards, participants retrieved the negative autobiographical memories once more and again rated them on vividness and emotionality. Manipulation checks indicated that these dual tasks indeed did tax WM. Both counting conditions were associated with decreased memory vividness and emotionality ratings as compared to the control condition.

Other studies have found less convincing evidence for vividness and emotionality reductions due to alternative dual tasks. For instance, van den Hout, Muris et al. (2001) asked participants to recall positive or negative autobiographical memories and then assigned them to an eye movement, finger tapping (i.e., tapping the table top with one's index finger), or a control condition. Subsequently, the memory was retrieved again and memory vividness and emotionality were rated once more. Van den Hout et al. (2001) found that only in the eye movement condition, memory vividness and emotionality decreased.

\section{The Current Meta-Analysis}

In sum, studies have yielded inconsistent results regarding the effectiveness of alternative dual tasks in reducing vividness and emotionality of aversive memories. To address this issue in a more systematic way, we conducted a meta-analysis. Our goal was two-fold: (1) to 


\section{Chapter 3}

provide an update of laboratory studies to the meta-analysis of Lee and Cuijpers (2013); and (2) to specifically examine the effect of alternative dual tasks on vividness and emotionality of autobiographical memories. As the current meta-analysis had different in- and exclusion criteria (see below), not all laboratory studies of Lee and Cuijpers (2013) were included. Our meta-analysis might be informative for clinicians, because if we would find, for example, that eye movements do not outperform alternative dual tasks in reducing vividness and emotionality, there is at least empirical justification for exploring the use of alternative dual tasks in clinical settings (e.g., when patients prefer an alternative dual task such as bilateral tones or tapping).

\section{METHOD}

\section{Literature Search and Selection of Studies}

We used a systematic review method following the Preferred Reporting Items for Systematic Reviews and Meta-Analyses (PRISMA) statement (Moher, Liberati, Tetzlaff, Altman, \& Group, 2009; see https://osf.io/qp67t for the completed PRISMA checklist).

Our literature search was conducted in the last two weeks of January 2019. Figure 3.1 shows a PRISMA flow-chart for the selection of eligible studies. The databases PsycINFO and Web of Science were queried using the following keywords: (EMDR OR eye movement desensitization OR eye movement desensitization therapy OR eye movement desensitization and reprocessing) AND (eye movements OR dual task OR bilateral stimulation OR dual taxation). An initial number of 2852 records was found. After removing duplicates, 1780 articles were screened. We excluded: (i) review articles, commentaries, books, and dissertations; (ii) studies in which physiological manipulations were carried out or embedded in the eye movement condition (e.g., EEG, ERP's, etc.); and (iii) case reports or treatment studies (i.e., whole EMDR protocol).

We only included articles that addressed negative autobiographical memories. This type of personal recollections seems to be the best proxy of traumatic memories in patients with PTSD. More specifically, we excluded the studies of Andrade et al. (1997; black/ white newspaper pictures), Littel, Remijn, Tinga, Engelhard, and van den Hout (2017; neutral memories), Nieuwenhuis et al. (2013; words), Leer et al. (2017; faces), Engelhard, van den Hout, and Smeets (2011; participants who watched the Queen's Day tragedy via television), and Houben, Otgaar, Roelofs, and Merckelbach (2018; participants watched a trauma film). 


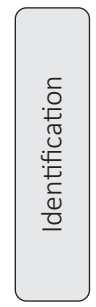

Total of records identified through database searching and additional records identified through other sources

$(n=2852)$

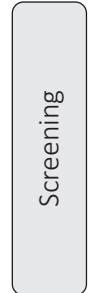

Records after duplicates removed $(n=1780)$

Full-text articles excluded, with reasons* ( $n=15)$

- No negative idiosyncratic materials (i.e., autobiographical memories) $(\boldsymbol{k}=\mathbf{8})$

- No visual analogue scale for vividness and emotionality $(\boldsymbol{k}=\mathbf{3})$ - Patient studies $(\boldsymbol{k}=\mathbf{4})$
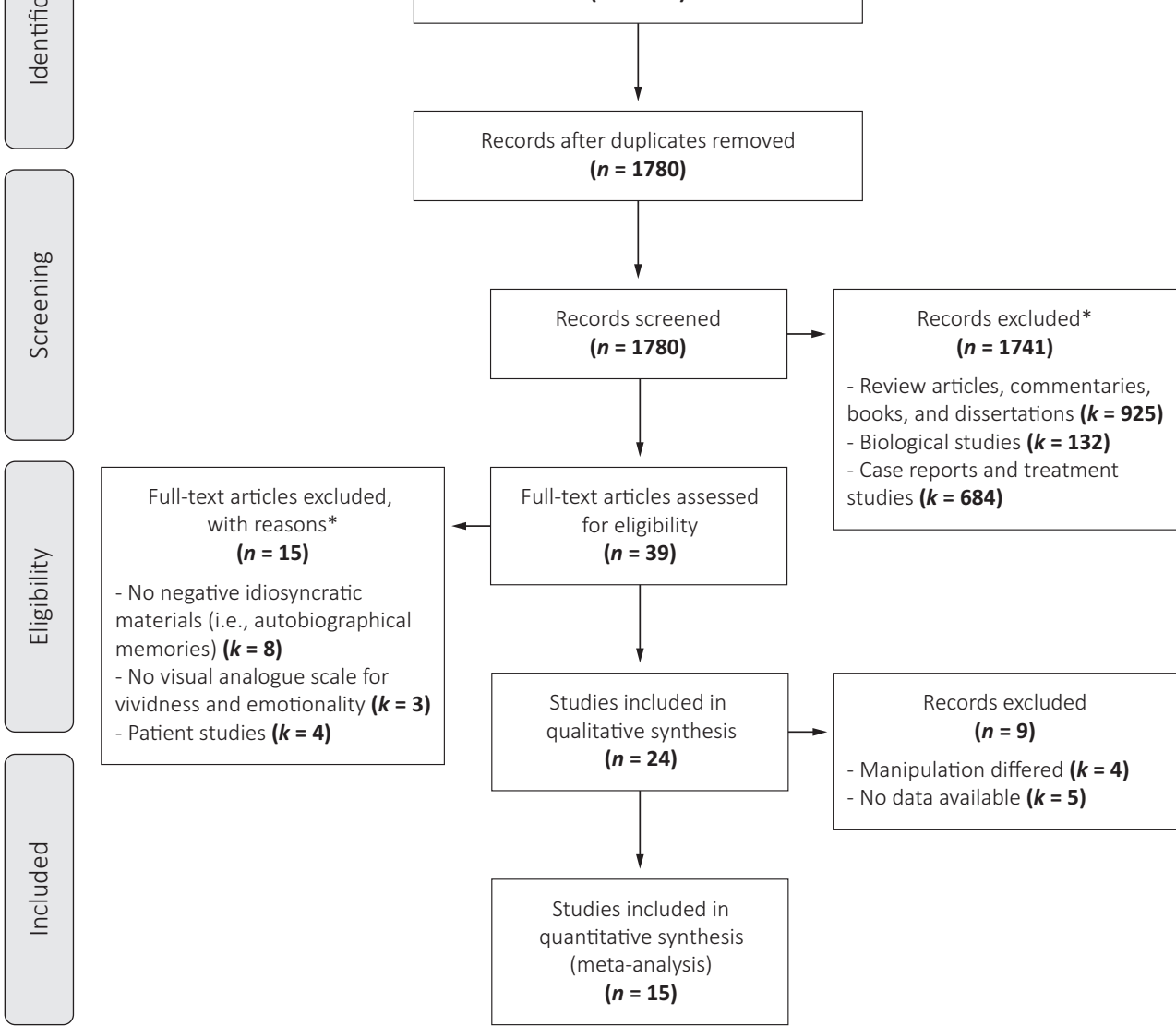

$(n=39)$

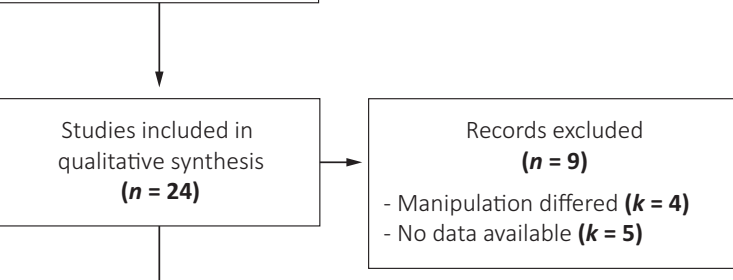

Studies included in quantitative synthesis

(meta-analysis)

$(n=15)$

Figure 3.1 PRISMA flow chart diagram of meta-analysis study selection. * = articles may have met more than one exclusion criteria.

We further excluded two studies that focused on flash-forwards (i.e., future-oriented images; Engelhard, van den Hout, Janssen, \& van der Beek, 2010; Engelhard, van den Hout, Dek et al., 2011). In addition, we excluded studies that did not rely on a visual analogue scale (VAS) to measure vividness and emotionality (Christman, Garvey, Propper, \& Phaneuf, 2003; Greenwald, McClintock, \& Bailey, 2013; Greenwald, McClintock, Jarecki, \& Monaco, 2015) 


\section{Chapter 3}

and/or that solely relied on patient data (Thomaes et al., 2016; de Jongh, Ernst, Marques, \& Hornsveld, 2013; Lilley, Andrade, Turpin, Sabin-Farrell, \& Holmes, 2009; Matthijssen, Verhoeven, van den Hout, \& Heitland, 2017). A number of additional studies were identified by manual searches of review articles (e.g., Lee \& Cuijpers, 2013, van den Hout \& Engelhard, 2012). This initial search resulted in 24 potentially eligible articles. However, four of these studies were discarded because the manipulation (i.e., eye movements, alternative dual task, or control instruction) also involved some other task (e.g., presentations of screenshots of event, letter identification during eye movements, a manipulation of the knowledge about EMDR; Cuperus, Laken, van Schie, Engelhard, \& van den Hout, 2019; Kavanagh, Freese, Andrade, \& May, 2001; Onderdonk \& van den Hout, 2016; Littel, van Schie, \& van den Hout, 2017). And five further studies had to be excluded because of missing data, although we did make an attempt to obtain the data by sending the first and/or second authors of these papers a request to share their data (van den Hout, Engelhard, Beetsma et al., 2011; van den Hout et al., 2012; van den Hout, Bartelski, \& Engelhard, 2013; Gunter \& Bodner, 2008; Hornsveld et al., 2010). Datasets from four studies were unavailable, the authors of the remaining study did not reply.

This resulted in a total of 15 studies that described 16 separate experiments and included 942 research participants. Thirteen of these studies (including 14 experiments and 867 participants) involved a comparison between eye movement and control conditions (see Table 3.1). Seven studies (including eight experiments and 353 participants) involved a comparison between alternative dual tasks and control conditions (see Table 3.2). These studies used a range of alternative dual tasks, including the shape sorter task, playing a Tetris game, binaural stimulation (beeps), simple counting (subtracting 2 from 450), complex counting (subtracting 7 from 450), tapping, articulatory suppression (counting from 1), and listening to counting (1-60). In all studies, the duration of the alternative dual task was similar to the duration of eye movements (e.g., $4 \times 24$ s; see Table 3.2). For direct comparisons involving alternative dual tasks and eye movements (see Table 3.3), we only included studies that comprised both conditions. Here, five studies describing six experiments and 278 participants were eligible. 
The effects of eye movements and alternative dual tasks on the vividness and emotionality of autobiographical memory

Table 3.1 Overview Studies Included Comparison Eye Movements versus Control.

\begin{tabular}{|c|c|c|c|}
\hline Author (year) & Sample (n) & Duration of EM & Design \\
\hline Barrowcliff, Gray, Freeman, \& Macculloch, 2004 & $\begin{array}{l}80 \text { community participants } \\
\text { and undergraduates }\end{array}$ & $1 \mathrm{~Hz}$ for $25 \mathrm{~s}$ & WS \\
\hline Engelhard et al., 2012 & 29 undergraduates & $6 \times 24 s$ & WS \\
\hline Engelhard, van Uijen, \& van den Hout, 2010 & 60 undergraduates & $4 \times 24 s$ & WS \\
\hline $\begin{array}{l}\text { van den Hout, Engelhard, Rijkeboer et al., } 2011 \\
\text { (Exp. 4) }\end{array}$ & 54 undergraduates & $4 \times 24 s$ & WS \\
\hline van den Hout et al., 2001 & 30 undergraduates & $4 \times 24 s$ & WS \\
\hline Kemps \& Tiggemann, 2007 (Exp. 1) & 30 undergraduates & One eye movement per s & WS \\
\hline Kemps \& Tiggemann, 2007 (Exp. 2) & 68 undergraduates & One eye movement per s & WS \\
\hline Kristjánsdottir \& Lee, 2011 & $\begin{array}{l}36 \text { community participants } \\
\text { and undergraduates }\end{array}$ & $\begin{array}{l}\text { One eye movement per } \\
\text { s for } 60 \mathrm{~s}\end{array}$ & WS \\
\hline Leer, Engelhard, \& van den Hout, 2014 & 73 undergraduates & $\begin{array}{c}4 \times 24 \text { s and } \\
8 \times 24 s\end{array}$ & WS \\
\hline Mertens et al., 2019 & 100 undergraduates & $4 \times 24 s$ & WS \\
\hline van Schie et al., 2016 & 66 undergraduates & $6 \times 24 \mathrm{~s}(0.8 \mathrm{~Hz}$ or $1.2 \mathrm{~Hz})$ & WS \\
\hline $\begin{array}{l}\text { Smeets, Dijs, Pervan, Engelhard, \& van den } \\
\text { Hout, } 2012\end{array}$ & 61 undergraduates & $\begin{array}{c}4 \times 24 \text { s, repeated three } \\
\text { times }\end{array}$ & BS \\
\hline van Veen, Engelhard, \& van den Hout, 2016 & 108 undergraduates & $8 \times 24 s$ & BS \\
\hline van Veen et al., 2015 (Exp. 2) & 72 undergraduates & $6 \times 24 \mathrm{~s}(0.8 \mathrm{~Hz}$ or $1.2 \mathrm{~Hz})$ & WS \\
\hline
\end{tabular}

Notes. EM = Eye Movements; WS = Within Subjects design; BS = Between Subjects design.

Table 3.2 Overview Studies Included Comparison Alternative Dual Task versus Control.

\begin{tabular}{|c|c|c|c|}
\hline Author (year) & Sample (n) & Alternative dual task and Duration & Design \\
\hline $\begin{array}{l}\text { Cuperus, Laken, van den Hout, \& Engelhard, } \\
2016 \text { (Exp. 2) }\end{array}$ & 34 undergraduates & Shape sorter task $(4 \times 24$ s) & BS \\
\hline Engelhard, van Uijen, \& van den Hout, 2010 & 60 undergraduates & Tetris $(4 \times 24 \mathrm{~s})$ & WS \\
\hline $\begin{array}{l}\text { van den Hout, Engelhard, Rijkeboer et al., } 2011 \\
\text { (Exp. 4) }\end{array}$ & 54 undergraduates & Binaural stimulation $(4 \times 24$ s) & WS \\
\hline van den Hout et al., 2010 & 41 undergraduates & $\begin{array}{l}\text { Simple and complex counting } \\
\qquad(4 \times 24 \mathrm{~s})\end{array}$ & WS \\
\hline van den Hout et al., 2001 & 30 undergraduates & Tapping (4 × 24 s) & WS \\
\hline Kemps \& Tiggemann, 2007 (Exp. 1) & 30 undergraduates & Articulatory suppression (n.s.) & WS \\
\hline Kemps \& Tiggemann, 2007 (Exp. 2) & 68 undergraduates & Articulatory suppression (n.s.) & WS \\
\hline Kristjánsdottir \& Lee, 2011 & $\begin{array}{l}36 \text { community } \\
\text { participants and } \\
\text { undergraduates }\end{array}$ & Counting (60 s) & WS \\
\hline
\end{tabular}

Notes. BS = Between Subjects; WS = Within Subjects. n.s. = not specified 


\section{Chapter 3}

Table 3.3 Overview Studies Included Comparison Alternative Dual Tasks versus Eye Movements.

\begin{tabular}{|c|c|c|c|c|}
\hline Author (year) & Sample (n) & EM duration & Alternative dual task and Duration & Design \\
\hline $\begin{array}{l}\text { Engelhard, van Uijen, \& van } \\
\text { den Hout, } 2010\end{array}$ & 60 undergraduates & $4 \times 24 s$ & Tetris $(4 \times 24 \mathrm{~s})$ & WS \\
\hline $\begin{array}{l}\text { van den Hout, Engelhard, } \\
\text { Rijkeboer et al., } 2011 \text { (Exp. 4) }\end{array}$ & 54 undergraduates & $4 \times 24 s$ & Binaural stimulation $(4 \times 24$ s) & WS \\
\hline van den Hout et al., 2001 & 30 undergraduates & $4 \times 24 s$ & Tapping $(4 \times 24 \mathrm{~s})$ & WS \\
\hline $\begin{array}{l}\text { Kemps \& Tiggemann, } 2007 \\
\text { (Exp. 1) }\end{array}$ & 30 undergraduates & $\begin{array}{l}\text { One eye } \\
\text { movement per s }\end{array}$ & Articulatory suppression (n.s.) & WS \\
\hline $\begin{array}{l}\text { Kemps \& Tiggemann, } 2007 \\
\text { (Exp. 2) }\end{array}$ & 68 undergraduates & $\begin{array}{l}\text { One eye } \\
\text { movement per s }\end{array}$ & Articulatory suppression (n.s.) & WS \\
\hline Kristjánsdottir \& Lee, 2011 & $\begin{array}{l}36 \text { community } \\
\text { participants and } \\
\text { undergraduates }\end{array}$ & $\begin{array}{l}\text { One eye } \\
\text { movement per s } \\
\text { for } 60 \mathrm{~s}\end{array}$ & Counting (60 s) & WS \\
\hline
\end{tabular}

Notes. EM = Eye Movement BS = Between Subjects; WS = Within Subjects; n.s. = not specified.

\section{Meta-analytic Procedure}

Cohen's $d$ was chosen as the effect size indicator, because we focused on two experimental conditions (i.e., eye movements and alternative dual tasks) that each were contrasted with a control condition (memory recall-only). For each study, Cohen's $d$ was calculated by subtracting the average post-test score of the control condition from the average post-test score of the experimental condition and dividing the result by the pooled standard deviations of post-intervention scores as reported for the control and experimental conditions. Effect sizes were calculated for the following contrasts: (1) eye movements versus control; (2) alternative dual tasks versus control; and (3) eye movements versus alternative dual tasks. Mean Cohen's ds were calculated for vividness and emotionality separately. Effect sizes of 0.80 and higher were interpreted as large, effect sizes of 0.5 - 0.8 as medium, and effect sizes below 0.5 as small (Cohen, 1988). Several studies included multiple experimental conditions, for example performing eye movements at different speeds (van Schie et al., 2016; van Veen et al., 2015), individual differences in WM capacity (e.g., van Schie et al., 2016), or type of memory (i.e., visual versus auditory; Kemps \& Tiggemann, 2007). Hence, multiple effect sizes were obtained for these studies (e.g., slow EM vs. control and fast EM vs. control). Forrest plots were constructed using the statistical software package JASP (JASP Team, 2019; version 0.11.1). For data file and analysis, see https://osf.io/5f6d2.

Additionally, heterogeneity analyses were performed by calculating $R^{2}$ with JASP (JASP Team, 2019). The $I^{2}$ index can be interpreted as the percentage (range 0-100\%; Higgins \& Thompson, 2002) of the total variability between studies. Specifically, it is an indicator of 
what proportion of the observed variance would remain if sampling error variability across studies would be eliminated (Borenstein, Higgins, Hedges, \& Rothstein, 2017). A low index (< $25 \%)$ means that variability in effect size estimates are mainly due to sampling error, whereas high indices (>75\%) imply true heterogeneity across studies (Higgins \& Thompson, 2002).

Publication bias was tested by inspecting the funnel plot on the primary outcome measures (JASP Team, 2019). A funnel plot is a visual representation of study precision (1/SE) against the observed effect size (ES). In the absence of publication bias, the plot is expected to be symmetrical in the vertical axis around the meta-analytic compounded effect size estimate. The points that represent the studies should lie in a confidence triangle of 95\%. Points outside this interval can result from heterogeneity and/or study differences. Asymmetry of the funnel plot is regarded as evidence for publication bias (Grasman, 2017).

\section{RESULTS}

Effects on Vividness. The mean effect size (Cohen's d; see Table 3.4) for the difference between eye movements and the control condition was 0.59 (95\% $\mathrm{Cl}[0.42,0.77]$, see Figure 3.2a). For the comparison between alternative dual tasks and control, a Cohen's $d$ of 0.49 (95\% Cl [0.14, 0.85], see Table $\mathbf{3 . 4}$ and Figure 3.2b) was obtained. The mean effect size for the difference between eye movements and alternative dual tasks was 0.29 and the lower part of its associated confidence interval came close to zero (95\% Cl [0.03, 0.56], see Table 3.4 and Figure 3.2c). All comparisons were associated with substantial heterogeneity. More specifically, we found heterogeneity values ( $12 \%)$ of $75.24 \%(95 \% \mathrm{Cl}[57.99,89.52]), 84.74 \%(95 \% \mathrm{Cl}[67.28,95.96])$, and $66.61 \%(95 \% \mathrm{Cl}[20.55,92.35])$ for eye movements versus control, alternative dual tasks versus control, and eye movements versus alternative dual tasks, respectively.

Table 3.4 Mean Cohen's $d$, 95\% Confidence Interval and Heterogeneity.

\begin{tabular}{|c|c|c|c|c|c|c|c|c|}
\hline \multirow[t]{2}{*}{ Comparison } & \multicolumn{4}{|c|}{ Vividness } & \multicolumn{4}{|c|}{ Emotionality } \\
\hline & $\begin{array}{c}\text { Mean } \\
\text { Cohen's } d\end{array}$ & $95 \% \mathrm{Cl}$ & $P^{2}(\%)$ & $95 \% \mathrm{Cl}$ of $P^{2}$ & $\begin{array}{c}\text { Mean } \\
\text { Cohen's } d\end{array}$ & $95 \% \mathrm{Cl}$ & $P^{2}(\%)$ & $95 \% \mathrm{Cl}$ of $l^{2}$ \\
\hline EM vs. Control & 0.59 & {$[0.42,0.77]$} & 75.24 & {$[57.99,89.52]$} & 0.28 & {$[0.15,0.42]$} & 56.01 & {$[27.24,83.42]$} \\
\hline $\begin{array}{l}\text { Alternative Dual } \\
\text { Tasks vs. Control }\end{array}$ & 0.49 & {$[0.14,0.85]$} & 84.74 & {$[67.28,95.96]$} & 0.32 & {$[0.14,0.50]$} & 42.62 & {$[0.00,78.72]$} \\
\hline $\begin{array}{l}\text { Alternative Dual } \\
\text { Tasks vs. EM }\end{array}$ & 0.29 & {$[0.03,0.56]$} & 66.61 & {$[20.55,92.35]$} & 0.02 & {$[-0.27,0.31]$} & 72.81 & {$[33.61,94.65]$} \\
\hline
\end{tabular}

Notes. $\mathrm{EM}=$ Eye Movements; $\mathrm{Cl}=$ Confidence interval; ${ }^{2}$ = heterogeneity. 


\section{Chapter 3}

(a)

Barrowcliff et al., 2004

Engelhard et al., 2012

Engelhard et al., 2010

van den Hout et al., 2011 (Exp. 4)

van den Hout et al., 2001

Kemps \& Tiggemann, 2007 (Exp. 1)

Kemps \& Tiggemann, 2007 (Exp. 2). 1

Kemps \& Tiggemann, 2007 (Exp. 2). 2

Kristjansdottir \& Lee, 2011

Leer et al., 2014.1

Leer et al., 2014.2

Mertens et al., 2019

van Schie et al., 2016.1

van Schie et al., 2016.2

van Schie et al., 2016.3

van Schie et al., 2016.4

Smeets et al., 2012

van Veen et al., 2016.1

van Veen et al., 2016.2

van Veen et al., 2015 (Exp. 2).1

van Veen et al., 2015 (Exp. 2).2

\begin{tabular}{|c|c|c|c|c|c|c|}
\hline \multirow[t]{3}{*}{ RE Model } & & & & & & $0.59[0.42,0.77]$ \\
\hline & $\Gamma$ & iं & $T$ & $T$ & 7 & \\
\hline & -1 & 0 & 1 & 2 & 3 & \\
\hline
\end{tabular}

(b)

Cuperus et al., 2016

Engelhard et al., 2010

van den Hout et al., 2011 (Exp. 4)

van den Hout et al., 2010.1

van den Hout et al., 2010.2

van den Hout et al., 2001

Kemps \& Tiggemann (Exp. 1)

Kemps \& Tiggemann (Exp. 2).1

Kemps \& Tiggemann (Exp. 2).2

Kristjansdottir \& Lee, 2011
$0.36[0.05,0.67]$

$0.63[0.11,1.16]$

$0.16[-0.20,0.52]$

$0.75[0.36,1.14]$

$0.52[0.01,1.04]$

$1.06[0.52,1.60]$

$1.45[1.07,1.83]$

$0.77[0.42,1.12]$

$2.03[1.47,2.60]$

$0.45[0.13,0.78]$

$0.47[0.15,0.80]$

$0.26[-0.02,0.54]$

$0.54[0.03,1.05]$

$0.42[-0.09,0.92]$

$0.61[0.13,1.09]$

$0.57[0.09,1.04]$

$0.61[0.10,1.12]$

$0.33[-0.14,0.79]$

$-0.01[-0.47,0.45]$

$0.35[0.02,0.68]$

$0.52[0.19,0.86]$

$0.59[0.42,0.77]$

RE Model

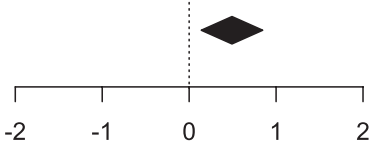

$0.49[0.14,0.85]$

Observed Outcome 
(c)

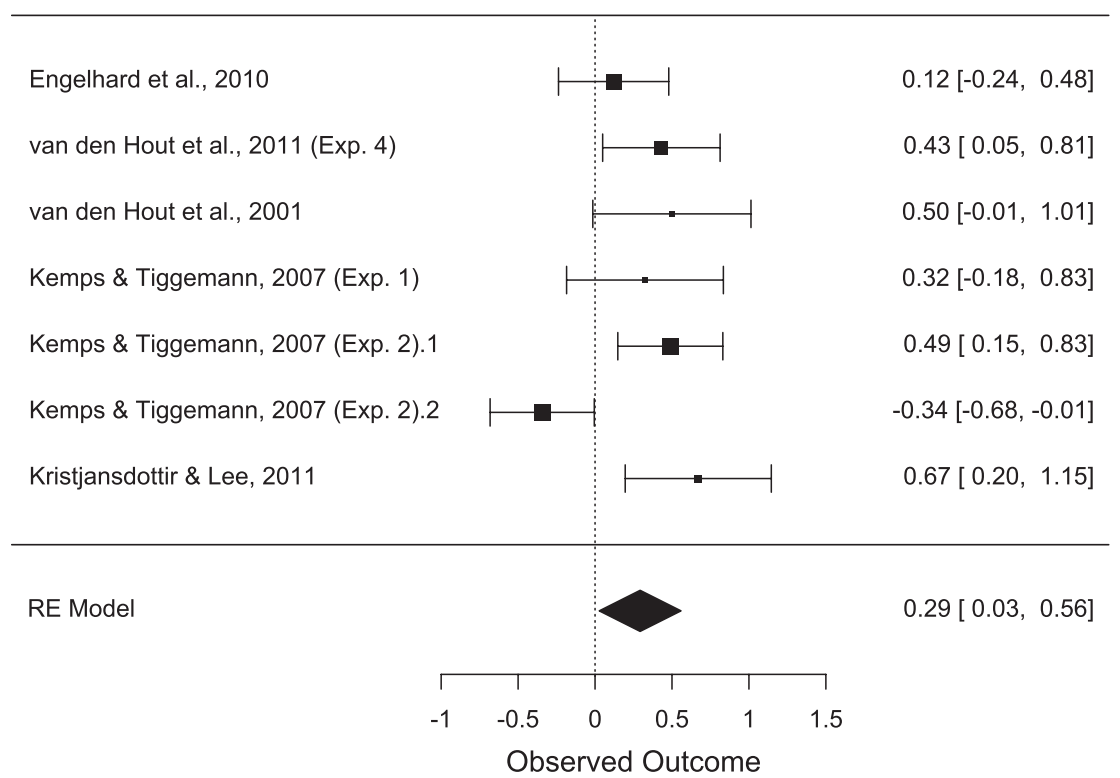

Figure 3.2 Forest Plot for (a) Eye Movements versus Control, (b) Alternative Dual Tasks versus Control and (c) Eye Movements versus Alternative Dual Tasks for Vividness. RE Model = Random-Effects model.

Effects on Emotionality. A Cohen's $d$ of 0.28 (95\% Cl [0.15, 0.42]) was found for the comparison between eye movements and control (see Table $\mathbf{3 . 4}$ and Figure 3.3a). For the difference between alternative dual tasks and control, a mean Cohen's $d$ of 0.32 (95\% Cl $[0.14,0.50]$, see Table 3.4 and Figure 3.3b) was found. A Cohen's $d$ of 0.02 (95\% Cl $[-0.27,0.31])$ was found for the comparison between eye movements and alternative dual tasks (see Table $\mathbf{3 . 4}$ and Figure 3.3c). Moderate heterogeneity values ( $12 \%$; $56.01 \%(95 \% \mathrm{Cl}[27.24,83.42])$ and $42.62 \%(95 \% \mathrm{Cl}[0.00,78.72])$ were found for the first two comparisons, whereas a substantial heterogeneity $(72.81 \%, 95 \% \mathrm{Cl}[33.62,94.65])$ was evident for the comparison between eye movements and alternative dual tasks.

Publication Bias. Funnel plots can be checked at https://osf.io/bwe29. Neither visual inspection of the funnel plots nor the trim and fill procedure (Duval \& Tweedie, 2000) identified substantial asymmetry. Most studies were projected within the confidence triangle. The few studies that fell outside the triangle tended to rely on a low sample size. 


\section{Chapter 3}

(a)

Barrowcliff et al., 2004

Engelhard et al., 2012

Engelhard et al., 2010

van den Hout et al., 2011 (Exp. 4)

van den Hout et al., 2001

Kemps \& Tiggemann, 2007 (Exp. 1)

Kemps \& Tiggemann, 2007 (Exp. 2). 1

Kemps \& Tiggemann, 2007 (Exp. 2).2

Kristjansdottir \& Lee, 2011

Leer et al., 2014.1

Leer et al., 2014.2

Mertens et al., 2019

van Schie et al., 2016.1

van Schie et al., 2016.2

van Schie et al., 2016.3

van Schie et al., 2016.4

Smeets et al., 2012

van Veen et al., 2016.1

van Veen et al., 2016.2

van Veen et al., 2015 (Exp. 2).1

van Veen et al., 2015 (Exp. 2).2

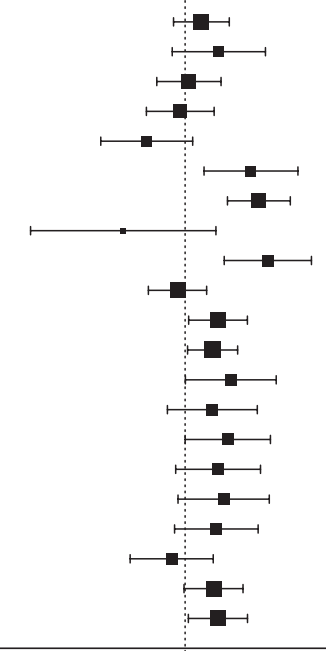

$0.18[-0.13,0.49]$

$0.38[-0.14,0.89]$

$0.04[-0.32,0.40]$

$-0.05[-0.43,0.32]$

$-0.43[-0.94,0.08]$

$0.73[0.21,1.26]$

$0.82[0.47,1.17]$

$-0.69[-1.72,0.34]$

$0.92[0.44,1.41]$

$-0.08[-0.41,0.24]$

$0.37[0.04,0.69]$

$0.31[0.03,0.58]$

$0.51[0.00,1.01]$

$0.30[-0.20,0.80]$

$0.48[0.00,0.95]$

$0.37[-0.10,0.84]$

$0.43[-0.08,0.94]$

$0.35[-0.12,0.81]$

$-0.15[-0.61,0.31]$

$0.32[-0.01,0.64]$

$0.37[0.04,0.69]$

RE Model

$0.28[0.15,0.42]$

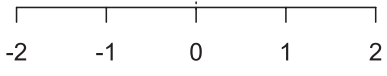

Observed Outcome

(b)

Cuperus et al., 2016

Engelhard et al., 2010

van den Hout et al., 2011 (Exp. 4)

van den Hout et al., 2010.1

van den Hout et al., 2010.2

van den Hout et al., 2001

Kemps \& Tiggemann (Exp. 1)

Kemps \& Tiggemann (Exp. 2).1

Kemps \& Tiggemann (Exp. 2).2

Kristjansdottir \& Lee, 2011

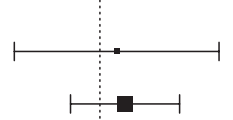

$0.11[-0.56,0.78]$

$0.17[-0.19,0.52]$

$0.18[-0.20,0.55]$

$0.14[-0.29,0.58]$

$0.22[-0.21,0.66]$

$0.07[-0.43,0.58]$

$0.42[-0.09,0.94]$

$0.36[0.02,0.70]$

$0.93[0.58,1.28]$

$0.40[-0.06,0.87]$

$0.32[0.14,0.50]$

RE Model

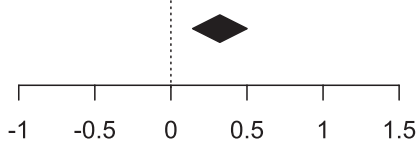

Observed Outcome 
(c)

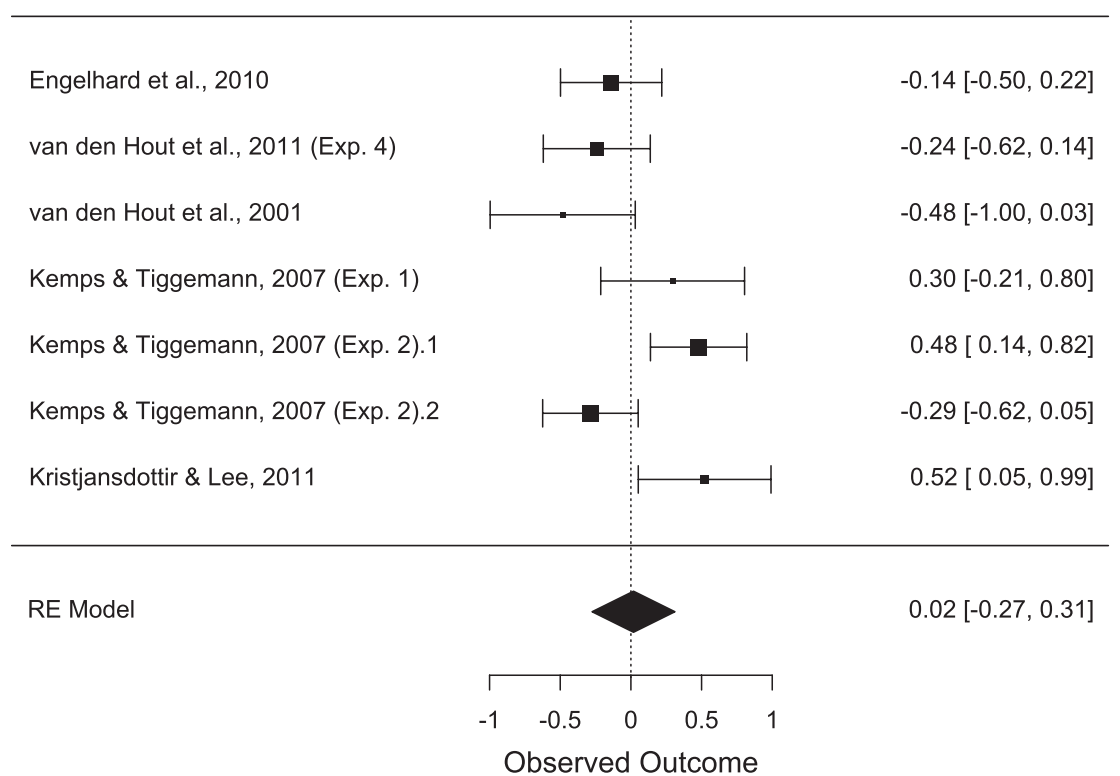

Figure 3.3 Forest Plot for (a) Eye Movements versus Control, (b) Alternative Dual Tasks versus Control and (c) Eye Movements versus Alternative Dual Tasks for Emotionality. RE Model = Random-Effects model

\section{DISCUSSION}

Using a meta-analytic approach, we compared to what extent eye movements and alternative dual tasks reduce the vividness and emotionality of aversive memories. The main results can be summarized as follows. First, we found that eye movements and alternative dual tasks produced similar reductions in vividness and emotionality in comparison with control conditions. The current meta-analysis obtained a medium effect size (Cohen's $d=0.59$ ) for vividness and a small effect size (Cohen's $d=0.28$ ) for emotionality when eye movements were contrasted against control conditions. This is in line with the findings of Lee and Cuijpers (2013), who found a larger effect size for vividness (Cohen's $d=0.91$ ) than for emotionality (Cohen's $d=0.66$ ) when they compared eye movements and control conditions of laboratory studies. Because both meta-analyses had different in- and exclusion criteria (e.g., randomized controlled trials, alternative dual task), the precise effect size values can be expected to differ. What is remarkable is that both meta-analyses obtained an overall stronger effect of eye movements on vividness than on emotionality. In the current study, a similar pattern emerged when alternative dual tasks were compared with control conditions. We found a 


\section{Chapter 3}

medium effect size (Cohen's $d=0.49$ ) for vividness and a small effect size (Cohen's $d=0.32$ ) for emotionality. Second, when eye movements were compared to alternative dual tasks, small effect sizes for both vividness (Cohen's $d=0.29$ ) and emotionality (Cohen's $d=0.02$ ) were found. Although this indicates that eye movements reduce vividness more effectively than alternative dual tasks, we do not know whether the modest effect size associated with this difference is clinically relevant. Establishing such a clinically noticeable effect would require another type of metric (e.g., "minimal important difference"; Cuijpers, Turner, Koole, van Dijke, \& Smit, 2014). In addition, one of the main aims of EMDR is to reduce the emotional valence of the traumatic memory (Shapiro, 2002). Our results suggests that both approaches may be used to obtain this therapeutic effect.

Our meta-analytic results accord well with a main prediction that can be derived from the WM account (Gunter \& Bodner, 2008), namely that alternative dual tasks (that presumably tax working memory) and eye movements are both effective in reducing the vividness and emotional load of negative memories. This raises the more fundamental question whether eye movements are an essential ingredient of EMDR. Because our analysis did not involve clinical groups, our data cannot answer this question. At the very least, they provide some empirical justification for EMDR therapists using alternative dual tasks when patients indicate that it is not feasible for them to perform eye movements.

The included studies have some constraints. For example, their sample size varied considerably: The lowest samples size was $N=29$ (Engelhard et al., 2012), while the highest was $N=108$ (van Veen et al., 2016). Of course, effect sizes generated by studies with small samples have to be interpreted with caution. Also, most of the included studies (i.e., 12 out of 15) employed a within-subjects design (e.g., van Veen et al., 2015), which means that participants completed all experimental conditions. This type of design might lead to carryover effects and probably introduces some noise in the effect size estimates (Quintana, 2015). In addition, although we specifically included studies that relied on visual analogue scales of vividness and emotionality, such self-report measurements can be prone to demand characteristics (Kearns \& Engelhard, 2015; Kanter et al., 2002). Little, van Schie, and van den Hout (2017; Experiment 1) found some tentative evidence that expectancy effects drive the effectiveness of EMDR. However, in their second experiment, these authors noted that providing information about EMDR did not influence the vividness and emotionality ratings of participants. Lastly, our heterogeneity analyses showed moderate to substantial heterogeneity between studies. However, $l^{2}$ estimates should be taken as provisional estimates because 
the current meta-analysis relied on a limited number of studies (von Hippel, 2015). Though no publication bias was observed, our test might be underpowered because of the limited number of studies (Carter, Schönbrodt, Gervais, \& Hilgard, 2019).

The current meta-analysis has several shortcomings that limit its generalization to clinical settings. First, only a relatively small number of laboratory studies was included ( $k=$ 15). Note, however, that other meta-analyses in the EMDR domain included similar numbers of studies (see e.g., Khan et al., 2018; Moreno-Alcázar et al., 2017).

Second, all alternative dual tasks (e.g., tapping and counting) were combined for the analyses, which may mask an effect of modality. Therefore, we cannot conclude whether a specific alternative dual task might yield superior therapeutic effects. For example, Andrade et al. (1997) hypothesized that visuospatial tasks would be particularly effective as they tax the visuospatial sketchpad, which is the part of WM primarily used when retrieving memories. However, no modality-specific effect was found (see e.g., van Schie et al., 2019). The current meta-analysis did not evaluate the degree to which these alternative tasks tax WM. Advocates of the WM account (van den Hout \& Engelhard, 2012) proposed an inverted U-shape, which means that too little or too much taxing will produce no or little effect on the vividness and emotionality of memories. However, Littel and van Schie (2019) found no evidence for the inverted $U$-curve. Hence, more research is required to examine modality specific taxing and the amount of WM taxation.

Third, the search terms that we used for the meta-analysis are perhaps too specific and we may have missed potentially relevant studies. For example, studies have also focused on the effect of eye movements on positive memories (e.g., Engelhard, van Uijen, \& van den Hout, 2010). Although not central to the aims of the current study, we conducted exploratory analyses on these studies and a similar pattern emerged (see https://osf.io/bf2k7). That is, only small effect sizes were obtained in favour of eye movements when compared to alternative dual tasks. Unpublished datasets were requested, but either no replies were received or authors indicated that there were no unpublished datasets.

To sum up, the eye movement component of EMDR intends to diminish the emotional impact of negative memories (Shapiro, 2002). We showed that a similar effect could be obtained with alternative dual tasks in laboratory studies. Furthermore, in line with Lee and Cuijpers (2013), our current meta-analysis indicates that eye movements, or alternative dual tasks for that matter, impact vividness more strongly than the emotional load of a negative memory. This is not necessarily bad news for EMDR, because the pattern 


\section{Chapter 3}

is entirely consistent with the WM account of its therapeutic potential: eye movements or alternative dual tasks interfere with WM, which reduce the vividness of negative memories, which in turn lowers their associated negative value (Gunter \& Bodner, 2008; Smeets, Dijs, Pervan, Engelhard, \& van den Hout, 2012). The important point here is that vividness may play a more prominent role in the therapeutic effect of EMDR than initially assumed.

Although the importance of eye movements is emphasized in Dutch EMDR protocols (Beer et al., 2011), our findings do encourage EMDR therapists to explore a wider variety of alternative interventions that are perhaps equally effective in reducing the vividness and emotionality of aversive memories. EMDR theorists often assume that decreases in subjective units of distress should result in a reduction of symptomatology. However, so far no convincing link between changes in vividness and emotionality (due to eye movements or alternative dual tasks) and changes in symptomatology has been found (van Schie et al., 2019). More research on the underlying mechanisms of EMDR and on the effectiveness in reducing symptomatology is needed to draw firm conclusions about the mode of action of EMDR.

Section I focused on instructions in the Dutch EMDR protocol. Section II will focus on the legal psychological effects of EMDR. As (traumatic) memory is a reconstructive process, which can lead to memory errors, it is imperative that EMDR practitioners are knowledgeable about the functioning of memory. In the next chapter, EMDR practitioners, psychology students and memory researchers were questioned on several correct and controversial statements about memory. In the following chapters, experimental studies will be presented on the link between eye movements and false memories. 


SECTION II 


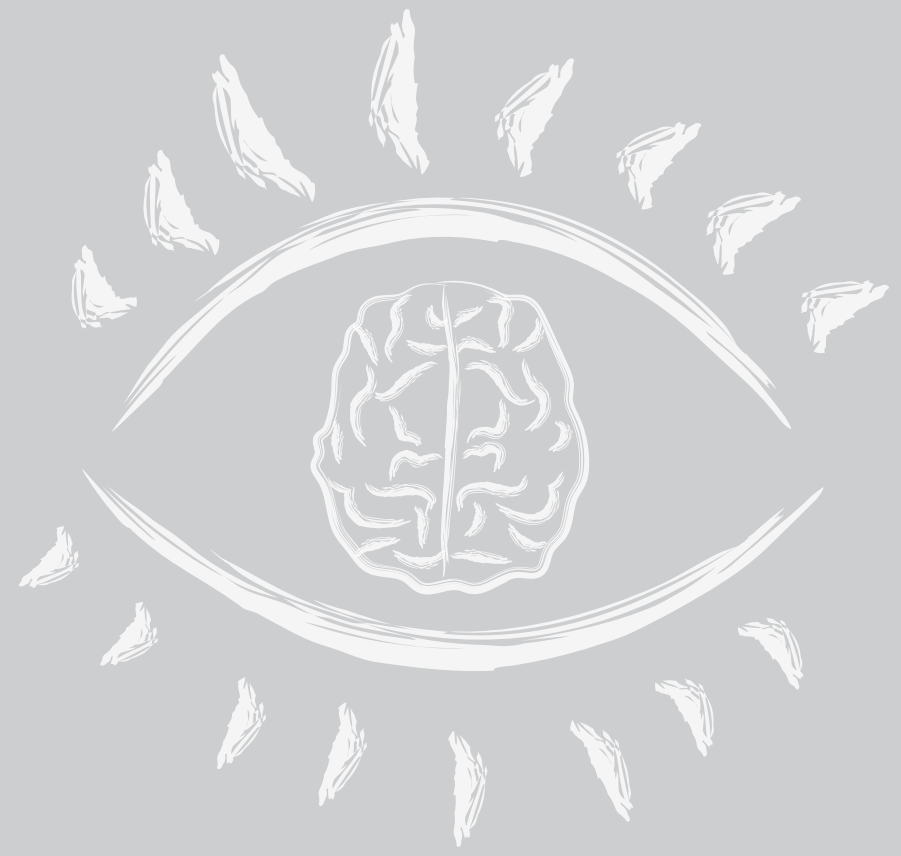




\section{CHAPTER 4}

\section{Eye Movement Desensitization and Reprocessing practitioners' beliefs about memory}

Published as:

Houben, S. T. L., Otgaar, H., Roelofs, J., Wessel, I., Patihis, L., \& Merckelbach, H. (2019). Eye movement desensitization and reprocessing (EMDR) practitioners' beliefs about memory. Psychology of Consciousness: Theory, Research, and Practice. https://doi.org/10.1037/cns0000211 



\section{INTRODUCTION}

In classical Eye Movement Desensitization and Reprocessing (EMDR; Shapiro, 1989), the therapist's index finger moves horizontally in front of the patient's visual field while the patient's eyes follow the therapist's finger. Simultaneously, the patient tries to recollect the most intrusive part of a traumatic memory. Meta-analytic research has suggested that the joint task of recalling the traumatic memory and following the therapist's finger mitigates the vividness and emotionality linked to the traumatic memory (Lee \& Cuijpers, 2013; but see Devilly, Ono, \& Lohr, 2014). Given these positive results, the World Health Organization (Born, Rasch, \& Gais, 2013) has selected EMDR as a treatment of choice for post-traumatic stress disorder (PTSD). Originally developed as an intervention for people with PTSD, EMDR is nowadays applied to a wide range of (mental) health problems in which aversive memories or experiences play a role (de Jongh et al., 2013; Rikkert, van Rood, de Roos, Ratter, \& van den Hout, 2018). Research interest in EMDR has also increased. We searched the database PsycINFO using the entry terms "emdr OR eye movement desensitization OR eye movement desensitization therapy OR eye movement desensitization and reprocessing" from the year of Shapiro's first publication (1989) until 2018. As can be seen in Figure 4.1, EMDR publications have increased over the years, which testify to the scientific interest in the intervention as well the mechanisms that may underlie its effect.

Studies examining the efficacy of treatments such as EMDR often focus on positive outcomes, thereby overlooking the potential of negative effects. To facilitate research on such

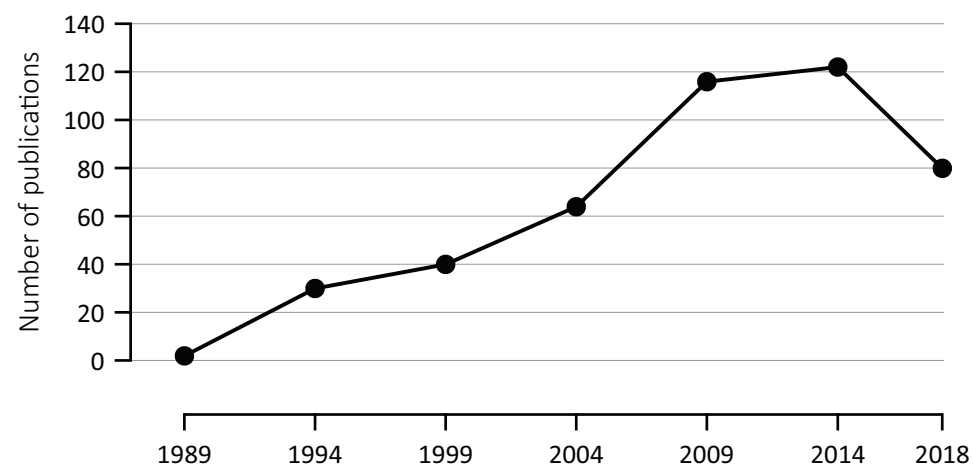

Figure 4.1 Number of (international) publications on EMDR found in PsycINFO. Search terms included "emdr OR eye movement desensitization OR eye movement desensitization therapy OR eye movement desensitization and reprocessing" per year. 


\section{Chapter 4}

potential negative effects, Rozental, Kottorp, Boettcher, Andersson, and Carlbring (2016) developed an instrument with which they surveyed participants ( $N=653)$ who received smartphone delivered self-help treatment or individuals who in the past had undergone psychological treatment. One negative side effect of treatment that was relatively often mentioned was the resurfacing of unpleasant memories. This finding is also relevant for EMDR practitioners, because there are reasons to assume that EMDR has the potential to elicit false memories (i.e., memories of non-existing events; Houben et al., 2018; Lohr et al., 1998; Muris \& Merckelbach, 1999). For example, Houben and colleagues (2018) used the misinformation paradigm (Loftus et al., 1978) to examine the susceptibility to suggestionbased false memories after performing eye movements. Participants viewed a video of a car crash and following this, performed eye movements or not while thinking about the video. Afterwards, they received misinformation and were tested on their memory. Participants in the eye movement condition reported more misinformation than participants in the control condition. Thus, eye movements, as used in EMDR, can undermine memory integrity. However, this finding should be interpreted with caution, as recent research did not replicate this effect (Cavillo \& Emami, 2019; van Schie \& Leer, 2019).

Given this potential side effect, the question arises how knowledgeable EMDR practitioners are about human memory. Practitioners' beliefs about how traumatic memory works has been an important topic in psychology over the past two decades (Loftus, 1993; Schacter, 1996). These beliefs were at the centre of a heated debate between researchers and clinicians about the accuracy of childhood sexual abuse memories that surface during psychotherapy (i.e., recovered memories), a debate also known as the memory wars (Crews, 1995). Some clinicians (e.g., Freyd, 1994) argued that individuals cope with traumatic experiences by blocking them out of consciousness into the unconscious (i.e., repression) or by dissociating (i.e., compartmentalization) them from consciousness. According to this view, repression or dissociation make traumatic memories temporarily inaccessible, but with the help of psychotherapy, these memories may re-emerge into patients' consciousness (Ceci \& Loftus, 1994). This view has its roots in the psychoanalytic theory of Freud and it proposed that repression operates unconsciously on threatening mental contents (Freud, 1910; however Freud also used the word repression to mean a conscious act, see Erdelyi, 2006). Whereas repression is usually considered an unconscious act, suppression is seen as a conscious act in which traumatic memories are forgotten due to conscious motivation to avoid such memories. It is believed that repressed memories of trauma will manifest 
themselves in the experience of mental and/or physical symptoms (Hornstein, 1992). To alleviate these symptoms, the unconscious trauma must become conscious again. This "body keeps the score" hypothesis states that a narrative of the trauma is not formed, because the trauma is saved on an implicit level (i.e., in the body; van der Kolk \& Fisler, 1995). Others argued that there is little evidence for repression (or dissociative amnesia; see Otgaar, Howe, Patihis et al., 2019). Research generally indicates that traumatic memories are often well retained and easily retrievable (e.g., McNally, 2003). Many researchers also emphasized that certain therapeutic techniques (e.g., imagination, dream interpretation) might lead to the production of false memories, thereby referring to lab studies in which participants created false memories due to misinformation (Loftus, 1993; 2005).

The debate about repressed or dissociated memories inspired surveys that tried to gauge what clinicians think about human memory. One of the earliest examples is the study of Yapko (1994), who found that around 516 therapists (60\%) indicated a belief in the existence of repressed memories (see also Dammeyer, Nunez Nightingale, \& McCoy, 1997; Golding, Sanchez, \& Sego, 1996). Recent surveys suggest that some therapists continue to hold controversial beliefs about memory. Ost and colleagues (2013) conducted an online survey among chartered clinical psychologists and hypnotherapists. Overall, 66 respondents $(27.8 \%)$ indicated they had seen a patient with a recovered memory in their clinical setting. Fifty-three respondents (22.5\%) thought that such reports are usually or always accurate. Seventy-two respondents (32.4\%) indicated they had treated a patient who reported satanic/ ritualistic abuse. Eighty respondents (38.2\%) believed that such reports could usually or always be seen as accurate. Interestingly, 53 respondents (34.6\%) also said they had seen cases of patients with potential false memories. Thus, this survey suggests that at least a minority of therapists still hold beliefs about memory that are controversial.

Patihis and colleagues (2014; Study 1) surveyed undergraduate students' beliefs about memory. Participants responded to each memory statement (e.g., "traumatic memories are often repressed") on a 6 -point scale ( 1 = strongly disagree; 6 = strongly agree). A large proportion ( $n=316,81 \%$ ) of students "slightly agreed" with this statement, thus expressing at least some belief in the existence of repressed memories. In their second study, the authors conducted an online survey among researchers, clinicians, undergraduate students, and the general public. Thirty-five clinicians (60.3\%) agreed that traumatic memories can be repressed compared with 12 researchers (19.4\%). In addition, 25 clinicians (43.1\%) believed that repressed memories can be retrieved during therapy compared to ten researchers 


\section{Chapter 4}

(16.1\%). These findings should be interpreted with caution, because the way survey items are formulated might lead to inflated proportions of controversial memory beliefs (see for a discussion Brewin et al., 2019; Otgaar, Wang et al., 2019). Still, controversial beliefs that are held firmly by clinicians (e.g., believing in repressed memories without reservation) may be problematic in a treatment setting (Lilienfeld, Lynn, \& Beyerstein, 2010). Specifically, such beliefs could spawn clinicians to develop a flawed treatment plan and/or use suggestive techniques leading to false memories in patients (Loftus, 1993). With this in mind, the current studies surveyed samples of EMDR practitioners, students, and researchers about their understanding of how memory operates. In doing so, we not only employed simple survey statements but also a case vignette. In Study 1, we attempted to examine memory beliefs in a small number of EMDR practitioners and students. In Study 2, we explored beliefs in a larger sample, which included students, EMDR practitioners, and researchers.

\section{Study 1}

\section{METHOD}

\section{Participants}

We recruited 47 participants: 35 Master's students and 12 EMDR practitioners. The student subsample consisted of students in a clinically oriented Master's program at Maastricht University $\left(M_{\text {age }}=23.97, S D=2.90\right.$, range $21-32,34$ women). These students are taught about various mental healthcare issues, they gain practical skills necessary to manage such issues, and are introduced to basic knowledge of EMDR (i.e., EMDR as a treatment option for PTSD). They had no practical experience with EMDR. They were recruited before the start of a tutorial meeting. Seventeen EMDR practitioners were recruited via the chair of a special interest group or word of mouth. Five EMDR practitioners failed to complete the survey. They did not differ on demographic variables from practitioners who did complete the survey. Hence, 12 EMDR practitioners were included $\left(M_{\text {age }}=44.33, S D=9.26\right.$, range 29 - 56, all 12 were women). The study was approved by the standing ethical committee of the Faculty of Psychology and Neuroscience, Maastricht University.

\section{Materials and Procedure}

All anonymous data and materials are available at the Open Science Framework at https:// 
osf.io/4ug9t. The survey took about 20 minutes to complete and was conducted on paper (students) or online at a time and place of participants' choosing (EMDR practitioners).

Case Vignette. Participants were presented with a case vignette of a 29-year-old patient (https://osf.io/t9d4j). The patient experienced a range of symptoms that are also stipulated in the Diagnostic and Statistical Manual of Mental Disorders (fifth edition; Americam Psychiatric Association, 2013) criteria for PTSD (e.g., anxiety, nightmares, difficulty sleeping). However, the patient had no specific trauma memory at the beginning of therapy. After one EMDR therapy session targeting a childhood memory of being abandoned by her mother, the patient recovered a memory of childhood sexual abuse. The patient attributed the complaints and symptoms to this memory. After several EMDR sessions, the treatment ended. Participants indicated how likely it is that the patient described a memory of an authentic event on a 4-point scale ( 1 = very likely; 2 = likely; 3 = unlikely; 4 = very unlikely). Participants could elaborate their answer.

Memory Beliefs Questionnaire. The Memory Beliefs Questionnaire was based on earlier work (derived from Ost et al., 2013, and Patihis et al., 2014; https://osf.io/vaq35) and consisted of 15 Dutch statements about the functioning of memory. The questionnaire included two correct statements (e.g., "memory can be inaccurate") and 13 controversial statements (e.g., "memory is not influenced by suggestion"; "repressed memories of events that did happen can be retrieved in therapy accurately"). Participants indicated to what extent they agreed with the statements on a 4-point scale ( 1 =totally disagree; 2 = disagree; 3 = agree; 4 = totally agree). In line with Ost et al. (2013) and Patihis et al. (2014), a "do not know" option was not provided. Participants were also asked to rate how often they read technical literature on memory on a 3-point scale (1 = below average: e.g., I rarely read scientific articles about memory; 2 = average: e.g., l occasionally read journal articles about memory; 3 = above average: e.g., I regularly read a scientific article about memory).

Therapy Experience Questionnaire. The therapy questionnaire (https://osf.io/qygnf) consisted of 14 questions that were derived from Ost and colleagues (2013). The questions covered topics on, for example, vague memories during a therapy session, unexpected events during a therapy session, and sexual/ritualistic abuse (e.g., "how often have you seen spontaneous memories of trauma were revealed by the patient during a therapy session?"). The EMDR practitioners completed the therapy questionnaire and they indicated on a 5-point scale ( 1 = never; 2 = rarely; 3 = sometimes; 4 = most of the time; 5 = always) how often over the last 15 years they had encountered such events in their practice. The EMDR practitioners 


\section{Chapter 4}

could elaborate upon their answer. As the sample size was limited, we do not address the results here, but they can be found on the OSF (https://osf.io/3prg4).

\section{RESULTS}

Due to the small sample size and constraints on generality, no inferential statistics are reported. Confidence intervals are reported to represent population estimates.

Case Vignette. Participants who chose likely or very likely were counted as finding the memory likely to be authentic. Twenty-one students $(60.0 \%, 95 \% \mathrm{Cl}[42.2-75.7 \%])$ and nine EMDR practitioners $(75.0 \%, 95 \% \mathrm{Cl}[42.8-93.3 \%])$ indicated that the recovered memory of the patient was (very) likely to be authentic. For elaborations, see OSF (https://osf.io/3prg4).

Memory Beliefs Questionnaire. Participants who chose agree or strongly agree were counted as agreeing with a statement, but see Table $\mathbf{4 . 1}$ for an overview including all answer categories. EMDR practitioners ( $n=12,100 \%, 95 \% \mathrm{Cl}[69.9-100 \%])$ agreed more often than students ( $n=25,71.4 \%, 95 \% \mathrm{Cl}[53.5-84.8 \%])$ with the statement that memory can be inaccurate. Students $(n=20,57.1 \%, 95 \% \mathrm{Cl}[39.5-73.2 \%])$ agreed more often than EMDR practitioners $(n=3,27.3 \%, 95 \% \mathrm{Cl}[7.3-60.7 \%])$ that suggestibility is a problem for young children. A majority of students ( $n=32,91.4 \%, 95 \% \mathrm{Cl}[75.8-97.8 \%])$ and EMDR practitioners $(n=11$, $91.7 \%, 95 \% \mathrm{Cl}$ [59.8 - 99.6\%]) agreed that an individual may develop false memories for nontraumatic events. A minority of students and EMDR practitioners agreed with the statements that memory is like a video camera $(n=1,2.9 \%, 95 \% \mathrm{Cl}[0.15-16.6 \%]$ and $n=1,8.3 \%, 95 \%$ $\mathrm{Cl}[0.4-40.2 \%]$ respectively), that early memories are accurately stored $(n=2,5.7 \%, 95 \% \mathrm{Cl}$ [1.0 - 20.5\%] and $n=0,0.0 \%, 95 \% \mathrm{Cl}[0.0-30.1 \%]$ respectively), and that a poor memory of childhood events is indicative of a traumatic childhood $(n=7,20.0 \%, 95 \% \mathrm{Cl}[9.1-37.5 \%]$ and $n=2,16.7 \%, 95 \% \mathrm{Cl}$ [2.9-49.1\%] respectively).

A large majority of students and EMDR practitioners agreed with the controversial statement that the mind is capable of unconsciously blocking out memories of traumatic events $(n=31,88.6 \%, 95 \% \mathrm{Cl}[72.3-96.3 \%]$ and $n=11,91.7 \%, 95 \% \mathrm{Cl}[59.8-99.6 \%]$ respectively), and that repressed memories of events can be accurately retrieved in therapy ( $n=31,88.6 \% 95 \% \mathrm{Cl}$ [72.3 - 96.3\%] and $n=11,91.7 \%, 95 \% \mathrm{Cl}$ [59.8 - 99.6\%] respectively). Furthermore, 17 students (48.6\%, 95\% Cl [31.7 - 65.7\%]) and four EMDR practitioners (33.3\%, 
95\% $\mathrm{Cl}[11.3-64.6 \%])$ agreed that very vivid memories are more likely to be accurate than vague memories. Nontrivial proportions of students and EMDR practitioners also agreed with the controversial idea that hypnosis can accurately retrieve inaccessible memories of events that did happen $(n=25,71.4 \%, 95 \% \mathrm{Cl}[53.5-84.8 \%]$ and $n=7,58.3 \%, 95 \% \mathrm{Cl}[28.6-83.5 \%]$ respectively). About half of the students ( $n=20,57.1 \%, 95 \% \mathrm{Cl}[39.5-73.2 \%])$ and EMDR practitioners $(n=6,50.0 \%, 95 \% \mathrm{Cl}[22.3-77.7 \%])$ believed in the existence of photographic memory.

\section{DISCUSSION}

Our samples of EMDR practitioners and students rarely endorsed scientifically unsupported statements (e.g., "early memories are accurately stored"). Nevertheless, a majority in both groups seemed to believe in repressed memories. In addition, a majority in both groups indicated that the recovered memory of the vignette was likely to be authentic.

Study 1 suffered from three limitations. First, the sample size of EMDR practitioners was small. Second, in line with previous studies (Akhtar, Justice, Knott, Kibowski, \& Conway, 2018; Ost et al., 2013; Patihis et al., 2014) a "do not know" option was not provided. This might have restricted respondents' option to indicate when they had no opinion on a certain statement. The consequence is that respondents are forced to answer, when in fact they would be willing to admit that they have no knowledge concerning a particular statement (see also Brewin et al., 2019). Third, for some concepts (e.g., repressed memories), no additional explanation was provided in the questionnaire. This is problematic as participants may have their own interpretations on what, for example, repressed memory entails and this might not be in line with how the concept is viewed within the survey (McNally, 2016; Brewin et al., 2019). Study 2 relied on a larger sample of EMDR practitioners. In the questionnaire, a "no opinion" option was included and concepts (e.g., repression) were not explicitly mentioned. 


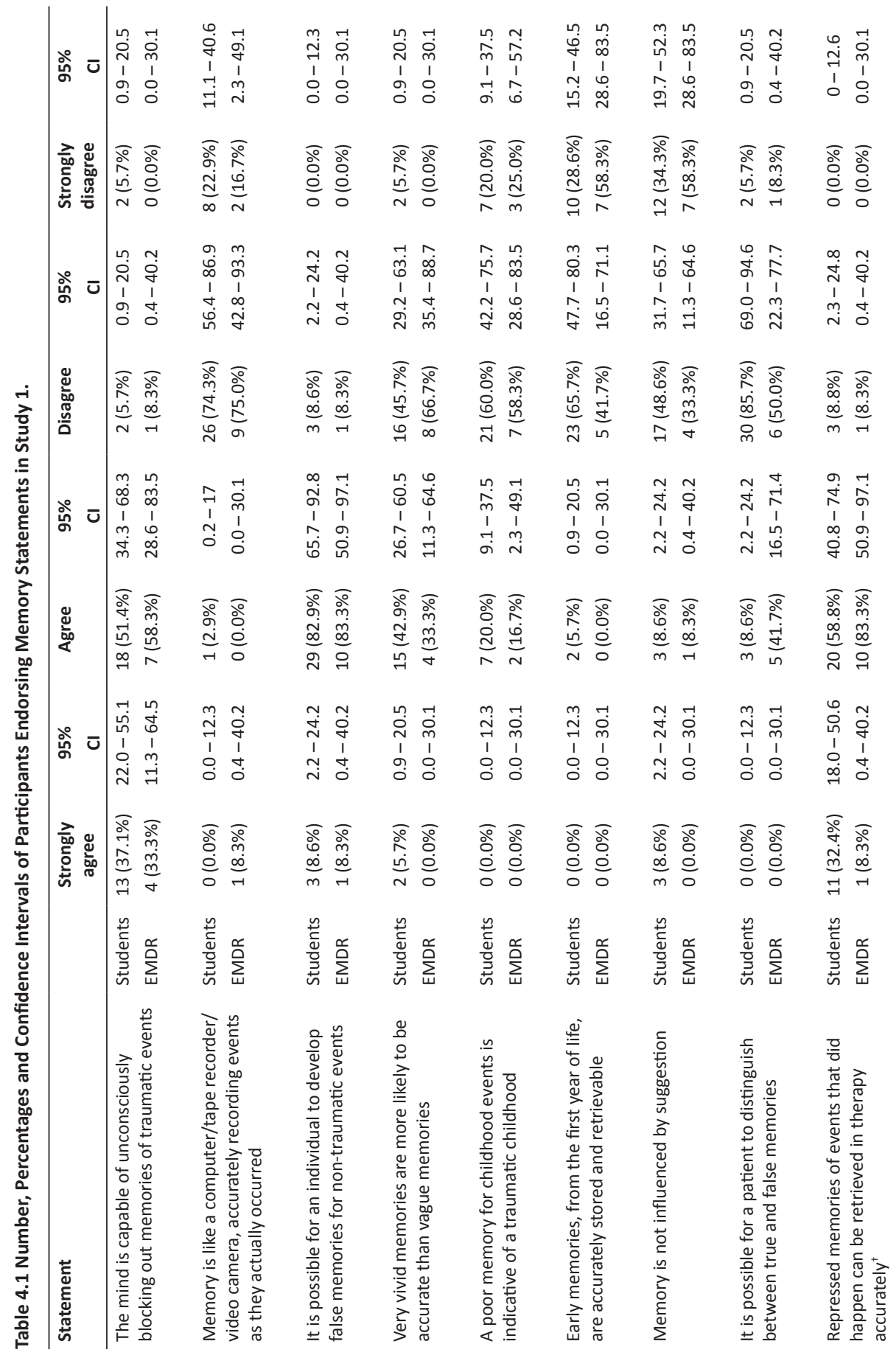




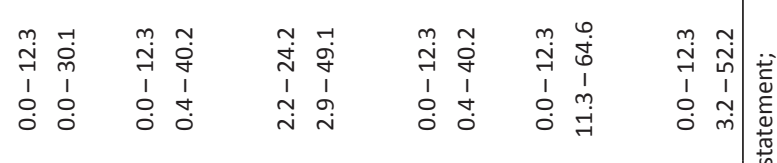

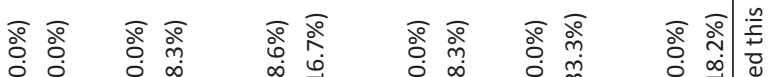

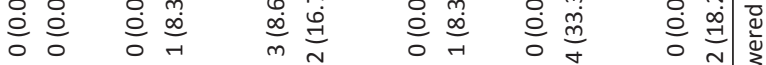

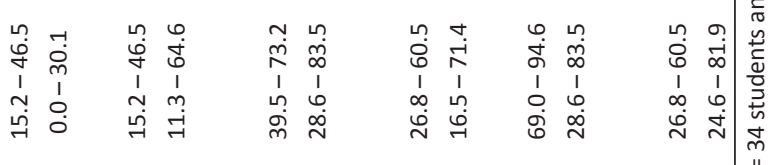

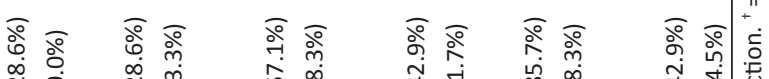

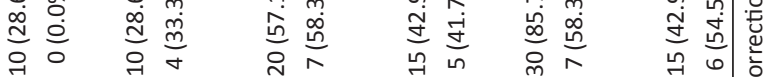

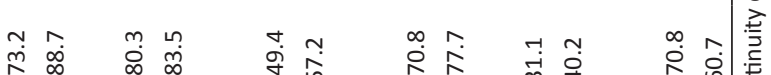

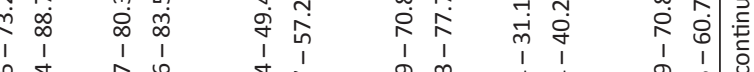

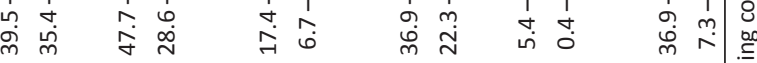

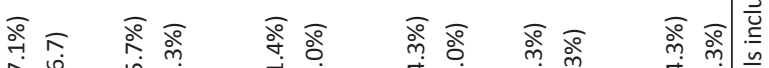

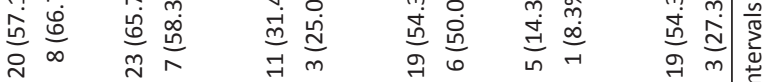

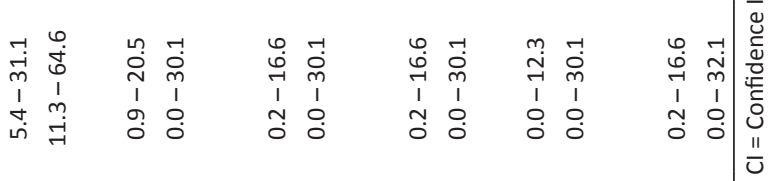

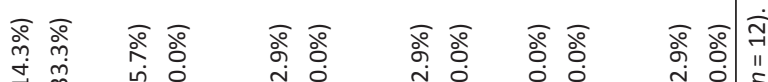

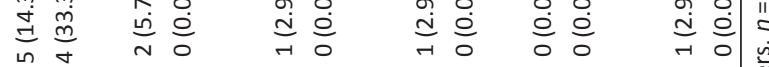

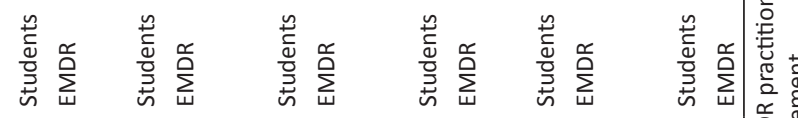

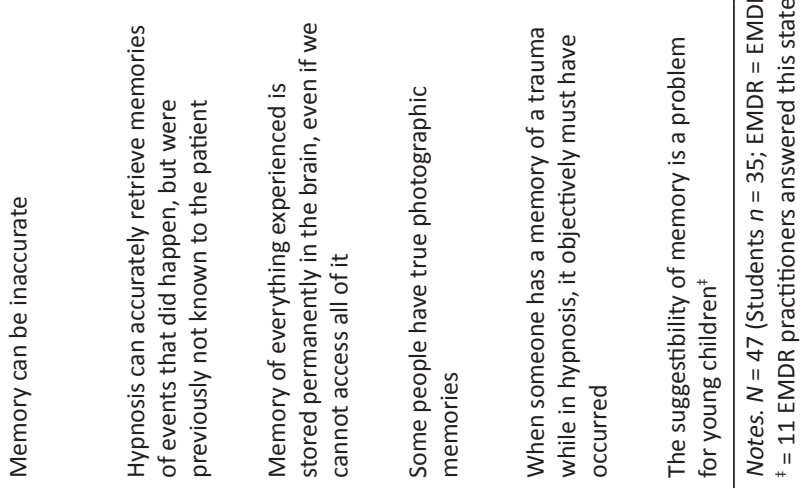




\section{Study 2}

\section{METHOD}

\section{Participants}

Ninety-five participants were recruited for the current study. The sample consisted of 24 students in a clinically forensic oriented Master's program at the University of Groningen $\left(M_{\text {age }}=23.75, S D=3.01\right.$, range $20-35,21$ women). These Master's students all had one week of information on PTSD and EMDR. During courses, EMDR was presented as a therapeutic intervention. The students were recruited within the context of a lecture. Forty-five EMDR practitioners were recruited on a training day organized by their mental health care facility. Four EMDR practitioners did not provide consent to use their answers for scientific purposes (see below), hence, 41 EMDR practitioners ( $M_{\text {age }}=40.39, S D=11.18$, range $22-61,33$ women) were included. Thirty-five academics (including PhD students, post-docs and lecturers/ professors, no adjuncts were approached) with a research appointment at a clinically oriented department of the University of Groningen (hereafter: "researchers") were recruited during a lab meeting. Five researchers did not provide their consent, hence, 30 academics $\left(M_{\text {age }}=\right.$ 31.14, SD = 6.64, range 22 - 45, 25 women; mostly junior researchers, such as PhD students and post-docs) were included. Half of the researchers had some clinical experience (e.g., internship of two years), but they were not experienced enough to be counted as EMDR practitioners. The standing ethical committee of the Psychology department of the University of Groningen approved the study.

\section{Materials and Procedure}

All anonymous data and materials are available at the Open Science Framework at https:// osf.io/4ug9t. The survey took about 15 minutes to complete online, before participants' respective meetings. Prospective participants received the link to the questionnaire by email and completed it at their own convenience. The researchers and students completed an English version and the EMDR practitioners completed the questionnaire in Dutch. They were asked to give their opinion on statements about memory as input for a talk/lecture on memory, and students filled out the questionnaire before reading the relevant literature on the topic. Afterwards, participants were asked to provide their consent to use their answers for scientific purposes. The "agree" option contained an explanation of participants' rights 
and it was stated that additional questions about the participants' background would follow (e.g., biographical and educational information). The "disagree" option stated that choosing this option would terminate the questionnaire without any consequences. Respondents were assured that in that case, their answers would only be used on a group level in the talk/ lecture for purpose of demonstration. After reading the information, prospective participants were given the choice either to participate and carry on or to decline and terminate the questionnaire.

Statements about Memory. The questionnaire (https://osf.io/5kc72) was constructed using Qualtrics software, Version December, 2017. It consisted of 18 statements about memory and was in part inspired by those used in previous studies (Magnussen \& Melinder, 2011; Ost et al., 2013; Odinot et al., 2015). New statements on bodily memories and preverbal trauma were constructed using various sources (e.g., Went, 2016; http://www.larrysroadmap.com/ memory/body-memory). The questionnaire included six correct statements (e.g., "memory of trauma can be influenced by suggestion"), six scientifically controversial statements (e.g., the human mind is able to unconsciously block access to traumatic memories), and six statements for which empirical evidence is lacking or indecisive (e.g., "it is possible that traumatic memories are stored in the body"). Participants had to indicate their opinion ( 1 = agree; 2 = disagree; 3 = no opinion) and the statements were randomly presented. This questionnaire differed from the Memory Beliefs Questionnaire used in Study 1 in the following ways: it contained more topics (e.g., statements on preverbal trauma that are relevant for EMDR practitioners) and statements were formulated by describing the meaning of a concept rather than using the label.

\section{RESULTS}

Due to the small sample size and constraints on generality, no inferential statistics are reported. Confidence intervals are reported to represent population estimates. Table $\mathbf{4 . 2}$ gives an overview of all scores and effect sizes. Here, we will highlight some of the most important results reported in Table 4.2.

We found that 22 students $(91.7 \%, 95 \% \mathrm{Cl}$ [71.5 - 98.5\%]), 38 EMDR practitioners $(92.7 \%, 95 \% \mathrm{Cl}[79.0-98.1 \%])$, and 29 researchers $(96.7 \%, 95 \% \mathrm{Cl}[81.0-99.3 \%])$ agreed that even very vivid memories can be false. Twenty-one students $(87.5 \%, 95 \% \mathrm{Cl}[66.5-$ 96.7\%]), 40 EMDR practitioners (97.6\%, 95\% Cl [85.6 - 99.9\%]), and 29 researchers (96.7\%, $95 \% \mathrm{Cl}[81.0-99.3 \%])$ agreed that memory cannot be compared to a video recording and 


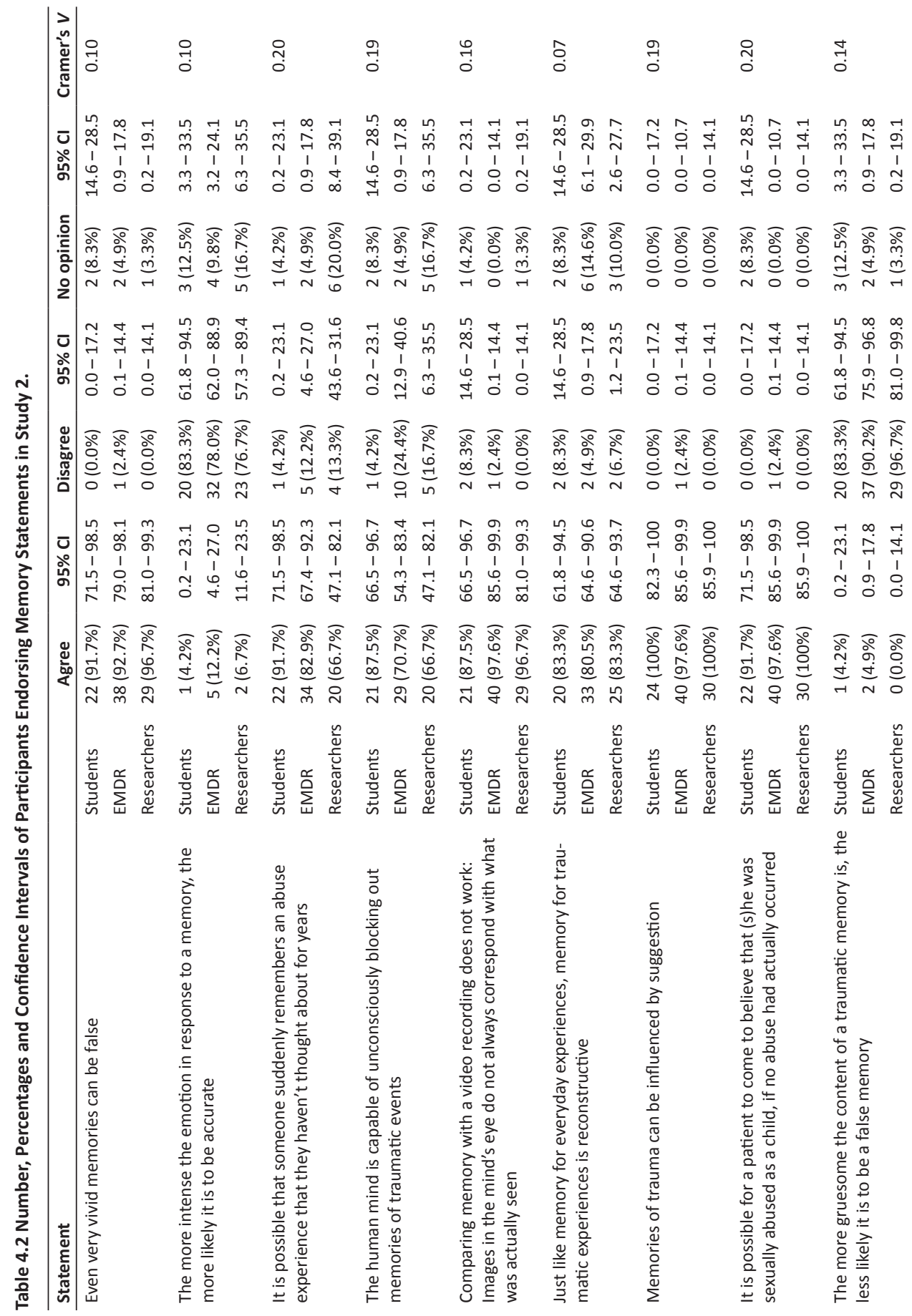




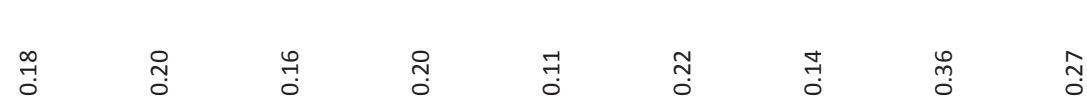

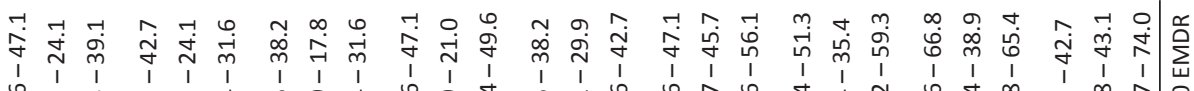

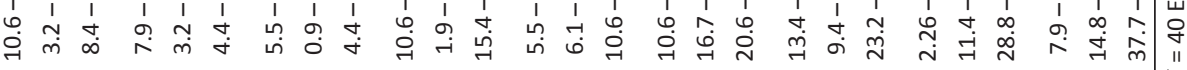

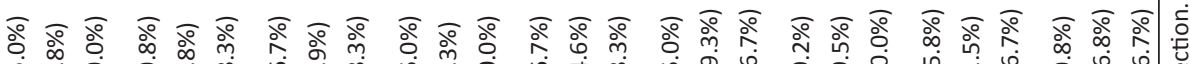
岂

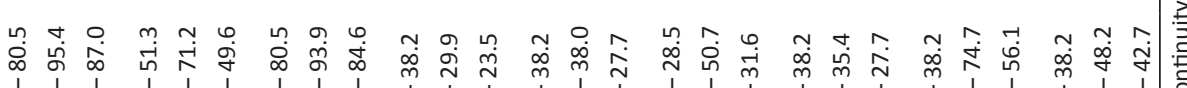

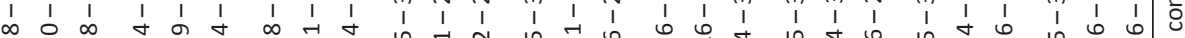

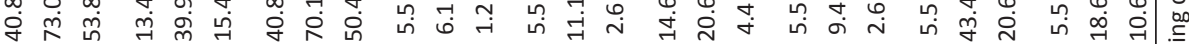

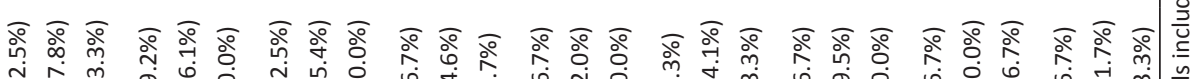
它

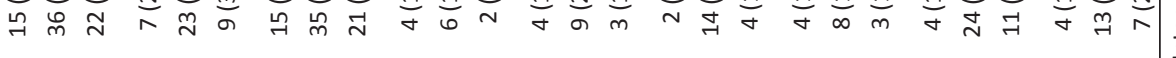

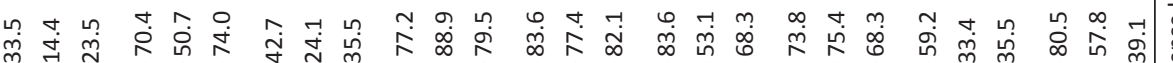

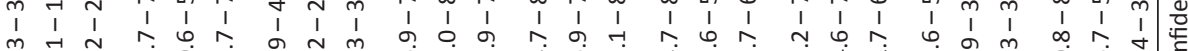
m

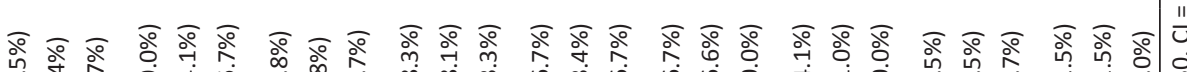

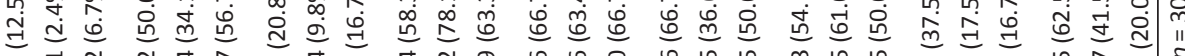

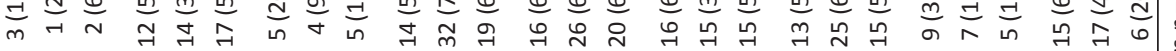

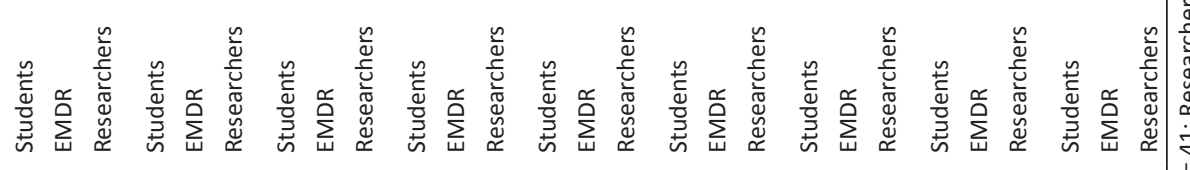

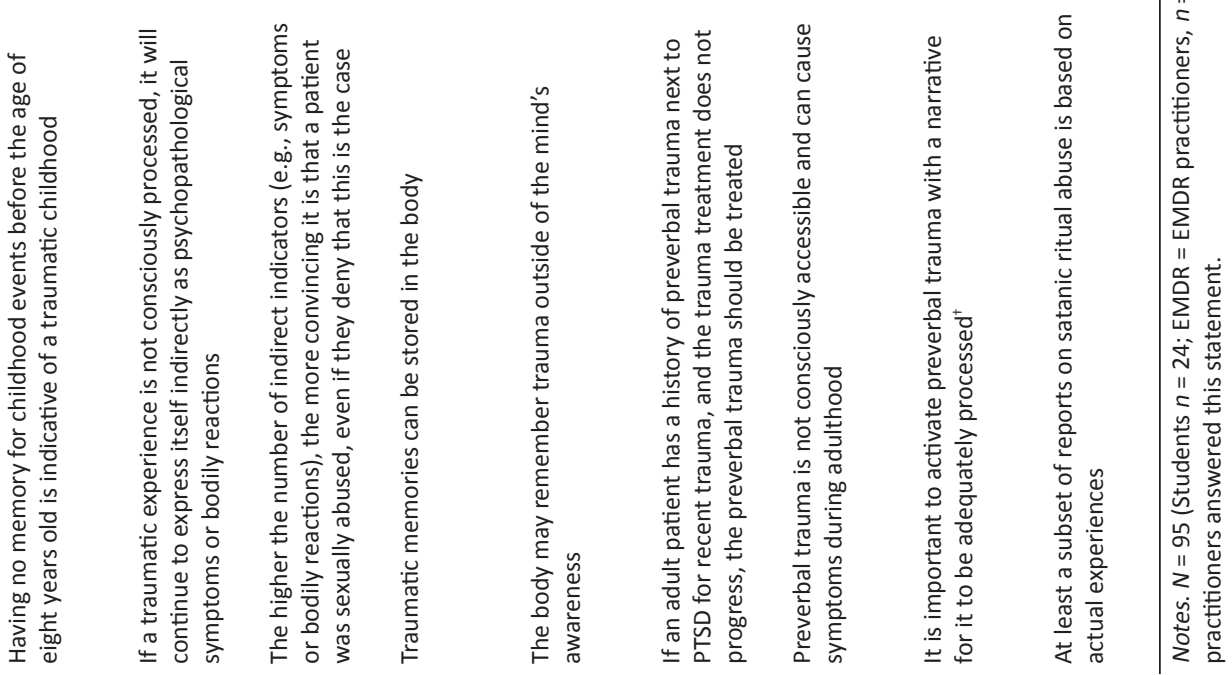




\section{Chapter 4}

that the images in our mind do not always correspond with what was actually seen. Twenty students $(83.3 \%, 95 \% \mathrm{Cl}[61.8$ - 94.5\%]), 33 EMDR practitioners $(80.5 \%, 95 \% \mathrm{Cl}[64.6-$ $90.6 \%])$, and 25 researchers $(83.3 \%, 95 \% \mathrm{Cl}[64.6-93.7 \%])$ also agreed that memory for traumatic experiences is reconstructive.

A majority of participants within all groups also believed that it is possible for a patient to become convinced that he/she was sexually abused as a child, when in reality this did not happen ( $n=22$ (91.7\%, 95\% Cl [71.5 - 98.5\%]), $n=40$ (97.6\%, 95\% Cl [85.6 - 99.9\%]), and $n=30(100 \%, 95 \% \mathrm{Cl}[85.9-100 \%])$, respectively). Twenty-two students $(91.7 \%, 95 \%$ $\mathrm{Cl}[71.5$ - 98.5\%]) and 34 EMDR practitioners (82.9\%, 95\% Cl [67.4 - 92.3\%]) agreed that it is possible for an individual to suddenly remember an abuse experience while not having thought about it for years, whereas 20 researchers $(66.7 \%, 95 \% \mathrm{Cl}$ [47.1 - 82.1\%]) agreed. On the other hand, 21 students $(87.5 \%, 95 \% \mathrm{Cl}$ [66.5 - 96.7\%]), 29 EMDR practitioners (70.7\%, $95 \% \mathrm{Cl}[54.3-83.4 \%])$ and 20 researchers $(66.7 \%, 95 \% \mathrm{Cl}$ [47.1 - 82.1\%] agreed that the human mind is capable of unconsciously blocking out memories of traumatic events.

There was little consensus with regard to topics such as body memories and preverbal trauma. For example, 12 students $(50.0 \%$, 95\% Cl [29.7 - 70.4\%]), 14 EMDR practitioners (34.1\%, 95\% Cl [20.6 - 510.7\%]) and 17 researchers (56.7\%, 95\% Cl [37.7 - 74.0\%]) agreed that if a traumatic experience is not consciously processed, it will continue to express itself indirectly as psychopathological symptoms or bodily reactions. Regarding statements on preverbal trauma, 16 students $(66.7 \%, 95 \% \mathrm{Cl}$ [44.7 - 83.6\%]), 15 EMDR practitioners (36.6\%, $95 \% \mathrm{Cl}[22.6$ - 53.1\%]) and 15 researchers (50.0\%, 95\% Cl [31.7 - 68.3\%]) agreed that when an adult patient in addition to PTSD has a history of preverbal trauma, the preverbal trauma must be treated.

\section{DISCUSSION}

Many students, EMDR practitioners, and researchers in our sample had a nuanced view on several issues about memory. For example, many endorsed ideas that very vivid memories can be incorrect, that trauma memories are reconstructive, and that memory does not operate as a video camera. A majority of all groups believed that an individual could suddenly remember or become convinced of an abusive experience. However, we still observed considerable proportions of students (87\%) and EMDR practitioners (70\%) who agreed with controversial claims about the existence of repression-like phenomena. A somewhat lower 
level of endorsement was found for researchers (around 66\%). Little consensus in these groups was found regarding topics such as the body keeps the score, and preverbal trauma. This could be due to the fact that empirical evidence is either lacking or weak. To conclude, Study 2 replicated the findings of Study 1 and indicated that scepticism and problematic ideas about memory may co-exist within one group.

\section{GENERAL DISCUSSION}

In the 1990s, creating false memories during therapy has been the topic of an intense debate. At the heart of this debate was the question whether memories that surface during or after therapeutic instructions are veridical. Clinicians believed that traumatic memories were blocked out of consciousness into the unconscious. By means of psychotherapy, such memories could re-emerge into patients' consciousness. However, scientific findings do not prove the existence of repression and allocate the emergence of memories to suggestive therapeutic instructions (Ceci \& Bruck, 1993).

Because EMDR addresses traumatic autobiographical memories, it is important that EMDR practitioners are sensitive to controversial ideas about the functioning of human memory. We examined memory beliefs in samples of EMDR practitioners, because of the increasing popularity of EMDR as a therapeutic technique (Herbert et al., 2000; Gielkens, Sobczak, \& Van Alphen, 2016).

The most important findings can be summarized as follows. First, in both studies, students, a small sample of EMDR practitioners, and researchers demonstrated adequate knowledge on various memory-related issues (see also Brewin et al., 2019). That these groups were generally reluctant to endorse some controversial beliefs about memories is encouraging and shows that, to some extent, they are able to differentiate between wellsupported and problematic ideas about memory functioning. Nevertheless, we observed in our samples that many EMDR practitioners (70-90\%) and students (around 90\%) believed in the existence of repression. Similar percentages (around $80 \%$ ) were found among the general public (Patihis et al., 2014, Study 2). A majority of the researchers (Study 2; 66.7\%) also agreed with repression statements, although they agreed to a lesser extent to this than students did. Hence, in one and the same group, scepticism about problematic memory notions may co-exist with endorsement of other controversial ideas about memory-related topics. In addition, in Study 2 we included statements referring to "the body keeps the score" 


\section{Chapter 4}

hypothesis (van der Kolk \& Fisler, 1995). During the memory wars, it was believed that the unconsciously blocked memory would express itself as mental and/or physical symptoms. Though empirical evidence for this is weak or absent (Vervaeke, Bogaerts, \& Heylen, 2002), only a minority of participants disagreed or had no opinion about such statements. Hence, a proportion of our sample beliefs that a traumatic memory must become conscious to treat physical symptoms that, allegedly, occur because of the traumatic memory.

Second, memory attitudes were similar in this sample of EMDR practitioners and researchers. This seems not to be in line with the findings of Patihis and colleagues (2014), who observed a practitioner-researcher gap in their study. However, it is vital to acknowledge that the subsample of researchers in Study 2 were research experts in the field of cognitive/ clinical psychology and not necessarily experts in the field of memory. Memory experts are generally sceptical towards the existence of repression (Patihis, Ho, Loftus, \& Herrera, 2018).

Third, memory attitudes of students and this sample of EMDR practitioners were, in many respects, similar. Although experience does not guarantee expertise (see e.g., Kahneman \& Klein, 2009), this raises the question of what role expertise plays in therapy (Tracey, Wampold, Lichtenberg, \& Goodyear, 2014). Given their expertise, one would have expected lower levels of endorsement of controversial memory ideas in practitioners. However, the question remains whether therapists act upon their knowledge regarding memory findings. More specifically, Gore-Felton and colleagues (2000) have shown that therapists' personal beliefs influenced their professional judgments. This might be problematic when a therapist personally beliefs in the concept of repression and clinical judgments are based on this belief.

If these results would generalize to EMDR therapists in general, then some of our results might be seen as worrisome for clinical settings. Believing in the concept of repression might fuel the idea that having vague symptoms or suspicions of sexual abuse might be a sign of a repressed memory, something that is highly controversial in memory literature (Rofé, 2008). In the worst-case scenario, therapists might suggestively search for the existence of repressed memories, thereby increasing the likelihood of inducing a false memories and/ or beliefs during a therapy session. In practice, if an EMDR practitioner decides to opt for EMDR when the patient has a vague memory, the patient could form new images because of subtle suggestions by a therapist and may conclude that the alleged event might have happened. Such false memories might be devastating when they go beyond the therapeutic context and affect relationships and/or enter the legal arena. Precisely because there is no general false memory trait (Patihis, 2018), clinicians should be aware that every patient could 
be susceptible to false memories (Bernstein et al., 2018; Patihis, Frenda, \& Loftus, 2018). Therefore, it is imperative for EMDR practitioners to refrain from suggesting the possibility of repressed memories of abuse to their patients, particularly in cases where patients have vague memories (de Jongh \& Wessel, 2018).

Patihis and Pendergrast (2019) found that some of the U.S. public who reported undergoing EMDR indicated that their therapist discussed repressed and recovered memories with them (Tables S6 and S7; see Patihis \& Pendergrast, 2019). This is reinforced by previous related work that showed beliefs about repression are common (e.g., Ost et al., 2013; Patihis et al., 2014). A side note is that concepts such as repression are engrained in our culture and are casually used without being aware of possible implications (Lilienfeld, Lynn, \& Beyerstein, 2010). As a result, it remains difficult to capture the belief in repression in single statements.

The present studies have some limitations. Although the EMDR sample was small, our sample of EMDR are members of a specialized facility and the Dutch association of EMDR (around 4000 members in April, 20194) and work at a specialized facility in which EMDR is a core activity. Hence, the question remains whether the results based on this sample of EMDR practitioners can be generalized to the majority of Dutch EMDR practitioners. Our results are certainly not generalizable to EMDR practitioners around the world. Also, the sample in Study 2 consisted mainly of junior researchers (i.e., PhD students), and these results might not be generalizable to all (senior) academics of other universities. A direct replication should include a larger sample in all participants groups. However, we have no reason to believe that our results depend on other characteristics of the participants, materials, or context. In addition, in both Study 1 and 2, although a question assessing reading of the literature was included, the memory belief items did not include a question assessing the participants' self-assessed knowledge of the topic. Such self-assessments of knowledge would be an interesting addition to a future study.

EMDR decreases the vividness and emotionality of negative autobiographical memories (but see van Schie et al., 2019), but its long-term clinical value needs to be determined. However, our findings underscore the need for specialized education of clinicians about the functioning of memory and potential side effects of therapies that rely on changing the quality of autobiographical memories. In addition, it is important that memory scholars and clinicians continue to talk about these issues in academic and clinical settings in order to prevent false memory production in therapy, as happened in the past. Such recovery of purportedly repressed memories could perilous for both patients who have to deal with traumatic 


\section{Chapter 4}

experiences and for individuals who start to believe to have experienced a traumatic event.

As EMDR therapists strongly believe in the concept of repression, it is likely that they (unintentionally) provide suggestive information or ask suggestive questions to their patients. To examine whether misinformation affects one's memory, the next chapter will discuss an experimental study that examines the link between eye movements and suggestive false memories. 



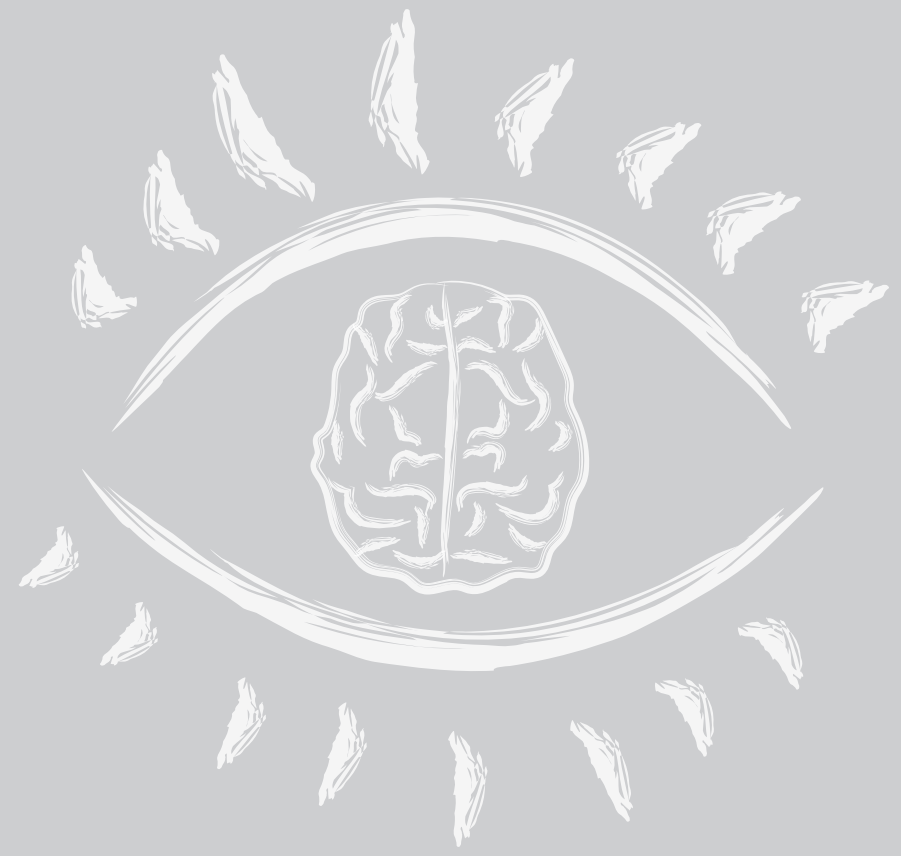




\section{CHAPTER 5}

\section{Eye movements and suggestion-induced false memories}

Published as:

Houben, S. T. L., Otgaar, H., Roelofs, J., \& Merckelbach, H. (2018). Lateral eye movements increase false memory rates. Clinical Psychological Science, 6(4), 610-616. https://doi.org/10.1177/2167702618757658 



\section{INTRODUCTION}

Eye Movement Desensitization and Reprocessing (EMDR; Shapiro, 1989) is currently one of the most frequently used interventions to treat individuals with mental disorders, such as post-traumatic stress disorder (PTSD; van den Hout \& Engelhard, 2012). The crux of EMDR is that a client is instructed to perform eye movements while retrieving a traumatic event. The purpose of these eye movements is to reduce the vividness and emotionality linked to the traumatic memory. Despite its popularity and the numerous studies examining symptom reduction after EMDR, little is known about the potential drawbacks of eye movements (Lilienfeld, 2007). We examined one potential drawback. Specifically, we tested whether eye movements might enhance the susceptibility to misinformation.

Experimental work has confirmed that performing eye movements significantly reduces the vividness and emotionality of traumatic memories (Lee \& Cuijpers, 2013). One explanation for this is that performing eye movements while focussing on the most salient aspects of a traumatic memory requires working memory capacity and performing this dual task interferes with the capacity for memory recall (van den Hout \& Engelhard, 2012). As a result, both vividness and emotionality of the memory decline. The rationale for this decline is the following. When imagining an event, a phenomenon called imagination inflation emerges, and the subjective confidence that the imagined event had occurred (e.g., by vivid images) increases (Goff \& Roediger, 1998; Otgaar, Scoboria, Howe, Moldoveanu, \& Smeets, 2016). The reverse is said to occur during eye movements. Eye movements make the memory less vivid, thereby making them less distinctive (i.e., imagination deflation, van den Hout \& Engelhard, 2012).

Before and after an EMDR session, therapists frequently engage in a comprehensive conversation (e.g., asking follow-up questions) to clarify certain aspects that surfaced during the session (de Jongh \& ten Broeke, 2016). Oftentimes, therapists have poor knowledge about issues related to memory functioning compared to what we know from the science of memory (Patihis et al., 2014). Given this state of affairs, follow-up questions during a therapeutic interview might unintentionally have a misinformation potential. Hence, it is surprising that little research has focused on whether eye movements might enhance susceptibility to misinformation (see Devilly \& Brown, 2011). This will be the focus of the current study.

The misinformation paradigm (Loftus et al., 1978) is a well-established procedure in which participants first witness an event (e.g., mock crime) and then receive suggestive 


\section{Chapter 5}

misinformation about the event (e.g., the robber used a knife, while no knife was present). Next, participants are interviewed about the details of the event. Research has consistently shown that exposure to misinformation can make participants include that misinformation as part of their own memory reports (i.e., reporting misinformation and thus creating false memories), an effect that has been termed the misinformation effect (Loftus, 2005). In the study of Parker and colleagues (2009), participants had to listen to a story and viewed pictures simultaneously that illustrated the narrative. Participants received misinformation by means of a post-event misinformation questionnaire. Hereafter, they were randomly assigned to a horizontal eye movement, vertical eye movement, or a control condition. Participants in the eye movement conditions were more resistant to the misinformation effect than the controls. However, this study cannot be applied to situations in which clients are first engaged in eye movements and receive misinformation afterwards.

Theoretically, participants might be most susceptible to misinformation when their memory of an event has become vague. In such a situation, it is difficult to determine the source of misinformation and so the misinformation might be experienced as part of the original record. The Source Monitoring Framework (SMF; Johnson et al., 1993) explains the misinformation effect as a failure to accurately identify the source of information. Divided attention can compromise this source attribution. Of relevance to the current experiment is research showing that divided attention can increase the probability of false memories (Otgaar, Peters, \& Howe, 2012). Performing eye movements and simultaneously retrieving a memory can be seen as a dual task where both tasks tax attentional resources. Our predictions are in line with the tenets of several false memory theories, such as fuzzy trace theory (FTT; Brainerd et al., 2008). FTT postulates that memories are stored along two independent memory traces: verbatim (i.e., item-specific characteristics) and gist (i.e., meaning of the information) traces. False memories arise due to reliance on gist traces, when verbatim traces are unable to be retrieved. With respect to the current experiment, this could indicate the following. As previous research has shown that eye movements decrease the vividness (and emotionality) of a memory (Lee \& Cuijpers, 2013), this could imply that the memory becomes less detailed. If so, after eye movements, individuals will rely more on gist traces, thereby making them more prone to accept misinformation. Also, associative activation theory (AAT; Howe et al., 2009) posits that false memories are the result of spreading activation. Recent research has shown that when misinformation is associatively related to an experienced event, misinformation is easily accepted as being part of this event (Otgaar, 
Howe, Brackmann, \& Smeets, 2016). When eye movements make an experienced event less distinctive, misinformation might be more easily accepted as it is less likely to be seen as discrepant and not-related to the event.

We examined whether eye movements increase the susceptibility to the misinformation effect. After watching a car crash video and performing eye movements or not, participants received misinformation. In line with previous research (Lee \& Cuijpers, 2013), we hypothesized that performing eye movements reduces the vividness and emotionality of the memory. Additionally, we expected that participants who had performed eye movements would show heightened levels of misinformation acceptance compared with control participants who did not engage in the eye movement task.

\section{METHOD}

\section{Participants}

Based on previous research (Parker \& Dagnall, 2007; study on eye movements and false memories with an effect size of $d=0.6$ ), an a priori analysis using G*Power (Faul et al., 2007), with a medium to large effect size $(d=0.6)$ and a power of 0.80 indicated a sample size of 72 participants. Ten extra participants were tested in case any drop outs took place. Thus, 82 second year undergraduate psychology students from Maastricht University $\left(M_{\text {age }}=21.42\right.$ years, $S D=2.08$, range 19-31; 54 women) were tested. Participants received course credits for their participation. The experiment was approved by the standing ethical committee for the Faculty of Psychology and Neuroscience, Maastricht University.

\section{Materials}

All material is available at the Open Science Framework at https://osf.io/j479p.

Beck Depression Inventory. The Beck Depression Inventory (BDI; Beck \& Steer, 1987) is a self-reported questionnaire that contains 21 items, including several statements of which participants have to select the statement that describes them best (e.g., item 1: "I do not feel sad - I feel sad - I am sad all the time and I cannot snap out of it - I am so sad and unhappy that I cannot stand $\left.i t^{\prime \prime}\right)$. The BDI was included purely for exploratory analyses. As EMDR is used for individuals who experienced a traumatic event, or are depressed as a result of this trauma (Shapiro \& Forrest, 2004), we want to explore whether participants with high BDI scores also show higher ratings on vividness and emotionality. 


\section{Chapter 5}

Video. Participants saw a video that has previously been used in the trauma film paradigm (TFP; Holmes \& Bourne, 2008; Strange \& Takarangi, 2012). It depicts a road accident where several cars crash into one another. Specifically, three women are in a car arguing about a text message that the driver is texting. As the driver does not pay attention to the road, they crash into another vehicle. As the car stops, another car crashes into the two vehicles. At least five people, including a baby, are injured and unconscious. Emergency services arrive and the video ends with a close up of the driver's face.

Eye Movement Task. To simulate the eye movement component of EMDR, we employed a computerized eye movement task that has been described in van den Hout and colleagues (2013). A grey dot was presented on a black background via E-Prime and moved from left to right with $1 \mathrm{~s}$ per cycle, during four intervals of $24 \mathrm{~s}$ with a $10 \mathrm{~s}$ interval. The stationary dot was presented via PowerPoint, also during four intervals of $24 \mathrm{~s}$ with a $10 \mathrm{~s}$ interval. This duration is in line with previous research (van den Hout \& Engelhard, 2012) and largely converges to what is usually done in EMDR therapy. All participants sat at a 30 $\mathrm{cm}$ distance from the computer screen. All participants were instructed to think about the video and the emotions that they felt during the video presentation. The researcher checked whether the participant complied with the instructions by monitoring the participant.

Misinformation. Misinformation was provided in the form of an eyewitness narrative and was presented on paper. The narrative contained ten true statements (e.g., "the girls were driving a small car") and five false statements about the video (e.g., "the driver was texting to John" instead of James; "I saw the injured mother" instead of father).

Recognition task. The recognition task contained 15 questions, including ten questions, with a true and foil answering option (e.g., "What vehicle were the girls driving?", true answer: a small car, foil answer: a van) and five critical questions pertaining to misinformation that was presented earlier (e.g., "To whom were the girls writing a text message?", true answer: James, misinformation answer: John). In line with previous research (Parker et al., 2009), the recognition test was presented orally to the participants.

\section{Design and Procedure}

The current experiment used a between-subjects design and included one independent variable with two levels: horizontal eye movements versus control (i.e., central fixation). The dependent variables were: (i) correct answers and (ii) endorsement of misinformation. All participants were tested individually in a quiet lab room. After obtaining written informed 
consent, participants completed the BDI. Then, they were told they would see a violent car crash. They were instructed to view the video carefully, as if they were an eyewitness. After the video had finished, they were asked to complete a visual analogue scale (VAS; Lee \& Cuijpers, 2013) for vividness and emotionality. The VAS ranged from (0) not vivid at all (10) extremely vivid for vividness and ranged from (0) extremely negative - (10) extremely positive for emotionality. From this point, participants were randomly assigned to either the eye movement (EM) condition or the control condition. EM participants were instructed to follow the moving dot on the computer screen with their eyes without moving their head, for four episodes of $24 \mathrm{~s}$. They were instructed to think about the video and the emotions that they felt during the video presentation. Control participants were instructed to watch the stationary dot for four episodes of $24 s$ while thinking about the video and the emotions that they felt during the video presentation. Immediately after the four episodes of $24 \mathrm{~s}$, participants were asked to rate the vividness and emotionality on a VAS again. Hereafter, participants had to complete a filler task (e.g., playing Bejeweled) for five minutes. Then, participants read a narrative from another eyewitness containing the misinformation. After completing another filler task for five minutes, and to end the session, participants received an oral recognition task. Participants were debriefed and received their course credit.

\section{RESULTS}

Vividness and Emotionality. Table $\mathbf{5 . 1}$ shows mean scores for vividness and emotionality. A repeated measures ANOVA for vividness revealed no significant interaction effect $(F(1,80)=$ $1.32, p=.26)$. No effect of Condition $(F(1,80)=1.025, p=.31$ ) was found. A significant effect of Time emerged $(F(1,80)=21.05, p<.001)$. Specifically, vividness scores significantly decreased within both conditions ( $t=4.84,95 \% \mathrm{Cl}[.50-1.21], p<.001$, Cohen's $d=.66$ for the EM condition, and $t=2.14,95 \% \mathrm{Cl}$ [.03-1.00], $p=.039$, Cohen's $d=.35$ for the control condition).

Emotionality scores increased from pre- to post-test in both conditions. A repeated measures ANOVA for emotionality revealed a nonsignificant interaction effect $(F(1,80)=3.79$, $p=.055)$. The effect of Condition was nonsignificant $(F(1,80)=0.94, p=.33)$. A significant effect of Time $(F(1,80)=8.53, p=.005)$ was found, showing that the video at post-test was experienced as more positive than at pre-test. Again, the effect size for the EM condition was larger than for the control condition (EM condition: $t=-3.48,95 \% \mathrm{Cl}[-.77--.20], p=.001$, Cohen's $d=-.30$; control: $t=-.68,95 \% \mathrm{Cl}[-.39-.19], p=.50$, Cohen's $d=-.05)$. 
Chapter 5

As predicted, both vividness and emotionality ratings decreased. However, the results did not reveal a difference between both conditions. This decrease in vividness and negativity could be attributed to the time interval or non-specific factors. To examine whether there is a relation between vividness and emotionality, Pearson's $r$ was calculated. A correlation was found between post vividness and emotionality ratings for the EM condition ( $r=-0.38, n=41$, $p<.05)$, but not for the control condition $(r=-0.08, n=41, p>.05)$.

Table 5.1 Mean Scores (and SDs) and Cls on Vividness and Emotionality, Before and After Performing Eye Movements or Control.

\begin{tabular}{lcccccccc}
\hline & \multicolumn{4}{c}{ Vividness } & \multicolumn{4}{c}{ Emotionality } \\
\hline & Pre & Cl & Post & Cl & Pre & Cl & Post & Cl \\
\cline { 2 - 9 } EM $(n=41)$ & $7.73(1.16)$ & $7.36-8.10$ & $6.88(1.40)$ & $6.44-7.32$ & $2.20(1.63)$ & $1.68-2.71$ & $2.68(1.69)$ & $2.15-3.22$ \\
Control $(n=41)$ & $7.83(1.34)$ & $7.40-8.26$ & $7.31(1.56)$ & $6.83-7.81$ & $2.76(1.95)$ & $2.14-3.37$ & $2.85(1.77)$ & $2.30-3.41$ \\
\hline Note. EM = Eye Movement condition & & & & & &
\end{tabular}

Misinformation. Means of correct answers and endorsed misinformation answers are depicted in Figure 5.1. An independent samples t-test revealed a statistically significant difference in the number of correct answers and the endorsement of misinformation. That is, confirming our hypothesis, control participants answered questions correctly more often than EM participants $(t(80)=4.00,95 \% \mathrm{Cl}[.53-1.75], p<.001$, Cohen's $d=-.88)$. EM participants significantly more often accepted misinformation than control participants $(t(80)=-3.47,95 \% \mathrm{Cl}[-1.34--.36], p$ $=.001$, Cohen's $d=.77$ ). A correlation was also found between the number of correct answers and number of endorsed misinformation items for the EM condition ( $r=-0.90, n=41, p<.001)$ and for the control condition $(r=-0.86, n=41, p<.001)$. We also calculated a Bayes factor (i.e., a comparison of how well two hypotheses predict the data) using a default prior of 0.71 . The larger this factor deviates from zero, the more support for the alternative hypothesis (Goodman, 1999). We found a $\mathrm{BF}_{10}=35.26$ indicating that our eye movement-misinformation effect is more in line with the alternative hypothesis than with the null hypothesis. Next to that, we examined the post-hoc power of our experiment. A post-hoc observed power analysis indicated a power of 0.93 for the Condition effect.

Exploratory analysis. We explored whether BDI scores served as a covariate. An independent samples t-test revealed no significant difference in BDI between the two conditions (EM: $M$ = 3.93, $S D=4.09$; control: $M=4.59, S D=3.71 ; t=.77,95 \% \mathrm{Cl}[-1.06-2.37], p=.45$, Cohen's $d$ $=-.17)$. An ANOOVA with BDI scores as covariate revealed no significant interaction effect on 


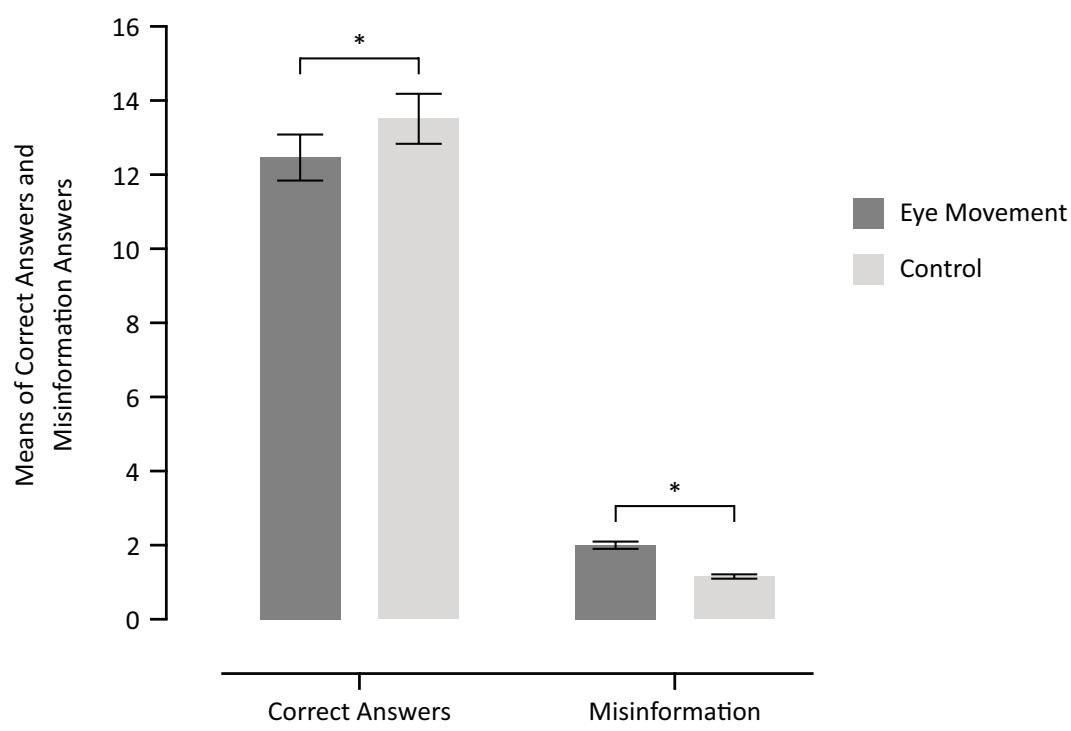

Figure 5.1 Means of correct answers and misinformation items for eye movement condition and control condition. Error bars show 95\% confidence intervals.

the number of correct answers $\left(F(1,78)=2.42, p=.12, \eta^{2}\right.$ partial $\left.=.03\right)$ and no significant main effect of BDI scores $\left(F(1,78)=.002, p=.97, \eta^{2}\right.$ partial $\left.=.000\right)$. A main effect of Condition emerged $\left(F(1,78)=14.60, p<.001, \eta^{2}\right.$ partial $\left.=.158\right)$. For misinformation acceptance, a similar pattern was found. An ANOOVA with BDI scores as covariate revealed no significant interaction effect on misinformation acceptance $\left(F(1,78)=.82, p=.37, \eta^{2}\right.$ partial $\left.=.01\right)$ and no significant main effect of BDI scores $\left(F(1,78)=.001, p=.98, \eta_{\text {partial }}^{2}=.000\right)$. A main effect of Condition emerged $\left(F(1,78)=8.79, p=.004, \eta^{2}\right.$ partial $\left.=.101\right)$. BDI scores were not statistically correlated with VAS ratings (pre, post, and mean change), number of correct answers or number of endorsed misinformation items (all ps > .05), which suggests that BDI scores did not affect memory scores.

\section{DISCUSSION}

Individuals who are treated with EMDR may be unintentionally exposed to misinformation incorporated in follow-up questions of a therapist. The aim of the current study was to examine susceptibility to misinformation after performing eye movements. We found an unwanted drawback of such eye movements. That is, participants who had engaged in eye 


\section{Chapter 5}

movements more often endorsed misinformation than control participants.

Our finding seems to contradict the results of Parker and colleagues (2009). As previously stated, participants in the eye movement conditions were more resistant to the misinformation effect than the controls. Our design differs from Parker et al. (2009), as we intended to use a sequence that mimics what might happen during an EMDR session. Specifically, in such sessions, after (and not before) performing eye movements, clients might be confronted with (suggestive) misinformation during the follow-up interview. Thus, the important question is whether eye movements may make people more susceptible to misinformation. We are the first showing that this is, indeed, the case. Eye movements might have an undesirable drawback.

Furthermore, the results could also be explained in terms of the discrepancy detection principle (Tousignant et al., 1986). According to this principle, the larger the discrepancy between an actual memory and misleading information, the less susceptible individuals will be to create false memories. It is likely that this discrepancy can decrease as the memory becomes less vivid, and hence it will be more difficult to detect the discrepancy. The consequence is that participants will become more susceptible to incorporate misinformation in their memory reports. It is reasonable to assume that eye movements increase reporting of misinformation because they interfere with the vividness of true memories (Lee \& Cuijpers, 2013).

In our study, participants also rated vividness and emotionality of the video before and after performing eye movements. The video was perceived as less vivid and more positive at post-test for both the eye movement and control condition. Often, such effects are more pronounced in participants performing eye movements (van den Hout \& Engelhard, 2012). In line with this, effect sizes for decreases in vividness and negative emotions in our study were substantially larger for the eye movement than for the control condition.

Our study was based on previous work on EMDR and hence, we used effect sizes found in that research. A limitation of this could be that although our study had adequate power and although the sample size was based on previous effect sizes, recent research has suggested that reported effect sizes in studies are likely to be an overestimation of actual effect sizes (Matzke et al., 2015). Hence, future studies might attempt to replicate this study using a larger sample size.

To conclude, research has suggested that EMDR, and thus eye movements, are effective as a treatment for PTSD, but the current work is the first showing that eye movements can have adverse effects. This finding combined with recent work showing that individuals 
with PTSD are at increased risk to create false memories (Otgaar et al., 2017), and work showing that therapists have poor knowledge on issues concerning memory (Patihis et al., 2014), stresses the importance to conduct follow-up research on the drawbacks of EMDR.

Even when therapists have correct knowledge about the functioning of memory and refrain from suggestive questioning during therapy, it might still be the case that an intervention itself may increase the production of false memories. The following chapter will therefore focus on the link between performing eye movements and spontaneous false memories by using a paradigm that does not rely on external pressure, but on automatic processes that can foster the formation of false memories. 


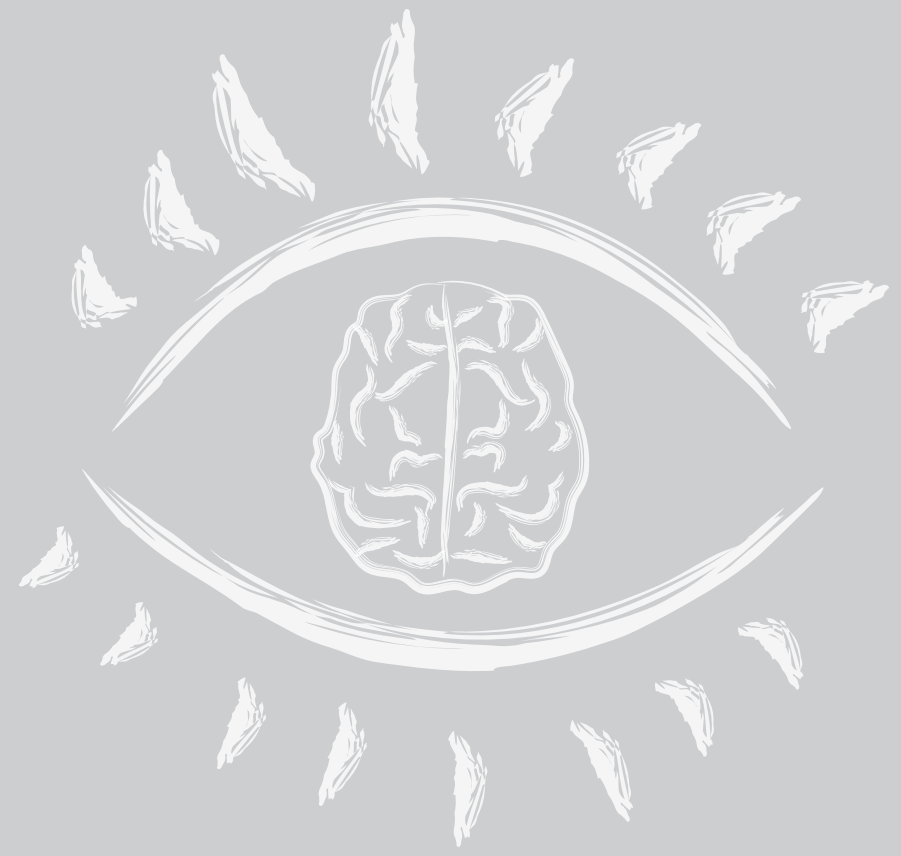




\section{CHAPTER 6}

\section{Eye movements and spontaneous false memories}

Published as:

Houben, S. T. L., Otgaar, H., Roelofs, J., Smeets, T., \& Merckelbach, H. (2020). Increases of correct memories and spontaneous false memories due to eye movements when memories are retrieved after a time delay. Behavior Research and Therapy, 125, 103546. https://doi.org/10.1016/j.brat.2019.103536 



\section{INTRODUCTION}

Eye Movement Desensitization and Reprocessing (EMDR; Shapiro, 1989) is a widely used treatment for trauma-related disorders (de Jongh \& ten Broeke, 2016). During EMDR, patients retrieve traumatic memories and are instructed to concentrate on the core cognitions they have about themselves as well as on the emotions, thoughts, and bodily sensations that emerge (Shapiro \& Maxfield, 2002). Simultaneously, the therapist induces eye movements by moving the index finger horizontally in front of the patient's visual field or by using an electronic device such as the EMDR kit (see https://www.emdrkit.com). Typically, eye movements lead to a reduction in self-reported vividness and emotionality of the traumatic memory (e.g., van den Hout \& Engelhard, 2012).

Laboratory studies generally support the beneficial effects (i.e., reductions in vividness and emotionality) of eye movements. In these studies, healthy participants are asked to retrieve a negative autobiographical memory and rate its vividness and emotionality on a visual analogue scale (VAS). Following this, participants perform horizontal eye movements (i.e., tracking a moving dot on a computer screen) for several episodes of $24 \mathrm{~s}$ or not (i.e., control condition; van den Hout \& Engelhard, 2012), and rate the vividness and emotionality of their memory on a VAS once more. In general, eye movements decrease participants' memory vividness and emotionality (Lee \& Cuijpers, 2013).

According to the working memory account, the therapeutic power of eye movements is due to their interference with working memory (Gunter \& Bodner, 2008; van den Hout \& Engelhard, 2012). This account stipulates that performing eye movements and retrieving an autobiographical memory both require working memory capacity. As working memory capacity is limited (Baddeley, 1998), less capacity is available for memory retrieval when it is accompanied by eye movements. This trade-off between memory and eye movements will manifest itself in reduced vividness and emotionality of the traumatic memory, a phenomenon called imagination deflation (van den Hout \& Engelhard, 2012).

\section{Eye Movements and False Memory Production}

Using a fear condition procedure, Leer and colleagues (2017; Experiment 2) showed that eye movements, as used in EMDR, decrease memory accuracy. Research on adverse therapy effects has great practical relevance (e.g., Moritz et al., 2015) and with this in mind, researchers became interested in whether EMDR, specifically eye movements, might increase individual's 


\section{Chapter 6}

susceptibility to false memories (i.e., memories of non-experienced details or events; Loftus, 2004). One distinct possibility is that eye movements, through its taxing of working memory, make participants more susceptible to incorporate misinformation into their memory report. Houben and colleagues (2018) found suggestive evidence for such an effect. In their experiment, participants saw a car crash video, then performed eye movements or not, and finally were exposed to misinformation about the video. Eye movements increased endorsement of misinformation. However, it remains to be seen whether the false memory creating potential of eye movements is a robust phenomenon. Importantly, two recent direct replications (Calvillo \& Emami, 2019; van Schie \& Leer, 2019) failed to reproduce the phenomenon. Clearly, these contradictory findings require follow-up research. In this previous work on false memory and eye movements, researchers attempted to elicit false memories by providing participants with misinformation. So far, few studies examined whether eye movements might amplify false memories that arise spontaneously (i.e., without any external pressure). Van Schie and Leer (2019) also noted this omission and recommended to use methods that rely on automatic memory dynamics, rather than external pressure.

One of the most popular and reliable methods to elicit spontaneous false memories is the Deese-Roediger-McDermott (DRM; Deese, 1959; Roediger \& McDermott, 1995) paradigm. In this paradigm, participants receive word lists containing associated words (e.g., bed, tired, dream, etc.) that are all linked to a non-presented word, termed the critical lure (i.e., sleep). Free recall and recognition tests have demonstrated that many participants falsely remember the critical lure (e.g., Gallo, 2010).

Knott and Dewhurst (2007a; Experiment 2) had participants study DRM word lists under full attention and carried out a recognition test in full or divided attention (i.e., generate number during recognition). Divided attention at retrieval led to lower correct recognition rates, and, more interestingly, increased false recognition rates. Shah and Knott (2018) replicated this and found that divided attention at retrieval (i.e., generating numbers during retrieval) led to higher rates of both negative and neutral false memories. The authors also measured metacognitive remember/know responses to examine whether false reports were accompanied by vivid, conscious recollections (i.e., remember response) and/or a strong sense of familiarity (i.e., know response). It appeared that divided attention increased recollection but not familiarity aspect of both negative and neutral false memories (Shah \& Knott, 2018).

So far, only few authors employed the DRM paradigm to systematically examine the link between eye movements and spontaneous false recognition. In the studies of Parker and 
Dagnall (2007; 2012), participants heard the associated words and were randomly assigned to either a horizontal, vertical, or no eye movement condition. Participants' memory was tested with a recognition task. Horizontal eye movements resulted in higher hit rates and reduced false recognition rates (i.e., false memories) compared with vertical eye movements and no eye movements condition (see also Christman et al., 2004; Nieuwenhuis et al., 2013).

However, previous work on eye movements and false memories elicited by a DRM paradigm suffers from two important limitations. First, during real life EMDR sessions, patients retrieve their memory while simultaneously performing eye movements. However, in the laboratory studies of Parker and Dagnall (2007; 2012), eye movements were carried out after they received the associated words. A second limitation is that their studies involved neutral (false) memory material, whereas EMDR is typically applied to traumatic memories.

\section{The Current Study}

The aim of the current study was to examine whether eye movements, as used in EMDR, may amplify spontaneous false memory susceptibility. In contrast to previous studies (e.g., Parker \& Dagnall, 2007), participants had to perform eye movements during the retrieval of memories. Furthermore, participants were presented with neutral and emotionally negative DRM lists.

The reason for using emotionally negative and neutral word lists was twofold. First, as said before, EMDR is usually applied to emotionally negative memories. Second, research on the effects of valence on false memory formation has consistently shown that negative valence affects false memory rates (for an overview see Bookbinder \& Brainerd, 2016). Specifically, during free recall, false memory rates are higher for neutral than for negative stimuli, whereas the reverse occurs during recognition. We anticipated to find a similar pattern of findings.

Based on previous findings on divided attention and false memory generation (Shah \& Knott, 2018), we expected that participants in the eye movement condition would be more susceptible to false memory creations as compared to control participants (on free recall and recognition test). Our reasoning was that eye movements would engender divided attention, leading participants to focus on the gist rather than the details of the retrieved memories. Such enhancement of gist memory would make participants more prone to false memory production (Brainerd \& Reyna, 2002). In the current study, we also measured remember/ know responses. We predicted that eye movements would lead to fewer remember and know responses for correct memories than when no eye movements were performed (Knott 


\section{Chapter 6}

\& Dewhurst, 2007b). For critical lures, we expected to find that eye movements would lead to more remember responses than know responses.

In clinical practice, there is often a considerable time interval between encoding of the traumatic event and receiving treatment, such as EMDR. To approach this situation more closely (i.e., encoding an event and retrieving the corresponding memory days later), free recall and recognition test were administered in Experiment 2 after a delay of 48 hrs. We expected higher false memory levels in Experiment 2 than in Experiment 1. Our expectation is derived from Fuzzy Trace Theory (FTT; Brainerd \& Reyna, 2002), which stipulates that it will become more difficult to retrieve verbatim traces when time passes and individuals have to rely on gist traces. The increased reliance on gist traces will likely stimulate false memory production.

\section{Experiment 1}

\section{METHOD}

\section{Participants}

The experiments were preregistered at the Open Science Framework (see https://osf.io/gx7te/ registrations). The preregistration included a 2 (Condition: Eye Movement vs. Control) $\times 2$ (Valence: Neutral vs. Negative) × 2 (Time: Immediate vs. Delayed) mixed design and power analysis. Based on previous research (Parker et al., 2012; study on eye movements and false memories with an effect size of $\eta^{2}$ partial $=0.04$ ), an a priori power analysis using $G^{*}$ Power (Faul et al., 2007), with a medium effect size ( $f=0.25$; Howe et al., 2009) and a power of 0.80, showed that a total sample size of 136 participants was needed. As participants were not randomly assigned for the variable Time (see below), two separate experiments are reported (Experiment $1 n=72$, Experiment $2 n=68$ ).

The sample of Experiment 1 consisted of 16 psychology and 56 medicine undergraduate students of Maastricht University $\left(N=72, M_{\text {age }}=21.07, S D=2.27\right.$, range 18 - 30, 66 women; $n=36$ per condition). Participants were asked whether they had prior knowledge of what EMDR entails. Five participants of the experimental condition indicated to have knowledge of EMDR, but these participants were not excluded as previous research showed that prior knowledge does not affect any memory effects of EMDR (Littel, van Schie, \& van den Hout, 2017) ${ }^{5}$. All participants provided written consent and received course credits or a 
financial reimbursement of $€ 7,50$ for their participation. All participants were native Dutch speakers. The experiment was approved by the standing ethical committee of the Faculty of Psychology and Neuroscience.

\section{Materials}

All data and materials are available at the Open Science Framework at https://osf.io/gx7te.

Deese/Roediger-McDermott (DRM) Paradigm. Five neutral DRM lists (critical lures: bread, window, sweet, smoke, foot) and five negative DRM lists (critical lures: murder, pain, punishment, death, cry) were used as stimulus material. These Dutch DRM word lists (https://osf.io/n2fve) are known to reliably induce false memories (see e.g., Otgaar et al., 2012). Each DRM list included ten associated words. The order of word lists presentation was counterbalanced (i.e., neutral DRM lists - negative DRM lists; negative DRM lists neutral DRM lists). Mean word frequency of neutral and negative critical lures did not differ statistically $(t(8)=0.22, n s)$.

Eye Movement Task. To simulate the eye movement component of EMDR in a standardized way, an EMDR kit version 2.0 was used (see https://www.emdrkit.com). This kit is also employed for therapeutic purposes. A white dot was presented on a bar (length light bar $70 \mathrm{~cm}$ ) and moved from left to right with one movement per second (speed: 12/15; one movement per second), during four episodes of $24 \mathrm{~s}$ with a $10 \mathrm{~s}$ interval. The duration was in line with previous research (van den Hout \& Engelhard, 2012) and largely converges to general procedures in EMDR therapy. All participants sat at a $30 \mathrm{~cm}$ distance from the EMDR kit. The researcher checked whether participants complied with the eye movement instructions by monitoring the participants' eye movements. Control participants viewed a blank screen on a computer.

Free Recall. During the four episodes of performing eye movements (or not), participants were asked to freely recall the words they could remember at this point in time (see for details below). The free recall was audiotaped. Free recall was scored in a liberal manner. That is, if the presented word was "shoes" and the participant recalled the word "shoe" this was scored as a correct response (i.e., from plural to singular or vice versa). If we would have scored in a conservative manner (i.e., exact reproduction), the word "shoe" would have been treated as an incorrect response. Repetition of the same word was not included in total words recalled.

Recognition Test. The recognition test (https://osf.io/5desv) for the DRM lists contained 


\section{Chapter 6}

of 78 words including 40 correct items (e.g., flour, blood), ten critical lures (e.g., bread, murder), ten non-presented related items (e.g., syrup, shooting), and 18 non-presented unrelated items (e.g., bus, friend). The recognition test was administered orally. Additionally, whenever participants indicated to recognize a word, they were asked to make a remember/ know response. It was explained to participants that the remember option referred to vivid, clear recollections and the know option to memories accompanied by a strong sense of familiarity (Tulving, 1985).

Dissociative Experiences Scale. The Dissociative Experiences Scale (DES; Bernstein \& Putnam, 1986; https://osf.io/h3xs6) was included for explorative analyses. It measures selfreported dissociative tendencies in, for example, memory (e.g., not remembering whether you have actually done something, or only thought about it). The DES contains 28 items that ask participants to rate the frequency of these experiences. In the current experiment, participants used a $100-\mathrm{mm}$ scale to indicate the percentage of time they experienced such dissociative phenomena ( 0 = not at all; 100 = very much). Higher DES scores indicate a higher frequency of self-reported dissociative experiences. The internal consistency of the DES in the current study was good (Cronbach's $\alpha=.90$ ). Results of the DES can be found on the OSF (https://osf.io/5x6qt).

\section{Design and Procedure}

The experiment relied on a 2 (Condition: Eye movements vs. Control) $\times 2$ (Valence: Negative vs. Neutral) split-plot design. Condition was a between-subjects factor and Valence a withinsubjects factor. The dependent variables were (a) correct recall and hit rates (i.e., correct responses during free recall and recognition, respectively), (b) false recall and false recognition (i.e., acceptance of critical lures during free recall and recognition, respectively), and (c) extra intrusions on free recall and recognition (i.e., reporting/acceptance of unpresented words). Additionally, we examined endorsement of remember and know responses.

Participants were randomly assigned to either the eye movement ( $n=36$ ) or the control condition $(n=36)$ and were tested individually in a quiet laboratory room. They were informed that they were about to take part in a word recognition study. After obtaining written informed consent, participants completed the DES. Then, they were instructed to pay close attention to the words presented on the computer screen. Participants were presented with five negative DRM lists and five neutral DRM lists, presented in a blocked order. Half of the participants first viewed the negative word lists, followed by the neutral word lists. The 
other half of the participants received the reversed order. The words appeared in the middle of the screen for 2 s (Knott \& Dewhurst, 2007b; Nieuwenhuis et al., 2013) followed by a blank screen for 2 s. After each list, a "+" sign was shown. After viewing the DRM word lists, participants performed a filler task (playing the game Bejeweled) for five minutes. This served as a distraction task and to ensure a delay between the stimuli and follow-up tasks. After five minutes, the participants were told to stop and the free recall test began.

All participants received the instruction to recall words they could remember at this point in time, for four episodes of $24 s$ with a $10 \mathrm{~s}$ break between episodes. The experimenter stressed that it did not matter whether they recalled the words in a different order or repeated the same words. Participants were instructed to focus on their emotions, feelings, bodily sensations, and thoughts when recalling the words, similar to the EMDR protocol (de Jongh \& ten Broeke, 2016). Participants in the eye movement condition were additionally instructed to follow the dot with their eyes as it moved from left to right on the EMDR kit and were asked to keep their head still. The distance to the EMDR kit was $30 \mathrm{~cm}$. Compliance with these instructions was monitored by the experimenter. Participants in the control condition viewed a blank screen. Following the free recall phase, all participants completed a filler task for five minutes (playing Bejeweled). After this, they had to complete the recognition test. The experimenter recited words and participants were instructed to answer "yes" if they recalled the word, or "no" if they did not recall the word. If a "yes" response was made, participants were asked to provide a remember or know response. Participants were debriefed after all participants were tested.

\section{RESULTS}

Mean proportions of recall and recognition rates are shown in Table 6.1. The various memory parameters were subjected to 2 (Condition: Eye Movements vs. Control) $\times 2$ (Valence: Negative vs. Neutral) repeated measures ANOVAs. Most effects, including the critical main effects of condition and interaction effects of condition with valence, did not reach statistically significant levels and had low Bayes Factors (BF; i.e., < 1.00; BF expresses the likelihood of the data under $\mathrm{H}_{1}$ relative to $\mathrm{H}_{0}$; JASP team 2019). Therefore, we do not elaborate on the main and interaction effects of all ANOVAs here. Instead, we refer to Table $\mathbf{6 . 2}$ for a summary of the data. 


\section{Chapter 6}

Table 6.1 Mean proportions and standard deviations per condition for recall and recognition of Experiment 1.

\begin{tabular}{llcc}
\hline & & EM condition $(\boldsymbol{n}=\mathbf{3 6})$ & Control condition $(\boldsymbol{n}=\mathbf{3 6})$ \\
\hline Recall & & & \\
True recall & Negative & $0.26(.12)$ & $0.31(.14)$ \\
False recall & Neutral & $0.29(.14)$ & $0.33(.15)$ \\
& Negative & $0.21(.18)$ & $0.26(.24)$ \\
& Neutral & $0.29(.21)$ & $0.33(.23)$ \\
Recognition & & \\
Hit rate & Negative & $0.78(.14)$ & $0.86(.11)$ \\
& Neutral & $0.79(.13)$ & $0.80(.14)$ \\
Critical lures & Negative & $0.74(.24)$ & $0.72(.28)$ \\
& Neutral & $0.73(.23)$ & $0.65(.25)$ \\
\hline
\end{tabular}

Note. EM = Eye movement.

There were a few exceptions to this overall pattern. For false recall (i.e., critical lures), a 2 (Condition: Eye Movements vs. Control) × 2 (Valence: Negative vs. Neutral) repeated measures ANOVA revealed a statistically significant main effect of valence. Overall, participants falsely recalled more neutral lures $(M=0.31, S D=0.14)$ than negative lures $(M=0.28, S D=0.13$; $\left.F(1,70)=5.11, p=.027, \eta_{\text {partial }}^{2}=.068, \mathrm{BF}_{10}=2.24\right) . \mathrm{A} 2$ (Condition: Eye Movements vs. Control) $\times 2$ (Valence: Negative vs. Neutral) repeated measures ANOVA performed on the know responses related to hits yielded a statistically significant main effect of valence. Participants provided more know responses for negative hits $(M=0.16, S D=0.12)$ than for neutral hits $\left(M=0.10, S D=0.10 ; F(1,70)=21.91, p<.001, \eta^{2}\right.$ partial $\left.=.238, \mathrm{BF}_{10}=1752.86\right) . \mathrm{A} 2$ (Condition: Eye Movements vs. Control) $\times 2$ (Valence: Negative vs. Neutral) repeated measures ANOVA performed on the know responses related to false recognition yielded a statistically significant main effect of valence. That is, participants provided more know responses for negative critical lures $(M=0.27, S D=0.23)$ than for neutral critical lures $(M=0.19, S D=0.19 ; F(1,70)=5.66$, $\left.p=.020, \eta_{\text {partial }}^{2}=.075, \mathrm{BF}_{10}=2.60\right)$.

\section{DISCUSSION}

In Experiment 1, eye movements did not increase the susceptibility to spontaneous false memory formation. In line with previous studies, participants falsely recalled more neutral lures than negative lures (Pesta, Murphy, \& Sanders, 2001). We also found that know responses more often accompanied hits and false recognition of negative material than hits and false recognition of neutral material. 
Table 6.2 Results Statistical Analyses of Experiment 1.

\begin{tabular}{|c|c|c|c|c|c|}
\hline & & $\boldsymbol{F}$ & $p$ & Partial eta ${ }^{2}$ & $B F_{10}$ \\
\hline \multicolumn{6}{|l|}{ Free Recall } \\
\hline \multirow[t]{3}{*}{ True recall } & Main effect condition & 3.42 & 0.069 & 0.047 & 0.74 \\
\hline & Main effect valence & 1.62 & 0.207 & 0.023 & 0.41 \\
\hline & Interaction effect condition $\times$ valence & 0.08 & 0.781 & 0.001 & 0.08 \\
\hline \multirow[t]{3}{*}{ False recall } & Main effect condition & 1.20 & 0.278 & 0.017 & 0.34 \\
\hline & Main effect valence & 5.11 & 0.027 & 0.068 & 2.24 \\
\hline & Interaction effect condition $\times$ valence & 0.06 & 0.802 & 0.001 & 0.09 \\
\hline \multicolumn{6}{|l|}{ Recognition } \\
\hline \multirow[t]{3}{*}{ Hit rates } & Main effect condition & 2.98 & 0.089 & 0.041 & 0.81 \\
\hline & Main effect valence & 1.83 & 0.180 & 0.025 & 0.42 \\
\hline & Interaction effect condition $\times$ valence & 3.26 & 0.075 & 0.044 & 0.34 \\
\hline \multirow[t]{3}{*}{ False recognition } & Main effect condition & 0.96 & 0.331 & 0.014 & 0.41 \\
\hline & Main effect valence & 1.68 & 0.199 & 0.023 & 0.38 \\
\hline & Interaction effect condition $\times$ valence & 1.24 & 0.270 & 0.017 & 0.07 \\
\hline \multirow[t]{3}{*}{$\mathrm{R}$ response for hits } & Main effect condition & 2.42 & 0.125 & 0.033 & 0.65 \\
\hline & Main effect valence & 2.47 & 0.121 & 0.034 & 0.59 \\
\hline & Interaction effect condition $\times$ valence & 0.87 & 0.354 & 0.012 & 0.12 \\
\hline \multirow[t]{3}{*}{$\mathrm{R}$ response for $\mathrm{CL}$} & Main effect condition & 0.06 & 0.810 & 0.000 & 0.27 \\
\hline & Main effect valence & 0.15 & 0.700 & 0.002 & 0.19 \\
\hline & Interaction effect condition $\times$ valence & 0.29 & 0.589 & 0.004 & 0.01 \\
\hline \multirow[t]{3}{*}{ K response for hits } & Main effect condition & 0.18 & 0.671 & 0.003 & 0.26 \\
\hline & Main effect valence & 21.91 & $<.001$ & 0.238 & 1752.86 \\
\hline & Interaction effect condition $\times$ valence & 0.20 & 0.659 & 0.003 & 0.08 \\
\hline \multirow[t]{3}{*}{$\mathrm{K}$ response for $\mathrm{CL}$} & Main effect condition & 1.65 & 0.203 & 0.23 & 0.44 \\
\hline & Main effect valence & 5.66 & 0.020 & 0.75 & 2.60 \\
\hline & Interaction effect condition $\times$ valence & 0.30 & 0.585 & 0.004 & 0.13 \\
\hline Intrusions & & $t$ & $p$ & $95 \% \mathrm{Cl}$ & \\
\hline Free recall & & 1.79 & 0.077 & {$[-.07,1.41]$} & 0.96 \\
\hline \multirow[t]{2}{*}{ Recognition } & Related & 0.82 & 0.418 & {$[-.36, .86]$} & 0.32 \\
\hline & Unrelated & -0.68 & 0.496 & {$[-.07, .03]$} & 0.28 \\
\hline
\end{tabular}

Notes. $\mathrm{R}=$ remember response; $\mathrm{K}=$ know response; $\mathrm{CL}=$ critical lure; $\mathrm{Cl}=$ confidence interval; $\mathrm{BF}_{10}=$ Bayes Factor that expresses the likelihood of the data under $\mathrm{H}_{1}$ relative to $\mathrm{H}_{0}$.

To approach the therapeutic situation more closely (i.e., encoding an event and retrieving the corresponding memory days later), we conducted Experiment 2 that deviated from Experiment 1 in that here we used a longer time window (i.e., 48 hours) between memory encoding and eye movement manipulations. 


\section{Experiment 2}

\section{METHOD}

\section{Participants}

The preregistration included a 2 (Condition: Eye Movement vs. Control) $\times 2$ (Valence: Neutral vs. Negative) $\times 2$ (Time: Immediate vs. Delayed) mixed design and power analysis. Based on previous research (Parker et al., 2012; study on eye movements and false memories with an effect size of $\eta^{2}$ partial $=0.04$ ), an a priori power analysis using $G *$ Power (Faul et al., 2007), with a medium effect size ( $f=0.25$; Howe et al., 2009) and a power of 0.80 , showed that a total sample size of 136 participants was needed. As said before, participants were not randomly assigned for the variable Time, and so two separate experiments are reported (Experiment $1 n=72$, Experiment $2 n=68$ ). In the Experiment 2, the sample included 21 psychology and 47 medicine undergraduate students of Maastricht University $\left(N=68, M_{\text {age }}=20.51, S D=2.00\right.$, range 18 - 26, 59 women; $n=34$ per condition). Five participants indicated to have knowledge about EMDR, but they were not excluded 6 .

All participants provided written consent and received course credits or a financial reimbursement of $€ 7,50$ for their participation. All participants were native Dutch speakers. Participants in Experiment 2 did not participate in Experiment 1. The experiment was approved by the standing ethical committee for the Faculty of Psychology and Neuroscience.

\section{Materials}

Materials were the same as in Experiment 1 and the data and materials can be found at the Open Science Framework https://osf.io/gx7te.

\section{Design and Procedure}

The design and procedure of Experiment 2 were similar to Experiment 1, except for the following changes. The procedure involved two sessions with a 48 hours interval in between. During session one, participants completed the DES and viewed the DRM word lists. During session 2 (48 hrs. later), participants were randomly assigned to either the eye movement $(n=34)$ or control condition $(n=34)$ and completed the free recall and recognition test and received the same instructions as in Experiment 1. Participants were debriefed after all participants were tested. 


\section{RESULTS}

Mean proportions of recall and recognition rates are shown in Table 6.3. The results are summarized in Table 6.4 .

\section{Free Recall}

True Recall. A 2 (Condition: Eye Movements vs. Control) × 2 (Valence: Negative vs. Neutral) repeated measures ANOVA was performed on correct recall. A statistically significant interaction effect emerged, $F(1,66)=6.80, p=.011, \eta^{2}$ partial $=.093, \mathrm{BF}_{10}=5.06$. Simple effect analysis revealed that participants in the eye movement condition recalled more neutral items $(M=0.23, S D=0.17)$ than control participants $(M=0.12, S D=0.13), F(1,66)=9.80, p$ $\left.=.003, \eta_{\text {partial }}^{2}=.129\right)$. No statistical difference was found for negative items $(F(1,66)=0.14$, $p=.71, \eta_{\text {partial }}^{2}=.002$ ).

False Recall. A 2 (Condition: Eye Movements vs. Control) $\times 2$ (Valence: Negative vs. Neutral) repeated measures ANOVA yielded a non-significant interaction, $F(1,66)=0.58, p=$ $.45, \eta^{2}$ partial $=.009$. A statistically significant main effect of condition emerged, $F(1,66)=4.43$, $p=.039, \eta_{\text {partial }}^{2}=.063, \mathrm{BF}_{10}=1.19$. That is, participants in the eye movement condition $(M=$ $0.27, S D=0.17)$ falsely recalled more critical lures than control participants $(M=0.19, S D=$ $0.14)$. No main effect of valence was found, $F(1,66)=0.06, p=.80, \eta^{2}{ }_{\text {partial }}=.001$.

Intrusions. An independent samples t-test on the number of intrusions yielded no statistical difference between the groups $(t(66)=0.48, p=.63,95 \% \mathrm{Cl}[-1.12,1.83])$.

\section{Recognition}

Hit Rates. A 2 (Condition: Eye Movements vs. Control) $\times 2$ (Valence: Negative vs. Neutral) repeated measures ANOVA yielded neither a statistically significant interaction effect, $F(1,66)$ $=0.08, p=.78, \eta^{2}$ partial $=.001$, nor a main effect for condition, $F(1,66)=2.33, p=.13, \eta^{2}$ partial $=$ .034. A main effect of valence emerged, $F(1,66)=9.61, p=.003, \eta^{2}$ partial $=.127, \mathrm{BF}_{10}=14.48$, such that more negative words $(M=0.70, S D=0.16)$ than neutral words $(M=0.62, S D=0.20)$ were recognized.

False Recognition. A 2 (Condition: Eye Movements vs. Control) × 2 (Valence: Negative vs. Neutral) repeated measures ANOVA found no statistically significant interaction effect, $F(1,66)=1.20, p=.28, \eta^{2}$ partial $=.018$. The main effect of condition was statistically significant, $F(1,66)=6.88, p=.011, \eta^{2}$ partial $=.094, \mathrm{BF}_{10}=3.84$. More specifically, participants in the eye 


\section{Chapter 6}

movement condition falsely recognized more critical lures $(M=0.77, S D=0.21)$ than control participants $(M=0.64, S D=0.22)$. A main effect of valence was also found, $F(1,66)=11.84$, $p=.001, \eta_{\text {partial }}^{2}=.152, \mathrm{BF}_{10}=31.42$, in that more negative $(M=0.77, S D=0.25)$ than neutral critical lures were recognized $(M=0.64, S D=0.29)$.

Intrusions. An independent samples t-test was performed on false alarms for related and unrelated non-presented items. Participants in the eye movement condition $(M=0.24$, $S D=0.12)$ reported statistically significant more related words than control participants $(M=$ $0.18, S D=0.12 ; t(66)=-2.10, p=.040,95 \% \mathrm{Cl}[-1.21,-0.30]$, Cohen's $\left.d=0.50, \mathrm{BF}_{10}=1.58\right)$. Also, participants in the eye movement condition $(M=0.23, S D=0.13)$ recognized more unrelated words than control participants $(M=0.13, S D=0.09 ; t(66)=-3.68, p<.001,95 \% \mathrm{Cl}[-2.81$, $-.83]$, Cohen's $\left.d=0.89, \mathrm{BF}_{10}=58.14\right)$.

Table 6.3 Mean proportions and standard deviations per condition for recall and recognition of Experiment 2.

\begin{tabular}{llll}
\hline & & EM condition $(\boldsymbol{n}=\mathbf{3 4})$ & Control condition $(\boldsymbol{n}=\mathbf{3 4})$ \\
\hline Recall & & & \\
True recall & Negative & $0.15(.12)$ & $0.14(.10)$ \\
False recall & Neutral & $0.23(.17)$ & $0.12(.13)$ \\
& Negative & $0.25(.22)$ & $0.20(.17)$ \\
& Neutral & $0.29(.21)$ & $0.18(.24)$ \\
Recognition & & \\
Hit rate & Negative & & \\
& Neutral & $0.73(.11)$ & $0.68(.20)$ \\
Critical lures & Negative & $0.65(.21)$ & $0.59(.19)$ \\
& Neutral & $0.82(.20)$ & $0.72(.28)$ \\
\end{tabular}

Note. $\mathrm{EM}=$ Eye movement.

\section{Remember/Know}

Hit Rates. A 2 (Condition: Eye Movements vs. Control) × 2 (Valence: Negative vs. Neutral) repeated measures ANOVA on endorsement of remember options yielded a statistically significant interaction effect, $F(1,66)=6.20, p=.015, \eta_{\text {partial }}^{2}=.086, \mathrm{BF}_{10}=3.33$. Simple effect analyses revealed a simple main effect of condition, but only for neutral hits. That is, participants in the eye movement condition $(M=0.49, S D=0.25)$ provided more remember responses than control participants $(M=0.37, S D=0.18)$ for neutral hits, $F(1,66)=4.84, p=.031, \eta^{2}$ partial $=.068$, but not for negative hits $\left(F(1,66)=1.07, p=.30, \eta^{2}{ }_{\text {partial }}=.016\right)$.

As for endorsement of know options, a 2 (Condition: Eye Movements vs. Control) $x$ 2 (Valence: Negative vs. Neutral) repeated measures ANOVA yielded a statistically significant 
Table 6.4 Results Statistical Analyses of Experiment 2.

\begin{tabular}{|c|c|c|c|c|c|}
\hline & & $\boldsymbol{F}$ & $p$ & Partial eta ${ }^{2}$ & $B F_{10}$ \\
\hline \multicolumn{6}{|l|}{ Free Recall } \\
\hline \multirow[t]{3}{*}{ True recall } & Main effect condition & 9.80 & 0.003 & 0.129 & 2.81 \\
\hline & Main effect valence & 0.14 & 0.71 & 0.002 & 0.33 \\
\hline & Interaction effect condition $\times$ valence & 6.80 & 0.011 & 0.093 & 5.06 \\
\hline \multirow[t]{3}{*}{ False recall } & Main effect condition & 4.43 & 0.039 & 0.063 & 1.19 \\
\hline & Main effect valence & 0.06 & 0.80 & 0.001 & 0.19 \\
\hline & Interaction effect condition $\times$ valence & 0.58 & 0.45 & 0.009 & 0.06 \\
\hline \multicolumn{6}{|l|}{ Recognition } \\
\hline \multirow[t]{3}{*}{ Hit rates } & Main effect condition & 2.33 & 0.13 & 0.034 & 0.62 \\
\hline & Main effect valence & 9.61 & 0.003 & 0.127 & 14.48 \\
\hline & Interaction effect condition $\times$ valence & 0.08 & 0.78 & 0.001 & 0.04 \\
\hline \multirow[t]{3}{*}{ False recognition } & Main effect condition & 6.88 & 0.011 & 0.094 & 3.84 \\
\hline & Main effect valence & 11.84 & 0.001 & 0.152 & 31.42 \\
\hline & Interaction effect condition $\times$ valence & 1.20 & 0.28 & 0.018 & 0.43 \\
\hline \multirow[t]{3}{*}{$\mathrm{R}$ response for hits } & Main effect condition & 4.84 & 0.031 & 0.068 & 0.33 \\
\hline & Main effect valence & 1.07 & 0.30 & 0.016 & 0.29 \\
\hline & Interaction effect condition $\times$ valence & 6.20 & 0.015 & 0.086 & 0.47 \\
\hline \multirow[t]{3}{*}{$\mathrm{R}$ response for $\mathrm{CL}$} & Main effect condition & 27.03 & $<.001$ & 0.291 & 6864.16 \\
\hline & Main effect valence & 1.00 & 0.32 & 0.015 & 0.29 \\
\hline & Interaction effect condition $\times$ valence & 0.00 & 1.00 & 0.000 & $4.06^{-5}$ \\
\hline \multirow[t]{3}{*}{$\mathrm{K}$ response for hits } & Main effect condition & 0.514 & 0.464 & 0.008 & 0.31 \\
\hline & Main effect valence & 6.246 & 0.015 & 0.086 & 2.16 \\
\hline & Interaction effect condition $\times$ valence & 11.32 & 0.001 & 0.146 & 17.02 \\
\hline \multirow[t]{3}{*}{$\mathrm{K}$ response for $\mathrm{CL}$} & Main effect condition & 11.23 & 0.001 & 0.145 & 11.84 \\
\hline & Main effect valence & 6.18 & 0.015 & 0.086 & 3.84 \\
\hline & Interaction effect condition $\times$ valence & 0.90 & 0.35 & 0.013 & 0.393 \\
\hline Intrusions & & $t$ & $p$ & $95 \% \mathrm{Cl}$ & \\
\hline Free recall & & 0.48 & 0.63 & {$[-1.12,1.83]$} & 0.28 \\
\hline \multirow[t]{2}{*}{ Recognition } & Related & -2.10 & 0.040 & {$[-1.21,-0.30]$} & 1.58 \\
\hline & Unrelated & -3.68 & $<.001$ & {$[-2.81,-.83]$} & 58.14 \\
\hline
\end{tabular}

Notes. $\mathrm{R}=$ remember response; $\mathrm{K}=$ know response; $\mathrm{CL}=$ critical lure; $\mathrm{Cl}=$ confidence interval. $\mathrm{BF}{ }_{10}=$ Bayes Factor that expresses the likelihood of the data under $\mathrm{H}_{1}$ relative to $\mathrm{H}_{0}$.

interaction effect, $F(1,66)=11.32, p=.001, \eta^{2}$ partial $=.146, \mathrm{BF}_{10}=17.02$. Simple effect analyses revealed a simple main effect of condition, but only for negative hits. That is, participants in the eye movement condition provided a know response to negative hits $(M=0.27, S D=0.12)$ more often than control participants $\left(M=0.20, S D=0.12 ; F(1.66)=5.94, p=.018, \eta_{\text {partial }}^{2}=\right.$ $.083)$. 


\section{Chapter 6}

False Recognition. A 2 (Condition: Eye Movements vs. Control) $\times 2$ (Valence: Negative vs. Neutral) repeated measures ANOVA was performed for remember responses related to false recognition. No significant interaction effect was found, $F(1,66)=0.00, p=1.00, \eta^{2}$ partial $=$ .000. However, a statistically significant main effect of condition was observed, $F(1,66)=27.03$, $p<.001, \eta_{\text {partial }}^{2}=.291, \mathrm{BF}_{10}=6864.16$. Participants in the eye movement condition provided more remember responses $(M=0.54, S D=0.24)$ than control participants $(M=0.27, S D=$ 0.17). No main effect of valence emerged, $F(1,66)=1.00, p=.32, \eta^{2}$ partial $=.015$.

A 2 (Condition: Eye Movements vs. Control) × 2 (Valence: Negative vs. Neutral) repeated measures ANOVA performed on the know responses related to false recognition yielded no significant interaction effect between valence and condition, $F(1,66)=0.90, p=$ $.35, \eta^{2}$ partial $=.013$. A statistically significant main effect of condition was found, $F(1,66)=11.23$, $p=.001, \eta_{\text {partial }}^{2}=.145, \mathrm{BF}_{10}=11.84$. More specifically, control participants provided more know responses to critical lures $(M=0.37, S D=0.18)$ than participants in the eye movement condition $(M=0.24, S D=0.16)$. A statistically significant main effect of valence was also found, $F(1,66)=6.18, p=.015, \eta^{2}$ partial $=.086, \mathrm{BF}_{10}=3.84$. More know responses for negative critical lures $(M=0.77, S D=0.25)$ than for neutral critical lures were observed $(M=0.64, S D=0.29)$.

An independent samples t-test was performed on the remember responses for related and unrelated words. For related words, participants in the eye movement condition $(M=0.09, S D=0.07)$ more often provided a remember response to related words than control participants $\left(M=0.03, S D=0.06 ; t(66)=-3.67, p<.001, C l[-.91,-.27]\right.$, Cohen's $d=0.92, B_{10}$ =56.06). The same pattern was found for unrelated words. Participants in the eye movement condition $(M=0.06, S D=0.06)$ provided a remember response to unrelated words more often than control participants $(M=0.03, S D=0.04 ; t(66)=-2.30, p=.024, C l[-.99, .07]$, Cohen's $\left.d=0.59, \mathrm{BF}_{10}=2.27\right)$.

An independent samples t-test performed on know responses for related words found no statistical difference between conditions $(t(66)=-0.23, p=.820)$. For unrelated words, a statistically significant difference emerged. Participants in the eye movement condition $(M=$ $0.17, S D=0.10)$ provided a remember response to unrelated words more often than control participants $\left(M=0.10, S D=0.09 ; t(66)=-3.00, p=.004, C l[-2.11,-.42]\right.$, Cohen's $d=0.73, B_{10}$ $=10.02)$.

\section{Exploratory Analyses}

Because participants in the eye movement condition frequently generated both more correct 
and false memories (i.e., critical lures) than did controls, we wondered whether their heightened false memory levels were an artefact of a positive response bias (Snodgrass \& Corwin, 1988). These analyses can be found on https://osf.io/89fyu.

\section{Comparison between Experiments}

In our preregistration, we were interested in the effect of time on spontaneous false memory formation. As said earlier, the preregistration included a 2 (Condition: Eye Movement vs. Control) $\times 2$ (Valence: Neutral vs. Negative) $\times 2$ (Time: Immediate Exp. 1 vs. Delayed Exp. 2) mixed design. Based on previous research (Parker et al., 2012; study on eye movements and false memories with an effect size of $\eta^{2}$ partial $\left.=0.04\right)$, an a priori power analysis using $G *$ Power (Faul et al., 2007), with a medium effect size ( $f=0.25$; Howe et al., 2009) and a power of 0.80 , showed that a total sample size of 136 participants was needed. Hence, we compared the data from Experiments $1(n=72)$ and $2(n=68)$ to examine the effect of time (total $N=$ 140). As no a priori hypotheses were formulated with regard to Remember/Know responses, results pertaining to these judgments can be found on the OSF (https://osf.io/2eayz).

\section{Free Recall}

True Recall. A 2 (Condition: Eye Movements vs. Control) × 2 (Valence: Negative vs. Neutral) $\times 2$ (Time: Immediate vs. Delayed) repeated measures ANOVA was performed on correct recall. The three-way interaction was non-significant, $F(1,136)=2.30, p=.13, \eta^{2}{ }_{\text {partial }}=.017 . \mathrm{A}$ statistically significant interaction effect between condition and time emerged, $F(1,136)=9.60$, $p=.002, \eta_{\text {partial }}^{2}=.066, \mathrm{BF}_{10}=3.21$. First, simple effect analysis revealed a statistically significant simple main effect of time for both conditions. That is, participants in the control condition in the immediate condition (Experiment $1 ; M=0.32, S D=0.10$ ) recalled more hits than control participants in Experiment $2(M=0.13, S D=0.09), F(1,136)=68.36, p<.001, \eta^{2}$ partial $=.335$. Participants in the eye movement condition in Experiment 1 also recalled more hits $(M=0.27$, $S D=0.09)$ than eye movement participants in Experiment $2(M=0.19, S D=0.11), F(1,136)=$ $11.62, p=.001, \eta_{\text {partial }}^{2}=.079$. All other interactions were non-significant $\left(F_{S}<3.74, p s>.055\right)$.

False Recall. A 2 (Condition: Eye Movements vs. Control) $\times 2$ (Valence: Negative vs. Neutral) $\times 2$ (Time: Immediate vs. Delayed) repeated measures ANOVA was performed on false recall and a similar pattern was found. That is, no significant three-way interaction emerged, $F(1,136)=0.14, p=.71$. A statistically significant interaction effect between condition 


\section{Chapter 6}

and time emerged, $F(1,136)=5.09, p=.026, \eta_{\text {partial }}^{2}=.036, \mathrm{BF}_{10}=0.09$. Simple effect analysis revealed a statistical significant simple main effect of time, but only for the control condition. Participants in the control condition of Experiment $1(M=0.29, S D=0.17)$ had higher false recall levels than control participants of Experiment $2(M=0.19, S D=0.14 ; F(1,136)=7.35, p=.008$, $\left.\eta_{\text {partial }}^{2}=.512\right)$. All other interactions were non-significant $(F s<1.90, p s>.171)$.

Intrusions. A 2 (Condition: Eye Movements vs. Control) × 2 (Time: Immediate vs. Delayed) ANOVA performed on uncorrected number of intrusions yielded no statistically significant interaction, $F(1,136)=0.15, p=.700$. No main effect of condition was found, $F(1,136)$ $=1.57, p=.212$. A significant main effect of time was found, $F(1,136)=13.09, p<.001, \eta^{2}$ partial $=.088, \mathrm{BF}_{10}=7.94$. That is, when memory was immediately tested (Experiment 1 ), fewer intrusions were recalled $(M=1.50, S D=0.28)$ than when memory was tested after a delay $(M$ $=2.91, S D=0.29$; Experiment 2).

\section{Recognition}

Hit Rates. A 2 (Condition: Eye Movements vs. Control) × 2 (Valence: Negative vs. Neutral) $\times 2$ (Time: Immediate vs. Delayed) repeated measures ANOVA performed on hits yielded a nonsignificant three-way interaction, $F(1,136)=0.67, p=.41$. A statistically significant interaction effect emerged between condition and time, $F(1,136)=5.14, p=.025, \eta^{2}$ partial $=.036, \mathrm{BF}_{10}=6.88$. Simple effect analysis revealed a simple main effect of time for both conditions. That is, control participants in Experiment 1 had more hits $(M=0.83, S D=0.09)$ than control participants in Experiment $2\left(M=0.64, S D=0.16 ; F(1,136)=39.35, p<.001, \eta_{\text {partial }}^{2}=.224\right)$. Eye movement participants in Experiment 1 had more hits $(M=0.78, S D=0.12)$ than eye movement participants in Experiment $2\left(M=0.69, S D=0.13 ; F(1,136)=9.96, p<.002, \eta_{\text {partial }}^{2}=.068\right)$. The simple main effect of condition was statistically non-significant ( $p s>.13$ ). All other interactions were nonsignificant $(F s<3.11$, ps $>$.080).

False Recognition. A 2 (Condition: Eye Movements vs. Control) $\times 2$ (Valence: Negative vs. Neutral) $\times 2$ (Time: Immediate vs. Delayed) repeated measures ANOVA performed on false recognitions yielded no statistically significant interactions ( $F_{S}<3.58, p s>.060$ ). A main effect of valence was found, $F(1,136)=12.39, p<.001, \eta_{\text {partial }}^{2}=.083, \mathrm{BF}_{10}=29.04$. More negative words $(M=0.75, S D=0.25)$ were recalled compared with neutral words $(M=0.67, S D=0.27)$. 


\section{DISCUSSION}

The overall pattern observed in Experiment 2 was that after a delay of 48 hours, more false memories for both recall and recognition were found in the eye movement condition than in the control condition. This effect even persisted when we looked at the corrected recognition scores (see Exploratory Analyses). Interestingly, we also found that the eye movement condition had overall higher correct memory levels than the control condition, an effect that has previously been observed by Nieuwenhuis and colleagues (2013). In line with Shah and Knott (2018), participants who performed eye movements made more remember responses than control participants.

\section{GENERAL DISCUSSION}

EMDR aims to change the quality of autobiographical memories of patients who suffer from aversive memories. Understandably, much of the literature on EMDR has understandably focused on its positive effects (i.e., reductions in vividness and emotionality; see e.g., van den Hout et al., 2014). However, researchers have recently begun to address unintended side effects of psychotherapy (e.g., Rozental et al., 2016) and one of these are false memories. During EMDR, parts of the traumatic memory will be retrieved until the vividness and emotionality linked to the traumatic memory sufficiently declined (van den Hout \& Engelhard, 2012). However, each time a memory is retrieved, memory is reconstructed and during such reconstruction, false memories may arise (McNally, 2005). The current experiments examined whether eye movements, as used in EMDR, might enhance the production of spontaneous false memories.

The main results can be summarized as follows. First, when participants had to perform eye movements during memory retrieval immediately after the encoding of stimuli (Experiment 1), eye movements did not significantly increase correct memory or false memory rates. Second, when memory was tested after a 48 hours delay (Experiment 2), eye movement participants had higher correct and false memory rates than control participants. In line with Nieuwenhuis et al. (2013), we found in Experiment 2 that participants who performed eye movements recalled and recognized more presented words (i.e., correct memories) than control participants. Importantly, even when we corrected for this enhanced memory effect in Experiment 2, eye movements were still associated with higher false memory levels. 


\section{Chapter 6}

Third, we found that eye movement participants (Experiment 2) recalled more correct neutral items than control participants. This is interesting, as the working memory account would lead one to predict that eye movements reduce the vividness and emotionality of both neutral and traumatic memories, and as a result, both types of memories will become less salient. However, we, and others (e.g., van den Hout, Eidhof, Verboom, Littel, \& Engelhard, 2014; but see Littel, Remijn et al., 2017) noted that eye movements do not undermine the quality of neutral memories. This finding requires follow up research, because at face value it is difficult to reconcile with the working memory account.

Fourth, in line with Shah and Knott (2018), we found that eye movement participants made more remember responses for critical lures than control participants (Experiment 2). One distinct possibility if that performing eye movements, may make it more difficult for participants to accurately discriminate between true and false items (Mather, Henkel, \& Johnson, 1997). As a result, false items might be evaluated more often as remembered in the eye movement than in the control condition.

One prominent framework for understanding spontaneous false memories is FTT (Brainerd \& Reyna, 2002). FTT posits that memories are sustained by two independent memory traces: verbatim (i.e., item-specific characteristics of an experience) and gist (i.e., general meaning of the experience) traces. One of the main tenets of FTT is verbatim traces will disintegrate more rapidly than gist traces, causing individuals to rely over time more on the general meaning of events (i.e., gist traces). FTT assumes that false memories are the result of overreliance on gist traces when verbatim traces have become inaccessible. To the extent that performing eye movements make memories less salient, FTT would predict that it encourages individuals to rely on the gist of experiences, thereby promoting false memories. However, FTT also postulates than when correct memory increases, reliance on verbatim traces increases as well, thereby lowering false memory rates (Brainerd \& Reyna, 2002). Our findings are difficult to reconcile with this perspective. That is, in Experiment 2, we found that eye movements are associated with both elevated correct memory levels and increased false memories. Also, although control participants of Experiment 2 recalled and recognized fewer hits than control participants in Experiment 1, the two control groups did not differ with regard to false recall and recognition, a pattern that runs counter to what FTT would predict.

An alternative framework is the Associative-Activation Theory (AAT; Howe et al., 2009), which specifies that during the encoding of an event, spreading activation will occur. This spreading activation connects the event to associated theme nodes in an individual's 
knowledge base (e.g., memories of going to a beach or a park are stored under the general topic of "going out"). Because of this spreading activation, concepts might become activated that were not part of, but only related to, the encoded event. The activation of such notexperienced concept can lead to the creation of false memories. AAT predicts that an increase in correct memories goes along with an increase in false memories (Howe et al., 2009). By implication, when people retrieve more correct memories due to eye movements, more spreading activation will occur. As a result, the chance that associated, but not experienced, concepts will be activated increases and this will promote false memories. The fact that we found a significant correlation between true and false recall in Experiment $2(r(34)=0.395$, $p=.021)$ supports such an interpretation.

The current experiments have some important limitations. First, the DRM paradigm has been criticized for its lack of ecological validity (for an overview see Wade et al., 2007; but see Otgaar et al., 2017). For example, Patihis, Frenda and Loftus (2018) showed that there is no relationship between endorsement of lures in the DRM paradigm and autobiographical false memories. The DRM paradigm generally does not involve autobiographical memories, whereas autobiographical memories are usually the primary focus of EMDR therapists. Indeed, the results obtained with the DRM paradigm in the current experiments are only a first step to examine the susceptibility to spontaneous false memories after performing eye movements. By using virtual reality techniques (i.e., experience real life situations within a simulation setting) to create autobiographical material, future research could examine false memory proneness after performing eye movements during retrieval of autobiographical memories.

A second limitation is that we employed the EMDR kit for our eye movement manipulation. In other research (e.g., van den Hout et al., 2014), a computerized eye movement task was used and it might well be the case that this technique induces more reliable eye movements. On the other hand, the kit is used in clinical practice (https://www.emdrkit.com) and hence, our results might be informative for what might occur during therapeutic sessions.

Third, the a priori power analysis was based on effect sizes observed in previous work on false memories (Howe et al., 2009). However, Morey and Lakens (2016) argued that basing the power of new studies on effect sizes from previous studies is not ideal, as one is indirectly selecting insufficient power for the study at hand. As the Bayes Factor depends on sample size (De Santis, 2007), the low power probability of statistical tests in the current experiments might explain the anecdotal Bayes Factors (i.e., BF < 1) for the critical main effects in Experiment 1. 


\section{Chapter 6}

Some researchers observed that performing eye movements during memory recall increases individuals' susceptibility to misinformation that is offered by others, which is one prominent source of false memories (Houben et al., 2018; but see Calvillo \& Emami, 2019; van Schie \& Leer, 2019). Our results indicate that eye movements might increase both correct memories and spontaneous false memories as well. Of course, this finding needs replication and its boundaries and generalizability need to be examined. However, given this state of affairs, EMDR therapists are well advised to take potential false memory effects of their intervention into account. 



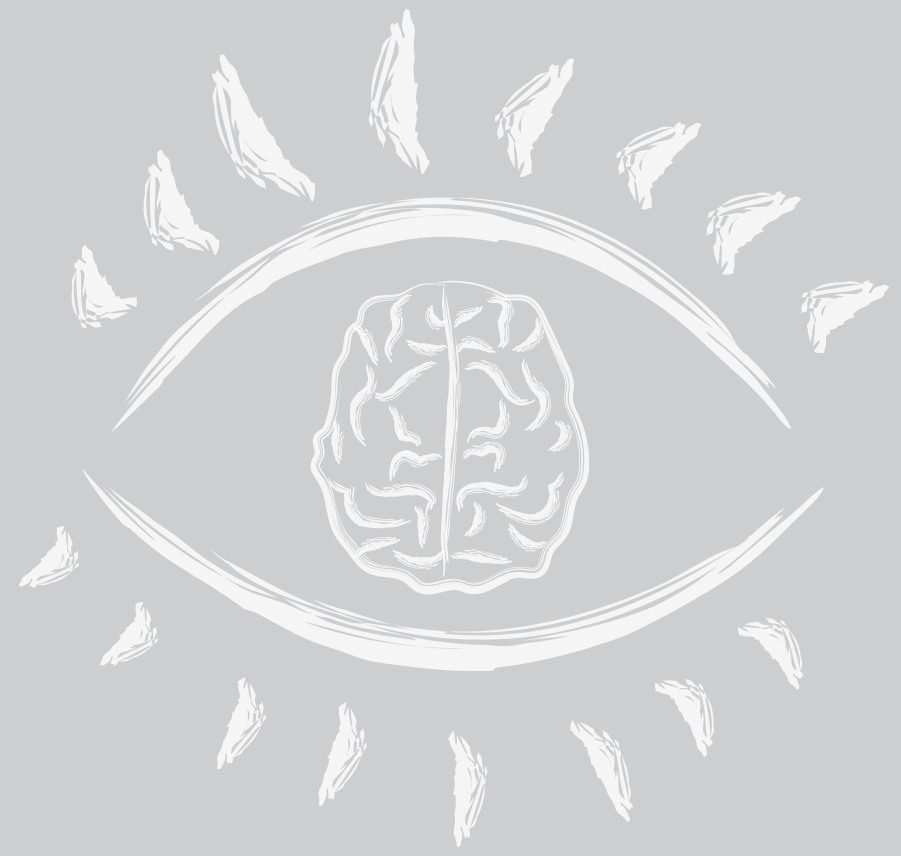




\section{CHAPTER 7}

\section{General Discussion}

This chapter is an extended and combined version of the following publications:

Houben, S. T. L., Otgaar, H., Rassin, E., \& Merckelbach, H. (2020). Getuigen die Eye Movement Desensitization and Reprocessing (EMDR) ondergaan: Een riskante osmose tussen psychologie en recht [Witnesses undergoing Eye Movement Desensitization and Reprocessing (EMDR): A risky osmosis between psychology and law]. Expertise en Recht, 4, 119-123.

Houben, S. T. L. (2020). Tinder voor psychotherapie? Over de blinde vlek van therapeuten [Tinder for psychotherapy? About the blind spot of therapists]. De Psycholoog, 55, 10-18. Merckelbach, H., Houben, S. T. L., Dandachi-FitzGerald, B., Otgaar, H., \& Roelofs, J. (2018). Als psychotherapie faalt [When psychotherapy fails]. De Psycholoog, 10, 10-21. 



\section{Aims of this Dissertation}

This dissertation examined the false memory potential of Eye Movement Desensitization and Reprocessing (EMDR). Several studies were conducted to provide a complete overview of factors that could be involved in creating such potential. Section I (Chapters 2 and 3) focused on general aspects of the EMDR protocol (i.e., EMDR instructions), while Section II (Chapters 4, 5, and 6) focused on EMDR therapists' beliefs about memory and the link between eye movements as used in EMDR and false memory production. In this General Discussion, an overview of the key findings is presented. Following this, conclusions will be drawn about the memorial consequences of EMDR and practical and theoretical reflections concerning EMDR administration will be elaborated as well.

\section{General Aspects of the Dutch EMDR protocol}

Section I primarily focused on the instructions of the Dutch protocol of EMDR. EMDR protocols per country differ from Shapiro's original EMDR protocol. The Dutch EMDR protocol differs in several ways from the original protocol developed by Shapiro. One aspect that differs is the manner in which the target image is identified. Dutch EMDR practitioners can chose between two metaphorical instructions: a video or photobook metaphor. These instructions, however, are well-known psychological myths as our memory does not operate as a video camera or as a photobook. The use of such instructions in a therapeutic intervention is questionable, as it may lead to incorrect inferences about how memory is supposed to operate. Chapter $\mathbf{2}$ examined how participants evaluated the metaphoric instructions used to identify the target image. Participants received the video, photo or control instruction and were asked to indicate how vivid and emotional they expected to recall the traumatic memory, how credible they found the instruction, and how reliable they would evaluate a therapist providing such an instruction. Especially participants who received the video metaphor expected to recall a memory more emotional and vivid as compared with the photobook metaphor and control instruction. No statistical difference on emotional and vividness between the photobook metaphor and control instruction was found. Though a highly emotional and vivid memory is beneficial for the desensitization phase in EMDR, this expectancy effect may lead to therapeutic demand characteristics (Kanter et al., 2002). Patients could reason to report as many details as possible, irrespective of whether they are correct.

Relatedly, Littel et al. (2017) did not find robust expectancy effects for prior knowledge about EMDR (i.e., that it reduces the vividness and emotionality of traumatic memories; 


\section{Chapter 7}

phase 4 - 6; see also Mertens, van Schie, Lammertink, Littel, \& Engelhard, 2020)7. However, the instructions examined in Chapter 2 are embedded in phase $1-3$, and an expectancy effect during these initial phases might persist throughout the remaining phases of the protocol (see Appendix 1).

Regarding the credibility of the instructions, all participants indicated they found the instructions to be confusing. Furthermore, they would not regard a therapist to be reliable when such an instruction was provided. The quality of the therapeutic relationship (i.e., therapeutic alliance) is established during the start of the treatment and is predictive of symptom reduction (Reefhuis et al., 2019). If patients find their therapist to be unreliable, it could harm the therapeutic relationship and treatment outcome.

Although performing eye movements is a central component of EMDR, several alternative dual tasks are used as well during EMDR therapy. In Chapter 3, a meta-analysis was performed on the effect of eye movements and alternative dual tasks on the vividness and emotionality of negative autobiographical memories in laboratory studies. Eye movements and alternative dual tasks exerted similar effects on vividness and emotionality ratings. This effect was stronger for vividness ratings than for emotionality ratings. This is interesting, because EMDR's main aim is to decrease the emotionality of negative autobiographical memories (Shapiro, 2002). However, Lee and Cuijpers (2013) found similar results (i.e., stronger effect for vividness than emotionality). Even more so, in Smeets and colleagues' (2012) study, vividness ratings dropped more quickly (i.e., 2 s) than emotionality (i.e., 74 s), hinting to the possibility that emotionality ratings start to decrease only when vividness has decreased. Hence, vividness might play a larger role when applying eye movements than initially assumed.

Other meta-analyses showed that EMDR is more effective in treating PTSD when compared with waitlist controls and equal or more effective than treatment as usual (i.e., cognitive behavioral therapy; see e.g., Chen, Zhang, Hu, \& Liang, 2015; Chen et al., 2018; Cuijpers et al., 2020; Davidson \& Parker, 2001; Diehle, Opmeer, Boer, Mannarino, \& Lindauer, 2015; Field \& Cottrell, 2011; Lee \& Cuijpers, 2013; Lewey et al., 2018; Moreno-Alcázar et al., 2017; Rodenburg, Benjamin, de Roos, Meijer \& Stams, 2009; Wilson et al., 2018). However, a more recent meta-analysis indicated that the included studies contained high risk of bias (i.e., in methodology, results, or inferences) and had considerable heterogeneity (Cuijpers et al., 2020). When only studies with low risk of bias were included, the number of included studies dropped and no statistically significant difference between EMDR's and other therapies' effectiveness for PTSD emerged (Cuijpers et al., 2020). Even more so, Cuijpers and colleagues 
found considerable indications for researcher allegiance (i.e., the belief in superiority of a treatment).

Though these few meta-analyses suggest that much is unknown about EMDR's effectiveness in treating PTSD, EMDR has already been applied to treat other mental disorders in practice, for example eating disorders (Bloomgarden \& Calogero, 2008; Dziegielewski \& Wolfe, 2000; Halvgaard, 2015; Hudson et al., 1997; Zaccagnino, Cussino, Callerame, Civilotti \& Fernandez, 2017), medically unexplained symptoms (Brown, McGoldrick, \& Buchanan, 1997; Chemali \& Meadows, 2004; Grant, 2000; Grant \& Threlfo, 2002; Gupta \& Gupta, 2002; Kelley \& Benbadis, 2007; Mazolla et al., 2009; McGoldrick, Begum, \& Brown, 2008; Ray \& Page, 2002; Silver, Rogers, \& Russell, 2008; Royle, 2008; Russell, 2008a, 2008b; de Roos et al., 2010; Wilensky, 2006), mood disorders (Behnammoghandam, Alamdari, Behnammoghadam, \& Darban, 2015; Behnammoghadam, Behnammoghadam, \& Salehian, 2015; Hase et al., 2015; Hofmann et al., 2014; Hogan, 2001; Lei \& Zhen-Yin, 2007; Muana Gauhar, 2016; Novo et al., 2014; Tang, Yang, Yen, \& Lui, 2015), chronic pain (Allen, 2004; de Roos et al., 2010; Estergard, 2009; Friedberg, 2004; Gerhardt, 2016; Grant \& Threlfo, 2002; Hassard, 1995; Kavakci, Semiz, Kaptanoglu, \& Ozer, 2012; Konuk, Expözdemir, Atceken, Aydin, \& Yurtsever, 2011; Marcus, 2008; Mazzola et al., 2009; Schneider, Hofman, Rost, \& Shapiro, 2008; Wilensky, 2006), anxiety disorders (Bauman \& Melnyk, 1994; Cook-Vienot \& Taylor, 2012; Doering, Ohlmeier, de Jongh, Hofmann, \& Bisping, 2013; Feske \& Goldstein, 1997; Foley \& Spates, 1995; Goldstein, de Beurs, Chambbless, \& Wilson, 2000; Gosselin \& Matthews, 1995; Horst et al., 2017; Littel et al., 2017; Muris, Merckelbach, Holdrinet, \& Sijsenaar, 1998; Passoni et al., 2018; Rahimi et al., 2018; Rathschlag et al., 2014; Staring et al., 2016; Triscari, Faraci, Catalisano, D’Angelo, \& Urso, 2015; Zeighami, Behnammoghadam, Moradi, \& Bashti, 2018), substance use disorders (Hase, Schallmayer, \& Sack, 2008; Perez-Dandieu, \& Tapia, 2014), psychotic disorders (van den Berg et al., 2015; de Bont et al., 2013, 2016; Kim et al., 2010; van Minnen et al., 2016), obsessive compulsive disorder (Nazari, Momeni, Jariani, \& Tarrahi, 2011), and conduct disorder (Soberman, Greenwald, \& Rule, 2002).

Though this overview might seem like a substantial number of research studies, meta-analyses on the use of EMDR to treat these mental health disorders are sceptical about its efficacy (Balbo, Zaccagnino, Cussino, \& Civilotti, 2017; Carletto et al., 2017; Cuijpers et al., 2020; van Rood \& de Roos, 2009; Tesarz et al., 2014; Yunitri et al., 2020), mainly due to the low quality of studies (Cuijpers et al., 2020). A major shortcoming of the meta-analyses, including the meta-analysis of Chapter 3 , is that only a limited number of studies can be included due 


\section{Chapter 7}

to, for example, in- and exclusion criteria and missing data (Balbo et al., 2017; Carletto et al., 2017; Chen et al., 2015; Chen et al., 2018; Field \& Cottrell, 2011; Houben et al., 2020; Lee \& Cuijpers, 2013; Moreno-Alcázar et al., 2017; Rodenburg et al., 2009; van Rood \& de Roos, 2009; Tesarz et al., 2014; Valiente-Gómez et al., 2017; Wilson et al., 2018; Yunitri et al., 2020; but see Cuijpers et al., 2020; Davidson \& Parker, 2001; Landin-Romero et al., 2018; Lewey et al., 2018). Though the inclusion and exclusion criteria differed per meta-analysis, overall, meta-analyses found small effect sizes for EMDR's efficacy (e.g., $g=0.36$, Cuijpers et al., 2020), and due to the high heterogeneity (i.e., $l^{2}>75 \%$; Higgins \& Thompson, 2002) and high risk of bias in all meta-analyses (e.g., Cuijpers et al., 2020; Houben et al., 2020) conclusions about the beneficial effects of EMDR should be taken cautiously.

The results from Cuijpers et al. (2020) indicate that EMDR may be effective to treat PTSD in the short-term, but EMDR is not validated enough by empirical studies to treat other mental health disorders. Moreover, there is a lack of studies examining long-term effects of EMDR. Without high quality studies that are sufficiently powered and examine long-term effects, EMDR's efficacy remains uncertain. John-Baptiste Bastien, Jongsma, Kabadayi, and Billings (2020) found a stronger effect for EMDR (Cohen's $d=-0.46$ ) compared with trauma focused-cognitive behavior therapy (TF-CBT; Cohen's $d=-0.30$ ), but still recommend to offer TF-CBT to patients as it is more evidence based. Clearly, research on EMDR is still in its infancy.

\section{Beliefs about Memory}

If there is one topic that illustrates the gap between scientists and practitioners, it is the debate concerning the authenticity of recovered memories (Dalenberg, Brand, Loewenstein, Frewen, \& Spiegel, 2020; Patihis, Otgaar, \& Merckelbach, 2019). Due to the considerable number of patients claiming to have recovered memories in the 1990s, it became clear that, amongst others, therapists hold strong beliefs in the concept of repressed memories and applied suggestive therapeutic techniques (Ceci \& Loftus, 1994). Up to date, no scientific evidence exists to confirm the existence of repressed memories (for a review see Otgaar, Howe, Patihis et al., 2019). More specifically, researchers have sometimes found that individuals who claim to not remember the traumatic event, actually mentioned the event to others (Benner \& Lindsay, 2007; Merckelbach et al., 2005; Raymaekers et al., 2011; Schooler, Ambadar, \& Bendiksen, 1997). This underestimation of prior remembering can contribute to the experience of being amnesic for the traumatic event (Raymaekers et al., 2011).

Because EMDR targets traumatic autobiographical memories, it is imperative that 
EMDR therapists have sufficient knowledge about the functioning of memory and are aware of controversial ideas about how memory operates. This way, they can (1) apply this knowledge in practice and thereby optimising their own expertise and (2) inform a patient adequately about

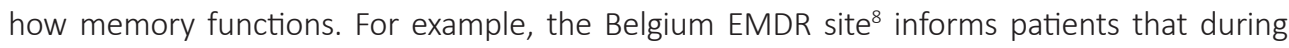
a traumatic event the mind is unable to process the event and that the traumatic memory remains in the body. However, scientific literature has shown that traumatic events are well remembered, and do not reside in bodily memories (Vervaeke et al., 2002). It is inappropriate to provide patients with unsubstantiated ideas concerning the functioning of memory and in this way create expectancies that may backfire.

In Chapter 4, memory beliefs in EMDR practitioners were examined. In two surveys, EMDR therapists, students, and researchers provided their beliefs about correct and controversial statements about the functioning of memory. In Study 1 of Chapter 4, students and EMDR practitioners were provided with a case vignette in which a patient recovered memories of sexual abuse after an EMDR session. A majority in both groups (60\% and 75\%, respectively) indicated that the recovered memory was likely to be authentic. As for the memory beliefs, though EMDR practitioners had adequate knowledge of several memory aspects (e.g., they know memory does not operate as a video camera; $97 \%$ of EMDR therapists, $87 \%$ of students and $97 \%$ of researchers; Cramer's $V=.16)$, they nevertheless strongly believed in the concept of unconscious repression (70\%, 87\%, and 66\%, respectively; Cramer's V = .19). Brewin et al. (2019) suggested that surveys as the one in Chapter 4 examine the belief in conscious repression (i.e., repression), and that this topic is not a controversial one. However, in Chapter 4, we explicitly asked for unconscious repression. Otgaar, Wang, and colleagues (2019) found that the belief in unconscious repression is still widely accepted. It is problematic when EMDR practitioners (strongly) believe in these controversial topics. EMDR aims to alter traumatic autobiographical memories. It is therefore important that EMDR practitioners are knowledgeable about the functioning of memory. Though it remains unknown whether a therapist who endorses such controversial beliefs actually acts upon this knowledge, GoreFelton and colleagues (2000) observed that personal beliefs can influence professional judgments. Strongly believing in the concept of repressed memories might lead to backward reasoning (i.e., "vague symptoms might be a sign of a repressed memory"). Consequently, therapists might (suggestively) seek for these repressed memories, thereby increasing the likelihood of false memory formation.

The results of Chapter 4 are in line with previous studies on memory beliefs (Akhtar

${ }^{8}$ See https://emdr-belgium.be/informatie-over-emdr/traumas-leren-verwerken-met-emdr 


\section{Chapter 7}

et al., 2018; Brewin et al., 2019; Dammeyer et al., 1997; Dodier, Melinder, Otgaar, Payouw, \& Magnussen, 2019; Golding et al., 1996; Gore-Felton et al., 2000; Magnussen \& Melinder, 2012; Ost et al., 2013; Otgaar, Wang et al., 2019; Patihis et al., 2014; 2018; Yapko, 1994). In general, based on the recent surveys examining memory beliefs, it can be concluded that the belief in the controversial topic of repressed memory is very much alive. Clinicians are in close contact with, amongst others, eyewitnesses and victims. It is during these interactions that memory of these groups can become contaminated. The belief in repression can lead therapists to (unintentionally) seek for repressed memories. Consequently, patients could form false memories (or beliefs) and conclude that an alleged event might have happened. Setting the consequences of this aside, it is unprofessional to educate one's patient with unsubstantiated information about the functioning of (traumatic) memory. Though it is hard for therapists to improve their expertise (Tracey et al., 2014), educating oneself to stay up-to-date with scientific knowledge should not be. However, it is unknown to which degree therapists who strongly believe in the concept of repressed memories also act upon this belief.

\section{Eye Movements and False Memories}

Section II primarily focused on the effect of eye movements on the susceptibility to suggestive (Chapter 5) and spontaneous (Chapter 6) false memories. The results of Chapter 4 indicated that EMDR therapists still hold dubious beliefs about the functioning of memory. Based on these beliefs, one might expect such therapists to ask suggestive questions during the therapy session or even suggest a complete event (Ceci \& Loftus, 1994). In Chapter 5, I examined the effect of eye movements on the susceptibility to suggestive false memories. Participants saw a video depicting a car crash and either performed eye movements or not afterwards. Next, participants received misinformation concerning the car crash and completed a recognition test. Participants who performed eye movements more often endorsed the misinformation than control participants. This finding indicates that individuals might become more vulnerable to accept misinformation after performing eye movements compared with not performing eye movements. Another interesting finding was that declines in subjective vividness and emotionality did not statistically differ between performing eye movements or not. Though the effect was stronger for eye movement participants, no statistical difference of these subjective measures between both conditions could be found in Chapter 5 . This finding is in contrast to the robust finding that participants who performed eye movements show a stronger decrease in vividness and emotionality than participants who did not perform eye 
movements (van den Hout \& Engelhard, 2012).

Lee, de Jongh, and Hase (2018) provided a critical commentary to the findings reported in Chapter 5. According to Lee et al., the study reported in Chapter 5 ignored previous research that is incompatible with our findings. Indeed, the findings of Chapter 5 contradict the finding from an earlier study of Parker et al. (2009). Parker and colleagues (2009) showed that performing eye movements led to increased true memory rates and a decrease of the misinformation effect. An important side note was that the experimental designs differed on a critical dimension between studies (Houben, Otgaar, Roelofs, \& Merckelbach, 2019). Participants in the Parker study had to perform eye movements after the misinformation was provided. Following imagination deflation, this would lead to a degradation of the misinformation (i.e., misinformation become less vivid). This procedure is less efficient to examine the misinformation effect because eye movements make the misinformation vaguer and thereby harder to incorporate into one's memory. In Chapter 5, I chose to degrade the original memory (i.e., perform eye movements after observing the traumatic video). As a result, the initial memory will become vaguer. These are ideal circumstances for a participant to endorse misinformation. In addition, this sequence is more in line with what happens in real life and what expert witnesses sometimes encounter in the courtroom (Shaw \& Vredeveldt, 2019). For example, a patient has a vague memory of a traumatic incident, receives treatment and is then (unintentionally) provided with misinformation. The design used by Parker et al. is not applicable to such cases.

A second critical point of Lee et al. (2018) was that the experiment described in Chapter 5 failed to take into account contextual differences (i.e., laboratory versus clinical settings). Lee and colleagues stated that EMDR practitioners are trained to refrain from suggesting information. Though this might be taught during training, it is not guaranteed that therapists stick to what they learned. As evident from Chapter 3 (see also Phelps \& Hofmann, 2019), EMDR therapists continue to believe in the controversial concept of repressed memories and they could, suggestively, seek for repressed memories potentially leading to false memory creation. In addition, Lee et al. explicitly mentioned that EMDR takes advantage of the reconstructive aspect of memory, which is prone to error and distortion. This reconstruction would help individuals to better deal with future events (Finnie \& Nader, 2012). Although the authors are aware that the intervention they advocate can lead to error and distortions, they do not seem to see the importance and relevance of experimental studies on the impact of eye movements on false memory formation. Potential harmful effects of psychological 


\section{Chapter 7}

interventions have not been well examined (Jonsson, Alaie, Parling, \& Arnberg, 2014) and experimental studies are a first step to fill this knowledge gap.

Because it is unknown how eye movements lead to changes in PTSD symptomatology, it is unclear why there is a mismatch between experimental and clinical research (Kredlow, de Voogd, \& Phelps, 2020). Laboratory studies allow for a more controlled environment, specific research questions can be examined in a shorter time period, and, maybe most importantly, can be examined in healthy controls before testing actual patients (Kredlow et al., 2020). It is striking that experimental studies that might uncover potential limitations of an effective treatment are criticized, while experimental studies on the same treatment that underline its' effectiveness are acknowledged. But, perhaps, EMDR therapists suffer from confirmation bias (i.e., laboratory studies are acknowledged when they show support for EMDR's efficacy, but are not accepted when possible shortcomings are revealed).

Two attempts to replicate the study in Chapter 5, however, did not find similar results (Calvillo \& Emami, 2019; van Schie \& Leer, 2019; see Table 7.1). All three studies failed to find an effect of eye movements on vividness and emotionality ratings, which contradicts the usual pattern that emerges in eye movement research. However, this dissimilarity could be due to the difference in materials (i.e., no autobiographical memories; Leer, Engelhard, Dibbets, \& van den Hout, 2013; van Schie, Engelhard, \& van den Hout, 2015; but see Leer et al., 2017). More importantly, neither Calvillo and Emami (2019), nor van Schie and Leer (2019) observed the misinformation effect as reported in Chapter 5. Several explanations for these failed replications can be offered: the finding in Chapter 5 might be a false positive, the other two studies might be false negatives, there are differences in participants (e.g., personal motivation, gender) between studies, and observer expectancy effect (Rosenthal, Kohn, Greenfield, \& Carota, 1966) might play a role. This latter explanation can be solved by using methods that do not rely on external pressure (e.g., DRM paradigm). Apart from the question whether eye movements truly promote misinformation effects, memory remains reconstructive and misinformation effects due to suggestion can also occur, especially if therapists are unaware of the science of memory. This latter issue is pertinent and can, as will be discussed below, have immense consequences. 
Table 7.1 Overview of Correct and Endorsed Misinformation Answers and Effect Sizes per Study.

\begin{tabular}{|c|c|c|c|c|c|c|c|c|c|}
\hline & \multicolumn{5}{|c|}{ Correct Answers } & \multicolumn{4}{|c|}{ Misinformation Answers } \\
\hline & $N$ & EM & Control & Cohen's d & $95 \% \mathrm{Cl}$ & EM & Control & Cohen's d & $95 \% \mathrm{Cl}$ \\
\hline Chapter 5 & 82 & 12.46 & 13.51 & -0.88 & {$[-1.34,-0.43]$} & 2.0 & 1.15 & 0.77 & {$[0,27,1.16]$} \\
\hline $\begin{array}{l}\text { van Schie \& } \\
\text { Leer (2019) }\end{array}$ & 206 & 13.20 & 13.42 & -0.17 & {$[-0.44,0.11]$} & 0.9 & 0.85 & 0.06 & {$[-0.21,0.34]$} \\
\hline $\begin{array}{l}\text { Calvillo \& } \\
\text { Emami (2019) }\end{array}$ & 120 & 13.17 & 13.08 & 0.07 & {$[-0.28,0.43]$} & 1.08 & 1.1 & 0.02 & {$[-0.34,0.38]$} \\
\hline
\end{tabular}

Notes. $\mathrm{EM}=$ eye movement condition. $\mathrm{Cl}=$ Confidence Interval.

If an EMDR therapist refrains from suggestive questioning, it could still be the case that the treatment itself elicits false memories. Several studies have examined the effects of eye movements on spontaneous false memories (Christman et al., 2004; Parker \& Dagnall, 2007). Overall, these studies found that eye movements resulted in decreased (spontaneous) false recall compared with performing no eye movements. However, the procedure followed in these studies is incompatible with standard EMDR procedure (i.e., performing eye movements and memory recall simultaneous/y). Chapter $\mathbf{6}$ focused on spontaneous false memories and applied the standard EMDR procedure. In two experiments, participants viewed DRM word lists containing negative and neutral associated words. They were given a free recall and recognition test immediately after this (Experiment 1) or 48 hours later (Experiment 2). When memory was tested 48 hours later, eye movement participants showed higher true and false memory ratings. In line with Nieuwenhuis et al. (2013), participants who performed eye movements recalled more correct words than control participants did. In the current study, however, this was accompanied with an increase in false memories.

Previous research found that performing horizontal eye movements enhances episodic memory, an effect that was dubbed saccade-induced retrieval enhancement (SIRE; Christman et al., 2003; Lyle \& Martin, 2010; Lyle \& Edlin, 2015). The SIRE effect results in an increase in hit rates and a decrease in false alarm rates (i.e., false memories) in, for example, word lists (Nieuwenhuis et al., 2013) or pictures (Parker, Poole, \& Dagnall, 2020). The SIRE effect drew upon the idea of interhemispheric interaction as a possible explanation for the effect of eye movements as used in EMDR (Christman et al., 2003). In Chapter 6, participants who performed eye movements, indeed, recalled more correct memories, but also reported more false memories. Roberts, Fernandes, and MacLeod (2020), however, found weak evidence that eye movements would lead to memory enhancing effects (see also Matzke et al., 2015; Samara, Elzinga, Slagter, \& Nieuwenhuis, 2011). The inconsistency surrounding the 


\section{Chapter 7}

SIRE effect (i.e., only found in between subject designs or left-handedness) makes its use as a clinical tool questionable because its scientific reliability is low. Cognitive effects observed in laboratory settings must be reliable before they can be applied clinically. Hence, Roberts and colleagues (2020) emphasized the caution with which eye movements should be used as a clinical method.

Though the SIRE effect was especially prominent in the increase of true recall of neutral items in Chapter 6, this influence on neutral material is incompatible with the WM theory. According to the WM theory, neutral memories are not affected by eye movements. A possible explanation for the fact that eye movements do not influence neutral memories, would be that neutral material is not taxing WM as strongly as negative material does. Following the WM account, all emotional memories should lose their vividness and emotionality when performing a dual task. This means that if negative memories can become less negative, positive memories can become less positive (see van den Hout et al., 2001; Engelhard, van Uijen, \& van den Hout, 2010). An interesting side note, here, is that within the EMDR protocol, positive cognitions are installed by performing eye movements. Based on the results described above, this procedure seems ineffective. Turning to the valence of memories, neutral memories are not affected by the taxing of WM and remain highly vivid (van den Hout et al., 2014; but see Littel, Remijn, et al., 2017).

A limitation of Chapter 6 is its reliance on DRM word lists. The DRM paradigm does not include autobiographical material, which led some to argue that the results of Chapter 6 are not relevant for clinical practice (de Jongh \& Bicanic, 2020). Though its relevance for clinical practice is far stretched, the results do provide information about associations from memories that might, indeed, be elicited during EMDR therapy. For example, if a patient remembers other (details of) memories, a therapist could instruct to "park" these new memories and only work with the memories that are known at the start of treatment. In this way, it is prevented that the new, possibly false, memory will be elaborated, thereby increasing the belief in the erroneous memory. Though the practical relevance of research on false memory susceptibility after performing eye movements was questioned because of its materials, an important consideration is that in Chapter 6, instead of using the generally used E-prime task to elicit eye movements, an EMDR kit was employed. This tool is used in practice as well, meaning that in this respect the study in Chapter 6 narrowed the gap between laboratory and clinical studies (Kredlow et al., 2020).

De Jongh and Bicanic (2020) also stated that the experiment discussed in Chapter 
6 did not include a proper control condition. They argue that other interventions might also make individuals prone to false memory formation and that a condition receiving another intervention should have served as a control condition. Susceptibility to false memories is, indeed, not unique to EMDR, but following usual experimental standards, a proper control condition receives no intervention whatsoever.

Another important point to keep in mind is that Chapter 6 employed a different paradigm than Chapter 5. Chapter 5 specifically focused on external pressure (i.e., providing misinformation), while Chapter 6 focused on the intervention itself. The fact that van Schie and Leer (2019) failed to find a misinformation effect, does not mean that spontaneous false memories will be absent in an EMDR setting. Spontaneous and suggestion-induced false memories are simply two different concepts, which is important for clinicians to be aware of. Memory editing in clinical practice becomes more common (e.g., imagery rescripting; Phelps \& Hofmann, 2019), which might have harmful consequences when a patient decides to act upon these memories (e.g., enter the legal arena). An important note, however, is that false memories might be beneficial in some cases, and thus are not always harmful. For example, due to the false memory (either suggestive or spontaneous), a patient might be able to process the traumatic incident more efficiently. To compare it with another effective PTSD treatment, in imagery rescripting (Arntz, 2012), a therapist guides the patient to imagine a different course of events that is more acceptable to the patient's need (i.e., similar to cognitive reappraisal). In a way, misinformation is provided during imagery rescripting, but this helps the patient to process the trauma. False memories due to eye movements as used in EMDR could have similar effects.

\section{Theoretical frameworks}

Following the proposed theoretical framework in Chapter 1 (Figure 1.2), the SMF argues that vague memories would lead to higher difficulty differentiating between sources of information. More specifically, in Chapter 5 , it was argued that the endorsement of misinformation might be related to the decrease in vividness as a result of eye movements. Following the theoretical frameworks (i.e., FTT, SMF), eye movements make an experienced event less distinctive and, as a consequence, misinformation will be more easily accepted because (1) one would rely on gist traces (following $\mathrm{FTT}$ ) or (2) the discrepancy between the actual memory and the misinformation decreases and misinformation is more difficult to detect. However, as discussed above, two replication studies were not able to reproduce the results as described 


\section{Chapter 7}

in Chapter 5. An explanation might be that this relationship is mediated or moderated by other factors (e.g., fatigue after performing eye movements, speed of eye movements was too slow). Hence, for SMF (and FTT), the proposed theoretical framework for the relation between vividness and misinformation endorsement might be different from what was previously expected (see Figure 7.1). For example, fatigueness is a recurring effect of performing eye movements. It is possible that this factor influences the link between eye movements and false memories, because it impedes the memory retrieval process.

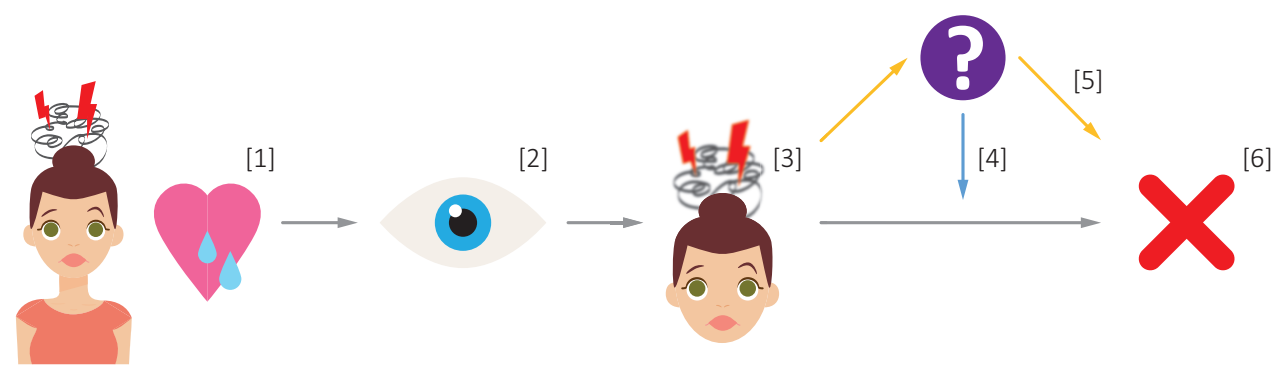

Figure 7.1 Adapted proposed theoretical framework on eye movements as used in EMDR and false memories (SMF and FTT). [1] An individual witnesses a traumatic event and experiences PTSD symptoms. [2] EMDR is the treatment of choice. [3] Due to the eye movements, the memory of the traumatic event becomes vaguer (i.e., less vivid and emotional). An unknown [4] moderator (blue line) or [5] mediator (orange line) variable might influence the effect of vague memories on [6] memory distortion. Following SMF or FTT, this will lead to decreased discrepancy detection or activation of gist traces, respectively.

Another explanation takes the link between vividness, emotionality and the memory itself as its starting point. According to the SMF (Lindsay \& Johnson, 1989), the relationship between vividness and endorsement of misinformation should be negative: highly vivid memory will lead to low endorsement of misinformation. A negative correlation between post vividness scores and endorsement of misinformation was found in Chapter 5, but not in Calvillo and Emami (2019), nor was there a correlation between vividness decreases and endorsement of misinformation in van Schie and Leer (2019; no correlation between post vividness scores and misinformation endorsement was performed). However, in Chapter 5, as well as in van Schie and Leer (2019) and Calvillo and Emami (2019), no statistical difference between the conditions with regard to vividness and emotionality decreases were found (see Figure 7.2). That is, all participants, irrespective of performing eye movements or not, showed decreases in vividness and emotionality. This is contrary to the general finding, namely that participants who perform eye movements exhibit stronger decreases in these measurements compared 
with the control condition (van den Hout \& Engelhard, 2012). However, even under the WM theory, it is still unclear how vividness and emotionality are related. For example, it is unknown whether both dimensions are equally affected by the taxing of the WM and how strongly both dimensions need to decrease. Smeets et al. (2012) reported that a reduction in vividness might lead to a decrease in emotionality (or vice versa, see Smeets et al., 2012). More research on the relationship between the vividness of memories and the misinformation effect is needed.

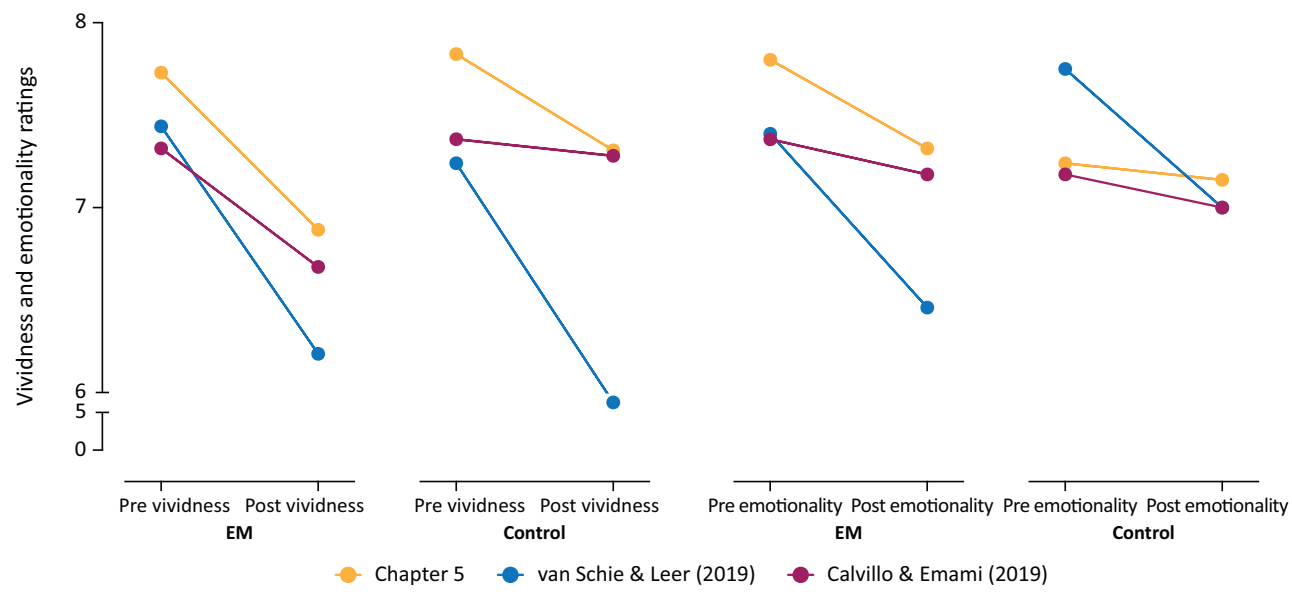

Figure 7.2 Overview of vividness and emotionality ratings in Chapter 5, van Schie and Leer (2019), and Calvillo and Emami (2019). Ratings ranged from $0=$ not vivid/emotional to $10=$ extremely vivid/emotional. $E M=$ eye movement condition.

As discussed above, the SIRE effect was considered to be a beneficial effect of performing eye movements because it increased true memory rates and decreased false memory rates. However, studies presenting the SIRE effect (e.g., Christman et al., 2013) were based on the idea of interhemispheric interaction as an explanation for the working mechanisms of EMDR. This explanation is, however, discarded by scientific studies (Gunter \& Bodner, 2008). The WM account states that the vividness and emotionality (e.g., quality) of memories is influenced by performing eye movements. The quantity (i.e., number of memories/details recalled) will not be affected by eye movements. Following the theoretical framework on spontaneous false memories as presented in Chapter 1 (Figure 1.2) and based on the results of Chapter 6, eye movements lead to an increase in true memory rates, which is accompanied by an increase in false memory rates as well. A statistically significant correlation between true and false recall was found in Experiment 2 of Chapter 6 ( $r(34)=0.395, p$ $=.021$ ). Following the AAT (Howe et al., 2009), true memories and related concepts, but 


\section{Chapter 7}

not presented, will be activated due to spreading activation. That is, an increase in correct memory rates amplified the likelihood of an incorrect, but related, memory to be recalled as well (see Figure 7.3). Hence, eye movements might influence the quality (i.e., vividness and emotionality) and the quantity: true memory rates might increase, but due to its connection with related but not presented nodes, there is a higher chance that false memories might be activated as well. This, then, contradicts the reasoning by the WM account. Hence, besides examining if memories become less vivid and emotional, it is important to know whether WM taxation impacts the number of details recalled as well. Such research is beneficial from a legal perspective (see below).

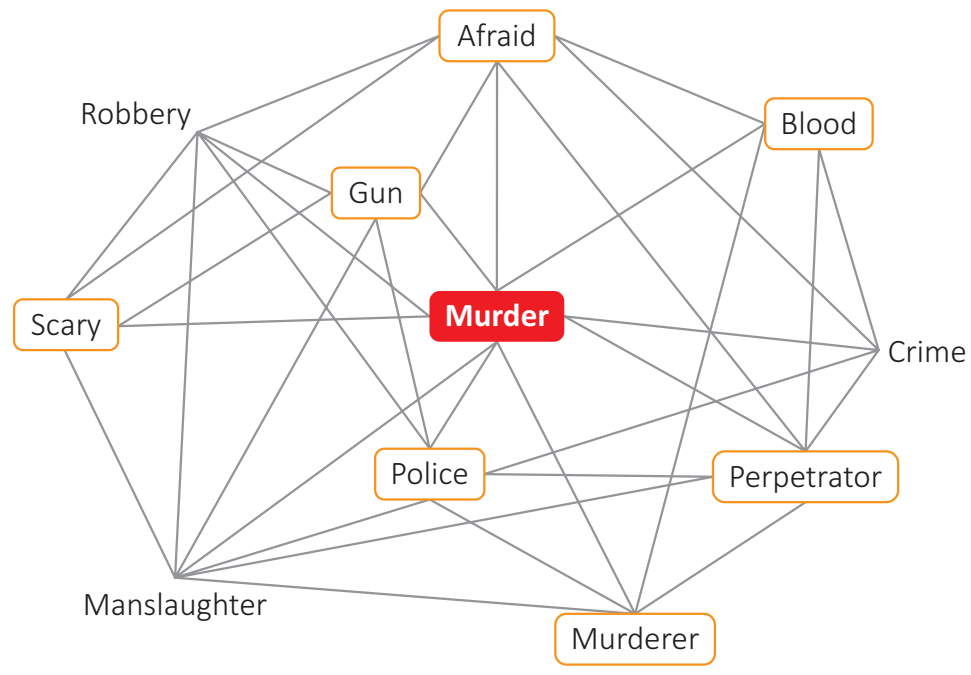

Figure 7.3 Example of a negative DRM word list. Following AAT, an increase in correct memories (orange circled words) increase the likelihood for a related, but false, memory (red circle) to be activated as well.

\section{Therapy Side Effects}

Although EMDR is beneficial for the majority of patients, a substantial number of patients do not show clinical improvement after EMDR treatment (10 - 73\% of patients who completed EMDR therapy did not improve; see Bradley, Greene, Russ, Dutra, \& Westen, 2005). These patients might experience side effects. One example of such an unwanted side effect is the emergence of new unpleasant memories (Rozental et al., 2016). More specifically, in the EMDR protocol (see Appendix 1), therapists are allowed to associate based on what a patient reports. Such associations may contribute to the formation of new unpleasant memories. 
Generally, however, knowledge about therapeutic side effects is scarce.

For medical treatment, a new drug is only prescribed when a preregistered randomized controlled trial (RCT) indicates its effectiveness and side effects have been documented. Patients then receive a prescription that includes when and how to use the medication and that also lists possible side effects (e.g., dizziness, headaches). However, acknowledgement of side effects among psychotherapists is not self-evident (Linden, 2013) as it is for medical treatments. Though RCTs in psychotherapy cannot completely follow the exact same procedure as in medicine (i.e., psychotherapy cannot be offered double blind), it would be naive to believe that psychotherapy does not have any side effects. For one thing, most RCTs on psychotherapy are not preregistered in the first place (Cybulski, Mayo-Wilson, \& Grant, 2016). Hence, possible therapeutic side effects might be underreported (Holmes et al., 2018; Parry, Crawford, \& Duggan, 2016).

RCTs in the psychotherapeutic domain typically fail to address side effects, but that in itself does not mean that such side effects are absent. Individual differences aside, on average, 13 to 18 psychotherapy sessions are needed to recover from mental health problems (Hansen, Lambert, \& Forman, 2002). However, seven to nine per cent of patients have been shown to deteriorate during therapy (Hannan et al., 2005; Hatfield, McCullough, Frantz, \& Krieger, 2010) and around five percent of the patients say to experience lasting bad effects from treatment (Crawford et al., 2016). Patients who reported to have experienced side effects also indicated that they were not informed sufficiently about the course of therapy. Indeed, most information leaflets for psychotherapy do not mention possible side effects, but how could they include such information when research on this topic is not lacking. Patihis and Pendergrast (2019) advocated to include information about potential hazards of recovered memories of abuse in the informed consent. However, as Loftus and Teitcher (2019) argued, such informed consent could be seen as a therapy risk, because it could lead to patients feeling reluctant to start treatment. Loftus and Teitcher furthermore argued that patients might not read an informed consent and research is needed on how to prevent harm in the first place.

An additional problem to the underreporting of therapeutic side effects is the lack of a consistent definition. Several taxonomies have been developed to help therapists in recognizing unwanted side effects (see Figure 7.4; Linden, 2013; Linden \& Schermuly-Haupt, 2014; Moritz et al., 2015; 2019). Side effects can occur due to external factors (e.g., being laid off and as a result an early termination of therapy), correctly performed interventions (e.g., increase in fear 


\section{Chapter 7}

during exposure; Parry et al., 2016) or incorrectly performed interventions (e.g., suggestive therapy eliciting false memories). Spontaneous false memories could be seen as an unwanted effect caused by correctly applied treatment, whilst suggestion-induced false memories are elicited due to an incorrectly applied treatment (e.g., by suggestive questioning). As depicted in Figure 7.4, this may lead to therapeutic failures. To optimally benefit from such taxonomies, it is of utmost importance that therapists are capable to monitor the therapeutic process. To illustrate, Hannan and colleagues (2005) found that for the patients who deteriorated during therapy, the therapists did not recognize such deteriorations. Linden (2013) has proposed a checklist to help therapists monitor possible side effects (Unwanted Event - Adverse Treatment Reactions; UE-ATR checklist; see Appendix 2). Rozental and colleagues (2016) created a checklist for patients, the Negative Event Questionnaire (NEQ; see Appendix 3).

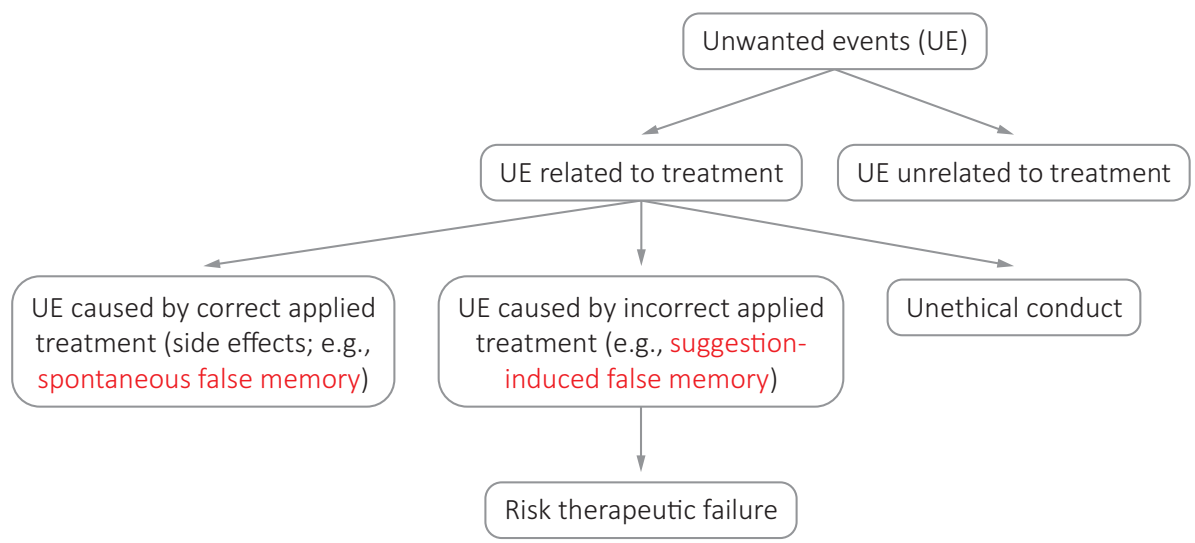

Figure 7.4 Proposed taxonomy of unwanted events of psychotherapy prosed by Linden (2013; UE = unwanted events).

As previously mentioned, therapists rarely address side effects. When they do report them, however, therapists oftentimes attribute the side effect to the patient (Gold \& Striker, 2011), refraining to look into their own role in the therapeutic process. A way to counter this lack of self-reflection (i.e., self-serving bias; attribute success to your own abilities, but ascribe failures to external factors) and to prevent "the blind leading the blind", is to use monitoring and feedback instruments. For clinical practice, it would be beneficial to use instruments that provide feedback from the patient and therapist perspective. The dual perspective on the therapeutic alliance is a strong predictor for treatment outcome (van Benthem et al., 2020). By adopting such dual perspective, therapists can obtain enough feedback and decide (in 
time) to alter the treatment plan. By using feedback, therapists can learn about possible side effects and bring their expertise to a higher level.

Several studies substantiate the use of feedback systems (Lambert, Harmon, Slade, Whipple, \& Hawkins, 2005; Janse, de Jongh, van Dijk, Hutschemaekers, \& Verbraak, 2017). Here, a patient will complete a questionnaire before each therapy session, from which the therapist can infer whether symptoms deteriorated or improved. Lambert et al. (2005) used a colour scheme: white means that a patient functions normally and that the therapy can be terminated, green means that the patient adequately reacts to the treatment and treatment should be continued, and yellow means that the patient is not making sufficient progress and that the treatment plan has to be adapted. Red indicates that a patient has made no progress and is at risk to experience unwanted events. A meta-analysis by Shimokawa, Lambert and Smart (2010) showed that when therapists used such a feedback system, patient deterioration significantly declined. By means of feedback, a therapist can become aware of how the patient experiences the therapeutic relationship. Making mistakes is sometimes inevitable, but there is always room for improvement. Monitoring and feedback instruments should be incorporated in clinical practice. Because if a therapy does not help, it can have harmful consequences. Therefore, more research (e.g., RCTs) into therapeutic side effects should be conducted so that (1) therapists can be adequately trained about therapeutic side effects; and (2) patients can be completely informed (by means of information leaflets).

The EMDR leaflet includes some information about possible effects after an EMDR session. It states that the EMDR effects might continue after the session has ended and that new emotions might arise. It emphasizes that this is a good thing and patients are advised to write these new emotions and cognitions in a diary. In the last phases of the EMDR protocol (phases 7-8; Appendix 1), an evaluation occurs, but this evaluation is only focused on the most positive aspect a patient has learned about him or herself. No feedback on the intervention and therapist is included in the protocol. De Jong, van Sluis, Nugter, Heiser, and Spinhoven (2012) reported that patients who were not on track improved when therapists were open to receiving and using patient feedback. Furthermore, including the patient's feedback can enable the involvement of the patient (Hovland, Ytrehus, Mellor-Clark, \& Moltu, 2020). Feedback has the potential to contribute to the effectiveness of therapy and might play an important role in preventing therapy failures and its (legal) consequences. 


\section{Chapter 7}

\section{Legal Consequences}

Patients who experienced a traumatic event are likely to be treated with EMDR. After, or during, the therapeutic intervention, a patient can decide to press charges against the alleged perpetrator. As a victim, they have to submit an eyewitness statement to the police. In cases such as sexual abuse, oftentimes no independent evidence is available, and the reliance on the eyewitness statement as main evidence can be problematic. The psychological treatment may have influenced the victim's memory of the event. This is an important aspect for a lawyer and judge to be aware of (Shobe \& Kihlstrom, 2002).

Legal psychologists argue that the link between psychotherapy and legal fact-finding is not a good match. Therapists do no examine the memory of their patients or search for corroborative evidence to support the memory during a therapy session. However, as has become clear from this dissertation, EMDR therapist strongly belief in repressed memories (Chapter 4). Such beliefs can lead to suggestive questioning, which can result in the reporting of misinformation when patients performed eye movements as used in EMDR (Chapter 5). However, even when therapists refrain from such suggestive practices, eye movements itself can induce spontaneous false memories (Chapter 6).

When a patient who has a false memory enters the legal arena, it can have consequences for the court. Specifically, false memories can result in eyewitness errors. For example, eyewitness statements can contain incorrect details or wrongful eyewitness identifications can be made during a line-up. The current dissertation is not the first to indicate possible side effects of EMDR. Leer and colleagues (2017, Experiment 2; 2020) indicated side effects regarding memory accuracy. In both studies, participants underwent a conditioning phase in which two pictures of male faces were followed by a shock. Memory recall was performed with or without making eye movements as used in EMDR. One group completed a discrimination task twice (immediately after and one day later) and another group one day after the intervention. In line with Experiment 1 from Chapter 6, no immediate effects were found. However, in line with Experiment 2 from Chapter 6, when memory was tested after a time delay, false positive rates (i.e., identifying an incorrect picture of a male face) increased. Hence, eye movements can alter memory itself. For legal practice, the decrease in memory accuracy (Leer et al., 2017; 2020) is problematic when a patient has to perform a line-up procedure, because it can lead to a misidentification and eventually result in a wrongful prosecution. To illustrate its importance, data from the Innocence Project (2020) has indicated that around $77 \%$ of wrongful convictions were due to eyewitness misidentifications. 
A false conviction implies two errors: the false conviction itself (i.e., convicting an innocent individual) and a failure to convict the real perpetrator (i.e., the actual perpetrator remains free; Clark, Benjamin, Wixted, Mickes, \& Gronlund, 2015). Eyewitness errors, amongst others, are a prominent cause of wrongful convictions (i.e., 259 out of 337 cases; Innocence Project case files; http://www.innocenceproject.org) and therefore contribute to miscarriages of justice. To counter this, it is of importance that psychologists are aware of the difference between therapeutic treatments and criminal investigations. During therapy, the (emotional) well-being of the patient is the main concern of the therapist. If a patient claims to have encountered a traumatic event, the therapist will take this as a starting point without further examination of its authenticity, because questioning the memory's accuracy could harm the therapeutic relationship. The patient's interests are the main focus, while in criminal investigations the main focus is to uncover the objective truth (Crombag \& Merckelbach, 1996; 2019). In criminal investigations, the interest is to find the perpetrator and impose punishment accordingly. It is therefore problematic to assume that the supposed traumatic event discussed during therapy represents the objective course of events.

The popularity of EMDR is visible in court cases as well. When searching the term "EMDR", a total of 846 cases (June, 2020; www.rechtspraak.nl) emerge, about half of them involve criminal cases. The majority of these cases concern decisions regarding TBS impositions (detention under hospital orders) for the perpetrator. However, a few cases concern the accuracy of an eyewitness who had been treated with EMDR. In one case (ECLI:NL:RBDHA:2018:13856), three women stated to be abused by an acquaintance. The abuse would have occurred years ago and all three women were treated with EMDR to process the traumatic incident prior to the start of the legal proceedings. Two women decided to press charges, the third woman only provided an eyewitness statement. Expert witness work indicated that EMDR therapy elicited false memories and that true memory decreased, thereby threatening the reliability of the statements. Eventually, the court ruled a compensation of $€ 55.000$ was declared inadmissible, because the judge concluded that all three eyewitness reports were unreliable as evidence.

In court cases such as this one, and as discussed in the General Introduction (Chapter 1), allegations involving recovered or false memory claims are referred to the LEBZ in the Netherlands. In Belgium, no such expertise group exists. Though the actual number of false prosecutions and/or convictions in which EMDR has played a role is unknown in the Netherlands as well, it might be the case that this number is even higher in Belgium. Recently, the limitation period for sexual abuse cases in Belgium was abolished (Deferme \& Otgaar, 2020). As a result, 


\section{Chapter 7}

the court will be confronted with sexual abuse cases that might be based on (false) memories of the past. An expertise group such as the LEBZ could help the public prosecution to make substantiated decisions on whether or not to continue the criminal investigation.

\section{Practical Relevance}

An important matter for the implications of this dissertation is to determine to what extent the observed effects in experimental studies are practically relevant (see Table $\mathbf{7 . 2}$ for an overview of effect sizes found in this dissertation). To determine its relevance, the smallest effect size of interest might be informative (Lakens, Scheel, \& Isager, 2018). In the area of legal psychology, not much attention has been given on what might be designated as the smallest effect size of interest. Recently, Otgaar, Manguilli, and Howe (2020) argued that in legal cases, the misremembrance of already one detail (e.g., forgotten or falsely remembered) can have adverse consequences in a legal case. For example, if a patient spontaneously, but falsely, remembers after EMDR therapy that another individual was present during the abuse, such an extra detail might lead the police to question an innocent suspect. When looking at the effect sizes concerning the effects of eye movements on false memory formation (see Table 7.2), it becomes evident that participants in the eye movement conditions on average falsely remembered one detail (e.g., one extra critical lure; see Chapter 6) more than control participants did. For example, in Experiment 2 of Chapter 6 (see Table 7.3): Participants who performed eye movements falsely recalled and recognized one critical lure more than control participants did. Although this difference might be small in size, such a difference might be perilous in legal cases because falsely remembering even one extra detail might lead to negative consequences (e.g., false accusation).

Table 7.2 Overview of Observed Effect Sizes and 95\% Confidence Intervals in Current Dissertation.

\begin{tabular}{llcc}
\hline & Source & Cohen's $\boldsymbol{d}$ & $\mathbf{9 5 \%} \mathbf{C l}$ \\
\hline $\begin{array}{l}\text { EM and suggestion-based false } \\
\text { memories }\end{array}$ & Chapter 5 & 0.77 & {$[0,27,1.16]$} \\
& van Schie \& Leer, 2019 & 0.06 & {$[-0.21,0.34]$} \\
& Calvillo \& Emami, 2019 & 0.02 & {$[-0.34,0.38]$} \\
EM and spontaneous false & False recall (Chapter 6, Exp. 1) & & \\
memories & False recall (Chapter 6, Exp. 2) & -0.24 & {$[-0.72,0.21]$} \\
& False recognition (Chapter 6, Exp.1) & 0.51 & {$[0.03,0.99]$} \\
& False recognition (Chapter 6, Exp. 2) & 0.60 & {$[-0.26,0.67]$} \\
\hline
\end{tabular}

Notes. $\mathrm{EM}=$ Eye Movements. $\mathrm{Cl}=$ Confidence Interval. 
Table 7.3 Means of False Recall and False Recognition in Experiment 2 of Chapter 6.

\begin{tabular}{lcccc}
\hline & EM $(\boldsymbol{n}=\mathbf{3 4})$ & Control $(\boldsymbol{n}=\mathbf{3 4})$ & Cohen's $\boldsymbol{d}$ & $\mathbf{9 5 \%} \mathbf{C l}$ \\
\hline Mean (SD) false recall & $0.27(0.17)$ & $0.19(0.14)$ & 0.51 & {$[0.03,0.99]$} \\
Mean (SD) false recognition & $0.77(0.21)$ & $0.64(0.22)$ & 0.60 & {$[0.12,1.09]$} \\
\hline
\end{tabular}

Notes. $\mathrm{EM}=$ eye movements. $\mathrm{Cl}=$ Confidence Interval.

Patients who are a victim of, for example, sexual abuse deserve the right to press charges against their perpetrator and to receive adequate treatment in case they are unable to cope with the traumatic incident. With this in mind, based on the results described in this dissertation, it is of utmost importance that EMDR therapists (1) are aware of the reconstructive aspect of (traumatic) memory and how this may undermine the quality of legal evidence; (2) refrain from using psychological myths as metaphors to elicit a traumatic memory; (3) document memories that are present before the start of the psychological intervention and "pause" memories that arise during therapy; (4) follow the exact EMDR protocol and prevent therapist drift (Waller, 2009; Waller \& Turner, 2018) and prevent asking additional (suggestive) questions. The court should take note of all documents regarding the therapeutic intervention (e.g., followed procedure).

\section{Conceptual Remarks}

A number of conceptual remarks of the research conducted in this dissertation warrant attention. Though some (methodological) remarks have already been addressed throughout this chapter, I will raise some additional points.

The findings of Chapter 4 showed that EMDR practitioners have strong beliefs in the controversial concept of repression. Though unconscious repression is a commonly used concept to explain recovered memories of abuse and scientific evidence is lacking (Otgaar, Wang, et al., 2019), this does not mean that the scientific community is concordant. Some memory scholars (see e.g., Brewin et al., 2019) argue that instead of unconscious repression, conscious repression (i.e., motivated forgetting or memory suppression) might underlie the phenomenon of recovered memories of abuse. Memory suppression is often assessed by using the Think/No Think task (TNT; e.g., Anderson \& Green, 2001). The TNT task consists of several phases. Participants have to study neutral cue - target word pairs (e.g., tattoo - uncle). in the following phase, participants either have to respond (e.g., when tattoo is presented, participants had to respond with "uncle') or suppress (e.g., participants were instructed to keep the target word (uncle) out of awareness). During a final recall phases, all cues are 


\section{Chapter 7}

presented and participants are instructed to respond to all cues. The general key finding is that suppressed items are recalled worse than baseline (i.e., respond) items. However, replication studies could not find this effect (e.g., Bulevich, Roediger, Balota, \& Butler, 2006). Wessel, Albers, Zandstra, and Heininga (2020) performed a multiverse analysis on several memory suppression experiments. They could not find consistent evidence for suppression effects. Although unconscious repression was, explicitly, examined in Chapter 4, researchers (66\%; Chapter 4, Study 2) and students (around 88\%; Chapter 4, Study 1 and 2) strongly believe in repression. This might make one wonder how the scientific literature and educational programs contribute to these controversial beliefs.

Another important remark is that the studies primarily relied on (healthy, psychology) student samples. Therefore, the experimental results of Chapters 2, 5, and 6 cannot directly be generalized to a clinical population. A next logical step would be to examine false memory susceptibility due to EMDR in clinical populations. When doing this, it might be the case that for EMDR to be able to alter the autobiographical memory (i.e., making it less vivid and emotional), therapists have to take the risk that there is always a likelihood for false memories to emerge. In other words, it might not be possible for EMDR therapists to degrade the memory vividness and emotionality without eliminating the risk of false memory formation. As described in this General Discussion, this situation (i.e., when a new memory surfaces) should be approached cautiously.

A final remark is that although it has been shown that EMDR can lead to false memories (Chapters 5 and 6), these results were obtained in a controlled manner. However, in clinical practice, the exact causal relationship between EMDR and false memories remains unknown. A patient can recover a traumatic memory during a period in which she receives psychological treatment, but that does not mean that the treatment is the cause of this recovery (e.g., there might be an external cue leading to the recovered memory).

\section{Future Research}

As research on therapeutic side effects is highly understudied, many questions await further investigation. By broadening our knowledge about therapeutic side effects and its effect on legal proceedings, therapeutic interventions could be improved to prevent future missteps.

As the Dutch EMDR protocol is reviewed each year, based on therapists' experiences and scientific results, it would be an opportunity to adapt the instructions to identify the target image. Clinicians and researchers could collaborate on an instruction that, on the one 
hand, elicits details useful for the desensitization phase, but on the other hand is in line with correct information about how memory operates. This new instruction could be piloted in both laboratory and clinical research to examine whether it influences the therapeutic relationship and benefits accurate memory.

EMDR was initially developed to treat PTSD. Before EMDR was scientifically supported, the eye movement treatment was adopted around the world. However, some patients did not prefer performing eye movements, so other alternative tasks were used in clinical practice, again without being substantiated by scientific results. To validate the use of alternative dual tasks, more experimental studies and/or randomized clinical trials should be conducted. These studies should be of high quality (e.g., methodologically) and have a low risk bias. Next, meta-analyses including enough high quality studies could determine its overall effectiveness and conclude whether or not the use of alternative dual tasks is justified. Due to the rapid therapeutic effects that are ascribed to EMDR (i.e., PTSD patients only need a few EMDR sessions), disorders other than PTSD are treated with EMDR as well. However, as mentioned before, the use of EMDR to treat other disorders is not advisable. A modest proposal would be to make sure that EMDR is completely effective, especially on the longterm, for the disorder it aimed to treat in the first place (i.e., PTSD) before applying it to other disorders.

In general, it is important for therapists to stay up-to-date with scientific knowledge about how memory operates. Because therapists work with autobiographical memories, they have to know, for example, what type of information (e.g., central or peripheral details) an individual might remember after a traumatic incident, what questions can be asked to prompt correct information (e.g., open questions) and what questions or prompts should be avoided because they may wrongfully influence memory. Currently, therapists can take several trainings to acquire or refresh their knowledge about certain therapeutic interventions, but these are not mandatory. It would be interesting to include a mandatory training in the $\mathrm{RINO}^{9}$ educational programs on how (traumatic) memory (does not) functions (including knowledge on the concept of repression) and how to apply this in practice. This training would not only be relevant to EMDR practitioners, but to all therapists. This proposed training could be repeated after a certain time span. Though it might be difficult to counter such strong beliefs, it could be substantiated whether the training was effective (i.e., the therapist remains to have correct knowledge and applies it correctly in practice) by retesting. This exact same procedure could be used to make therapists aware of therapeutic side effects. Therapists should be able to

${ }^{9} \mathrm{RINO}$ is a nationwide operating organization for continuing education and 


\section{Chapter 7}

recognize therapeutic side effects and be able to reason about their probable cause by means of monitoring and feedback systems. By doing this, the scientist-practitioner gap could be identified and reduced.

Inherent to experimental studies is the use of materials that are approved by an ethical committee. To do so, the materials used in the current dissertation are of negative valence, but not equivalent to traumatic experiences. To test the susceptibility to suggestive false memories, future studies could include misinformation presented by an individual with authority (e.g., the experimenter). In this way, the misinformation will be more explicitly mentioned to the participant, as what would happen when a therapist (unintentionally) provides such misinformation. In addition, because the EMDR kit is used in clinical practice, it would be advisable to use this kit in experimental studies as well. Because the ground truth is not known when targeting autobiographical memories, virtual reality techniques, for example, are advised to use in future studies to tap into autobiographical memories.

Therapeutic side effects entail more than the production of suggestion and spontaneous false memories. Future studies should also look into possible other side effects, such as: having trouble sleeping, having nightmares, worsening of symptoms on the longer term, and feelings of shame.

When comparing successful and unsuccessful therapies, the therapeutic relationship seems to play an important role (Hafkenscheid, 2014). Several studies by Werbart and colleagues $(2015$; 2018; 2019) showed that the mutual liking between a therapist and patient is predictive of the treatment outcome. Patients who deteriorated during therapy claimed that the therapeutic relationship was distant and artificial. Werbart and colleagues (2015) reasoned that in these cases, the therapists ignored the needs of the patients and, consequently, they could not benefit from the therapy. Looking at unsuccessful treatments more closely, it becomes evident that both therapist and patient evaluated the therapeutic relationship to be distant at the beginning of therapy. Based on this reserved relationship, the therapists were not capable to adapt the treatment plan accordingly (Werbart et al., 2018). For successful treatments, both therapist and patient felt a strong connection: they felt sympathetic, thoughtful and liked each other (Werbart, Missios, et al., 2019). Though the previous studies of Werbart and colleagues concerned different therapists, the role of therapeutic alliance is also evident for therapists who have had successful and unsuccessful treatment outcomes. When asked to elaborate on why a treatment outcome was unsuccessful or successful, therapists reported that the patient did not want to work on the problems or 
that they felt connected and had a common goal, respectively (Werbart, Annevall, \& Hillblom, 2019). Because the therapeutic relationship is a strong indicator of therapy outcome, it would be interesting to see whether an initial matching program (e.g., Tinder for psychotherapists; Houben, 2020) would be able to optimize the therapeutic relationship.

Currently, the patient's progress can be evaluated during routine outcome monitoring (ROM) by means of several questionnaires, such as the Outcome Rating Scale (ORS; Miller, Duncan, Brown, Spark, \& Claud, 2003), Session Rating Scale (SRS; Duncan et al., 2003), and Outcome Questionnaire-45 (OQ-45; Lambert et al., 2004). However, if a therapist does not understand the patient, there will be a mismatch between what a patient needs and what the therapist offers (Gold \& Striker, 2011). Monitoring the therapeutic relationship is a continuous responsibility of the therapist, but these ROM questionnaires do not consider the therapist perspective and, hence, it is difficult for a therapist to adopt a "meta-perspective". Therapists can complete the Therapist Satisfaction Scale (TSS; Kokotovic \& Tracey, 1990) and the Session Evaluation Questionnaire (SEQ; Stiles, 1980) after each therapy session. Scientific studies on the TSS and SEQ are scarce and it is unknown whether these questionnaires actually contribute to the therapeutic relationship and therapy outcome. Although some aspects of the therapeutic relationship are more difficult to measure or even not possible to measure (e.g., projection of feelings), such questionnaires might uncover blind spots. A major shortcoming of the ORS, SRS, OQ-45, TSS, and SEQ is that they do not specifically ask about unwanted events. Even when the therapeutic relationship is good, side effects can occur. More research into (new) therapy progress questionnaires is necessary.

In a world with seven billion inhabitants, mental health problems will always be present. It is up to therapists to provide the best quality of care to help these individuals living their daily life. This best quality of care includes a therapist having adequate knowledge, providing a patient with complete information (i.e., not only the advantages but also possible side effects) about the therapeutic intervention, and refraining from suggestive questions. Keeping the legal consequences in mind, awareness of the memory consequences of therapeutic interventions should be highly encouraged.

\section{Conclusion}

The main aim of the current dissertation was to examine the legal psychological effects of EMDR. Chapters 2 and 3 highlighted some possible pitfalls in the EMDR protocol itself. Next to that, Chapter 4 indicated that EMDR therapists are not sufficiently knowledgeable 


\section{Chapter 7}

about the functioning of memory when it comes to issues such as repressed memories. Furthermore, Chapters 5 and 6 presented that participants are susceptible to both suggestive and spontaneous false memories after performing eye movements. These results combined with research from others labs (e.g., Leer \& Engelhard, 2020) demonstrate that from a legal psychological perspective, EMDR can be quite risky when it appears in court cases. Specifically, others and I have shown that (i) EMDR therapists strongly believe in repressed memories, (ii) memory myths are used in Dutch EMDR protocols, and (iii) eye movements can lead to false memories. All these findings can lead to serious ramifications in legal cases such as false accusations or suggestive therapeutic interviewing techniques.

Although EMDR is considered to be an evidence based treatment and is highly popular worldwide, this does not mean that it is justified to ignore possible side effects or to use certain instructions without scientific validation. Even when a patient does not press charges, side effects (e.g., a false memory) could still have an immense impact on one's personal life (e.g., no contact with family members; Patihis \& Pendergrast, 2019). For both clinical and legal practices, more research on the memory effects of EMDR is needed to draw firm conclusion about the use of the current protocol.

To prevent an amplification of the scientist-practitioner gap, more channels to communicate scientific findings should be found to present relevant information and to prevent misinterpretation of scientific results. This exchange is, just as the therapeutic relationship, is a two-way-street: both researchers and clinicians should be open to discuss and work together to improve current mental health practices. 



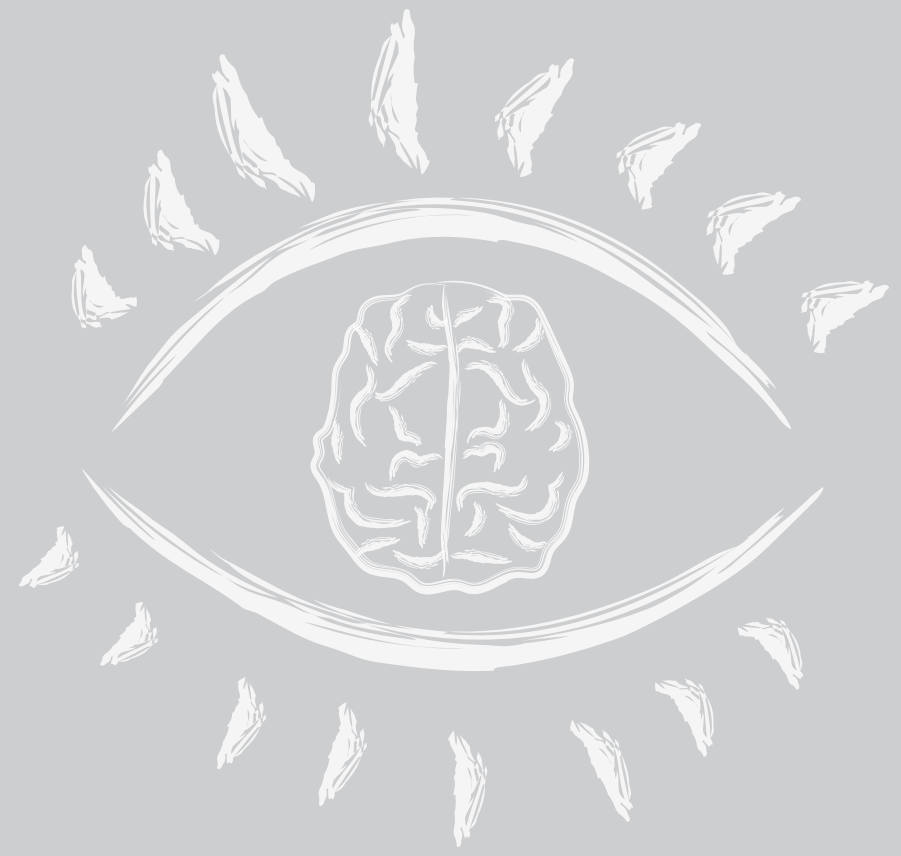


Summary

Samenvatting 



\section{SUMMARY}

Eye Movement Desensitization and Reprocessing (EMDR) is a popular and effective psychological intervention to treat patients who have experienced a traumatic event. The main aim of this dissertation was to examine the legal psychological context of EMDR by examining (i) EMDR therapists' knowledge about the functioning of memory and (ii) its potential to induce false memories (i.e., memories of details and/or an event that an individual did not experience).

In Section I (Chapters 2 and 3), general aspects of the EMDR procedure are discussed. In the Dutch EMDR protocol, EMDR practitioners are able to choose between two instructions to identify the target image. These instructions are, however, psychological myths. Chapter 2 described individuals' expectations after receiving one of the two metaphoric instructions (i.e., video or photobook instruction) as used in the Dutch EMDR protocol. The results of this study showed that especially participants who received the video metaphor expected to recall a memory more emotional and vivid as compared with the photobook metaphor and control instruction. It is beneficial for the desensitization phase in EMDR for a memory to be highly vivid. However, this expectancy effect may lead patients to report as many details as possible, irrespective of whether they are correct. Chapter $\mathbf{3}$ meta-analyzed the effect of eye movements and alternative dual tasks on vividness and emotionality in laboratory studies. Though EMDR emphasizes the importance of eye movements, alternative dual tasks (e.g., counting) are used to obtain the same beneficial effect (i.e., reduced vividness and emotionality). Eye movements and alternative duals had similar effects on vividness and emotionality ratings, although the effect was stronger for vividness ratings. This finding suggests that vividness might play a larger role than initially assumed.

Section II (Chapters 4, 5, and 6) focused on EMDR therapists' knowledge about the functioning of memory and on the link between eye movements, as used in EMDR, and false memories. Chapter $\mathbf{4}$ presented the results of a survey of EMDR practitioners about their beliefs about the functioning of memory, including the belief in repressed memories. Because EMDR practitioners work with autobiographical memories during a therapy setting, it is imperative that they are knowledgeable enough about how memory operates. Although EMDR practitioners had sufficient knowledge on several aspects concerning memory functioning, they still strongly believe in the controversial concept of unconscious repression. This can be problematic when a therapist seeks for repressed memories, thereby increasing the likelihood of false memory formation. Chapter 5 presented an experimental study testing the susceptibility to suggestion- 


\section{Summary}

induced false memories after performing eye movements, as used in EMDR. EMDR therapists could, unintentionally, provide misinformation to their patients (e.g., suggesting event X might have happened to them and therefore they experience these symptoms). Results showed that participants who performed eye movements answered questions correctly less often and endorsed more misinformation than participants who did not perform eye movements. A therapy could still induce false memories when no suggestive information is presented. Chapter 6 therefore examined the spontaneous false memory effect of eye movements as used in EMDR in two experiments (immediate and delayed memory test). When memory was tested 48 hours later, results indicated that participants who performed eye movements showed higher true and false memory ratings.

In Chapter 7, the most important findings of the above mentioned chapters and their practical implications were discussed. To conclude, this dissertation has shown that eye movements as used in EMDR might have adverse effects on memory. 


\section{SAMENVATTING}

Eye Movement Desensitization and Reprocessing (EMDR) is een populaire en effectieve psychologische interventie om patiënten die een traumatische ervaring hebben meegemaakt te behandelen. Het belangrijkste doel van dit proefschrift was om de rechtspsychologische context van EMDR te onderzoeken door (i) de kennis van EMDR therapeuten over de werking van het geheugen te onderzoeken en (ii) de vatbaarheid voor pseudoherinneringen (d.w.z. herinneringen aan details en/of gebeurtenissen welke een individu niet heeft meegemaakt) te onderzoeken.

In Sectie I (Hoofdstukken 2 en 3), worden algemene aspecten van de EMDR procedure besproken. In het Nederlandse EMDR-protocol kunnen EMDR-therapeuten kiezen uit twee instructies om het naarste aspect van de traumatische ervaring te identificeren. Deze twee instructies staan echter bekend als psychologische mythen. Hoofdstuk 2 beschreef de verwachtingen van proefpersonen nadat ze één van de twee metaforische instructies (d.w.z. video of fotoboek instructie) hadden ontvangen, zoals gebruikt in het Nederlandse EMDRprotocol. De resultaten van deze studie laten zien dat voornamelijk proefpersonen die de video instructie ontvingen, in vergelijking met de fotoboek en controle instructie, verwachtte om een levendige en emotionele herinnering op te halen. Voor de desensitisatie fase in EMDR kan het voordelig zijn als de herinnering zo levendig mogelijk is. Echter, dit verwachtingseffect kan ertoe leiden dat een patiënt denkt zo veel mogelijk details te moeten rapporteren, ongeacht of deze waargebeurd zijn. Door middel van een meta-analyse werd in Hoofdstuk $\mathbf{3}$ het effect van oogbewegingen en alternatieve duale taken op levendigheid en emotionaliteit onderzocht in laboratoriumonderzoeken. Alhoewel EMDR de waarde van oogbewegingen benadrukt, worden er in de praktijk ook andere duale taken (bv. tellen) gebruikt om hetzelfde effect te behalen (d.w.z. een reductie in levendigheid en emotionaliteit). Oogbewegingen en alternatieve duale taken hebben gelijke effecten op levendigheid en emotionaliteit, al was dit effect sterker voor levendigheidscores. Deze bevinding suggereert dat levendigheid een belangrijkere rol speelt dan aanvankelijk werd aangenomen.

Sectie II (Hoofdstukken 4, 5, en 6) richtte zich op de kennis van EMDR therapeuten over de werking van het geheugen en de relatie tussen oogbewegingen, zoals gebruikelijk in EMDR, en pseudoherinneringen. Hoofdstuk 4 presenteerde resultaten van een enquête waarin EMDR-therapeuten werd gevraagd naar hun kennis over de werking van het geheugen, inclusief kennis over hervonden herinneringen. Omdat EMDR-therapeuten werken met autobiografische 


\section{Samenvatting}

herinneringen tijdens een therapiesessie, is het belangrijk dat ze beschikken over voldoende kennis over de werking van het geheugen. Alhoewel EMDR-therapeuten voldoende kennis hadden over bepaalde aspecten van hoe het geheugen werkt, geloven ze ook nog sterk in het controversiële concept onbewust verdringing. Dit kan problematisch zijn, omdat een therapeut tijdens de sessie op zoek kan gaan naar zulke herinneringen, waardoor de kans op een pseudoherinnering vergroot. Hoofdstuk 5 presenteerde een experimentele studie om de vatbaarheid voor suggestieve pseudoherinneringen na het maken van oogbewegingen, zoals gebruikelijk in EMDR, te onderzoeken. EMDR-therapeuten kunnen, onbedoeld, misinformatie presenteren aan hun patiënt tijdens een sessie (bv. ze kunnen suggereren dat gebeurtenis $X$ heeft plaatsgevonden en dat de patiënt daardoor symptomen ervaart). De resultaten laten zien dat proefpersonen die oogbewegingen hebben gemaakt minder vaak vragen correct beantwoorden en vaker misinformatie rapporteerden dan proefpersonen die geen oogbewegingen maakten. Een therapie kan ook pseudoherinneringen ontlokken als er geen suggestieve informatie wordt aangeboden. Hoofdstuk 6 onderzocht in twee experimenten (onmiddellijke en latere geheugentest) de vatbaarheid voor spontane pseudoherinneringen na het maken van oogbewegingen. Wanneer het geheugen 48 uur later werd getest, laten de resultaten zien dat proefpersonen die oogbewegingen maakten meer correcte, maar ook meer pseudoherinneringen hadden.

In Hoofdstuk 7 werden de belangrijkste bevindingen van de voorgenoemde hoofdstukken besproken, alsmede de praktische implicaties. Op grond van bovengenoemde resultaten kan worden geconcludeerd dat oogbewegingen zoals gebruikelijk in EMDR nadelige effect kan hebben voor het geheugen. 



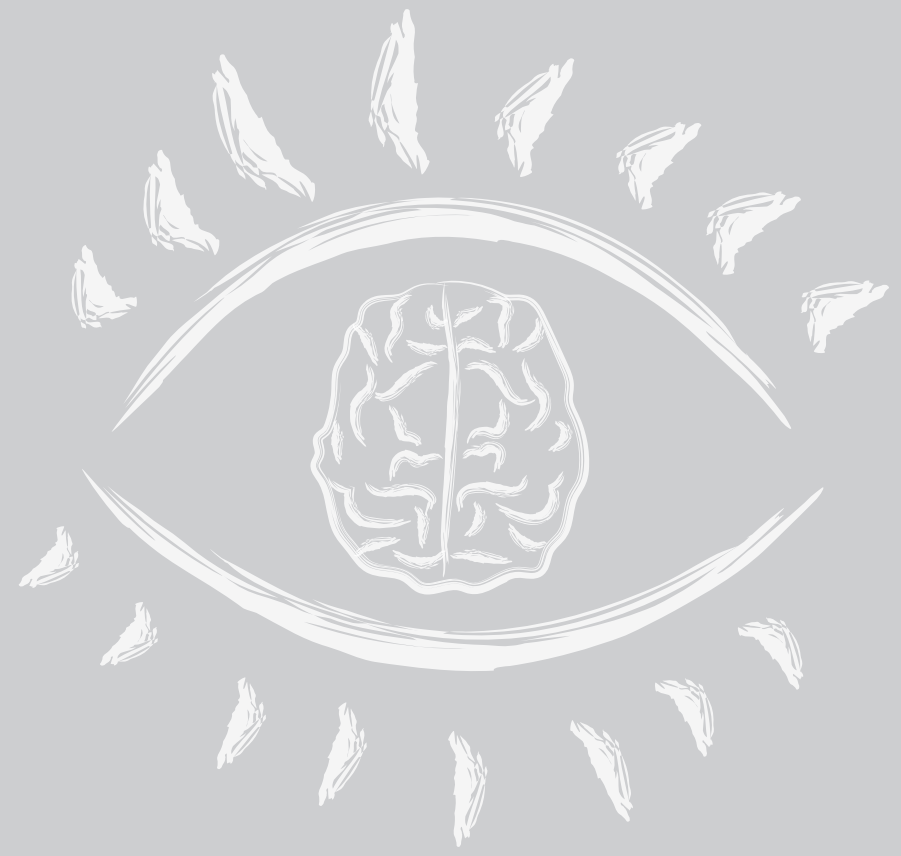


Impact 

This impact chapter addresses the scientific and clinical implications of the results discussed in this dissertation. Besides its theoretical relevance, the research presented in this dissertation can have ramifications for society. Though many of these practical applications have been discussed in the previous chapters, several key aspects will be highlighted in this chapter. More specifically, the following aspects will be highlighted: (i) research (i.e., what is the main aim of the dissertation and its conclusions); (ii) relevance (i.e., what does the dissertation contribute to science and society); (iii) target group (i.e., for whom are the findings reported in the dissertation relevant and why); and (iv) activity (i.e., how to involve the target group so that the acquired knowledge can be used).

Research. The main aim of this dissertation was to examine the (legal) psychological effects of Eye Movement Desensitization and Reprocessing (EMDR). EMDR uses well-known psychological myths (e.g., memory works as a video camera) to identify the target image. Such instructions, however, can lead to expectancy effects, which may motivate patients to report more (false) details as they originally remembered. In addition to this, the instructions and a therapist providing such an instruction were not evaluated positively. That is, the instructions were sometimes evaluated as vague and the therapist providing the instruction as unreliable. A therapeutic relationship is important for a successful treatment outcome and these views may jeopardize this. Moreover, performing eye movements during memory retrieval is a key component of EMDR and its aim is to diminish the emotional impact of traumatic memories (Shapiro, 2002). However, alternative dual tasks obtained a similar effect. Furthermore, vividness seemed to be affected more strongly by eye movements (or alternative dual tasks) than emotionality. Hence, the role of vividness might be more prominent than initially assumed.

As EMDR therapists work with autobiographical memories it is imperative that they are aware of how memory functions. Although EMDR therapists (and students and researchers as well) have some sufficient knowledge about the functioning of memory (i.e., they are aware that (traumatic) memory is reconstructive), they hold strong beliefs in the controversial concept of repressed memories. This is highly problematic as it can lead to actively seeking repressed memories, thereby increasing the likelihood of false memory formation.

Concerning the memory effects of eye movements as used in EMDR, this dissertation focused on the susceptibility to suggestion-induced false memories and spontaneous false memories. By using well-known false memory paradigms (e.g., misinformation paradigm and DRM paradigm), the results of this dissertation suggest that after performing eye movements, 
Impact

individuals might become more susceptible to both suggestion-induced and spontaneous false memories. Hence, eye movements as used in EMDR might lead to therapeutic side effects. More specifically, when misinformation is suggested after performing eye movements, a patient might endorse this into one's own memory of the event. However, even when the therapy is adequately performed by a therapist, performing eye movements can still lead to the formation of spontaneous false memories because it increases the associative link between memories.

Relevance. The relevance of the findings reported in this dissertation lies in its implications. Individuals who encountered a traumatic experience and who are not able to cope adequately with this are at risk to develop PTSD. In such cases, EMDR will be a first choice of treatment. However, unlike medicine, potential side effects of therapeutic interventions are unknown. Such knowledge is important for all therapeutic interventions, but especially for a therapy that gained immense popularity without a solid theoretical background. The results discussed in this dissertation and reported by others (e.g., Leer et al., 2017; 2020) indicate that eye movements lead to memory impairing effects. Hence, when an individual has been treated with EMDR before filing a report to the police, the reliability of the eyewitness statements is questionable.

While research in the area of therapeutic side effects is still in its infancy, the findings from this dissertation serve as a foundation toward a more comprehension of these concerns on the short-term and to serve as an encouragement for future research to examine potential therapeutic side effects.

Target group. The results of this dissertation are targeted at both scientific and clinical professionals. The subject of psychological therapeutic side effects is highly understudied in the academic field (Rozental et al., 2016). Knowledge about possible side effects has to be established in a laboratory setting before applying research in the clinical field. Therefore, it is up to researchers to examine potential side effects of psychological interventions by means of high quality studies.

The other target group for this dissertation, clinicians, are the professions who apply scientific knowledge in the field. Based on scientific results on the functioning of memory and therapeutic side effects, they have to know (i) what possible side-effects could occur; (ii) how to prevent them; (iii) how to recognize them and (iv) how to solve them. Though the prevention and recognition of side effects might seem more important, it is only logical that therapists have to know what they have to look out for. This dissertation addressed the 
first point and only one kind of therapeutic side effect. For clinicians to effectively apply this knowledge in practice, researchers may want to catch up on the importance of examining therapeutic side effects. Lastly, the results of this dissertation could be of interest to legal practitioners (e.g., judges, lawyers). These professions are concerned with evaluating the credibility of eyewitness testimonies. For example, objective evidence is oftentimes absent in sexual abuse cases and eyewitness testimonies are the only evidence to rely on. Legal professionals have to be aware of the potential problems EMDR entails, so they benefit from knowledge regarding therapeutic side effects, because victims of traumatic events are most likely to be treated with EMDR.

The findings reported in this dissertation require additional research and replication before robust recommendations can be offered to practitioners working in these contexts. However, this does not mean that all three practitioner groups (i.e., researchers, clinical and legal practitioners) should discard potential pitfalls of therapeutic interventions.

Activity. All target groups can be informed about the findings discussed in this dissertation in multiple ways. Researchers can be reached in the usual manner by means of scientific conferences and scientific publications. Scientific publications should be open access so that clinicians can access these as well. A way to promote research on the topic of therapeutic side effects, researchers could work with open data (e.g., the Open Science Framework). In this way, materials, procedure and data will be available and this might increase the quality of scientific studies.

Clinicians can be reached by several options. First, knowledge on therapeutic side effects (e.g., false memories) and knowledge about the functioning of memory should be implemented in their mandatory education. Moreover, trainings about these topics should be followed more than once to keep one's expertise level high. There are clinical conferences were clinicians could be informed about scientific findings as well. However, to ensure this, researchers examining this topic should also be accepted to present their findings on clinical conferences. At such conferences, they can inform clinicians directly and in a correct manner, before misinterpretations might circulate in certain therapeutic groups. Moreover, clinical practices could also invite researchers to present their findings in their own group. By doing this, there might be more room for questioning than usually is on conferences. Furthermore, magazines of certain therapeutic associations should also be accessible to researchers, because it may give them a look into how matters evolve in clinical practice.

Legal practitioners can be addressed in the same manner as the ones discussed 
Impact

above. But, more specifically, interdisciplinary conferences and journals would benefit this spreading of knowledge.

Finally, all target groups can be informed by means of teaching. This could start as early in Bachelor's and Master's programs as well as in postgraduate studies. For example, specific core courses could implement this or even a specific course could be added to the curriculum.

To diminish the scientist-practitioner gap, the expertise of all professionals must be bridged. Knowledge is achieved by cooperation between all parties involved (i.e., researchers and practitioners) and this should be facilitated, not obstructed. Only then, it might be possible to solve the problematic role of EMDR within legal cases. 



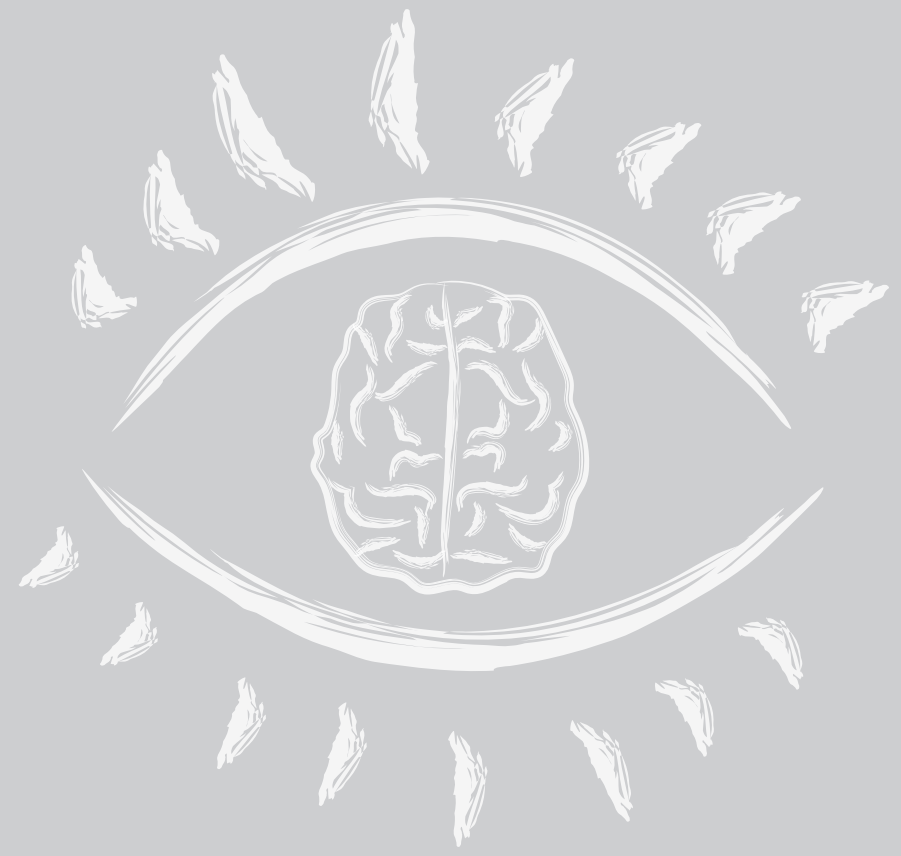


Appendices 



\section{APPENDIX 1 - DUTCH EMDR STANDARD PROTOCOL}

Erik ten Broeke, Ad de Jongh, \& Hellen Hornsveld, versie 2020

\section{Introductie}

Als EMDR wordt ingezet zijn de klachten bekend, is de te bewerken herinnering vastgesteld, en zijn eventuele vermijdingsgedragingen en situaties die vermeden en/of gevreesd worden voor de toekomst (t.b.v. het vaststellen van mogelijke flashforwards en future templates) in kaart gebracht.

\footnotetext{
"Ik ga straks een aantal vragen stellen over jouw herinneringen aan de gebeurtenis waaraan we gaan werken. Op een gegeven moment zal ik je vragen je te concentreren op bepaalde aspecten van de herinnering. Met name zal ik je vragen naar het beeld in je hoofd dat nu nog het naarste is om naar te kijken. Daarna zal ik je vagen om met je ogen mijn vingers te volgen."
}

Introduceer de oogbewegingen. Let op de positie van de stoelen, de achtergrond, de snelheid en de afstand. Indien een andere afleidende taak wordt gebruikt, zoals de lichtbalk, geef dan een demonstratie, bijvoorbeeld van het gebruik van de koptelefoon of van de 'hand-taps'. Alles moet vooraf klaar liggen en zijn gedemonstreerd.

"Ik wil je vragen vanaf het moment dat je mijn hand volgt je min of meer op te stellen als een toeschouwer, die waarneemt wat er door hem/haar heen gaat. Het kunnen gedachten, gevoelens, beelden, emoties of lichamelijke reacties zijn. Alles is goed. Volg maar gewoon wat er opkomt, zonder te sturen en zonder jezelf af te vragen of het wel goed gaat. Richt je aandacht in het begin zoveel mogelijk op de herinneringen, terwijl je mijn hand volgt, die ik zo snel zal bewegen dat je je erg moet inspannen deze goed te volgen, waardoor het erg moeilijk wordt om op de herinnering geconcentreerd te blijven. Dat geeft heelmaal niet; dat is juist te bedoeling. Daarna zal ik je vragen wat er bij je opkomt. Stel je dan open voor wat er spontaan in je opkomt. Ik zal je af en toe vragen opnieuw naar het herinneringsbeeld te kijken om te controleren hoeveel spanning je daarbij nog voelt. Het is onmogelijk om het fout te doen. Richt je aandacht telkens op wat er opkomt....ik zal je door het proces heen leiden". 


\section{Appendices}

\section{2. 'Scherpstellen' (Assessment)}

"Het is de bedoeling dat je nu je ogen sluit en ze pas opendoet als ik je dat vraag...is dat oké?" (niet bij zeer angstige of dissociatieve patiënten of patiënten die het sluiten van ogen weigeren).

\subsection{Traumatische herinnering}

a. Visuele representative van de negatieve herinnering.

"Vertel me dan nu in grote lijnen de nare herinnering, vanaf het punt waar die voor jouw gevoel begint tot het punt waar die voor jouw gevoel echt eindigt. Beschrijf de gehele herinnering in grote lijnen. Het gaat om de herinnering en niet zo zeer om wat er precies is gebeurd."

Laat de patiënt de hele herinnering vertellen en stimuleer hem/haar door, indien nodig, op verschillende moment op een nieuwsgierige toon te vragen: "en hoe gaat de herinnering verder...?" tot het daadwerkelijke einde van de herinnering is bereikt.

Check: "Zijn er - op dezelfde dag - daarvoor of erna nog dingen gebeurd die er voor jouw gevoel bij horen?"

b. Target selectie:

Maak een keuze tussen de filmmetafoor of de fotoboekmetafoor (beide zijn goed).

\section{Film metafoor:}

"Je hebt me net verteld hoe deze herinnering in je hoofd ligt opgeslagen. Nu vraag ik je: wat is op dit moment, als je vanuit het hier en nu naar kijkt, het naarste plaatje van deze herinnering? Kijk als het ware naar de film en zet deze - op de seconde - stil zodat het een plaatje wordt. We zoeken vooral naar een plaatje waarin jij zelf te zien bent. Het gaat er dus niet om wat je destijds het naarste vond, maar wat je nu, op dit moment, het naarste plaatje vindt om naar te kijken, inclusief eventuele plaatjes die pas later in de film zijn terechtgekomen. Hoe ziet het plaatje er uit dat nu, op dit moment, de meeste spanning oproept?"

Of: 


\section{Fotoboek metafoor:}

"Stel dat er een fotoboek bestaat in je hoofd, waarin foto's staan warop te zien is hoe jij je deze gebeurtenismoment nog herinnert... het zijn vooral foto's waarop jijzelf te zien bent....het kan zijn dat er foto's in dat fotoboek staan waarop dingen zijn te zien die niet feitelijk zijn gebeurd, maar pas later in het fotoboek zijn terechtgekomen. Welke foto roept dan nu, op dit moment, de meeste spanning op?....Wat zie jij op die foto?"

Indien er meerdere nare beelden zijn, help de patiënt dan te kiezen:

"Neem beide plaatjes in gedachten, hang ze als het ware, bijvoorbeeld aan een waslijn, naast elkaar op in je hoofd... welke van die twee raakt je dan nu, op dit moment het meest?"

Check: "Even voor de zekerheid, is dit het beeld in je hoofd dat je echt nu, op dit moment, het naarste vindt om naar te kijken?"

Algemeen aandachtpunt: vergewis je er goed van dat dadawerkelijk is gekozen voor een plaatje dat nu, op dit moment de meeste spanning oproept. Dus niet om een moment dat destijds het naarste was.

Eventueel een neutrale werktitel benoemen.

Vraag je af - luisterend naar het verhaal en kijkend naar het gekozen target - in welk domein (vermoedelijk) de negatieve cognitie is te vinden, d.w.z.:

- Controle (bijv. "Ik ben machteloos/hulpeloos")

- Veiligheid (betreft de situatie; bijv. "Ik ben (gevoelsmatig, nog steeds) in gevaar")

- Zelfwaardering (bijv. "Ik ben waadeloos, een stommeling, een slecht mens, een zwakkeling, een lafaard")

- Verantwoordelijkheid/schuld (bijv. "Ik ben schuldig")

\subsection{Negatieve cognitie (NC)}

"Wat we nu moeten uitzoeken is hoe het komt dat juist dit plaatje in je hoofd nu, op dit moment, nog zo naar voor je is als je er naar kijkt, dus los van hoe het destijds voor 


\section{Appendices}

je was. Wat maatk dat dit plaatje nu nog zo naar voor je is?"

Luister goed naar welke thema's je hoort en vraag door totdat duidelijk is of het om het domein 'controle' (zie A), 'veiligheid' (zie B) gaat of om een van de domeinen 'zelfwaardering' of 'schuld' (zie C).

\section{A. Controle}

Indien het antwoord doet vermoeden dat het om controle gaat: 1) de machteloosheid van toen wordt opnieuw gevoeld, of 2) het beeld is erg aversief en naar om te zien.

"Klopt het dat je je (opnieuw) machteloos voelt of dat je er niet tegen kunt als je nu naar het plaatje kijkt?"

Indien dit klopt, ga door met 2.5 (de PC ligt immers al vast), het vaststellen van de emotie.

\section{B. Veiligheid}

Indien het antwoord doet vermoeden dat het om actueel verlies van gevoel van veiligheid gaat (het lijf van de patiënt reageert bij deze herinnering alsof het gevaar er nog steeds is):

"Klopt het dat je je opnieuw in gevaar voelt als je nu naar het plaatje kijkt?" Indien dit klopt, ga door met 2.3, het vaststellen van de gewenste positieve cognitie.

\section{Zelfwaardering en schuld}

Indien je vermoedt dat het beeld een negatieve opvatting over patiënt zelf activeert, herhaal je je vraag:

"Wat maakt dat dit plaatje nu nog zo naar voor je is?"

Indien het antwoord niet direct betrekking heeft op een disfunctionele evaluatie (maar bijvoorbeeld op gedrag: 'dat ik er bij stond en niks deed') helpen de volgende vragen:

"Wat zegt dat over jou als persoon (bijv. dat je niks deed)?/Wie of wat ben je dan?"

"Hoe wordt zo iemand vaak genoemd/Hoe noem je zo iemand?"

Indien het onduidelijk blijft kan de volgende vraag helpen:

"Als je kijkt naar dit beeld, welke uitspraak over jezelf raakt je dan nu, op dit moment, het meest?"

Check: "Even voor de zekerheid: dus als je kijkt naar dat beeld en je zegt in gedachten: 'ik 
ben..., dan raakt dat je nu vooral?"

Indien er meerdere mogelijke NCs zijn waaruit je patient moeilijk kan kiezen:

"Kijk naar dat beeld en zeg in gedachten 'Ik ben.. (NC1)'... blijf naar het beeld kijken en zeg nu 'Ik ben...(NC2)'... welk van de twee uitspraken raakt je op dit moment dan het meest bij het beeld?

NC:

\subsection{Gewenste (positieve) cognitie (PC)}

"Als je dat beeld weer voor de geest halt, wat zou je willen geloven over jezelf, in plaats $\operatorname{van}[N C]$ ?"

- Veiligheid (betreft de situatie): "Ik ben (nu) veilig"

- Zelfwaardering: "Ik ben oké, de moeite waard, competent, sterk" of bijv. "Ik deug"

- Verantwoordelijkheid/Schuld: "Ik heb gedaan wat ik kon" of "Ik ben onschuldig"

- Control: de PC staat vast: "Ik kan het [beeld] aan"

Behulpzame vragen:

"Hoe noem je zo iemand die...?"

"Ken je iemand die geen ...[NC]... is?"

"Hoe noem je diegene die. ...?"

PC:

\subsection{Geloofwaardigheid (VoC 1-7)}

"Terwijl je kijkt naar het beeld in je hoofd, hoe geloofwaardig voelt de uitspraak ... [PC]... dan nu aan op een 7-puntsschaal, waarbij 1 betekent 'volledig ongeloofwaardig' en 7 'volledig geloofwaardig'?'

$\mathrm{VoC}=$

\section{$2.5 \quad$ Emotie}

"Als je het beeld weer in gedachten neemt en tegelijkertijd in gedachten zegt ... [NC]..., welke emotie voel je dan nu, op dit moment?" (Noem eventueel bij wijze van hulp: 
Appendices

"bijvoorbeeld bang, boos, bedroefd")

\subsection{Lading van het beeld (SUD 0-10)}

"Neem het beeld in gedachten en zeg in jezelf ...[NC]..., hoeveel spanning voel je dan nu, op dit moment geschat op een schaal lopen van o, helemaal geen spanning, tot en met 10, maximale spanning?"

\subsection{Plaats van de spanning}

"Waar in je lichaam voel je die spanning het sterkst?"

\section{Desensitizatie}

Hand klaar voor de ogen:

"Kijk naar de toppen van mijn vingers"

a. Neem het beeld in gedachten, zeg in gedachten ... [NC]... Wees je beust van de spanning in je ...[locatie van de spanning]"

Geef even de tijd zich te concentreren.

"Volg mijn hand en stel je open voor wat er daarna spontaan bij je opkomt"

$\ll<<<$ set met afleidende stimulus $\gg>>>$ (circa halve minuut).

b. "Wat komt er op?/Wat gaat er door je heen?/Wat merk je?"

(geen dialog beginnen!)....

"Concentreer je daarop/Ga daar mee door"

$<<<<$ set met afleidende stimulus $\gg>>>$

Doorgaan totdat zich geen nieuwe veranderingen meer aandienen ('einde van een associatieketen'). Ga in principe iedere 5-10 minuten 'back to target'. 
Back to target

a. Test SUD. "Haal het beeld waarmee we zijn begonnen weer voor je, zoals het nu in je hoofd ligt opgeslagen...hoeveel spanning roept dat beeld nu op, op een schaal van 0 tot 10 , waarbij 0 betekent helemaal geen spanning of helemaal neutraal, en 10 betekent maximale spanning?" (ZONDER DAT DE NC WORDT GEBRUIKT!)

Als SUD > 0:

b. "Welk aspect van dat beeld veroorzaakt die spanning op dit moment het meest?" (Eventueel het cijfer noemen, bijvoorbeeld: "Wat veroorzaakt met name nog die 4?") Wat zie je?

c. "Concentreer je op dat aspect...Ja, heb je dat?"

$\ll<<$ set met afleidende stimulus $\gg \gg>>$ (totdat er geen verandering meer is, en in principe binnen 5-10 minuten, terug naar a: 'back to target').

Herhaal de 'back to target'-procedure tot SUD $=0$ (target) is bereikt. Indien SUD = 0 (target):

"Weet je absoluut zeker dat er niet ergens nog een heel klein restje spanning opkomt als je kijkt naar het beeld? ... Span je eens in om je te laten raken..."

Zo nodig (dwz. SUD > 0) desensitizatie vervolgen.

Zodra SUD = 0 op het plaatje: Check het hele 'filmpje' of 'fotoboek' om te controleren of er echt geen plaatjes in de herinnering meer zijn die spanning oproepen":

"Je geeft aan dat dit beeld nu geen spanning meer oproept. Nu vraag ik je om met gesloten ogen de gehele herinnering, zoals die nu in je hoofd ligt opgeslagen, nog een keer te bekijken. Zijn er nog plaatjes van deze herinnering die nu toch nog spanning oproepen?"

Als dit het geval is, vraag dan wat de patient ziet, hoeveel spanning het nu nog geeft, en welk aspect van dit beeld die spanning oproept (analoog aan de 'back to target'-procedure), en 


\section{Appendices}

desensitiseer ook dit beeld tot SUD $=0$. Herhaal deze stappen voor elk nog spanningsvolle beeld, totdat de gehele herinnering neutraal aanvoelt. Ga dan door met de installatiefase (stap 4) en body scan (stap 5) met betrekking tot het eerst gekozen beeld.

Als de sessie niet afgerond kan worden met SUD = 0, dan positief afsluiten (stap 6).

\section{Installatie van de PC}

a. Test VoC (PC + beeld).

"Neem het oorspronkelijke beeld, dat wil zeggenhet beeld waarmee we zijn begonnen, zoals het nu in je hoofd ligt opgeslagen, in gedachten, en zeg in gedachten...[PC]...". Op een schaal van 1 (volledig onwaar) tot en met 7 (volledig waar), hoe geloofwaardig voelt die uitspraak dan aan?"

b. Instructies (ook als VoC direct al 7 is):

"Kijk naar het beeld en zeg in gedachten ...[PC].... Ja, heb je dat?

c. Set met afleidende stimulus.

Niet vragen naar associaties! Ga direct verder met a. Blijf doorgaan ( $a, b, \&$ c) tot en met een VoC van 7.

5. Body scan (Alleen als je nog voldoende tijd over hebt in de zitting)

a. "Sluit je ogen, neem het beeld [eventueel werktitel noemen] in gedachten, zeg in jezelf ... $[P C] \ldots$ en loop met aandacht je hele lichaam door, van top tot teen en neem waar of er nog ergens lichamelijke spanning bij dit beeld opkomt"

b. In geval van spanning: Set met afleidende stimulus en vraag "Wat komt er in je op?” Vervolg dan met het volgen van associaties (voortgezette desensitisatie).

Als zich geen nieuwe associaties meer aandienen: doorgaan met a) en b) tot de spanning weg is en/of er geen nieuwe associaties meer zijn.

Indien is vastgesteld dat sprake is van angstaanjagende beelden met betrekking tot gevreesde gebeurtenissen, en zeker in geval van vermijdingsgedrag of veiligheidsgedrag vanwege anticipatieangst, desensitiseer dan deze zogenoemde flashforward (zie Bijlage 1) en doe indien zinvol - de mental video check met future template (zie Bijlage 2).

6. Positief afsluiten (aan het eind van iedere sessie; staat los van target)

a. "Wat is het meest positieve of waardevolle dat je het afgelopen uur over jezelf hebt geleerd 
(of hebt ervaren) met betrekking tot ... (maak keuze: dit thema, deze herinnering; de afgelopen sessie)? Indien zinvol: "Wat zegt dat over jou zelf (als persoon)?" of "Hoe noem je zo iemand?" b. Eventueel: "Ga eens zitten als iemand die ... is"

c. "Concentreer je hierop". Gedurende 10 seconde laten concentreren op de positieve zelfspraak, de daarbij horende lichaamshouding en mimiek.

d. Na circa 10 seconden concentreren: "Is er nog iets anders positiefs dat opkomt?"

e. Doorgaan als er nog iets positiefs opkomt; dwz. Opnieuw 10 seconden concentreren op de positieve associatie totdat er geen verdere positieve veranderingen meer optreden.

Uitleg over komende (drie) dagen, afspraken: dagboek, bereikbaarheid, etc.

\section{Volgende zitting}

a. Indien de SUD > 0 bij afsluiten van de vorige sessie: check de SUD bij aanvang van de sessie. Maak een keuze uit de volgende opties:

- Indien SUD bij aanvang van sessie > 0: start de desensitisatie op met back to target (dus zonder NC te noemen)

- Indien SUD target = 0: check het hele 'filmpje' of 'fotoboek' om te controleren of er echt geen plaatjes in de herinnering meer zijn die spanning oproepen.

- Indien de SUD van alle targets uit de herinnering 0 is, en de VoC van de PC van het eerstgekozen target is7, zijn er de volgende opties:

- rond de behandeling af als het beoogde resultaat van de therapie is bereikt, of

- ga oor met (een) eventuele volgende herinnering(en) die in de casusconceptualisatie zijn opgenomen, of

- $\quad$ stel het behandelplan bij op grond van het bereikte resultaat en vervolg de behandeling.

\section{Bijlage 1. Flashforward}

In het geval de patiënt anticipatie-angst heeft (en dus vermijdingsreacies vertoont) ten aanzien van objecten of situaties in de toekomst, indentificeer dan de rampfantasie van die persoon over de toekomst, iets wat hem of haar zal overkomen ('het ultieme schrikbeeld') bij een confrontatie met het/de gevreesde object/situatie.

Benoem het doel van de interventie.

"Ik vermoed dat jouw angst voor ....(noem object of situatie) ..zo sterk is vanwege een schrikbeeld of rampfantasie in je hoofd over deze situatie. Waar we nu naar toe werken 


\section{Appendices}

is dat je dit schrikbeeld aan kunt, dat wil zeggen dat je er rustig naar kunt kijken en dat je beseft dat het niet waarschijnlijk is dat de ramp, zoals jij die in je hoofd hebt, daadwerkelijk op deze manier gaat gebeuren. De verwachting is dat je angst dan minder zal worden."

- Identificeer de flashforward.

"Wij gaan nu uitzoeken welke beelden je in je hoofd hebt van wat je vreest dat er zal gebeuren - of eigenlijk zal misgaan-, wanneer je in contact komt met .......... (noem object of situatie).

We zoeken dus naar 'het ultieme schrikbeeld' dat je nu verhindert om te doen wat je wilt doen. Je mag je ogen sluiten om je beter te concentreren....Wat denk je dat er misgaat? ... Welke ramp zal zich voor gaan doen? ..."Maak hiervan een stilstaand plaatje". Welke beeld heb jij? ... Hoe zit dat schrikbeeld precies in je hoofd?"

- Stel eventueel aanvullende vragen als:

"Wat stel je je precies voor dat er mis kan gaan als je ..."

"Als je hierover een nachtmerrie zou hebben ...waar zou die dan over gaan?"

Check of er niet nog een 'ramp achter de ramp' ligt bijvoorbeeld: "Wat zou er kunnen gebeuren als je een paniekaanval krijgt? of "....als je een auto-ongeval krijgt".

- Desensitiseer de flashforward methet EMDR-standaardprotocol als ware het een herinnering. Start hierbij in het standaardprotocol bij 2.5 (vragen naar de emotie), en gebruik daarvoor de NC/PC combinatie ("Ik ben machteloos"/"Ik kan het aan") dan wel een andere SUDverhogende uitspraak. Ga door tot SUD $=0$ en $\mathrm{VoC}=7$.

- Emotie

"Als je het beeld weer in gedachten neemt en tegelijkertijd in jezelf zegt 'ik ben machteloos', welke emotie voel je dan nu, op dit moment?" Noem eventueel bij wijze van hulp: 'bijvoorbeeld bang, boos, bedroefd'. 
- $\quad$ Lading van het beeld (SUD 0-10)

"Neem het beeld in gedachten en zeg in jezelf 'ik ben machteloos' (of een andere SUDverhogende uitspraak2), hoeveel spanning voel je dan nu, op dit moment, geschat op een schaal lopend van 0 , helemaal geen spanning, tot en met 10 , maximale spanning?"

- $\quad$ Plaats van de spanning

"Waar in je lichaam voel je dat ('die spanning') het sterkst?"

\section{- Desensitisatie}

Bij gebruik van oog-/handbewegingen: Hand klaar voor de ogen:

"Kijk naar (de toppen van) mijn vingers."

a. Neem het beeld in gedachten, voel jouw machteloosheid ten opzichte van dit beeld "...Wees je bewust van de spanning in je...[locatie van de spanning]".

Geef even de tijd zich te concentreren.

"Volg mijn hand en stel je open voor wat er daarna spontaan bij je opkomt"

$\ll<<$ Set met afleidende stimulus $\gg \gg \gg$ (circa een halve minuut).

b). "Wat komt er op"|"Wat gaat er door je heen"/"Wat merk je?"

(geen dialoog beginnen!)...

"Concentreer je daar op"|"Ga daar mee door."

$<<<<$ Set met afleidende stimulus $>>>>$

Doorgaan totdat er zich geen nieuwe veranderingen meer aandienen ('einde van een associatieketen'). Ga in principe iedere 5 -10 minuten 'back to target'.

- $\quad$ Back to target

a. Test SUD. "Haal het beeld waarmee we zijn begonnen weer voor je, zoals het nu in je 


\section{Appendices}

hoofd ligt opgeslagen.....hoeveel spanning roept dat nu op, op een schaal van 0 tot 10, waarbij 0 betekent helemaal geen spanning of helemaal neutraal, en 10 betekent maximale spanning)?" (ZONDER DAT DE NC WORDT GEBRUIKT!).

Indien SUD > 0:

b). "Welk aspect van dat beeld veroorzaakt die spanning op dit moment het meest?" (Eventueel het cijfer noemen, bijvoorbeeld: "Wat veroorzaakt met name nog die 4?...")

Wat zie je?

c). "Concentreer je op dat aspect......Ja, heb je dat?"

$<<<$ Set met afleidende stimulus $\gg>>$ (totdat er geen verandering meer is, en in principe binnen 10 minuten, terug naar a: 'back to target'.)

Herhaal de 'back to target'-procedure tot SUD = 0 (target) is bereikt.

Indien SUD = 0 (target):

"Weet je absoluut zeker dat er niet ergens nog een heel klein restje spanning zit?...... Span je eens in om je te laten raken......"

Zo nodig desensitisatie vervolgen tot SUD $=0$.

\section{- Installatie van de PC}

a. Test VoC (met PC + beeld)

"Neem het oorspronkelijke beeld, dat wil zeggen het beeld waarmee we zijn begonnen, zoals het nu in je hoofd ligt opgeslagen, in gedachten, en zeg in jezelf 'ik kan het aan'.... Op een schaal van 1 (volledig onwaar) tot en met 7 (volledig waar), hoe geloofwaardig voelt die uitspraak dan aan?"

b). Instructies (ook als VoC direct al 7 is):

"Kijk naar het beeld en zeg in jezelf 'ik kan het aan'......Ja, heb je dat?"

c). Set met afleidende stimulus.

Niet vragen naar associaties! Ga direct verder met a. Blijf doorgaan ( $a, b$ \& c) net zo lang totdat de VoC 7 is geworden. 
Herhaal de procedure voor eventuele andere aanwezige flashforwards.

\section{Bijlage 2. Mental Video Check}

Om na te gaan of er aspecten/'cues' zijn die zodanig veel spanning oproepen dat ze de patiënt zouden kunnen verhinderen om de toekomstige situatie aan te gaan laat je de patiënt in verbeelding, en met gesloten ogen, door een wenselijke toekomstige situatie gaan (vanaf het begin tot en met het einde, waarbij moet worden opgelet dat de patiënt niet vermijdt). De patiënt vertelt daarbij hardop wat hij/zij ziet.

- Vraag de patiënt de ogen te openen op het moment dat spanning wordt waargenomen en zich daarbij te focussen op dat aspect dat de meeste spanning veroorzaakt. Voer direct een set uit en ga daarna door met deze procedure tot de hele film zonder spanning kan worden bekeken.

"Ik zou je willen vragen om straks je ogen dicht te doen en een situatie in gedachten te nemen die je tot nu toe moeilijk of spannend vindt om mee te maken. Welke situatie is dat? ... Stap zo direct denkbeeldig in die situatie en vertel wat je ziet. Je bent dan als het ware al in de toekomst en je maakt het zelf mee. Begin bij het begin en loop door de situatie heen tot het einde, terwijl je nagaat of er ergens spanning optreedt. Vertel me dan hardop wat je meemaakt. Op het moment dat je spanning ervaart, concentreer je dan op het meest spannende aspect van die situatie en open je ogen. Dat is voor mij het teken dat je een moeilijk of spannend moment in je hoofd hebt en dan volg je weer mijn hand met je ogen. Is dat oké?"...

"Nog even voor de zekerheid: zodra je spanning opmerkt concentreer je dan op wat je meemaakt en op wat het zo spannend maakt. Open dan je ogen, en volg mijn hand...... Begin maar en vertel wat je ervaart."

$\ll<<$ Set met afleidende stimulus $\gg \gg>$ (circa een halve minuut; niet vragen naar associaties).

"Loop de film nu verder door en als je weer spanning ervaart, concentreer je dan weer op dat wat het spannend maakt en open dan je ogen ..." 


\section{Appendices}

De mental video-check wordt net zo vaak herhaald (eventueel, na een aantal rapportages, niet meer hardop maar in gedachten) totdat deze door de patiënt zonder enige spanning wordt doorlopen.

Check of de patiënt nu verwacht de situatie te kunnen aangaan.

"Neem een stilstaand beeld van de toekomstige situatie in gedachten, op een schaal van 1 tot en met 7, in welke mate kan je het nu aan om het ook echt te doen?"

Als dat nog niet (voldoende) het geval is, ga dan verder met de volgende stap.

\section{- Future template}

"Neem opnieuw dat stilstaande beeld van de toekomstige situatie in gedachten, en zeg in jezelf: 'Ik kan dit aan', ...Ja, heb je dat?"

$\ll<<$ Set met afleidende stimulus $\gg \gg>>$ (circa halve minuut; niet vragen naar associaties).

Herhaal deze procedure net zo lang totdat de geloofwaardigheid van de Future template niet verder meer stijgt (analoog aan installatie van de PC).

\section{- Huiswerkopdrachten}

- Bereid samen met de patiënt gedragsexperimenten/exposure-opdrachten voor om ervoor te zorgen dat de patiënt het vertrouwen in de (voorheen) angstwekkende situatie verder, op eigen kracht, kan herstellen.

- Laat de patiënt (als huiswerkopdracht) de proef op de som nemen.

- Evalueer het resultaat van het experiment en plan, indien nodig, opnieuw een gedragsexperiment/exposure-opdracht. 


\section{APPENDIX 2 - UNWANTED EVENT TO ADVERSE \\ TREATMENT REACTION (UE-ATR) CHECKLIST}

Linden, 2013

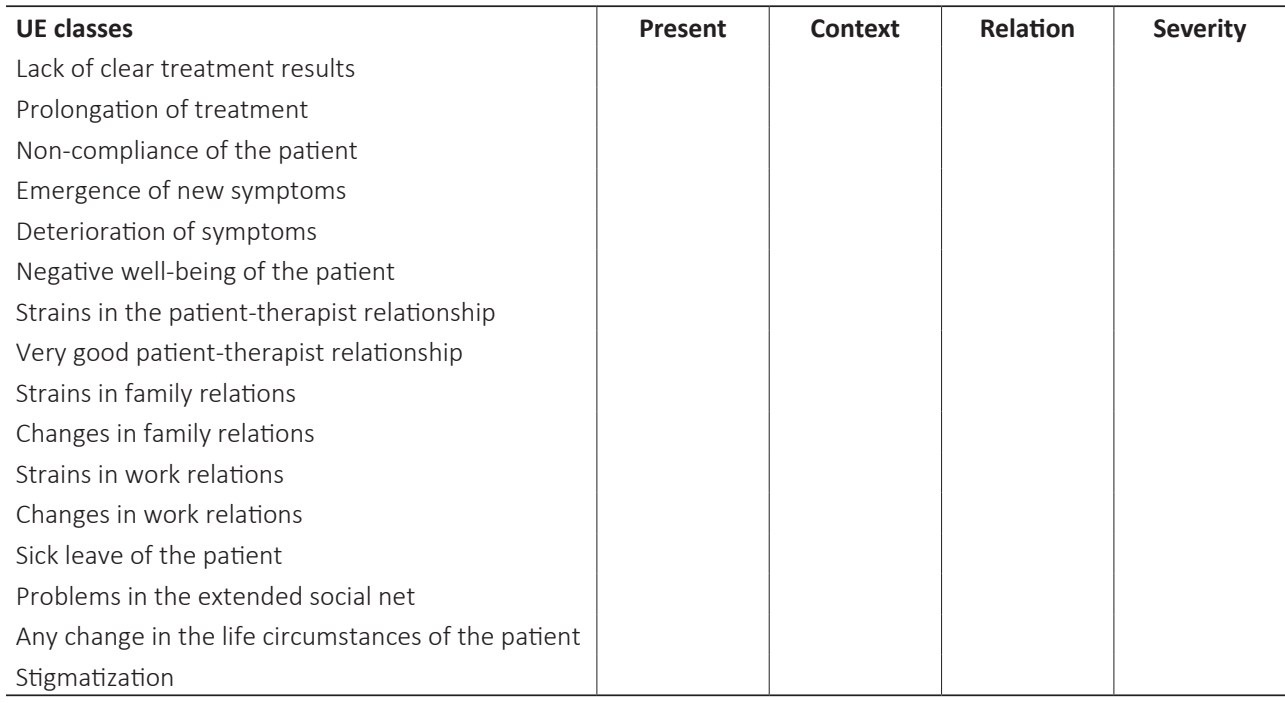

\section{Glossary of ratings}

\begin{tabular}{|c|c|c|}
\hline Context of development & Relation to treatment & Severity \\
\hline 1. Diagnostic procedures & 1. Unrelated & 1. Mild, without consequences \\
\hline Theoretical orientations & 2. Probably unrelated & 2. Moderate, distressing \\
\hline Selection of the treatment focus & 3. Possibly related & 3. Sever, in need of countermeasures \\
\hline 4. Treatment procedures & 4. Probably related & $\begin{array}{l}\text { 4. Very severe, lasting negative } \\
\text { consequences }\end{array}$ \\
\hline Sensitization processes & 5. Related & $\begin{array}{l}\text { 5. Extremely severe, hospitalization } \\
\text { required, or life threatening }\end{array}$ \\
\hline Disinhibition processes & & \\
\hline 7. Treatment effects & & \\
\hline 8. Therapist-patient relationship & & \\
\hline
\end{tabular}


Appendices

\section{APPENDIX 3 - NEGATIVE EVENTS QUESTIONNAIRE (NEQ)}

Rozental et al., 2016

Incidents and effects that are viewed as both positive and negative can occur during treatment. We now want you to think about what happened during the period when you received your treatment, and that you consider if anything you experienced was negative or unwanted. Read through the following statements and mark whether you experienced any of these incidents or effects. If you answer yes, please indicate the severity of your experience (how negative the experience was for you), and whether you believe your experience was caused by the treatment you received or other circumstances that occurred during the same period as your treatment.

\begin{tabular}{|c|c|c|c|c|c|c|c|c|c|}
\hline Incidents and effects & \multicolumn{2}{|c|}{$\begin{array}{l}\text { Did you } \\
\text { experience } \\
\text { this? }\end{array}$} & \multicolumn{5}{|c|}{$\begin{array}{l}\text { If yes - here is how } \\
\text { negatively it affected } \\
\text { me }\end{array}$} & \multicolumn{2}{|c|}{ Probably caused by } \\
\hline & No & Yes & $\begin{array}{l}\overline{\overline{0}} \\
+\pi \\
+ \\
+ \\
\dot{0}\end{array}$ & 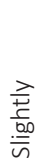 & $\begin{array}{l}\frac{\lambda}{0} \\
\frac{0}{0} \\
\frac{0}{0} \\
\frac{0}{0} \\
\frac{0}{2}\end{array}$ & $\frac{3}{d}$ & 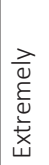 & $\begin{array}{l}\text { The treatment } \\
\text { I have received }\end{array}$ & $\begin{array}{l}\text { Other } \\
\text { circumstances }\end{array}$ \\
\hline 1. I had more problems with my sleep & & & & & & & & & \\
\hline 2. I felt like I was under more stress & & & & & & & & & \\
\hline 3. I experienced more anxiety & & & & & & & & & \\
\hline 4. I felt more worried & & & & & & & & & \\
\hline 5. I felt more dejected & & & & & & & & & \\
\hline 6. I experienced more hopelessness & & & & & & & & & \\
\hline 7. I experienced lower self-esteem & & & & & & & & & \\
\hline 8. I lost faith in myself & & & & & & & & & \\
\hline 9. I felt sadder & & & & & & & & & \\
\hline 10. I felt less competent & & & & & & & & & \\
\hline $\begin{array}{l}\text { 11. I experienced more unpleasant } \\
\text { feelings }\end{array}$ & & & & & & & & & \\
\hline $\begin{array}{l}\text { 12. I felt that the issue I was looking for } \\
\text { help with got worse }\end{array}$ & & & & & & & & & \\
\hline 13. Unpleasant memories resurfaced & & & & & & & & & \\
\hline $\begin{array}{l}\text { 14. I became afraid that other people } \\
\text { would find out about my treatment }\end{array}$ & & & & & & & & & \\
\hline $\begin{array}{l}\text { 15. I got thoughts that it would be better } \\
\text { if I did not exist anymore and that I } \\
\text { should take my own life }\end{array}$ & & & & & & & & & \\
\hline $\begin{array}{l}\text { 16. I started feeling ashamed in front of } \\
\text { other people because I was having } \\
\text { treatment }\end{array}$ & & & & & & & & & \\
\hline
\end{tabular}


17. I stopped thinking that things could get better

18. I started thinking that the issue I was seeking help for could not be made any better

19. I started thinking help was possible

20. I think that I have developed a dependency on my treatment

21. I think that I have developed a dependency on my treatment

22. I did not always understand my treatment

23. I did not always understand my therapist

24. I did not have confidence in my treatment

25. I did not have confidence in my therapist

26. I felt that the treatment did not produce any results

27. I felt that my expectations for the treatment were not fulfilled

28. I felt that my expectations for the therapist were not fulfilled

29. I felt that the quality of the treatment was poor

30. I felt that the treatment did not suit me

31. I felt that I did not form a closer relationship with my therapist

32. I felt that the treatment was not motivating

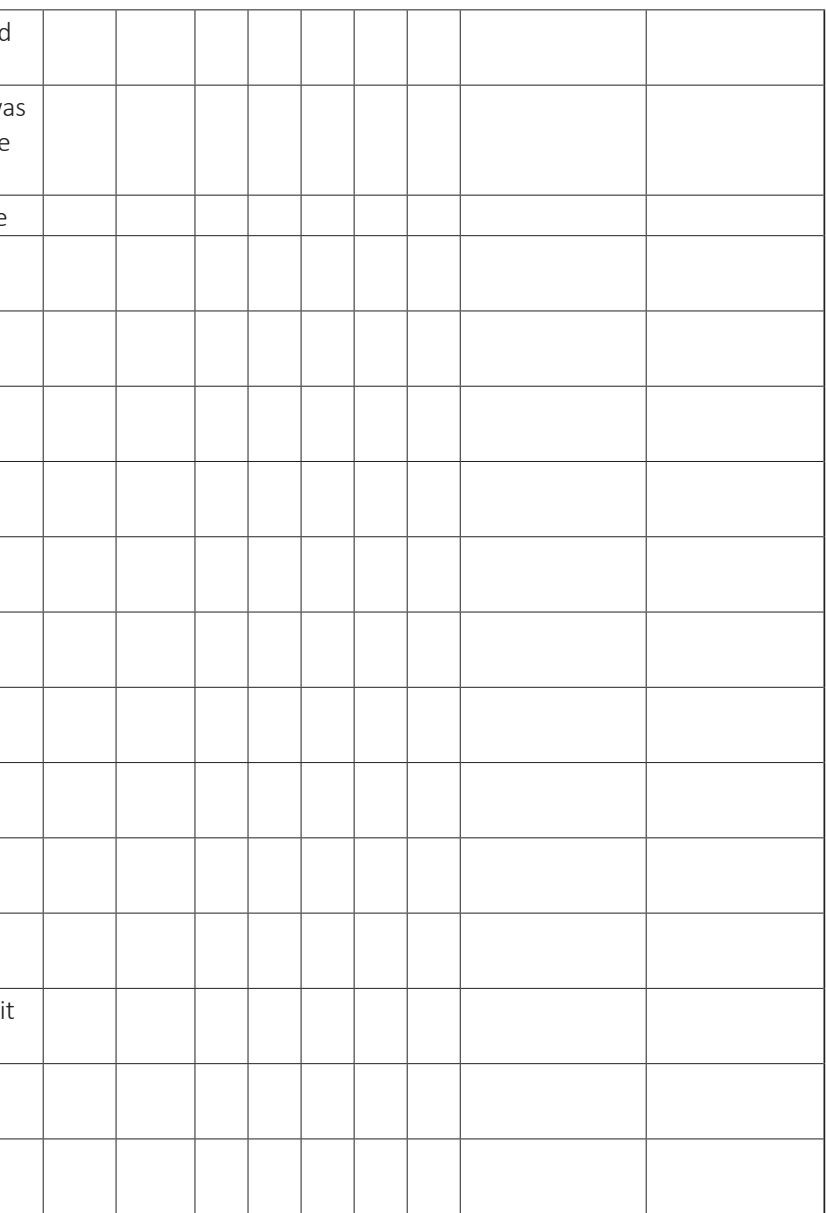




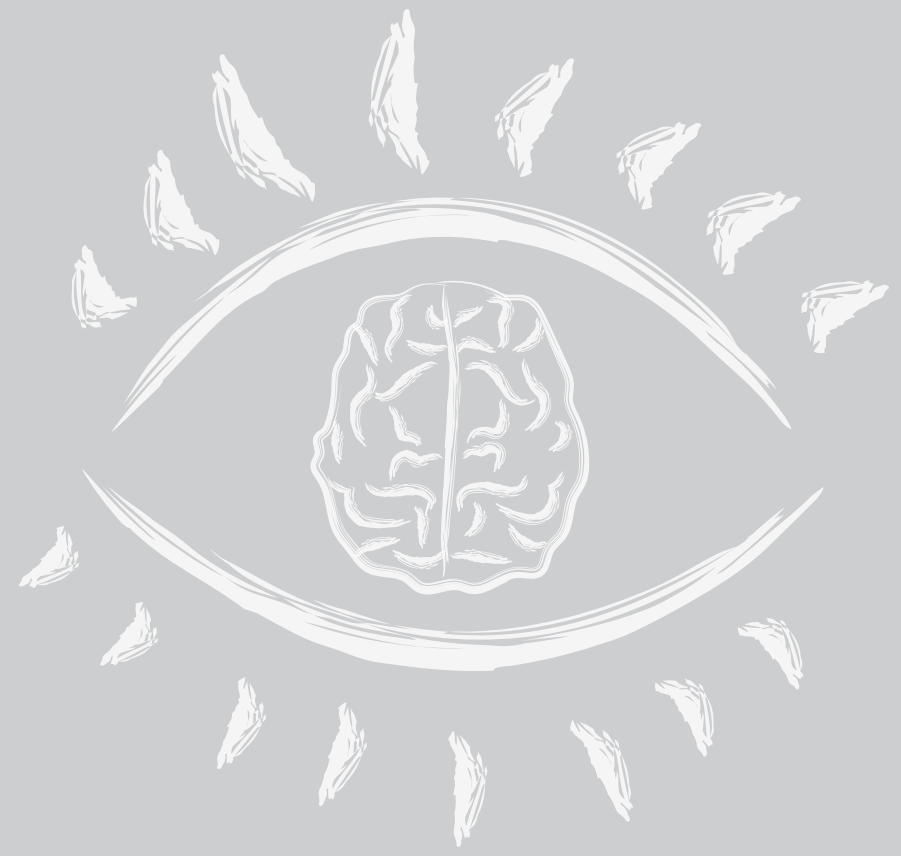


References 

References marked with an asterisk indicate studies included in the meta-analysis of Chapter 3.

Akhtar, S., Justice, L. V., Knott, L., Kibowski, F., \& Conway, M. A. (2018). The 'common sense' memory belief system and its implications. International Journal of Evidence \& Proof, 22(3), 289-304. https://doi.org/10.1177/1365715718784045

Allen, T. M. (2004). Efficacy of EMDR and chronic pain management. Chicago: Argosy University.

American Psychiatric Association (2013). Diagnostic and statistical manual of mental disorders ( $5^{\text {th }}$ ed.). Washington, DC: American Psychiatric Association.

Anderson, M. C., \& Green, C. (2001). Suppressing unwanted memories by executive control. Nature, 410, 366-369. https://doi.org/10.1038/35066572

Andrade J, Kavanagh D, \& Baddeley A. (1997). Eye-movements and visual imagery: A working memory approach to the treatment of post-traumatic stress disorder. British Journal of Clinical Psychology, 36(2), 209-23. https://doi.org/10.1111/j.2044-8260.1997.tb01408.x

Artnz, A. (2012). Imagery rescripting as a therapeutic technique: Review of clinical trials, basic studies, and research agenda. Journal of Experimental Psychopathology, 3(2), 189-208. https://doi.org/10.5127/jep.024211

Baddeley, A. D. (1998). Human memory: Theory and practice. Needham Heights, MA: Allyn \& Bacon. Balbo, M., Zaccagnino, M., Cussino, M., \& Civilotti, C. (2017). Eye movement desensitization and reprocessing (EMDR) and eating disorders: A systematic review. Clinical Neuropsychiatry, 14(5), 321-329. https://doi.org/10.1002/(SICI)1098-108X(199801)23:1<1::AID-EAT1>3.0.CO;2-Q

Balota, D. A., Cortese, M. J., Duchek, J. M., Adams, D., Roediger, H. L., McDermott, K. B., \& Yerys, B. E. (1999). Veridical and false memory in healthy older adults and in Dementia of the Alzheimer's type. Cognitive Neuropsychology, 16(3-5),361-384. https://doi.org/10.1080/026432999380834

Barden, R. C. (2016). Memory and reliability: Developments and controversial issues. In P. Radcliffe, A. Heaton-Armstrong, G. Gudjonsson, D. Wolchover (Eds.), Witness testimony in sex cases (pp. 343-359). Oxford, England: Oxford University Press.

*Barrowcliff, A. L., Gray, N. S., Freeman, T. C. A., \& Macculloch, M. J. (2004). Eye-movements reduce the vividness, emotional valence and electrodermal arousal associated with negative autobiographical memories. The Journal of Forensic Psychiatry \& Psychology, 15(2), 325-345. https://doi.org/10.1080/14789940410001673042

Bauman, W., \& Melnyk, W. T. (1994). A controlled comparison of eye movement and finger tapping in the treatment pf test anxiety. Journal of Behavior Therapy and Experimental Psychiatry, 25(1), 29-33. https://doi.org.10.1016/0005-7916(94)90060-4 


\section{References}

Beck, A. T., \& Steer, R. A. (1987). Beck Depression Inventory: Manual. San Antonio, TX: Psychiatric Corporation.

Beer, R., ten Broeke, E., Hornsveld, de Jongh, A., Meijer, S., de Roos, C., et al. (2011). EMDR: oogbewegingen of een andere duale taak? [EMDR: Eye movements or another dual task?]. http://www.emdr.nl/wp-content/uploads/2016/01/EMDR-Richtlijnendoor- trainers.pdf Behnammoghadam, M., Alamdari, A. K., Behnammoghadam, A., \& Darban, F. (2015). Effect of Eye Movement Desensitization and Reprocessing (EMDR) on Depression in Patients with Myocardial Infarction (MI). Global Journal of Health Science, 7(6), 258-262. http://dx.doi.org/10.5539/gjhs.v7n6p258

Behnammoghadam, M., Behnammoghadam, A., \& Salehian, T. (2015). Efficacy of Eye Movement Desensitization and Reprocessing (EMDR) on depression in patients with Myocardial Infarction (MI) in a 12-month follow up. Iranian Journal of Critical Care Nursing, 8(4), 221-226.

Belli, R. F., \& Loftus, E. F. (1994). Recovered memories of childhood abuse: A source monitoring perspective. In S. J. Lynn, \& J. Rhue (Eds.), Dissociation: Theory, clinical, and research perspectives (pp. 415-433). New York: Guilford.

Benjet, C., Bromet, E., Karam, E. G., Kessler, R. C., McLaghlin, K. A., Ruscio, A. M., ... \& Alonso, J. (2016). The epidemiology of traumatic event exposure worldwide: Results from the World Mental Health Survey Consortium. Psychological Medicine, 46(2), 327-343. https://doi.org/10.1017/ S0033291715001981

Benner, E. L., \& Lindsay, D. S. (2007). Memory of remembering: Investigating the forgot-it-all-along effect using pictures. Psi Chi Journal of Undergraduate Research, 12(1), 51-58.

van Benthem, P., Spijkerman, R., Blanken, P., Kleinjan, M., Vermeiren, R. R. J. M., Hendriks, V. M. (2020). A dual perspective on first-session therapeutic alliance: Strong predictor of youth mental health and addiction treatment outcome. European Child \& Adolescent Psychiatry, 1-9. https://doi.org/10.1007/s00787-020-01503-w

van den Berg, D. P., de Bont, P. A., van der Vleugel, B., M., de Roos, C., de Jongh, A., van der Gaag, M., et al. (2015). Prolonged exposure vs eye movement desensitization and reprocessing vs waiting list for posttraumatic stress disorder in patients with a psychotic disorder a randomized clinicaltrial.JAMA Psychiatry, 72(3), 259-267. https://doi.org/10.1001/jamapsychiatry.2014.2637

Bernstein, E. M., \& Putnam, F. W. (1986). Development, reliability and validity of a dissociation scale. The Journal of Nervous and Mental Disease, 174(12), 727-735. https://doi.org/10.1097/ 00005053-198612000-00004

Bernstein, D. M., Scoboria, A., Desjarlais, L., \& Souci, K. (2018). "False memory" is a linguistic 
convenience. Psychology of Consciousness: Theory, Research, and Practice, 5(2), 161-179. https://doi.org/10.1037/cns0000148

Bidrose, S., \& Goodman, G. S. (2000). Testimony and evidence: A scientific case study of memory for child sexual abuse. Applied Cognitive Psychology, 14(3), 197-213. https://doi.org/10.1002/ (SICI)1099-0720(200005/06)14:3<197::AID-ACP647>3.0.CO;2-6

Bisson, J., \& Andrew, M. (2008). Psychological treatment of post-traumatic stress disorder (PTSD). Cochrane Database of Systematic Reviews, 3(3), CD003388. https://doi.org/10.1102/ 14651858.CD003388.pub3

Bisson, J. I., Ehlers, A., Mathews, A., Pilling, S., Richards, D, \& Turner, S. (2007). Psychological treatments for chronic post-traumatic stress disorder: systematic review and meta-analysis. British Journal of Psychiatry, 190, 97-104. https://doi.org/10.1192/bjp.bp.106.021402

Blake, D .D., Weathers, F. W., Nagy, L. M., Kaloupek, D .G., Klauminzer, G., Charney, D. S., \& Keane, T. M. (1990). A clinician rating scale for assessing current and lifetime PTSD: The CAPS-1. Behavior Therapist, 13, 187-188

Blank, H., \& Launay, C. (2014). How to protect eyewitness memory against the misinformation effect: A meta-analysis of post-warning studies. Journal of Applied Research in Memory and Cognition, 3(2), 77-88. https://doi.org/10. 1016/j.jarmac.2014.03.005

Bloomgarden, A., \& Calogero, R. M. (2008). A randomized experimental test of the efficacy of EMDR treatment on negative body image in eating disorder inpatients. Eating Disorders, 16(5), 418427. https://doi.org/10.1080/10640260802370598

de Bont, P. A., van den Berg, D. P. G., van der Vleugel, B. M., de Roos, C., de Jongh, A., van der Gaag, M., \& van Minnen, A. (2016). Prolonged exposure and EMDR for PTSD v. a PTSD waiting-list condition: effects on symptoms of psychosis, depression and social functioning in patients with chronic psychotic disorders. Psychological Medicine, 46(11), 2411-2421. https://doi. org/10.1017/S0033291716001094

de Bont, P. A. J. M., van den Berg, D. P. G., van der Vleugel, B. M., de Roos, C., Mulder, C. L., Becker, E. S., de Jongh, A., van der Gaag, M., \& van Minnen, A. (2013). A multi-site single blind clinical study to compare the effects of prolonged exposure, eye movement desensitization and reprocessing and waiting list on patients with a current diagnosis of psychosis and co morbid post traumatic stress disorder: Study protocol for the randomized controlled trial treating trauma in psychosis. Trials, 14, 151. https://doi.org/10.1186/1745-6215-14-151

Bookbinder, S. H., \& Brainerd, C. J. (2016). Emotion and false memory: The context-content paradox. Psychological Bulletin, 142(12), 1315-1351. https://doi.org/10.1037/bul0000077 


\section{References}

Borenstein, M., Higgins, J. P. T., Hedges, L. V., \& Rothstein, H. R. (2017). Basics of meta-analysis: I2 is not an absolute measure of heterogeneity. Research Synthesis Methods, 8(1), 5-18. https://doi.org/10.1002/jrsm.1230

Born, J., Rasch, B., \& Gais, S. (2013). Guidelines for the management of conditions specifically related to stress. Geneva: World Health Organization.

Bourne, C., Frasquilho, F., Roth, A. D., \& Holmes, E. A. (2010). Is it mere distraction? Peri- traumatic verbal tasks can increase analogue flashbacks but reduce voluntary memory performance. Journal of Behaviour Therapy and Experimental Psychiatry, 41(3), 316-324. https://doi.org/10.1016/ j.jbtep.2010.03.001

Bradley, R., Greene, J., Russ, E., Dutra, L., \& Westen, D. (2005). A multidimensional meta-analysis of psychotherapy for PTSD. The American Journal of Psychiatry, 162(2), 214-227. https://doi.org/10.1176/appi.ajp.162.2.214

Bradley, M. M., Greenwald, M. K., Petry, M. C., \& Lang, P. J. (1992). Remembering pictures: Pleasure and arousal in memory. Journal of Experimental Psychology: Learning, Memory, and Cognition, 18(2), 379-390. https://doi.org/10.1037/0278-7393.18.2.379

Brainerd, C. J., \& Reyna, V. F. (2002). Fuzzy-trace theory and false memory. Current Directions in Psychological Science, 11(5), 164-169. https://doi.org/10.1111/1467-8721.00192

Brainerd, C. J., \& Reyna, V. F. (2005). The Science of False Memory. New York, NY: Oxford University Press.

Brainerd, C. J., Holliday, R. E., Reyna, V. F., Yang, Y., \& Toglia, M. P. (2010). Developmental reversals in false memory: Effects of emotional valence and arousal. Journal of Experimental Child Psychology, 107(2), 137-154. https://doi.org/10.1016/j.jecp.2010.04.013

Brainerd, C. J., Reyna, V. F., \& Ceci, S. J. (2008). Developmental reversals in false memory: A review of data and theory. Psychological Bulletin, 134(3), 343-382. https://doi.org/10.1037/00332909.134.3.343

Brenneis, C. B. (2000). Evaluating the evidence: Can we find authenticated recovered memory? Psychoanalytic Psychology, 17(1), 61-77. https://doi.org/10.1037/0736-9735.17.1.61

Brewin, C. R., \& Holmes, E. A. (2003). Psychological theories of posttraumatic stress disorder. Clinical Psychology Review, 23(3), 339-376. https://doi.org/10.1016/S0272-7358(03)00033-3

Brewin, C. R., Dalgleish, T., \& Joseph, S. (1996). A dual representation theory of posttraumatic stress disorder. Psychological Review, 103(4), 670-686. https://doi.org/10.1037/0033295X.103.4.670

Brewin, C. R., Gregory, J. D., Lipton, M., \& Burgess, N. (2010). Intrusive images in psychological disorders: characteristics, neural mechanisms, and treatment implications. Psychological Review, 117(1), 
210e232. https://doi.org/10.1037/a0018113

Brewin, C. R., Li, H., Ntarantana, V., Unsworth, C., \& McNeilis, J. (2019). Is the public understanding of memory prone to widespread "myths"? Journal of Experimental Psychology: General, 148(12), 2245-2257. https://doi.org/10.1037/xge0000610

Briere, J., \& Conte, J. (1993). Self-reported amnesia for abuse in adults molested as children. Journal of Traumatic Stress, 6(1), 21-31. https://doi.org/10.1002/jts.2490060104

Brown, R., \& Kulik, J. (1977). Flashbulb memories. Cognition, 5(1), 73-99. https://doi.org/10.1016/ 0010-0277(77)90018-X

Brown, K. W., McGoldrick, T., \& Buchanan, R. (1997). Body dysmorphic disorder: Seven cases treated with eye movement desensitization and reprocessing. Behavioural and Cognitive Psychotherapy, 25 (2), 203-207. https://doi.org/10.1017/S1352465800018403

Brown, D., Scheflin, A. W., \& Whitfield, C. L. (1999). Recovered memories: The current weight of the evidence in science and in the courts. Journal of Psychiatry and Law, 27(1), 6-156. https://doi.org/10.1177/009318539902700102

Buckley, T. C., Blanchard, E. B., \& Neill, W. T. (2000). Information processing and PTSD: a review of the empirical literature. Clinical Psychology Review, 20(8), 1041 -1065. https://doi.org/10.1016/ S0272-7358(99)0030-6

Bulevich, J. B., Roediger III, H., Balota, D. A., \& Butler, A. C. (2006). Failures to find suppression of episodic memories in the think/no-think paradigm. Memory \& Cognition, 34(8), 1569-1577. https://doi.org/10.3758/BF03195920

Butler, K. M., McDaniel, M. A., Dornburg, C. C., Price, A. L., \& Roediger, H. L. (2004). Age differences in veridical and false recall are not inevitable: The role of frontal lobe function. Psychonomic Bulletin \& Review, 11(5), 921-925. https://doi.org/10.3758/BF03196722

Cahill, L., \& McGaugh, J. L. (1995). A novel demonstration of enhanced memory associated with emotional arousal. Consciousness and Cognition, 4(4), 410 -421. https://doi.org/ 10.1006/ccog.1995.1048

Carletto, S., Ostacoli, L., Colombi, N., Calorio, L., Oliva, F., Fernandez, I., \& Hofmann, A. (2017). EMDR for depression: A systematic review of controlled studies. Clinical Neuropsychiatry, 14(5), 306-312. https://doi.org/10.1002/14651858

Cavillo, D. P., \& Emami, A. S. (2019). Do lateral eye movements increase susceptibility to misinformation? A registered replication. Psychonomic Bulleting \& Review, 26, 1905-1910. https://doi.org/ 10.3758/s13423-019-01641-6

Cann, D. R., \& Katz, A. N. (2005). Habitual acceptance of misinformation: Examination of individual differences and source attributions. Memory and Cognition, 33, 405-417. 


\section{References}

https://doi.org/10.3758/BF03193059

Carter, E. C., Schönbrodt, F. D., Gervais, W. M., \& Hilgard, J. (2019). Correcting for bias in psychology: A comparison of meta-analytic methods. Advances in Methods and Practices in Psychological Science, 2(2), 115-144. https://doi.org/10.1177/2515245919847196

Ceci, S. J., \& Bruck, M. (1993). The suggestibility of the child witness: A historical and synthesis. Psychological Bulletin, 113(3), 403-439. https://doi.org/10.1037/0033-2902.113.3.403

Ceci, S. J., \& Loftus, E. F. (1994). 'Memory work': A royal road to false memories? Applied Cognitive Psychology, 8(4), 351-364. https://doi.org/10.1002/acp.2350080405

Chemali, Z., \& Meadows, M. E. (2004). The use of eye movement desensitization and reprocessing in the treatment of psychogenic seizures. Epilepsy and Behavior, 5(5), 784-787. https://doi.org/ 10.1016/j.yehbeh.2004.06.003

Chen, R., Gillespie, A., Zhao, Y., Xi, Y., Ren, Y., \& McLean, L. (2018). The efficacy of eye movement desensitization and reprocessing in children and adults who have experienced complex childhood trauma: A systematic review of randomized controlled trials. Frontiers in Psychology, 9, 534. https://doi.org/10.3389/fpsyg.2018.00534

Chen, L., Zhang, G., Hu, M., \& Liang, X. (2015). Eye movement desensitization and reprocessing versus cognitive-behavioral therapy for adult posttraumatic stress disorder. The Journal of Nervous and Mental Disease, 203(6), 443-451. https://doi.org/10.1097/NMD.0000000000000306

Christianson, S.-A., \& Loftus, E. F. (1991). Remembering emotional events: The fate of detailed information. Cognition \&Emotion, 5(2),81-108.https://doi.org/10.1080/02699939108411027

Christman, S. D., Garvey, K. J., Propper, R. E., \& Phaneuf, K. A. (2003). Bilateral eye movements enhance the retrieval of episodic memories. Neuropsychology, 17(2), 221-229. https://doi.org/ 10.1037/0894-4105.17.2.221

Christman, S. D., Propper, R. E., \& Dion, A. (2004). Increased hemispheric interaction is associated with decreased false memories in a verbal converging semantic associates paradigm. Brain and Cognition, 56(3), 313-319. https://doi.org/10.1016/j.bandc.2004.08.005

Clancy, S. (2010). The trauma myth: The truth about the sexual abuse of children - and its aftermath. New York, NY: Basic Books.

Clark, S. E., Benjamin, A. S., Wixted, J. T., Mickes, L., Gronlund, S. D. (2015). Eyewitness identification and the accuracy of the criminal justice system. Policy Insights from the Behavioral and Brain Sciences, 2(1), 175-186. https://doi.org/10.1177/2372732215602267

Cochran, K. J., Greenspan, R. L., Bogart, D. F., \& Loftus, E. F. (2016). Memory blindness: Altered memory reports lead to distortion in eyewitness memory. Memory \& Cognition, 44, 717-726. 
https://doi.org/10.3758/s13421-016-0594-y

Cohen, J. (1988). Statistical power analysis for the behavioral sciences, $2^{\text {nd }}$ ed. Hillsdale, NJ: Erlbaum.

Cohen, J. A., Mannarino, A. P., \& Deblinger, E. (2017). Treating trauma and traumatic grief in children and adolescents ( $2^{\text {nd }}$ ed.). New York, NY: Guilford Press.

Collins, A. M., \& Loftus, E. F. (1975). A spreading-activation theory of semantic processing. Psychological Review, 82(6), 407-428. https://doi.org/10.1037/0033-295X.82.6.407

Collins, A. M., \& Quillian, M. R. (1969). Retrieval time from semantic memory. Journal of Verbal Learning and Verbal Behavior, 8(2), 240 -247. https://doi.org/10.1016/S0022-5371(69)80069-1

Cook-Vienot, R., \& Taylor, R. J. (2012). Comparison of eye movement desensitization and reprocessing and biofeedback/stress inoculation training in treating test anxiety. Journal of EMDR: Practice and Research, 6(2), 62-72. https://doi.org/10.1891/1933-3196.6.2.62

Crawford, M. J., Thana, L., Farquharson, L., Palmer, L., Hancock, E. et al. (2016). Patient experience of negative effects of psychological treatment: Results of a national survey. The British Journal of Psychiatry, 208(3), 260-265. https://doi.org/10.1192/bjp.114.162628

Crews, F. (1995). The memory wars: Freud's legacy in dispute. London, England: Granta Books.

Cuijpers, P., Turner, E. H., Koole, S. L., van Dijke, A., \& Smit, F. (2014). What is the threshold for a clinically relevant effect? The case of major depressive disorders. Depression and Anxiety, 31(5), 374378. https://doi.org/10.1002/da.22249

Cuijpers, P., van Veen, S. C., Sijbrandij, M., Yoder, W., \& Cristea, I. A. (2020). Eye movement desensitization and reprocessing for mental health problems: A systematic review and meta-analysis. Cognitive Behaviour Therapy, 49(3), 165-180. https://doi.org/10.1080/16506073.2019.1703801

*Cuperus, A. A., Laken, M., van Schie, K., Engelhard, I. M., \& van den Hout, M. A. (2019). Dual-tasking during recall of negative memories or during visual perception of images: Effects on vividness and emotionality. Journal of Behavior Therapy and Experimental Psychiatry, 62, 112-116. https://doi.org/10.1016/j.jbtep.2018.10.003

Crombag, H. F. M., \& Merckelbach, H. (1996). Hervonden herinneringen en andere misverstanden [recovered memories and other misconceptions]. Amsterdam: Contact.

Crombag, H.F.M., \& Merckelbach, H.L.G.J. (2019). Hervonden herinneringen en andere misverstanden [recovered memories and other misconceptions]. Amsterdam: Contact.

Cybulski, L., Mayo-Wilson, E., \& Grant, S. (2016). Improving transparency and reproducibility through registration: The status of intervention trial published in clinical psychology journals. Journal of Consulting and Clinical Psychology, 84(9), 753-767. https://doi.org/10.1037/ccp0000115

Dalenberg, C. J., Brand, B. L., Loewenstein, R. J., Frewen, P. A., \& Spiegel, D. (2020). Inviting scientific 


\section{References}

discourse on traumatic dissociation: Progress made and obstacles to further resolution. Psychological Injury and Law, 13, 135-154. https://doi.org/10.1007/s12207-020-09376-9

Dammeyer, M. D., Nunez Nightingale, N. N., \& McCoy, M. L. (1997). Repressed memory and other controversial origins of sexual abuse allegations: Beliefs among psychologists and clinical social workers. Child Maltreatment, 2(3), 252-263. https://doi.org/10.1177/1077559597002003007

Dasse, M. N., Juback, S. K., Morisette, S. B., Dolan, S. L., \& Weaver, C. A. (2015). False memory susceptibility in OEF/ OIF veterans with and without PTSD. Military Psychology, 27(6), 354-365. https://doi.org/10.1037/mil0000094

Davidson, P. R., \& Parker, C. H. (2001). Eye movement desensitization and reprocessing (EMDR): A metaanalysis. Journal of Consulting and Clinical Psychology, 69(2), 305-316. https://doi.org/ 10.1037/0022-006X.69.2.305

De Rivera, J. (1997). The construction of false memory syndrome: The experience of retractors. Psychological Inquiry, 8(4), 271-292. https://doi.org/10.1207/s15327965pli0804_1

De Santis, F. (2007). Alternative Bayes factors: Sample size determination and discriminatory power assessment. Test, 16, 504-522. https://doi.org/10.1007/s11749-006-0017-7

Deferme, D., \& Otgaar, H. (2020). Seksuele misdrijven op minderjarigen: Enkele kritische overwegingen over de onverjaarbaarheid [Sexual offenses against minors: Some critical consideration about the limitation period]. Nieuw Juridisch Weekblad, 424, 474-483.

Deese, J. (1959). On the prediction of occurrence of particular verbal intrusions in immediate recall. Journal of Experimental Psychology, 58(1), 17-22. https://doi.org/10.1037/h0046671

Devilly, G. J., \& Brown, L. (2011). The role of imagery rehearsal with and without eye movements in the creation of false memories. Psychology, Crime, \& Law, 17(6), 529-543. https://doi.org/ 10.1080/10683160903397524.

Devilly, G. J., Ono, M., \& Lohr, J. M. (2014). The use of meta-analytic software to derive hypotheses for EMDR. Journal of Behavior Therapy and Experimental Psychiatry, 45(1), 223-225. https://doi.org/10.1016/j.jbtep.2013.10.004

Dewhurst, S. A., Pursglove, R., \& Lewis, C. (2007). Story contexts increase susceptibility to the DRM illusion in 5-year-olds. Developmental Science, 10(3), 374-378. https://doi.org/10.1111/ j.1467-7687.2007.00592.x

Diehle, J., Opmeer, N. C., Boer, F., Mannarino, A. P., \& Lindauer, R. J. (2015). Trauma-focused cognitive behavioral therapy or eye movement desensitization and reprocessing: What works in children with posttraumatic stress symptoms? A randomized controlled trial. European Child and Adolescent Psychiatry, 24, 227-236. https://doi.org/10.1007/s00787-014-0572-5 
Dodier, O., Melinder, A., Otgaar, H., Payoux, M., \& Magnussen, S. (2019). Psychologists and psychiatrists in court: What do they know about eyewitness memory? A comparison of experts in inquisitorial and adversarial legal systems. Journal of Police and Criminal Psychology, 34, 254262. https://doi.org/10.1007/s11896-019-09339-0

Doering, S., Ohlmeier, M.C., de Jongh, A., Hofmann, A., \& Bisping, V. (2013). Efficacy of a trauma-focused treatment approach for dental phobia: a randomized clinical trial. European Journal of Oral Sciences, 121(6), 584-593. https://doi.org/10.1111/eos.12090

Drivdahl, S. B., \& Zaragoza, M. S. (2001). The role of perceptual elaboration and individual differences in the creation of false memories for suggested events. Applied Cognitive Psychology, 15(3), 265-281. https://doi.org/10.1002/acp.701

Dudukovic, N. M., DuBrow, S., \& Wagner, A. D. (2009). Attention during memory retrieval enhances future remembering. Memory \& Cognition, 37(7), 953-961. https://doi.org/10.3758/MC.37.7.953

Duncan, B. L., Miller, S. D., Sparks, J. A., Claud, D. A., Reynolds, L. R., Brown, J., \& Johnson, L. D. (2003). The session rating scale: Preliminary psychometric properties of a working alliance measure. Journal of Brief Therapy, 3(1), 3-12.

Duval, S., \& Tweedie, R. (2000). Trim and fill: A simple-funnel-plot-based method of testing and adjusting for publication bias in meta-analysis. Biometrics, 56(2), 455-463. https://doi.org/10.1111/ j.0006-341x.2000.00455.x

Dziegielewski, S. F., \& Wolfe, P. (2000). Eye movement desensitization and reprocessing (EMDR) as a time-limited treatment intervention for body image disturbance and self-esteem: A single subject case study design. Journal of Psychotherapy in Independent Practice, 1(3), 1-16. https://doi.org/10.1300/J288v01n03_01

Ehlers, A., \& Clark, D. M. (2000). A cognitive model of posttraumatic stress disorder. Behavior Research and Therapy, 15(3), 249-275. https://doi.org/10.1016/S0005-7967(99)00123-0

Ehring, T., Welboren, R., Morina, N., Wicherts, J. M., Freitag, J., \& Emmelkamp, P. M. G. (2014). Metaanalysis of psychological treatment for posttraumatic stress disorder in adult survivors of childhood abuse. Clinical Psychology Review, 34(8), 645-657. https://doi.org/10.1016/j.cpr.2014.10.004

Eisen, M. L., Gomes, D. M., Lorber, W. G., Perez, G. I., \& Uchishiba, H. (2013). Using an individual differences approach to examine two distinct types of suggestibility effects. Applied Cognitive Psychology, 27(1), 2-11. https://doi.org/10.1002/acp.2864

Eisen, M., Quas, J. A., \& Goodman, G. S. (2002). Memory and suggestibility in the forensic interview. Mahwah, NJ: Lawrence Erlbaum Associates Inc.

Engelhard, I. M., van den Hout, M. A., \& Smeets, M. A. M. (2011). Taxing working memory reduces 


\section{References}

vividness and emotionality of images about the Queen's day tragedy. Journal of Behavior Therapy and Experimental Psychiatry, 42(1), 32-37. https://doi.org/10.1016/j.jbtep.2010.09.004

Engelhard, I. M., van den Hout, M. A., Dek, E. C. P., Giele, C. L., van der Wielen, J.-W., Reijnen, M. J., \& van Roij, B. (2011). Reducing vividness and emotional intensity of recurrent "flashforwards" by taxing working memory: An analogue study. Journal of Anxiety Disorders, 25(4), 599-603. https://doi.org/10.1016/j.janxdis.2011.01.009

Engelhard, I. M., van den Hout, M. A., Janssen, W. C., \& van der Beek, J. (2010). Eye movements reduce vividness and emotionality of "flashforwards". Behaviour Research and Therapy, 48(5), 442447. https://doi.org/10.1016/j.brat.2010.01.003

*Engelhard, I. M., Sijbrandij, M., van den Hout, M. A., Rutherford, N. M., Rahim, H. F., \& Kocak, F. (2012). Choking under pressure: Degrading flashforwards related to performance anxiety. Journal of Experimental Psychopathology, 3, 158-167. https://doi.org/10.5127/jep.024111

*Engelhard, I. M., van Uijen, S. L., \& van den Hout, M. A. (2010). The impact of taxing working memory on negative and positive memories. European Journal of Psychotraumatology, 1, 1-8. https://doi.org/10.3402/ejpt.v1i0.5623

Erdelyi, M. H. (2006). The unified theory of repression. Behavioural and Brain Sciences, 29(5), 499-551. https://doi.org/10.1017/S0140525X06009113

Estergard, L. (2009). Eye Movement Desensitization and Reprocessing in the Treatment of Chronic Pain. Walden: Walden University.

Faul, F., Erdfelder, E., Lang, A.-G., \& Buchner, A. (2007). G*Power 3: A flexible statistical power analysis program for the social, behavioural, and biomedical sciences. Behavior Research Methods, 39(2), 175-191. https://doi.org/10.3758/BF03193146.

Fernandes, M. A., \& Moscovitch, M. (2000). Divided attention and memory: Evidence of substantial interference effects at retrieval and encoding. Journal of Experimental Psychology: General, 129(2), 155-176. https://doi.org/10.1037//0096-3445.129.2.155

Feske, U., \& Goldstein, A. J. (1997). Eye Movement Desensitization and Reprocessing treatment for panic disorder: a controlled outcome and partial dismantling study. Journal of Consultancy Clinical Psychology, 65(6), 1026-1035. https://doi.org/10.1037/0022-006X.65.6.1026

Field, A., \& Cottrell, D. (2011). Eye movement desensitization and reprocessing as a therapeutic intervention for traumatized children and adolescents: A systematic review of the evidence for family therapists. Journal of Family Therapy, 33(4), 374-388. https://doi.org/10.1111/ j.1467-6427.2011.00548.x

Finnie, P. S. B., \& Nader, K. (2012). The role of metaplasticity mechanisms in regulating memory 
destabilization and reconsolidation. Neuroscience \& Biobehavioral Reviews, 36(7), 16671707. https://doi.org/10.1016/j.neubiorev.2012.03.008

Fivush, R. (1997). Event memory. In N. Cowan (Ed.), The development of memory in childhood (pp. 139162). Hove: Psychology Press.

Fivush, R., \& Edwards, V. J. (2004). Remembering and forgetting childhood sexual abuse. Clinical and Qualitative Research, 13(2), 1-19. https://doi.org/10.1300/J070v13n02_01

Fjermestad, K. W., Lerner, M. D., McLeod, B. D., Wergeland, G. J. H., Haugland, B. S. M., Havik, O. E., Öst, L.-G., \& Silverman, W. K. (2017). Motivation and treatment credibility predict alliance in cognitive behavioral treatment for youth with anxiety disorders in community clinics. Journal of Clinical Psychology, 74(6), 793-805. https://doi.org/10.1002/jclp.22551

Foa, E. B., \& Riggs, D. S. (1993). Post-traumatic stress disorder in rape victims. In J. Oldham, M. B. Riba, \& A. Tasman (Eds.), American Psychiatric Press Review of Psychiatry, vol. 12 (pp. 273-303). Washington, DC: American Psychiatric Press.

Foa, E. B., \& Rothbaum, B. O. (1998). Treating the trauma of rape: cognitive behavioral therapy for PTSD. New York: Guilford Press.

Foa, E. B., Steketee, G., \& Rothbaum, B. O. (1989). Behavioral/cognitive conceptualisation of posttraumatic stress disorder. Behavior Therapy, 20(2), 155-176. https://doi.org/10.1016/S00057894(89)80067-X

Foley, T., \& Spates, R. (1995). Eye movement desensitization of public-speaking anxiety: A partial dismantling. Journal of Behavior Therapy and Experimental Psychiatry, 26(4), 321-329. https://doi/org/10.1016/0005-7916(95)00048-8

Frenda, S. J., Nichols, R. M., \& Loftus, E. F. (2011). Current issues and advances in misinformation research. Current Directions in Psychological Science, 20(1), 20-23. https://doi.org/10.1177/ 0963721410396620

Freud, S. (1910). The origin and development of psychoanalysis. The American Journal of Psychology, 21, 181-218. https://doi.org/10.1037/11350-001

Freud, S. (1954). The aetiology of hysteria. In J. Strachey (Ed.), The complete psychological works of Sigmund Freud, standard edition. New York, NY: Free Press.

Freyd, J. J. (1994). Betrayal-trauma: Traumatic amnesia as an adaptive response to childhood abuse. Ethics \& Behavior, 4(4), 307-329. https://doi.org/10.1207/s15327019eb0404_1

Friedberg, F. (2004). Eye movement desensitization in fibromyalgia: A pilot study. Complementary Therapies in Nursing and Midwifery, 10(4), 245-249. https://doi.org/10.1016/j.ctnm.2004.06.006 Friedman, M. J. (1996). PTSD diagnosis and treatment for mental health clinicians. Community Mental 


\section{References}

Health Journal, 32(2), 173-189. https://doi.org/10.1007/BF02249755

Gallo, D. A. (2006). Associative illusion of memory: False memory research in DRM and related tasks. New York: Psychology Press.

Gallo, D. A. (2010). False memories and fantastic beliefs: 15 years of the DRM illusion. Memory \& Cognition, 38, 833-848. https://doi.org/10.3758/MC.38.7.833

Gallo, D. A., \& Roediger, H. L. (2002). Variability among word lists in eliciting memory illusions: Evidence for associative activation and monitoring. Journal of Memory and Language, 47(3), 469-497. https://doi.org/10.1016/S0749-596X(02)00013-X

Gallo, D. A., Bell, D. M., Beier, J. S., \& Schacter, D. L. (2006). Two types of recollection-based monitoring in younger and older adults: Recall-to-reject and the distinctiveness heuristic. Memory, 14(6), 730-741. https://doi.org/10.1080/09658210600648506

Gerhardt, A. (2016). Eye Movement Desensitization and reprocessing vs. treatment-as-usual for nonspecific chronic back pain patients with psychological trauma: A randomized controlled pilot study. Frontiers in Psychiatry, 7:201. https://doi.org/10.3389/fpsyt.2016.00201

Gerrie, M. P., \& Garry, M. (2011). Warnings reduce false memories for missing aspects of events. Experimental Psychology, 58(3), 207-216. https://doi.org/10.1027/1618-3169/a000087

Gielkes, E. M. J., Sobczak, S., \& Van Alphen, S. P. J. (2016). Eye movement desensitization and reprocessing therapy for personality disorders in older adults? International Psychogeriatrics, 28(10), 1751-1752. https://doi.org/10.1017/S1041610216000892

Goff, L. M., \& Roediger, H. L. (1998). Imagination inflation for action events: Repeated imaginings lead to illusory recollections. Memory \& Cognition, 26, 20-33. https://doi:10.3758/ BF03211367

Gold, J., \& Stricker, G. (2011). Failures in psychodynamic psychotherapy. Journal of Clinical Psychology: In Session, 67(11), 1096-1105. https://doi.org/10.1002/jclp.20847

Goldfarb, D., Goodman, G. S., Larson, R. P., Eisen, M. L., \& Qin, J. (2019). Long-term memory in adults exposed to childhood violence: Remembering genital contact nearly 20 years later. Clinical Psychological Science, 7(2), 381-396. https://doi.org/1177/2167702618805742

Goldstein, A. J., de Beurs, E., Chambless, D. L., \& Wilson, K. A. (2000). EMDR for panic disorder with agoraphobia: comparison with waiting list and credible attention-placebo control condition. Journal of Consultancy and Clinical Psychology, 68(6), 947-956. https://doi.org/10.1037/ 0022-006X.68.6.947

Goodman, S. N. (1999). Toward evidence-based medical statistics 2: The Bayes factor. Annals of Internal Medicine, 130(12), 1005-1013. https://doi.org/10.7326/0003-4819-130-12-199906150-00019

Goodman, G. S., Ghetti, S., Quas, J. A., Edelstein, R. S., Weede Alexander, K., Redlich, A. D., Cordon, I. M., 
\& Jones, D. P. H. (2003). A prospective study of memory for child sexual abuse: New findings relevant to the repressed-memory controversy. Psychological Science, 14(2), 113-118. https://doi.org/10.1111/1467-9280.01428

Goodman, G. S., Ogle, C. M., Block, S. D., Larson, R. P. Augusti, E.-M., Cho, Y. I., Beber, J., Timmer, S., \& Urquiza, A. (2011). False memory for trauma-related DRM lists in adolescents and adults with histories of child sexual abuse. Developmental Psychology, 23(2), 423-438. https://doi.org/ $10.1017 /$ S0954579411000150

Golding, J. M., Sanchez, R. P., \& Sego, S. A. (1996). Do you believe in repressed memories? Professional Psychology: Research and Practice, 27(5), 429-437. https://doi.org/10.1037/0735-7028.27.5.429

Gore-Felton, C., Koopman, C., Thoresen, C., Arnow, B., Bridges, E., \& Spiegel, D. (2000). Psychologists' beliefs and clinical characteristics: Judging the veracity of childhood sexual abuse memories. Professional Psychology: Research and Practice, 31(4), 372-377. https://doi.org/10.1037/ 0735-7028.31.4.372

Gosselin, P., \& Matthews, W. J. (1995). Eye movement desensitization and reprocessing in the treatment of test anxiety: A study of the effects of expectancy and eye movement. Journal of Behavior Therapy and Experimental Psychiatry, 26(4), 331-337. https://doi.org/10.1016/0005-7916 (95)00038-0

Grant, M. (2000). EMDR: A new treatment for trauma and chronic pain. Complementary Therapies in Nursing and Midwifery, 6(2), 91-94. https://doi.org/10.1054/ctnm.2000.0459

Grant, M., \& Threlfo, C. (2002). EMDR in the treatment of chronic pain. Journal of Clinical Psychology, 58(12), 1505-1520. https://doi/org/10.1002/jclp.10101

Grasman, R. (2017). Meta-analysis in JASP. Web publication/site, JASP. Retrieved from https://jasp-stats. org/2017/1/15/meta-analysis-jasp

Greenwald, R., McClintock, S. D., \& Bailey, T. D. (2013). A controlled comparison of eye movements desensitization and reprocessing and progressive counting. Journal of Aggression, Maltreatment, \& Trauma, 22(9), 981-996. https://doi.org/10.1080/10926771.2013.834020

Greenwald, R., McClintock, S. D., Jarecki, K., \& Monaco, A. J. (2015). A comparison of eye movement desensitization and reprocessing and progressive counting among therapists in training. Traumatology, 21(1), 1-6. https://doi.org/10.1037/trm0000011

Gunter. R. W., \& Bodner, G. E. (2008). How eye movements affect unpleasant memories: Support for a working-memory account. Behaviour Research and Therapy, 46(8), 913-931. https://doi.org/ 10.1016/j.brat.2008.04.006

Gunter, R. W., \& Bodner, G. E. (2009). EMDR works... but how? Recent progress in the search for treatment 


\section{References}

mechanisms. Journal of EMDR Practice and Research, 3(3), 161-168. https://doi.org/10.1891/ 1933-3196.3.3.161

Gupta, M. A., \& Gupta, A. K. (2002). Use of eye movement desensitization and reprocessing (EMDR) in the treatment of dermatologic disorders. Journal of Cutaneous Medicine and Surgery: Incorporating Medical and Surgical Dermatology, 6(5), 415-421. https://doi.org/10.1107/ s10227-0016-8

Hafkenscheid, A. (2014). De therapeutische relatie [The therapeutic relationship]. Enschede: De Tijdstroom.

Halligan, S. L., Michael, T., Clark, D. M., \& Ehlers, A. (2003). Posttraumatic stress disorder following assault: The role of cognitive processing, trauma memory, and appraisals. Journal of Consulting and Clinical Psychology, 71(3), 419-431. https://doi.org/10.1037/0022-006X.71.3.419

Halvgaard, K. (2015). Single Case Study: Does EMDR Psychotherapy Work on Emotional Eating? Journal of EMDR Practice and Research, 9(4), 188-197. https://doi.org/10.1891/1933-3196.9.4.188

Hannan, C., Lambert, M.J., Harmon, C., Nielsen, S.L., Smart, D.W. et al. (2005). A lab test and algorithms for identifying clients at risk for treatment failure. Journal of Clinical Psychology, 61(2), 155-163. https://doi.org/10.1002/jclp.20108

Hansen, N. B., Lambert, M. J., \& Forman, E. M. (2002). The psychotherapy dose-response effect and its implications for treatment delivery services. Clinical Psychology: Science and Practice, 9(3), 329-343. https://doi.org/10.1093/clpsy.9.3.329

Hase, M., Balmaceda, U. M., Hase, A., Lehnung, M., Tumani, V., Huchzermeier, C., \& Hofmann, A (2015). Eye movement desensitization and reprocessing (EMDR) therapy in the treatment of depression: A matched pairs study in an inpatient setting. Brain and Behavior, 5(6), 1-9. https://doi.org/10.1002/brb3.342

Hase, M., Schallmayer, S., \& Sack, M. (2008). EMDR Reprocessing of the addiction memory: pretreatment, posttreatment, and 1-month follow-up. Journal of EMDR Practice and Research, 2(3), 170-179. https://doi.org/10.1891/1933-3196.2.3.170

Hassard, A. (1995). Investigation of eye movement desensitization in pain clinic patients. Behavioural and Cognitive Psychotherapy, 23(2), 177-185. https://doi.org/10.1017/S1352465800014429

Hatfield, D., McCullough, L., Frantz, S.H., \& Krieger, K. (2010). Do we know when our clients get worse? An investigation of therapists' ability to detect negative client change. Clinical Psychology \& Psychotherapy, 17(1), 25-32. https://doi.org/10.1002/cpp.656

Henkel, L. A., Franklin, N., \& Johnson, M. K. (2000). Cross-modal source monitoring confusions between perceived and imagined events. Journal of Experimental Psychology: Learning, Memory, and Cognition, 26(2), 321-335. https://doi.org/10.1037/0278-7393.26.2.321 
Herbert, J. D., Lilienfeld, S. O., Lohr, J. M., Montgomery, R. W., O'Donohue, W. T., Rosen, G. M., \& Tolin, D. F. (2000). Science and pseudoscience in the development of eye movement desensitization and reprocessing: Implications for clinical psychology. Clinical Psychology Review, 20(8), 945971. https://doi.org/10.1016/S0272-7358(99)00017-3

Higgins, J. P. T., \& Thompson, S. G. (2002). Quantifying heterogeneity in a meta-analysis. Statistics in Medicine, 21(11), 1539-1558. https://doi.org/10.1002/sim.1186

Higham, P. A. (1998). Believing details known to have been suggested. British Journal of Psychology, 89 (2), 265-283. https://doi.org/10.1111/j.2044-8295.1998.tb02684.x

von Hippel, P. T. (2015). The heterogeneity statistic 12 can be biased in small meta-analysis. BMC Medical Research Methodology, 15:35. https://doi.org/10.1186/s12874-015-0024-z

Hofmann, A., Hilgers, A., Lehnung, M., Liebermann, P., Ostacoli, L., Schneider, W., \& Hase, M. (2014). Eye movement desensitization and reprocessing as an adjunctive treatment of unipolar depression: A controlled study. Journal of EMDR Practice and Research, 8(3), 103-112. https://doi.org/10.1891/1933-3196.8.3.103

Hogan, W. A. (2001). The comparative effects of eye movement desensitization and reprocessing (EMDR) and cognitive behavioral therapy (CBT) in the treatment of depression. ProQuest Information \& Learning: U.S.

Holland, A. C., \& Kensinger, E. A. (2010). Emotion and autobiographical memory. Physics of Life Reviews, 7(1), 88-131. https://doi.org/10.1016/j.plrev.2020.01.006

Holmes, E. A., \& Bourne, C. (2008). Inducing and modulating intrusive emotional memories: A review of the trauma film paradigm. Acta Psychologica, 127(3), 553-566. https://doi.org/10.1016/ j.actpsy.2007.11.002

Holmes, E.A., Ghaderi, A., Harmer, C. J., Ramchandani, P.G., Cuijpers, P. et al. (2018). The Lancet Psychiatry commission on psychological treatments research in tomorrow's science. The Lancet Psychiatry, 5(3), 237-286. https://doi.org/10.1016/\$2215-0366(17)30513-8

Hornstein, G. A. (1992). The return of the repressed: Psychology's problematic relations with psychoanalysis, 1909-1960. American Psychologist, 47(2), 254-263 https://doi.org/10.1037//0003-066X.47.2.254.

Hornsveld, H., ten Broeke, E., \& de Jongh, A. (2017a). Verschillen tussen het Nederlandse EMDRstandaardprotocol en het originele protocol van Shapiro. Deel 1: De NC [Differences between the Dutch EMDR standard protocol and the original protocol of Shapiro. Part 1: The NC]. EMDR magazine, 5(13), 42-45.

Hornsveld, H., ten Broeke, E., \& de Jongh, A. (2017b). Verschillen tussen het Nederlandse EMDRstandaardprotocol en het originele protocol van Shapiro. Deel 2: De casusconceptualisatie 


\section{References}

[Differences between the Dutch EMDR standard protocol and the original protocol of Shapiro. Part 2: Case conceptualization]. EMDR magazine, 1(14), 44-47.

Hornsveld, H., ten Broeke, E., \& de Jongh, A. (2017c). Verschillen tussen het Nederlandse EMDRstandaardprotocol en het originele protocol van Shapiro. Deel 3: Machteloos makende targets [Differences between the Dutch EMDR standard protocol and the original protocol of Shapiro. Part 3: Powerless targets]. EMDR magazine, 5(15), 44-48.

Hornsveld, H., ten Broeke, E., \& de Jongh, A. (2018a). Verschillen tussen het Nederlandse EMDRstandaardprotocol en het originele protocol van Shapiro. Deel 4: Scherpstellen, desensitisatie en 'back to target' [Differences between the Dutch EMDR standard protocol and the original protocol of Shapiro. Part 4: Focusing, desensitization and back to target]. EMDR magazine, 6 (16), 33-36.

Hornsveld, H., ten Broeke, E., \& de Jongh, A. (2018b). Verschillen tussen het Nederlandse EMDRstandaardprotocol en het originele protocol van Shapiro. Deel 5: De werkgeheugentheorie [Differences between the Dutch EMDR standard protocol and the original protocol of Shapiro. Part 5: The working memory account]. EMDR magazine, 6(18), 39-43.

Hornsveld, H. K., Houtveen, J., Vroomen, M., Aalbers, I., Kapteijn, I., Aalbers, D., \& van den Hout, M. A. (2011). Evaluating the effect of eye movements on positive memories such as those used in resource development and installation. Journal of EMDR Practice and Research, 5(4), 146155. https://doi.org/10.1891/1933-3196.5.4.146

Hornsveld, H. K., Landwehr, F., Stomp, M. P. H., Smeets, M. A. M., \& van den Hout, M. A. (2010). Emotionality of loss-related memories is reduced after recall plus eye movements but not after recall plus music or recall only. Journal of EMDR Practice and Research, 4(3), 106-112. https://doi.org/10.1891/1933-3196.4.3.106

Horst, F., Den Oudsten, B., Zijlstra, W., de Jongh, A., Lobbestael, J., \& De Vries, J. (2017). Cognitive behavioral therapy vs. Eye movement desensitization and reprocessing for treating panic disorder: a randomized controlled trial. Frontiers in Psychology, 8(1409). https://doi.org/ 10.3389/fpsyg.2017.01409

Houben, S. T. L. (2020). Tinder voor psychotherapie? Over de blinde vlek van therapeuten. [Tinder for psychotherapy? About the blind spot of therapists]. De Psycholoog, 55, 10-18.

Houben, S. T. L., Otgaar, H., Roelofs, J., \& Merckelbach, H. (2018). Lateral eye movements increase false memory rates. Clinical Psychological Science, 6(4), 610-616. https://doi.org/10.1177/ 2167702618757658

Houben, S. T. L., Otgaar, H., Roelofs, J., \& Merckelbach, H. (2019). EMDR and false memories: A response 
to Lee, de Jongh, and Hase (2019). Clinical Psychological Science, 7(3), 405-406.

https://doi.org/10.1177/2167702619830392

Houben, S. T. L., Otgaar, H., Roelofs, J., Smeets, T., \& Merckelbach, H. (2020). Increases of correct memories and spontaneous false memories due to eye movements when memories are retrieved after a time delay. Behavior Research and Therapy, 125, 103546. https://doi.org/ 10.1016/j.brat.2019.103536

Houben, S. T. L., Otgaar, H., Roelofs, J., Wessel, I., Patihis, L., \& Merckelbach, H. (2019). Eye Movement Desensitization and Reprocessing (EMDR) practitioners' beliefs about memory. Psychology of Consciousness: Theory, Research and Practice. https://doi.org/10.1037/cns0000211 van den Hout, M. A., \& Engelhard, I. M. (2012). How does EMDR work? Journal of Experimental Psychology, 3(5), 724-738. https://doi.org/10.5127/jep.028212

van den Hout, M. A., Bartelski, N., \& Engelhard, I. M. (2013). On EMDR: Eye movements retrieval reduce subjective vividness and objective memory accessibility during future recall. Cognition and Emotion, 27(1), 177-183. https://doi.org/10.1080/02699931.2012.691087

van den Hout, M. A., Eidhof, M. B., Verboom, J., Littel, M., \& Engelhard, I. M. (2014). Blurring of emotional and non-emotional memories by taxing working memory during recall. Cognition \& Emotion, 28(4), 717-727. https://doi.org/10.1080/02699931.2013.848785

van den Hout, M. A., Engelhard, I. M., Beetsma, D., Slofstra, C., Hornsveld, H., Houtveen, J., \& Leer, A. (2011). EMDR and mindfulness. Eye movements and attentional breathing tax working memory and reduce vividness and emotionality of aversive ideation. Journal of Behavior Therapy and Experimental Psychiatry, 42(4), 423-431. https://doi.org/10.1016/j.jbtep.2011.03.004

*van den Hout, M. A., Engelhard, I. M., Rijkeboer, M., Koekebakker, J., Hornsveld, H., Leer, A., et al. (2011). EMDR: Eye movements superior to beeps in taxing working memory and reducing vividness of recollections. Behaviour Research and Therapy, 49(2), 92-98. https://doi.org/ 10.1016/j.brat.2010.11.003

*van den Hout, M. A., Engelhard, I. M., Smeets, M. A. M., Hornsveld, H., Hoogeveen, E., de Heer, E., et al. (2010). Counting during recall: taxing of working memory and reduced vividness and emotionality of negative memories. Applied Cognitive Psychology, 24(3), 303-311. https://doi.org/10.1002/acp.1677

*van den Hout, M., Muris, P., Salemink, E., \& Kindt, M. (2001). Autobiographical memories become less vivid and emotional after eye movements. British Journal of Clinical Psychology, 40(2), 121130. https://doi.org/10.1348/014466501163571

van den Hout, M. A., Rijkeboer, M. M., Engelhard, I. M., Klugkist, I., Hornsveld, H., Toffolo, M. J. B., et al. 


\section{References}

(2012). Tones inferior to eye movements in the EMDR treatment of PTSD. Behaviour Research and Therapy, 50(5), 275-279. https://doi.org/10.1016/j.brat.2012.02.001

Hovland, R. T., Ytrehus, S., Mellor-Clark, J., \& Moltu, C. (2020). How patients and clinicians experience the utility of a personalized clinical feedback system. Journal of Clinical Psychology, 1-18. https://doi.org/10.1002/jclp.22992

Howe, M. L., \& Knott, L. M. (2015). The fallibility of memory in judicial processes: Lessons from the past and their modern consequences. Memory, 23(5), 633-656. https://doi.org/10.1080/0965821 1.2015.1010709

Howe, M. L., Candel, I., Otgaar, H., Malone, C., \& Wimmer, M. C. (2010). Valence and the development of immediate and long-term false memory illusions. Memory, 18(1), 58-75. https://doi.org/ $10.1080 / 09658210903476514$

Howe, M. L., Wimmer, M. C., Gagnon, N., \& Plumpton, S. (2009). An associative-activation theory of children's and adults' memory illusions. Journal of Memory and Language, 60(2), 229-251. https://doi.org/10.1016/j.jml.2008.10.002

Hudson, J. I., Chase, E. A., \& Pope, H. G. jr. (1998). Eye movement desensitization and reprocessing in eating disorders: Caution against premature acceptance. International Journal of Eating Disorders, 23(1), 1-5. https://doi.org/10.1002./(SICI)1098-108C)199801)

Hyman, I. E., \& Billings, F. J. (1998). Individual differences and the creation of false childhood memories. Memory, 6(1), 1-20. https://doi.org/10.1080/741941598

Hyman, I. E., Husband, T. H., \& Billings, F. J. (1995). False memories of childhood experiences. Applied Cognitive Psychology, 9(3), 181-197. https://doi.org/10.1002/acp.2350090302

Innocence Project (2020). Retrieved from https://www.innocenceproject.org

Janse, P. D., de Jong, K., van Dijk, M. K., Hutschemaekers, G. J., \& Verbraak, M. J. (2017). Improving the efficiency of cognitive-behavioural therapy by using formal client feedback. Psychotherapy Research, 27(5), 525-538. https://doi.org/10.1080/10503307.2016.1552408

Jaschinski, U., \& Wentura, D. (2002). Misleading postevent information and working memory capacity: An individual differences approach to eyewitness memory. Applied Cognitive Psychology, 16 (2), 223-231. https://doi.org/10.1002/acp.783

JASP Team (2019). JASP (Version 0.11.1) [Computer software]. Retrieved from http://jasp-stats.org John-Baptiste Bastien, R., Jongsma, H., KAbadayi, M., \& Billings, J. (2020). The effectiveness of psychological interventions for post-traumatic stress disorder in children, adolescents and young adults: A systematic review and meta-analysis. Psychological Medicine, 50, 1598-1612. https://doi.org/ 10.1017/S0033291720002007 
Johnson, M. K. (1988). Reality monitoring: An experimental phenomenological approach. Journal of Experimental Psychology: General, 117(4), 390-394. https://doi.org/10.1037/0096-3445.117.4.390

Johnson, M. K. (1991). Reality monitoring: Evidence from confabulation in organic brain disease patients. In G. P. Prigatano \& D. L. Schacter (Eds.), Awareness of deficit after brain injury: Clinical and theoretical issues (pp. 176-197). New York: Oxford University Press.

Johnson, M. K., \& Raye, C. L. (1981). Reality monitoring. Psychological Review, 88(1), 67-85. https://doi.org/10.1037/0033-295X.88.1.67

Johnson, M. K., Foley, M. A., \& Leach, K. (1988). The consequences for memory of imagining in another person's voice. Memory \& Cognition, 16, 337-342. https://doi.org/10.3758/BF03197044

Johnson, M. K., Hashtroudi, S., \& Lindsay, D. S. (1993). Source monitoring. Psychological Bulletin, 114(1), 3-28. https://doi.org/ 10.1037/0033-2909.114.1.3

Johnson, M. K., Kahan, T. L., \& Raye, C. L. (1984). Dreams and reality monitoring. Journal of Experimental Psychology: General, 113(3), 329-344. https://doi.org/10.1037/0096-3445.113.3.329

Johnson, M. K., Raye, C. L., Wang, A. Y., \& Taylor, T. H. (1979). Fact and fantasy: The roles of accuracy and variability in confusing imaginations with perceptual experiences. Journal of Experimental Psychology: Human Learning and Memory, 5(3), 229-240. https://doi.org/10.1037/ 0278-7393.5.3.229

Jones, J. C., \& Barlow, D. H. (1990). The etiology of posttraumatic stress disorder. Clinical Psychology Review, 10(3), 299-328. https://doi.org/10.1016/0272-7358(90)90064-H

de Jongh, A., \& Bicanic, I. (2020). Kan EMDR leiden tot pseudoherinneringen? [Can EMDR lead to false memories?]. Retrieved from: https://www.emdr.nl/kan-emdr-leiden-tot-pseudo-herinneringen de Jongh, A., \& ten Broeke, E. (2016). Handboek EMDR: Een geprotocolleerde behandelmethode voor de gevolgen van psychotrauma [EMDR handbook: A treatment protocol for the consequences of psychotrauma]. Amsterdam, the Netherlands: Pearson.

de Jongh, A., \& Wessel, I. (2018). Geen herinnering? Niet behandelen! [No memory? Do not treat!]. EMDR Magazine, 17, 58-59.

de Jongh, A., Ernst, R., Marques, L., \& Hornsveld, H. (2013). The impact of eye movements and tones on disturbing memories involving PTSD and other mental disorders. Journal of Behavior Therapy and Experimental Psychiatry, 44, 477-483. https://doi.org/10.1016/j.jbtep.2013.07.002

Jonsson, U., Alaie, I., Parling, T., \& Arnberg, F. K. (2014). Reporting of harms in randomized controlled trials of psychological interventions for mental and behavioral disorders: A review of current practice. Contemporary Clinical Trials, 38(1), 1-8. https://doi.org/10.1016/j.cct.2014.02.005 Kahneman, D., \& Klein, G. (2009). Conditions for intuitive expertise: A failure to disagree. American 


\section{References}

Psychologist, 64(6), 515-526. https://doi.org/10.1037/n0016755

Kanter, J. W., Kohlenberg, R. J., \& Loftus, E. F. (2002). Demand characteristics, treatment rationales, and cognitive therapy for depression. Prevention \& Treatment, 5(1), Article 41. https://doi.org/ 10.1037.1522-3736.5.1.541c

Kaplan, R. L., Van Damme, I., \& Levine, L. J. (2012). Motivation matters: Differing effects of pre-goal and post-goal emotions on attention and memory. Frontiers in Psychology, 3:404. https://doi.org/ 10.3389/fpsyg.2012.00404

Kaplan, R. L., Van Damme, I., Levine, L. J., \& Loftus, E. F. (2016). Emotion and false memory. Emotion Review, 8(1), 8-13. https://doi.org/10.1177/1754073915601228

Kavakcı, O., Semiz, M., Kaptanoglu, E., \& Ozer, Z. (2012). EMDR treatment in fibromyalgia, a study of seven cases. Anatolian Journal of Psychiatry, 13(1), 75-81.

Kavanagh, D. J., Freese, S., Andrade, J., \& May, J. (2001). Effects of visuospatial tasks on desensitization to emotive memories. British Journal of Clinical Psychology, 40(3), 267-280. https://doi.org/ 10.1348/014466501163689

Kearns, M., \& Engelhard, I. M. (2015). Psychophysiological responsivity to script-driven imagery: An exploratory study of the effects of eye movements on public speaking flashforwards. Frontiers in Psychiatry, 6, 115. https://doi.org/10.3389/fpsyt.2015.00115

Kelley, S. D., \& Benbadis, S. (2007). Eye movement desensitization and reprocessing in the psychological treatment of trauma-based psychogenic non-epileptic seizures. Clinical Psychology and Psychotherapy, 14(2), 135-144. https://doi.org/10.1002/cpp.525

*Kemps, E., \& Tiggemann, M. (2007). Reducing the vividness and emotional impact of distressing autobiographical memories: The importance of modality-specific interference. Memory, 15 (4), 412-422. https://doi.org/10.1080/09658210701262017

Kensinger, E. A., \& Schacter, D. L. (1999). When true memories suppress false memories: Effects of aging. Cognitive Neuropsychology, 16(3-5), 399-415. https://doi.org/10.1080/026432999380852

Khan, A. M., Dar, S., Ahmed, R., Bachu, R., Adnan, M., \& Padma Kotapati, V. (2018). Cognitive behavioural therapy versus eye movement desensitization and reprocessing in patients with posttraumatic stress disorder: Systematic review and meta-analysis of randomized clinical trials. Cureuc, 10(9), e3250. https://doi.org/10.7759/cureus.3250

Kim, D., Choi, J., Kim, S. H., Oh, D. H., Park, S., \& Lee, S. H. (2010). A pilot study of brief eye movement desensitization and reprocessing (EMDR) for treatment of acute phase schizophrenia. Korean Journal of Biological Psychiatry, 17(2), 94-102.

Knott, L. M., \& Dewhurst, S. A. (2007a). The effects of divided attention at study and test on false 
recognition: A comparison of DRM and categorized lists. Memory \& Cognition, 35, 19541965. https://doi.org/10.3758/BF03192928

Knott, L. M., \& Dewhurst, S. D. (2007b). Divided attention at retrieval disrupts knowing but not remembering. Memory, 15(6), 664-674. https://doi.org/10.1080/09658210701467137

van der Kolk, B. A., \& Fisler, R. (1995). Dissociation and the fragmentary nature of traumatic memories: Overview and exploratory study. Journal of Traumatic Stress, 8(4), 505-525. https://doi.org/ 10.1007/BF02102887

Konuk, E., Epözdemir, H., Atçeken, S. H., Aydin, Y. E., \& Yurtsever, A. (2011). EMDR treatment of migraine. Journal of EMDR: Practice \& Research, 5(4), 166-176. https://doi.org/10.1891/1933-3196.5.4.166

Koss, M. P., Figueredo, A. J., Bell, I., Tharan, M., \& Tromp, S. (1996). Traumatic memory characteristics: a cross-validated mediational model of response to rape among employed women. Journal of Abnormal Psychology, 105(3), 421-432. https://doi.org/10.1037/0021-843X.105.3.421

*Kristjánsdóttir, K., \& Lee, W. C. (2011). A comparison of visual versus auditory concurrent tasks on reducing distress and vividness of aversive autobiographical memories. Journal of EMDR Practice and Research, 5(2), 34-41. https://doi.org/10.1891/1933-3196.5.2.34

Kredlow, M. A., de Voogd, L., \& Phelps, E. A. (2020). Laboratory analogues and therapy procedures: A case for translation from the clinic to the laboratory. PsyArXiv Preprints. https://doi.org/ 10.31234/osf.io/7r6p2

LaBar, K. S., \& Phelps, E. A. (1998). Arousal-mediated memory consolidation: Role of the medial temporal lobe in humans. Psychological Science, 9(6), 490-493. https://doi.org/10.1111/1467-9280.00090

Lakens, D., Scheel, A. M., \& Isager, P. M. (2018). Equivalence testing for psychological research: A tutorial. Advances in Methods and Practices in Psychological Science, 1(2), 259-269. https://doi.org/ $10.1177 / 2515245918770963$

Lambert, M. J., Harmon, C., Slade, K., Whipple, J. L., \& Hawkins, E. J. (2005). Providing feedback to psychotherapists on their patients' progress: Clinical results and practice suggestions. Journal of Clinical Psychology, 61(2), 165-174. https://doi.org/10.1002/jclp.20113

Lambert, M. J., Morton, J. J., Hatfield, D., Harmon, C., Hamilton, S. et al. (2004). Administration and scoring manual for the Outcome Questionnaire-45. Salt Lake City, UT: OQ Measures.

Laney, C., \& Loftus, E. F. (2008). Emotional content of true and false memories. Memory, 16(5), 500-516. https://doi.org/10.1080/09658210802065939

Landing-Romero, R., Moreno-Alcazar, A., Pagani, M., \& Amann, B. L. (2018). How does eye movements desensitization and reprocessing therapy work? A systematic review on suggested mechanisms of action. Frontiers in Psychology, 9:1395. https://doi.org/10.3389/fpsyg.2018.01395 


\section{References}

Leander, L., Christianson, S. Å., \& Granhag, P. A. (2007). A sexual abuse case study: Children's memories and reports. Psychiatry, Psychology and Law, 14(1), 120-129. https://doi.org/10.1375/pplt.14.1.120

Leding, J. K., \& Antonio, L. (2019). Need for cognition and discrepancy detection in the misinformation effect. Journal of Cognitive Psychology, 31(4), 409-415. https://doi.org/10.1080/20445911. 2019.1626400

Lee, C. W., \& Cuijpers, P. (2013). A meta-analysis of the contribution of eye movements in processing emotional memories. Journal of Behavior Therapy and Experimental Psychiatry, 44(2), 231239. https://doi.org/10.1016/j.jbtep.2012.11.001

Lee, C. W., de Jongh, A., \& Hase, M. (2019). Lateral eye movements, EMDR, and memory changes: A critical commentary on Houben et al. (2018). Clinical Psychological Science, 7(3), 403-404. https://doi.org/10.1177/2167702619830395

Leer, A., \& Engelhard, I. M. (2020). Side effects of induced lateral eye movements during aversive ideation. Journal of Behavior Therapy and Experimental Psychiatry, 68: 101566. https://doi.org/10.1016/ j.jbteo.2020.101566

*Leer, A., Engelhard, I. M., \& van den Hout, M. (2014). How eye movements in EMDR work: Changes in memory vividness and emotionality. Journal of Behavior Therapy and Experimental Psychiatry, 45(3), 396-401. https://doi.org/10.1016/j.jbtep.2014.04.004

Leer, A., Engelhard, I. M., Dibbets, P., \& van den Hout, M. A. (2013). Dual-tasking attenuates the return of fear after extinction. Journal of Experimental Psychopathology, 325-340. https://doi.org/ 10.5127/jep.029412

Leer, A., Engelhard, I. M., Lenaert, B., Struyf, D., Vervliet, B., \& Hermans, D. (2017). Eye movement during recall reduces objective memory performance: An extended replication. Behaviour Research and Therapy, 92, 94-105. https://doi.org/10.1016/j.brat.2017.03.002

Lei, S., \& Zhen-Ying, W. (2007). Sertraline treatment of depression combined EMDR research: A control study of sertraline combined with the EMDR in the treatment of depression. Journal of Clinical Psychosomatic Disease, 13(4), 307-308.

Lewey, J. H., Smith, C. L., Burcham, B., Saunders, N. L., Elfallal, D., \& O’Toole, S. K. (2018). Comparing the effectiveness of EMDR and TF-CBT for children and adolescents: A meta-analysis. Journal of Child \& Adolescent Trauma, 11, 457-472. https://doi.org/10.1007/s40653-018-0212-1

Lief, H. I., \& Fetkewicz, J. (1995). Retractors of false memories: The evolution of pseudomemories. The Journal of Psychiatry \& Law, 23(3), 411-435. https://doi.org/10.1177/009318539502300305

Lilley, S. A., Andrade, J., Turpin, G., Sabin-Farrell, R., \& Holmes, E. A. (2009). Visuospatial working memory interference with recollections of trauma. British Journal of Clinical Psychology, 48(3), 309- 


\section{1. https://doi.org/10.1348/014466508X398943}

Linden, M. (2013). How to define, find and classify side effects in psychotherapy: From unwanted events to adverse treatment reactions. Clinical Psychology and Psychotherapy, 20(4), 286-296. https://doi.org/10.1002/cpp.1765

Linden, M., \& Schermuly-Haupt, M. L. (2014). Definition, assessment and rate of psychotherapy side effects. World Psychiatry, 13(3), 306-309. https://doi.org/10.1002/wps.20153

Lindsay, D. S., \& Johnson, M. K. (2000). False memories and the source monitoring framework. Reply to Reyna and Lloyd (1997). Learning and Individual Differences, 12(2), 145-161. https://doi.org/ 10.1016/S1041-6080(01)00035-8

Lilienfeld, S. O. (1996). EMDR treatment: Less than meets the eye? Skeptical Inquirer, 20(1), 25-31.

Lilienfeld, S. O. (2007). Psychological treatments that cause harm. Perspectives on Psychological Science, 2(1), 53-70. https://doi.org/10.1111/j.1745-6916.2007.00029.x

Lilienfeld, S. O., Lynn, S. J., \& Beyerstein, B. L. (2010). The five great myths of popular psychology: Implications for psychotherapy. In: D. David, S. J. Lynn, \& A. Ellis (Eds.), Rational and irrational beliefs: Research, theory, and clinical practice (pp. 313-336). New York, NY, US: Oxford University Press.

Lilienfeld, S. O., Lynn, S. J., Ruscio, J., \& Beyerstein, B. L. (2010). 50 great myths of popular psychology. Shattering widespread misconception about human behavior. Oxford, England: Wiley-Blackwell.

Littel, M., \& van Schie, K. (2019). No evidence for the inverted U-curve: More demanding tasks cause stronger aversive memory degradation. Journal of Behavior Therapy and Experimental Psychiatry, 65, 101484. https://doi.org/10.1016/j.jbteo.2019.101484

Littel, M., Remijn, M., Tinga, A. M., Engelhard, I. M., \& van den Hout, M. A. (2017). Stress enhances the memory-degrading effects of eye movements on emotionally neutral memories. Clinical Psychological Science, 5(2), 316-324. https://doi.org/10.1177/2167702616687292

Littel, M., van Schie, K., \& van den Hout, M. A. (2017). Exploring expectation effects in EMDR: Does prior treatment knowledge affect the degrading effects of eye movements on memories? European Journal of Psychotraumatology, 8, 1328954. https://doi.org/10.1080/20008198.2017.1328954

Loftus, E. F. (1992). When a lie becomes memory's truth: Memory distortion after exposure to misinformation. Current Directions in Psychological Science, 1(4), 121-123. https://doi.org/ 10.1111/1467-8721.ep10769035

Loftus, E. F. (1993). The reality of repressed memories. American Psychologist, 48(5), 518-537. https://doi.org/10.1037/0003-066X.48.5.518

Loftus, E. F. (2004). Memories of things unseen. Current Directions in Psychological Science, 13(4), $145-$ 


\section{References}

147. https://doi.org/10.1111/j.0963-7214.2004.00294.x

Loftus, E. F. (2005). Planting misinformation in the human mind: A 30-year investigation of the malleability of memory. Learning \& Memory, 12, 361-366. https://doi.org/10.1101/Im.94705

Loftus, E. F., \& Burns, T. (1982). Mental shock can produce retrograde amnesia. Memory and Cognition, 10(4), 318-323. https://doi.org/10.3758/BF03202423

Loftus, E. F., \& Davis, D. (2006). Recovered memories. Annual Review of Clinical Psychology, 2, 469-498. https://doi.org/10.1146/annurev.clinpsy.2.022305.095345

Loftus E. F., \& Pickrell, J. E. (1995). The formation of false memories. Psychiatric Annals, 25(12), 720-725. https://doi.org/10.3928/0048-5713-19951201-07

Loftus, E. F., \& Teitcher, J. (2019). Invasion of the mind snatchers: A nation full of traumatic memories. Clinical Psychological Science, 7(1), 25-26. https://doi.org/10.1177/2167702618797107

Loftus, E. F., Doyle, J. M., \& Dysart, J. (2013). Eyewitness testimony: Civil \& criminal (5 $5^{\text {th }}$ ed.). Charlottesville, VA: Leis Law.

Loftus, E. F., Miller, D. G., \& Burns, H. J. (1978). Semantic integration of verbal information into a visual memory. Journal of Experimental Psychology: Human Learning and Memory, 4(1), 19-31. https://doi.org/10.1037/0278-7393.4.1.19

Lohr, J. M., Tolin, D. F., \& Lilienfeld, S. O. (1998). Efficacy of eye movement desensitization and reprocessing: Implications for behavior therapy. Behavior therapy, 29(1), 123-156. https://doi.org/10.16/ S0005-7894(98)80035-X

Lozito, J. P., \& Mulligan, N. W. (2006). Exploring the role of attention during memory retrieval: Effects of semantic encoding and divided attention. Memory \& Cognition, 34(5), 986-998. https://doi.org/10.3758/BF03193246

Lyle, K. B., \& Jacobs, N. E. (2010). Is saccade-induced retrieval enhancement a potential means of improving eyewitness evidence? Memory, 18(6), 581-592. https://doi.org/10.1080/0965821 1.2010 .493891

Lyle, K. B., \& Martin, J. M. (2010). Bilateral saccades increase intrahemispheric but not interhemispheric interaction: Implications for saccade-induced retrieval enhancement. Brain \& Cognition, 73 (2), 128-134. https://doi.org/10.1016/j.bandc.2010.04.004

Magnussen, S, \& Melinder, A. (2012). What psychologists know and believe about memory: A survey of practitioners. Applied Cognitive Psychology, 26(1), 54-60. https://doi.org/10.1002/acp.1795

Marcus, S. V. (2008). Phase 1 of integrated EMDR: An abortive treatment for migraine headaches. Journal of EMDR: Practice \& Research, 2(1), 15 - 25. https://doi.org/10.1891/1933-3196.2.1.15

Mather, M., Henkel, L. A., \& Johnson, M. K. (1997). Evaluating characteristics of false memories: 
Remember/know judgments and memory characteristics questionnaire compared. Memory \& Cognition, 25, 823-837. https://doi.org/10.3758/BF03211327.

Matthijssen, S. J. M. A., Verhoeven, L. C. M., van den Hout, M. A., \& Heitland, I. (2017). Auditory and visual memories in PTSD patients targeted with eye movements and counting: The effect of modality-specific loading of working memory. Frontiers in Psychology, 8, 1937. https://doi.org/ 10.3389/fpsyg.2017.01937

Matzke, D. Nieuwenhuis, S., van Rijn, H., Slagter, H. A., van der Molen, M. W., Wagenmakers, E.-J. (2015). The effect of horizontal eye movements on free recall: A preregistered adversarial collaboration. Journal of Experimental Psychology: General, 144(1), 1-15. https://doi.org/ $10.1037 /$ xge0000038

Mauna Gauhar, Y. W. (2016). The Efficacy of EMDR in the Treatment of Depression. Journal of EMDR Practice and Research, 10(2), 59-69. https://doi.org/10.1891/1933-3196.10.2.59

Mavranezouli, I., Megnin-Viggars, O., Daly, C., Dias, S., Welton, N. J., Stockton, S., ... \& Pilling, S. (2020). Psychological treatment for post-traumatic stress disorder in adults: A network meta-analysis. Psychological Medicine, 50(4), 542-555. https://doi.org/10.1017/S0033291720000070

Mazolla, A., Calcagno, M. L., Goicochea, M. T., Pueyrredòn, H., Leston, J., \& Salvat, F. (2009). EMDR in the treatment of chronic pain. Journal of EMDR Practice and Research, 3(2), 66-79. https://doi.org/10.1891/1933-3196.3.2.66

McCloskey, M., \& Zaragoza, A. (1985). Misleading postevent information and memory for events: Arguments and evidence against memory impairment hypothese. Journal of Experimental Psychology: General, 114(1), 1-16. https://doi.org/10.1037/0096-3445.114.1.1

McGoldrick, T., Begum, M., \& Brown, K. W. (2008). EMDR and olfactory reference syndrome: A case series. Journal of EMDR Practice and Research, 2(1), 63-68. https://doi.org/10.1891/ 1933-3196.2.1.63

McHugh, P. R. (2003). The end of a delusion: The psychiatric memory wars are over. Weekly Standard, 36, 31-34. https://doi.org/10.1097/01.nmd.0000136301.18598.52

McNally, R. J. (1999). EMDR and mesmerism: A comparative historical analysis. Journal of Anxiety Disorders, 13(1), 225-236. https://doi.org/10.1016/S0887-6185(98)00049-8

McNally, R. J. (2003). Recovering memories of trauma: A view from the laboratory. Psychological Science, 12(1), 32-35. https://do.org/:10.1111/1467-8721.01217

McNally, R. J. (2005). Debunking myths about trauma and memory. Canadian Journal of Psychiatry, 50 (13), 817-822. https://doi.org/10.1177/070674370505001302

McNally, R. J. (2016). The expanding empire of psychopathology: The case of PTSD. Psychological Inquiry, 


\section{References}

27(1), 46-49. https://doi.org/10.1080/1047840X.2016.1108168

McNally, R. J., \& Gerearts, E. (2009). A new solution to the recovered memory debate. Perspectives on Psychological Science, 4(2), 126-134. https://doi.org/10.1111/j.1745-6924.2009.01112.x

Merckelbach, H. Smeets, T., Geraerts, E., Jelicic, M., Bouwen, A., \& Smeets, E. (2006). I haven't thought about this for years! Dating recent recalls of vivid memories. Applied Cognitive Psychology, 20(1), 33-42. https://doi.org/10.1002/acp.1153

*Mertens, G., Krypotos, A.-M., van Logtestijn, A., Landkroon, E., van Veen, S. C., \& Engelhard, I. M. (2019). Changing negative autobiographical memories in the lab: A comparison of tree eyemovement tasks. Memory, 27(3), 295-305. https://doi.org/10.1080/09658211.2018.1507041

Mertens, G., van Schie, K., Lammertink, S., Littel, M., \& Engelhard, I. M. (2020). Verbal suggestions about treatment effectiveness do not modulate the effectiveness of a laboratory model of EMDR therapy: Results of two preregistered studies. PsyArXiv Preprints. https://doi.org/ 10.31234/osf.io/zhcp

Miller, S. D., Duncan, B. L., Brown, J., Sparks, J. A., \& Claud, D. (2003). The outcome ratings scale: A preliminary study of the reliability, validity and feasibility of a brief visual analogue measure. Journal of Brief Therapy, 2(2), 91-100.

van Minnen, A., van der Vleugel, B. M., van der Berg, D. P. G., de Bont, P., de Roos, C., van der Gaag, M., et al. (2016). Effectiveness of traumafocused treatment for patients with psychosis with and without the dissociative subtype of post-traumatic stress disorder. British Journal of Psychiatry, 209(4), 347-348. https://doi.org/10.1192/bjp.bp.116.185579

Mitchell, K. J., \& Johnson, M. K. (2000). Source monitoring: Attributing mental experiences. In E. Tulving \& F. I. M. Craik (Eds.), The Oxford handbook of memory (pp. 179-195). New York, NY: Oxford University Press.

Moher, D., Liberati, A., Tetzlaff, J., Altman, D. G., \& Group, P. (2009). Preferred reporting items for systematic reviews and meta-analyses: The PRISMA statement. Annals of Internal Medicine, 151, $264-$ 269. https://doi.org/10.1371/journal.pmed.1000097

Moradi, A. R., Heydari, A. H., Abdollahi, M. H., Rahimi-Movaghar, V., Dalgleish, T., \& Jobson, L. (2015). Visual false memories in posttraumatic stress disorder. Journal of Abnormal Psychology, 124 (4), 905-917. https://doi.org/10.1037/abn0000109

Moreno-Alcázar, A., Treen, D., Valiente-Gómez, A., Sio-Eroles, A., Pérez, V., Amann, B. L., \& Radua, J. (2017). Efficacy of eye movement desensitization and reprocessing in children and adolescents with post-traumatic stress disorder: A meta-analysis of randomized controlled trials. Frontiers in Psychology, 8, 1750. https://doi.org/10.3389/fpsyg.2017.01750 
Morey, R. D., \& Lakens, D. (2016). Why most of psychology is statistically unfalsifiable. Found at https:// github.com/richardmorey/psychology_resolution

Morgan, C. A., III, Southwick, S., Steffian, G., Hazlett, G. A., \& Loftus, E. F. (2013). Misinformation can influence memory for repeatedly experienced, highly stressful events. International Journal of Law \& Psychiatry, 36(1), 11-17. https://doi.org/10.1016/j.ijlp.2012.11.002

Moritz, S., Fieker, M., Hottenrott, B., Seeralan, T., Cludius, B., Kolbeck, K., ..., \& Nestoriuc, Y. (2015). No pain, no gain? Adverse effects of psychotherapy in obsessive-compulsive disorder and its relationship to treatment gains. Journal of Obsessive-Compulsive and Related Disorders, 5 , 61-66. https://doi.org/10.1016/j.jocrd.2015.02.002

Moritz, S., Nestoriuc, Y., Rief, W., Klein, J. P., Jelinek, L., \& Peth, J. (2019). It can't hurt, right? Adverse effects of psychotherapy in patients with depression. European Archives of Psychiatry and Clinical Neuroscience, 269(5), 577-586. https://doi.org/10.1007/s00406-018-093101

Muris, P., \& Merckelbach, H. (1999). Traumatic memories, eye movements, phobia, and panic: A critical note on the proliferation of EMDR. Journal of Anxiety Disorders, 13(1-2), 209-223. https://doi.org/10.1016/S0887-6185(98)00048-6

Muris, P., Merckelbach, H., Holdrinet, I., \& Sijsenaar, M. (1998). Treating phobic children: Effects of EMDR versus exposure. Journal of Consultancy and Clinical Psychology, 66(1), 193-198. https://doi.org/10.1037//0022-006.x.66.1.193

Nahleen, S., Strange, D., \& Takarangi, M. K. T. (2020). Does emotional or repeated misinformation increase memory distortiong for a trauma analogue event? Psychological Research. https://doi.org/10.1007/s00426-020-01409-x

Nazari, H., Momeni, N., Jariani, M., \& Tarrahi, M. J. (2011). Comparison of eye movement desensitization and reprocessing with citalopram in treatment of obsessive-compulsive disorder. International Journal of Psychiatry in Clinical Practice, 15(4), 270-274. https://doi.org/10.3109/13651501. 2011.590210

Nierop, N. M., \& van den Eshof, P. (2010). Herinneringen: Continu, sluimerend, hervonden of gelogen? Ervaringen van de Landelijke Expertisegroep Bijzondere zedenzaken [Memories: Continuous, latent, reovered, or feigned?]. Tijdschrift voor Psychotherapie, 36, 148-170.

Nieuwenhuis, S., Elzinga, B. A., Ras, P. H., Berends, F., Duijs, P., Samara, Z., \& Slagter, H. A. (2013). Bilateral saccadic eye movements and tactile stimulation, but not auditory stimulation, enhance memory retrieval. Brain and Cognition, 81(1), 52-56. https://doi.org/10.1016/j.bandc.2012.10.003

Novo, P., Landin-Romero, R., Radua, J., Vicens, V., Fernandez, I., Garcia, F., et al. (2014). Eye movement desensitization and reprocessing therapy in subsyndromal bipolar patients with a history of 


\section{References}

traumatic events: A randomized, controlled pilot-study. Psychiatry Research, 219(1), 122-128. https://doi.org/10.1016/j.psychres.2014.05.012

Odinot, G., Boon, R., \& Wolters, L. (2015). Het episodisch geheugen en getuigenverhoor [Episodic memory and witness examination]. Tijdschrift voor Criminologie, 57, 279-299. doi:10.5553/ TvC/0165182X2015057003001

Onderdonk, S. W., \& van den Hout, M. A. (2016). Comparisons of eye movements and matched changing visual input. Journal of Behavior Therapy and Experimental Psychiatry, 53, 34-40. https://doi.org/10.1016/j.jbtep.2015.10.010

Orbach, Y., \& Lamb, M. E. (1999). Assessing the accuracy of a child's account of sexual abuse: A case study. Child Abuse \& Neglect, 23(1), 91-98. https://doi.org/10.1016/S0145-2134(98)00114-8

Ost, J., Costall, A., \& Bull, R. (2001). False confessions and false memories: A model for understanding retractors' experiences. Journal of Forensic Psychiatry, 12(3), 549-579. https://doi.org/ $10.1080 / 095851801217393$

Ost, J., Costall, A., \& Bull, R. (2002). A perfect symmetry? A study of retractors' experiences of making and then repudiating claims of early sexual abuse. Psychology, Crime, \& Law, 8(2), 155-181. https://doi.org/10.1080/10683160208415004

Ost, J., Wright, D., Easton, S., Hope, L., \& French, C. (2013). Recovered memories, satanic abuse, dissociative identity disorder, and false memories in the U.K.: A survey of clinical psychologists and hypnotherapists. Psychology, Crime \& Law, 19(1), 1-19. https://doi.org/10.1080/ 1068316X.2011.598157

Otgaar, H., Candel, I., \& Merckelbach, H. (2008). Children's false memories: Easier to elicit for a negative than for a neutral event. Acta Psychologica, 128(2), 350-354. https://doi.org/10.1016/ j.actpsy.2008.03.009

Otgaar, H., Candel, I., Merckelbach, H., \& Wade, K. A. (2009). Abducted by a UFO: Prevalence information affects young children's false memories for an implausible event. Applied Cognitive Psychology, 23(1), 115-125. https://doi.org/10.1002/acp.1445

Otgaar, H., Howe, M. L., Brackmann, N., \& Smeets, T. (2016). The malleability of developmental trends in neutral and negative memory illusions. Journal of Experimental Psychology: General, 145(1), 31-55. https://doi.org/10.1037/xge0000127

Otgaar, H., Howe, M. L., Merckelbach, H., \& Muris, P. (2018). Who is the better eyewitness? Sometimes adults but at other times children. Current Directions in Psychological Science, 27(5), 378-385. https://doi.org/10.1177/0963721418770998

Otgaar, H., Howe, M. L., Muris, P., \& Merckelbach, H. (2019). Associative activation as a mechanism 
underlying false memory formation. Clinical Psychological Science, 7(2), 191-195.

https://doi.org/10.1177/2167702618807189

Otgaar, H., Howe, M. L., Patihis, L., Merckelbach, H., Lynn, S. J., Lilienfeld, S. O., \& Loftus, E. F. (2019). The return of the repressed: The persistent and problematic claims of long-forgotten trauma. Perspectives on Psychological Science, 14(6), 1072-1095. https://doi.org/10.1177/ 1745691619862306

Otgaar, H., Howe, M. L., Peters, M., Sauerland, M., \& Raymaekers, L. (2013). Developmental trends in different types of spontaneous false memories: Implications for the legal field. Behavioral Sciences and the Law, 31, 666-682. https://doi.org/10.1002/bsl.2076

Otgaar, H., Peters, M., \& Howe, M. L. (2012). Dividing attention lowers children's but increases adult's false memories. Journal of Experimental Psychology, 38(1), 204-210. https://doi.org/ $10.1037 / \mathrm{a} 0025160$

Otgaar, H., Muris, P., Howe, M. L., \& Merckelbach, H. (2017). What drives false memories in psychopathology? A case for associative activation. Clinical Psychological Science, 5(6), 1048-1069. https://doi.org/10.1177/2167702617724424

Otgaar, H., Scoboria, A., Howe, M. L., Moldoveanu, G., \& Smeets, T. (2016). Challenging memories in children and adults using an imagination inflation procedure. Psychology of Consciousness: Theory, Research, and Practice, 3(3), 270-283. https://doi.org/10.1037/cns0000087

Otgaar, H., Wang, J., Howe, M. L., Lilienfeld, S. O., Loftus, E. F., Lynn, S. J., ... Patihis, L. (2019). Belief in unconscious repressed memory is wide-spread: A comment on Brewin, Li, Ntarantana, Unsworth, and McNeilis. Journal of Experimental Psychology: General. https://doi.org/ 10.31219/osf.io.a4n7h

Owen, J., Drinane, J. M., Idigo, K. C., \& Valentine, J. C. (2015). Psychotherapist effects in meta-analyses: How accurate are treatment effects? Psychotherapy, 52(3), 321-328. https://doi.org/10.1037/ pst0000014

Paris, J. (2012). The rise and fall of dissociative identity disorder. Journal of Nervous and Mental Disease, 200(12), 1076-1079. https://doi.org/10.1097/NMD.0b013e318275d285

Parker, A., \& Dagnall, N. (2007). Effect of bilateral eye movements on gist based false recognition in the DRM paradigm. Brain and Cognition, 63(3), 221-225. https://doi.org/10.1016/j.bandc.2006.08.005

Parker, A., \& Dagnall, N. (2012). Effects of saccadic bilateral eye movements on memory in children and adults: An exploratory study. Brain and Cognition, 78(3), 238-247. https://doi.org/10.1016/ j.bandc.2012.01.007

Parker, A., Buckley, S., \& Dagnall, N. (2009). Reduced misinformation effects following saccadic bilateral 


\section{References}

eye movements. Brain and Cognition, 69(1), 89-97. https://doi.org/10.1016/j.bandc.2008.05.009

Parker, A., Poole, J., \& Dagnall, N. (2020). Saccade-induced retrieval enhancement and the recovery of perceptual item-specific information. Cognitive Processing, 21, 223-237. https://doi.org/ s10339-019-00943-w

Parry, G.D., Crawford, M.J., \& Duggan, C. (2016). latrogenic harm from psychological therapies - Time to move on. The British Journal of Psychiatry, 208(3), 210-212. https://doi.org/10.1192/ bjp.bp.115.163618

Passoni, S., Curinga, T., Toraldo, A., Berlingeri, M., Fernandez, I., \& Bottini, G. (2018). Eye movement desensitization and reprocessing integrative group treatment protocol (EMDR-IGTP) applied to caregivers of patients with dementia. Frontiers in Psychology, 9, 967. https://doi.org/ 10.3389/fpsyg.2018.00967

Patihis, L. (2018). Why there is no false memory trait and why everyone is susceptible to memory distortions: The dual encoding interference hypothesis (commentary on Bernstein, Scoboria, Desjarlais, \& Soucie, 2018). Psychology of Consciousness: Theory, Research, and Practice, 5 (2), 180-184. https://doi.org/10.1037/cns0000143

Patihis, L., \& Pendergrast, M. H. (2019). Reports of recovered memories of abuse in therapy in a large age-representative U.S. nation sample: Therapy type and decade comparisons. Clinical Psychological Science, 7(1), 3-21. https://doi.org/10.1177/2167702618773315

Patihis, L., Frenda, S. J., \& Loftus, E. F. (2018). False memory tasks do not reliably predict other false memories. Psychology of Consciousness: Theory, Research, and Practice, 5(2), 140-160. https://doi.org/10.1037/cns0000147

Patihis, L., Ho, L. Y., Loftus, E. F., \& Herrera, M. E. (2018). Memory experts' beliefs about repressed memory. Memory, 26. https://doi.org/10.1080/09658211.2018.1532521

Patihis, L., Ho, L. Y., Tingen, I. W., Lilienfeld, S. O., \& Loftus, E. F. (2014). Are the "memory wars" over? A scientist-practitioner gap in beliefs about repressed memories. Psychological Science, 25(2), 519-530. https://doi.org/10.1177/0956797613510718

Patihis, L., Otgaar, H., \& Merckelbach, H. (2019). Expert witnesses, dissociative amnesia, and extraordinary remembering: Response to Brand et al. Psychological Injury and Law, 12, 281-285. https://doi.org/10.1007/s12207-019-09348-8

Peace, K. A., \& Porter, S. (2004). A longitudinal investigation of the reliability of memories for trauma and other emotional experiences. Applied Cognitive Psychology, 18(9), 1143-1159. https://doi.org/10.1002/acp.1046

Pearson, D. G., Ross, F. D. C., \& Webster, V. L. (2012). The importance of context: Evidence that contextual 
representations increase intrusive memories. Journal of Behavior Therapy and Experimental Psychiatry, 43(1), 573-580. https://doi.org/10.1016/j.jbtep.2011.07.009

Perez-Dandieu, B., \& Tapia, G. (2014). Treating trauma in addiction with EMDR: A pilot study. Journal of Psychoactive Drugs, 46(4), 303-309. https://doi.org/10.1080/02791072.2014.921744

Pesta, B. J., Murphy, M. D., \& Sanders, R. E. (2001). Are emotionally charged lures immune to false memory? Journal of Experimental Psychology: Learning, Memory, and Cognition, 27(2), 328338. https://doi.org/10.1037//0278-7393.27.2.328

Peters, M. J. V., Jelicic, M., Verbeek, H., \& Merckelbach, H. (2007). Poor working memory predicts false memories. European Journal of Cognitive Psychology, 19(2), 213-232. https://doi.org/ 10.1080/09541440600760396

Pezdek, K., Blandon-Gitlin, I., Lam, S., Hart, R. E., \& Schooler, J. W. (2006). Is knowing believing? The role of event plausibility and background knowledge in planting false beliefs about the personal past. Memory \& Cognition, 34, 1628-1635. https://doi.org/10.3758/BF03195925

Pezdek, K., Finger, K., \& Hodge, D. (1997). Planting false childhood memories: The role of event plausibility. Psychological Science, 8(6), 437-441. https://doi.org/10.1111/j.1467-9280.1997.tb00457.x

Phelps, E. A., \& Hofmann, S. G. (2019). Memory editing from science fiction to clinical practice. Nature, 572, 43-50. https://doi.org/10.1038/s41586-019-1433-7

Porter, S., Spencer, L., \& Birt, A. R. (2003). Blinded by emotion? Effect of the emotionality of a scene on susceptibility to false memories. Canadian Journal of Behavioural Science, 35(3), 165-175. https://doi.org/10.1037/h0087198

Powers, P. A., Andriks, J. L., \& Loftus, E. F. (1979). The eyewitness accounts of females and males. Journal of Applied Psychology, 64(3), 339-347. https://doi.org/10.1037/0021-9010.64.3.339

Propper, R. E., \& Christman, S. D. (2008). Interhemispheric interaction and saccadic horizontal eye movements. Journal of EMDR Practice and Research, 2(4), 269-280. https://doi.org/10.1891/ 1933-3196.2.4.269

Qualtrics software, Version December 2017 of Qualtrics, Copyright (C2018 Qualtrics. Qualtrics and all other Qualtrics product or service names are registered trademarks or trademarks of Qualtrics, Provo, UT, USA. https://www.qualtrics.com

Quintana, D. S. (2015). From pre-registration to publication: A non-technical primer for conducting meta-analysis to synthesize correlational data. Frontiers in Psychology, 6:1549. https://doi.org/10.3389/fpsyg.2015.01549

Rahimi, F., Rejeh, N., Bahrami, T., Heravi-Karimooi, M., Tadrisi, S. D., Griffiths, P., \& Vaismoradi, M. (2018). The effect of the eye movement desensitization and reprocessing intervention on anxiety and 


\section{References}

depression among patients undergoing hemodialysis: A randomized controlled trial. Perspectives in Psychiatric Care, 55(4), 652-660. https://doi.org/10.1111/ppc.12389

Rathschlag, M., \& Memmert, D. (2014). Reducing anxiety and enhancing physical performance by using an advanced version of EMDR: A pilot study. Brain and Behavior, 4(3), 348-355. https://doi.org/10.1002/brb3.221

Ray, P., \& Page, A. C. (2002). A single session of hypnosis and eye movement desensitisation and reprocessing (EMDR) in the treatment of chronic pain. Australian Journal of Clinical and Experimental Hypnosis, 30(2), 170-178.

Raymaekers, L., Peters, M. J. V., Smeets, T., Abidi, L., \& Merckelbach, H. (2011). Underestimation of prior remembering and susceptibility to false memories: Two sides of the same coin? Consciousness and Cognition, 20(4), 1144-1153. https://doi.org/10.1016/j.concog.2010.12.010

Raymaekers, L., Smeets, T., Peters, M. J. V., Otgaar, H., \& Merckelbach, H. (2012). The classification of recovered memories: A cautionary note. Consciousness and Cognition, 21(4), 1640-1643. https://doi.org/10.1016/.j.concog.2012.09.002

Reefhuis, E. R., van Dijk, M. K., Miggiels, M. F., ten Klooster, P. M., Huibers, M. J. H., \& Dekker, J. J. M. (2019). De kwaliteit van de therapeutische relatie voorspelt uitkomst van psychotherapie bij depressie [the quality of the therapeutic relationship predicts therapy results in depression]. Tijdschrift voor Psychotherapie, 45, 6-23.

Rikkert, M., van Rood, Y., de Roos, C., Ratter, J., \& van den Hout, M. (2018). A trauma-focused approach for patients with tinnitus: The effectiveness of eye movement desensitization and reprocessing - a multicentre pilot trial. European Journal of Psychotraumatology, 9:1, 1512248. https://doi.org/10.1080/20008198.2018.1512248

Roberts, B. R. T., Fernandes, M. A., \& MacLeod, C. M. (2020). Re-evaluating whether bilateral eye movements influence memory retrieval. PLOS ONE, 15(1), e0227790. https://doi.org/10.1371/ journal.ppone.0227790

Rodenburg, R., Benjamin, A., de Roos, C., Meijer, A. M., \& Stams, G. J. (2009). Efficacy of EMDR in children: A meta-analysis. Clinical Psychological Review, 29(7), 599-606. https://doi.org/10.1016/ j.cpr.2009.06.008

Roediger, H. L., \& McDermott, K. (1995). Creating false memories: Remembering words not presented in a list. Journal of Experimental Psychology: Learning, Memory, and Cognition, 21(4), 803814. https://doi.org/10.1037/0278-7393.21.4.803

Roediger, H. L., Balota, D. A., \& Watson, J. M. (2001). Spreading activation and the arousal of false memories. In: H. L. Roediger, J. S. Nairne, I. Neath, \& A. M. Surprenant (Eds.), The nature of 
remembering: Essays in honor of Robert G. Crowder (pp. 95-115). Washington, DC: American Psychological Association.

Roediger, H. L., Watson, J. M., McDermott, K. B., \& Gallo, D. A. (2001). Factors that determine false recall: A multiple regression analysis. Psychonomic Bulletin \& Review, 8, 385-407. https://doi.org/ 10.3758/BF03196177

Rofé, Y. (2008). Does repression exist? Memory, pathogenic, unconscious and clinical evidence. Review of General Psychology, 12(1), 63-85. https://doi.org/10.1037/1089-2680.12.1.63

van Rood, Y. R., \& de Roos, C. (2009). EMDR in the treatment of medically unexplained symptoms: A systematic review. Journal of EMDR Practice and Research, 3(4), 248-263. https://doi.org/ 10.1891/1933-3196.3.4.248

de Roos, C., Veenstra, A. C., de Jongh, A., den Hollander-Gijsman, M. E., van der Wee, N. J. A., \& van Rood, Y. R. (2010). Treatment of chronic phantom-limb-pain (PLP) using a trauma focused psychological approach: Ten cases. Pain research and management, 15(2), 65-71. https://doi.org/10.11155/2010/981634

Rosenthal, R., Kohn, P., Greenfield, P. M., \& Carota, N. (1966). Data desirability, experimenter expectancy, and the results of psychological research. Journal of Personality and Social Psychology, 3(1), 20-27. https://doi.org/10.1037/h0022604

Royle, L. (2008). EMDR as a therapeutic treatment for chronic fatigue syndrome (CFS). Journal of EMDR Practice and Research, 2(3), 226-232. https://doi.org/10.1891/1933-3196.2.3.226

Rozental, A., Kottorp, A., Boettcher, J., Andersson, G., \& Carlbring, P. (2016). Negative effects of psychological treatments: An exploratory factor analysis of the negative effects questionnaire for monitoring and reporting adverse and unwanted events. PLOS ONE, 11, e0157503. https://doi.org/ 10.1371/journal.pone.0157503

Rubin, D. C., \& Kozin, M. (1984). Vivid memories. Cognition, 16(1), 81-95. https://doi.org/10.1016/ 0010-0277(84)90037-4

Russell, M. C. (2008a). Treating traumatic amputationrelated phantom limb pain: A case study utilizing eye movement desensitization and reprocessing within the armed services. Clinical Case Studies, 7(2), 136-153. https://doi.org/10.1177/1534650107306292

Russell, M. C. (2008b). War-related medically unexplained symptoms, prevalence, and treatment: Utilizing EMDR within the armed services. Journal of EMDR Practice and Research, 2(3), 212225. https://doi.org/:10.1891/1933-3196.2.3.212

Sack, M., Zehl, S., Otti, A., Lahmann, C., Henningsen, P., Kruse, J., \& Stingl, M. (2016). A comparison of dual attention, eye movements, and exposure only during eye movement desensitization and 


\section{References}

reprocessing for posttraumatic stress disorder: Results from a randomized controlled trial. Psychotherapy and Psychosomatics, 85(6), 357-365. https://doi.org/10.1159/000447671

Saks, M. J., \& Koehler, J. J. (2005). The coming paradigm shift in forensic identification science. Science, 309(5736), 892-895. https://doi.org/10.1126/science.1111565

Salthouse, T. A., \& Siedlecki, K. L. (2007). An individual difference analysis of false recognition. The American Journal of Psychology, 120(3), 429-458.

Samara, Z., Elzinga, B. M., Slagter, H. A., \& Nieuwenhuis, S. (2011). Do horizontal saccadic eye movements increase interhemispheric coherence? Investigation of a hypothesized neural mechanism underlying EMDR. Frontiers in Psychiatry, 2(4), pmid:21556274. https://doi.org/10.3389/ fpsyt.2011.00004

Schacter, D. L. (1996). Searching for memory: The brain, the mind, and the past. New York (NY): Basic Books.

Scoboria, A., Mazzoni, G., Kirsch, I., \& Jimenez, S. (2006). The effects of prevalence and script information on plausibility, belief, and memory of autobiographical events. Applied Cognitive Psychology, 20(8), 1049-1064. https://doi.org/10.1002/acp/1240

Scoboria, A., Wade, K. A., Lindsay, D. S., Azad, T., Strange, D., Ost, J., \& Hyman, I. E. (2017). A mega-analysis of memory reports from eight peer-reviewed false memory implantation studies. Memory, 25(2), 146-163. https://doi.org/10.1080-09658211.2016.1260747

van Schie, K., \& Leer, A. (2019). Lateral eye movements do not increase false memories rates: A failed direct replication study. Clinical Psychological Science, 7(5), 1159-1167. https://doi.org/ $10.1177 / 2167702619859335$

van Schie, K., Engelhard, I. M., \& van den Hout, M. A. (2015). Taxing working memory during retrieval of emotional memories does not reduce memory accessibility when cued with reminders. Fronties in Psychiatry, 6, 16. https://doi.org/10.3389/fpsyt.2015.00016

van Schie, K., van Veen, S. C., \& Hagenaars, M. A. (2019). The effects of dual-tasks on intrusive memories following analogue trauma. Behavior Research and Therapy, 120, 103448. https://doi.org/ 10.1016/j.brat.2019.103448

*van Schie, K., van Veen, S. C., Engelhard, I. M., Klugkist, I., \& van den Hout, M. A. (2016). Blurring emotional memories using eye movements: Individual differences and speed of eye movements. European Journal of Psychotraumatology, 7: 29476. https://doi.org/10.3402/ejpt.v7.29476

Schneider, J., Hofmann, A., Rost, C., \& Shapiro, F. (2008). EMDR in the treatment of chronic phantom limb pain. Pain Medicine, 9(1), 76-82. https://doi.org/10.1111/j.1526-4637.2007.00299.x

Schooler, J. W. (2001). Discovering memories of abuse in the light of meta-awareness. In J. J. Freyd, \& A. P. DePrince (Eds.), Trauma and Cognitive Science: A meeting of minds, science and human 
experience (pp.105-136). New York: Haworth.

Schooler, J. W., Ambadar, Z., \& Bendiksen, M. (1997). A cognitive corroborative case study approach for investigating discovered memories of sexual abuse. In: J. D. Read, \& D. S. Lindsay (Eds.), Recollections of Trauma. Boston, MA: Springer.

Shaffer, R. E., \& Cozolino, L. J. (1992). Adults who report childhood ritualistic abuse. Journal of Psychology and Theology, 20(3), 188-193. https://doi.org/10.1177/009164719202000302

Shah, D., \& Knott, L. M. (2018). The role of attention at retrieval on the false recognition of valence emotional DRM lists. Memory, 26(2), 269-276. https://doi.org/10.1080/09658211.2017.1349803

Shapiro, F. (1989). Efficacy of the eye movement desensitization procedure in the treatment of traumatic memories. Journal of Traumatic Stress, 2(2), 199-223. https://doi.org10.1002/jts.2490020207

Shapiro, F. (2002). EMDR treatment: Overview and integration. In F. Shapiro (Ed.), EMDR as an integrative psychotherapy approach: Experts of diverse orientations explore the paradigm prism (pp. 2755). Washington, D.C.: American Psychological Association.

Shapiro, F., \& Forrest, M. S. (2004). EMDR: The breakthrough therapy for overcoming anxiety, stress, and trauma. New York, NY: Basic Books.

Shapiro, F., \& Laliotis, D. (2015). EMDR therapy for trauma-related disorders. In U. Schnyder, \& M. Cloitre (Eds.), Evidence based treatments for trauma-related psychological disorders (pp. 205-228). Zurich: Springer.

Shapiro, F., \& Maxfield, L. (2002). Eye movement desensitization and reprocessing (EMDR): Information processing in the treatment of trauma. Psychotherapy in Practice, 58(8), 933-946. https://doi.org/10.1002/jclp.10068.

Shaw, J., \& Porter, S. (2015). Constructing rich false memories of committing crime. Psychological Science, 26(3), 291-301. https://doi.org/10.1177/0956797614562862

Shaw, J., \& Vredeveldt, A. (2019). The recovered memory debate continues in Europe: Evidence from the United Kingdom, the Netherlands, France, and Germany. Clinical Psychological Science, 7(1), 27-28. https://doi.org/10.1177/2167702618803649

Shimokawa, K., Lambert, M. J., \& Smart, D. W. (2010). Enhancing treatment outcome of patients at risk of treatment failure: Meta-analytic and mega-analytic review of a psychotherapy quality assurance system. Journal of Consulting \& Clinical Psychology, 78(3), 298-311. https://doi.org/10.1037/a0019247

Shirk, S. R., Karver, M. S., \& Brown, R. (2011). The alliance in child and adolescent therapy. Psychotherapy, 48(1), 17-24. https://doi.org/10.1037/a0022181

Shobe, K. K., \& Kihlstrom, J. F. (2002). Interrogative suggestibility and 'memory work'. In: M. I. Eisen, G. S., 


\section{References}

Goodman, \& J. Quas. (Eds.), Memory and Suggestibility in the Forensic Interview. Mahwah, N.J.L Erlbaum.

Silver, S. M., Rogers, S., \& Russell, M. (2008). Eye movement desensitization and reprocessing (EMDR) in the treatment of war veterans. Journal of Clinical Psychology: In Session, 64(8), 947-957. https://doi.org/10.1002/jclp.20510

*Smeets, M. A. M., Dijs, M., W., Pervan, I., Engelhard, I. M., \& van den Hout, M. A. (2012). Time-course of eye movement-related decrease in vividness and emotionality of unpleasant autobiographical memories. Memory, 20(4), 346-357. https://doi.org/10.1080/09658211.2012.665462

Soberman, G. B., Greenwald, R., \& Rule, D. L. (2002). A controlled study of eye movement desensitization and reprocessing (EMDR) for boys with conduct problems. Journal of Aggression, Maltreatment \& Trauma, 6(1), 217-236. https://doi.org/10.1300/J146v06n01_11

Solomon, R. M., \& Shapiro, F. (2008). EMDR and the adaptive information processing model: Potential mechanisms of change. Journal of EMDR Practice and Research, 2(4), 315-325. https://doi.org/10.1891/1933-3196.2.4.315

Spiegel, D. (1997). Foreword. In D. Spiegel (Ed.), Review of Psychiatry: Vol. 16, Section II. Repressed memories (pp. 5-11). Washington, DC: American Psychiatric Press.

Staring, A. B. P., van den Berg, D. P. G., Cath, D. C., Schoorl, M., Engelhard, I. M., \& Korrelboom, C. W. (2016). Self-esteem treatment in anxiety: a randomized controlled crossover trial of Eye Movement Desensitization and Reprocessing (EMDR) vs. Competitive Memory Training (COMET) in patients with anxiety disorders. Behaviour Research and Therapy, 82, 11-20. https://doi.org/10.1016/j.brat.2016.04.002

Strange, D., \& Takarangi, M. K. (2012). False memories for missing aspects of traumatic events. Acta Psychologica, 141(3), 322-326. https://doi.org/10.1016/j.actpsy.2012.08.005

Strange, D., Sutherland, R., \& Garry, M. (2006). Event plausibility does not determine children's false memories. Memory, 14(8), 937-951. https://doi.org/10.1080.09658210600896105

Talmi, D., Luk, B. C., McGarry, L. M., \& Moscovitch, M. (2007). The contribution of relatedness and distinctiveness to emotionally-enhanced memory. Journal of Memory and Language, 56(4), 555-574. https://doi.org/10.1016/j.jml.2007.01.002

Tang, T. C., Yang, P., Yen, C. F., \& Liu, T. L. (2015). Eye movement desensitization and reprocessing for treating psychological disturbances in Taiwanese adolescents who experienced Typhoon Morakot. The Kaohsiung Journal of Medical Sciences, 31(7), 363-369. https://doi.org/ 10.1016/j.kjms.2015.04.013

Taylor, A. K., \& Kowalski, P. (2004). Naïve psychological science: The prevalence, strength, and sources of 
misconceptions. Psychological Record, 54, 15-25. https://doi.org/10.1007/BF03395459

Tesarz, J., Leisner, S., Gerhardt, A., Janke, S., Seidler, G. H., Eich, W., \& Hartmann, M. (2014). Effects of eye movement desensitization and reprocessing (EMDR) treatment in chronic pain patients: A systematic review. Pain Medicine, 15(2), 247-263. https://doi.org/10.1111/pme.12303

Thomaes, K., Engelhard, I. M., Sijbrandij, M., Cath, D. C., \& Van den Heuvel, O. A. (2016). Degrading traumatic memories with eye movements: A pilot functional MRI study in PTSD. European Journal of Psychotraumatology, 7, 31371. https://doi.org/ 10.3402/ejpt.v7.31371

Tomes, J. L., \& Katz, A. N. (1997). Habitual susceptibility to misinformation and individual differences in eyewitness memory. Applied Cognitive Psychology, 11(3), 233-251. https://doi.org/10.1002/ (SICI)1099-0720(199706)

Tousignant, J. P., Hall, D., \& Loftus, E. F. (1986). Discrepancy detection and vulnerability to misleading postevent information. Memory \& Cognition, 14, 329-338. https://doi.org/10.3758/BF03202511

Tracey, T. J. G., Wampold, B. E., Lichtenberg, J. W., \& Goodyear, R. K. (2014). Expertise in psychotherapy: An elusive goal? American Psychologist, 69(3), 218-229. https://doi.org/10.1037/a0035099

Triscari, M. T., Faraci, P., Catalisano, D., D’Angelo, V., \& Urso, V. (2015). Effectiveness of cognitive behavioral therapy integrated with systematic desensitization, cognitive behavioral therapy combined with eye movement desensitization and reprocessing therapy, and cognitive behavioral therapy combined with virtual reality expo. Neuropsychiatric Disease and Treatment, 11, 2591-2598. https://doi.org/10.2147/NDT.S93401

Tulving, E. (1985). Memory and consciousness. Canadian Psychology, 26(1), 1-12.https://doi.org/ 10.1037/h0080017

Valiente-Gómez, A., Moreno-Alcázar, A., Treen, D., Cedrón, C., Colom, F., Pérez, V., \& Amann, B. L. (2017). EMDR beyond PTSD: A systematic literature review. Frontiers in Psychology, 8:1668. https://doi.org/10.3389/fpsyg.2017.01668

Van Damme, I., \& Smets, K. (2014). The power of emotion versus the power of suggestion: Memory for emotional events in the misinformation paradigm. Emotion, 14(2), 310-320. https://doi.org/ 10.1037/a0034629

*van Veen, S. C., Engelhard, I. M., \& van den Hout, M. A. (2016). The effects of eye movements on emotional memories: Using an objective measure of cognitive load. European Journal of Psychotraumatology, 7, 30122. https://doi.org/10.3402/ejpt.v7.30122

van Veen, S. C., Kang, S., \& van Schie, K. (2019). On EMDR: Measuring the working memory taxation of various types of eye (non-)movement conditions. Journal of Behavior Therapy and Experimental Psychiatry, 65, 101494. doi:10.1016/j.jbteo.2019.101494 


\section{References}

van Veen, S. C., van Schie, K., van de Schoot, R., van den Hout, M. A., \& Engelhard, I. M. (2020). Making eye movements during imaginal exposure leads to short-lived memory effects compared to imaginal exposure alone. Journal of Behavior Therapy and Experimental Psychiatry, 67, 101466. https://doi.org/10.1016/j.jbtep.2019.03.001

*van Veen, S. C., van Schie, K., Wijngaards-de Meij, L. D., Littel, M., Engelhard, I. M., \& van den Hout, M. A. (2015). Speed matters: Relationship between speed of eye movements and modification of aversive autobiographical memories. Frontiers in Psychiatry, 6:45. https://doi.org/10.3389/ fpsyt.2015.00045

Vervaeke, G., Bogaerts, S., \& Heylen, A. (2002). Onderkennen van seksueel misbruik bij kinderen [Recognizing sexual abuse in children]. In P. J. van Koppen, D. J. Hessing, H. L. G. J. Merckelbach, \& H. F. M. Crombag (Eds.), Het recht van binnen: Psychologie van het recht (pp. 177-200). Deventer: Kluwer.

de Voogd, L. D., Kanen, J. W., Neville, D. A., Roelofs, K., Fernández, G., \& Hermans, E. J. (2018). Eyemovement intervention enhances extinction via amygdala deactivation. The Journal of Neuroscience, 38(40), 8694-8706. https://doi.org/10.1523/JNEUROSCI.0703-18.2018

Wade, K. A., Garry, M., \& Pezdek, K. (2018). Deconstructing rich false memories of committing crime: Commentary on Shaw and Porter (2015). Psychological Science, 29(3), 471-476. https://doi.org/10.1177/0956797617703667

Wade, K. A., Garry, M., Read, J. D., \& Lindsay, D. S. (2002). A picture is worth a thousand lies: Using false photographs to create false childhood memories. Psychonomic Bulletin \& Review, 9, 597-603. https://doi.org/10.3758/BF03196318

Wade, K. A., Sharman, S. J., Garry, M., Memon, A., Mazzoni, G., Merckelbach, H., et al. (2007). False claims about false memory research. Consciousness and Cognition, 16(1), 18-28. https://doi.org/ 10.1016/j.concog.2006.07.001.

Waller, G. (2009). Evidence-based treatment and therapist drift. Behaviour Research and Therapy, 47 (2), 119-127. https://doi.org10.1016/j.brat.2008.10.018

Waller, G., \& Turner, H. (2018). Het terugdringen van therapeutische dwaling: waarom goedwillende clinici er niet in slagen om evidence-based therapie te bieden, en hoe we weer op koers kunnen komen [Reducing therapeutic error: Why well-meaning clinicians fail to provide evidence-based therapy and how to get back on track]. Gedragstherapie, 51, 85-111.

Weede Alexander, K., Quas, J. A., Goodman, G. S., Ghetti, S., Edelstein, R. S., Redlich, A. D., Cordon, I. M., \& Jonger, D. P. H. (2005). Traumatic impact predicts long-term memory for documented child sexualabuse. PsychologicalScience, 16(1), 33-40.https://doi.org/10.1111/j.0956.7976.2005.00777.x 
Went, M. (2016). Workshop EMDR bij preverbal trauma [EMDR in preverbal trauma]. Retrieved from https://www.psy-zo.nl/onderwijs/opleidingen/emdrbij-preverbaal-trauma/?instance=239\&cs=ea8bd

Werbart, A., Annevall, A., \& Hillblom, J. (2019). Successful and less successful psychotherapies compared: Three therapists and their six contrasting cases. Frontiers in Psychology, 10:816. https://doi.org/10.3389/fpsyg.2019.00816

Werbart, A., von Below, C., Brun, J., \& Gunnarsdottir, H. (2015). "Spinning one's wheels": Nonimproved patients view their psychotherapy. Psychotherapy, 25(5), 546-564. https://doi.org/10.1080/ 10503307.2014.989291

Werbart, A., von Below, C., Engqvist, K., \& Lind, S. (2018). "It was like having half of the patient in therapy": Therapists of nonimproved patients looking back on their work. Psychotherapy Research, 29 (7), 894-907. https://doi.org/ 10.1080/1050330 7.2018.1453621

Werbart, A., Missios, P., Waldenström, F., \& Lilliengren, P. (2019). "It was hard work every session": Therapists' view of successful psychoanalytic treatments. Psychotherapy Research, 29(3), 354-371. https://doi.org/10.1080/10503307.2017.1349353

Wessel, I., Albers, C. J., Zandstra, A. R. E., \& Heininga, V. E. (2020). A multiverse analysis of early attempts to replicate memory suppression with the Think/No-think Task. Memory, 28(7). https://doi.org/10.1080/09658211.2020.1797095

Wessel, I., Raymaekers, L., Nierop, N., van den Eshof, P., Otgaar, H., \& Jelicic, M. (2017). Hervonden herinneringen. In: P. J. van Koppen, J. W. de Keijser, R. Horselenberg, \& M. Jelicic (Eds.), Routes van het Recht: Over de rechtspsychologie. Den Haag: Boom Juridisch, pp.553-571.

Wilensky, M. (2006). Eye movement desensitization and reprocessing (EMDR) as a treatment for phantom limb pain. Journal of Brief Therapy, 5(1), 31-44.

Wilson, S. A., Becker, L. A., \& Tinker, R. H. (1995). Eye movement desensitization and reprocessing (EMDR) treatment for psychologically traumatized individuals. Journal of Consulting and Clinical Psychology, 63(6), 928-937. https://doi.org/10.1037/0022-006X.63.6.928

Wilson, G., Farrell, D., Barron, I., Hutchins, J., Whybrow, D., \& Kiernan, M. D. (2018). The use of eyemovement desensitization and reprocessing (EMDR) therapy in treating post-traumatic stress disorder - a systematic narrative review. Frontiers in Psychology, 9:923. https://doi.org/10.3389/fpsyg.2018.00923

Yapko, M. D. (1994). Suggestibility and repressed memories of abuse: A survey of psychotherapists' beliefs. American Journal of Clinical Hypnosis, 36(3), 163-171. https://doi.org/10.1080/ 00029157.1994 .10403066

Yunitri, N., Kao, C.-C., Chu, H., Voss, J., Chiu, H.-L., Liu, D., Shen, S.-T. L., Chang, P.-C., Kang, X. L., \& Chou, 


\section{References}

K.-R. (2020). The effectiveness of eye movement desensitization and reprocessing toward anxiety disorder: A meta-analysis of randomized controlled trials. Journal of Psychiatric Research, 123, 102-113. https://doi.org/ 10.1016/j.jpsychires.2020.01.005

Zaccagnino, M., Cussino, M., Callerame, C., Civilotti, C., \& Fernandez, I. (2017). Anorexia nervosa and EMDR: A clinical case. Journal of EMDR Practice and Research, 11(1), 43-53. https://doi.org/10.1891/1933-3196.11.1.43

Zaragoza, M. S., \& Mitchell, K. J. (1996). Repeated exposure to suggestion and the creation of false memories. Psychological Science, 7(5), 294-300. https://doi.org/10.1111/j.1467-9280. 1996.tb00377.x

Zaragoza, M. S., Belli, R. F., \& Payment, K. E. (2006). Misinformation effects and the suggestibility of eyewitness memory. In: M. Garry, \& H. Hayne (Eds.), Do justice and let the sky fall: Elizabeth F. Loftus and her contributions to science, law, and academic freedom (pp. 35-63). New York: Psychology Press.

Zaragoza, M. S., Lane, S M., Ackil, J. K., \& Chambers, K. L. (1997). Confusing real and suggested memories: Source monitoring and eyewitness suggestibility. In N. L. Stein, P. A. Ornstein, B. Tversky, \& C. Brainerd (Eds.), Memory for everyday and emotional events (pp. 401-425). Mahwah, NJ: Erlbaum.

Zeighami, R., Behnammoghadam, M., Moradi, M., \& Bashti, S. (2018). Comparison of the effect of eye movement desensitization reprocessing and cognitive behavioral therapy on anxiety in patients with myocardial infarction. European Journal of Psychiatry, 32(2), 72-76. https://doi.org/10.1016/j.ejpsy.2017.09.001

Zhu, B., Chen, C., Loftus, E. F., Lin, C., He, Q., Chen, C., ... \& Dong, Q. (2010). Individual differences in false memory from misinformation: Cognitive factors. Memory, 18(5), 543-555. https://doi.org/10.1080/09658211.2010.487051 



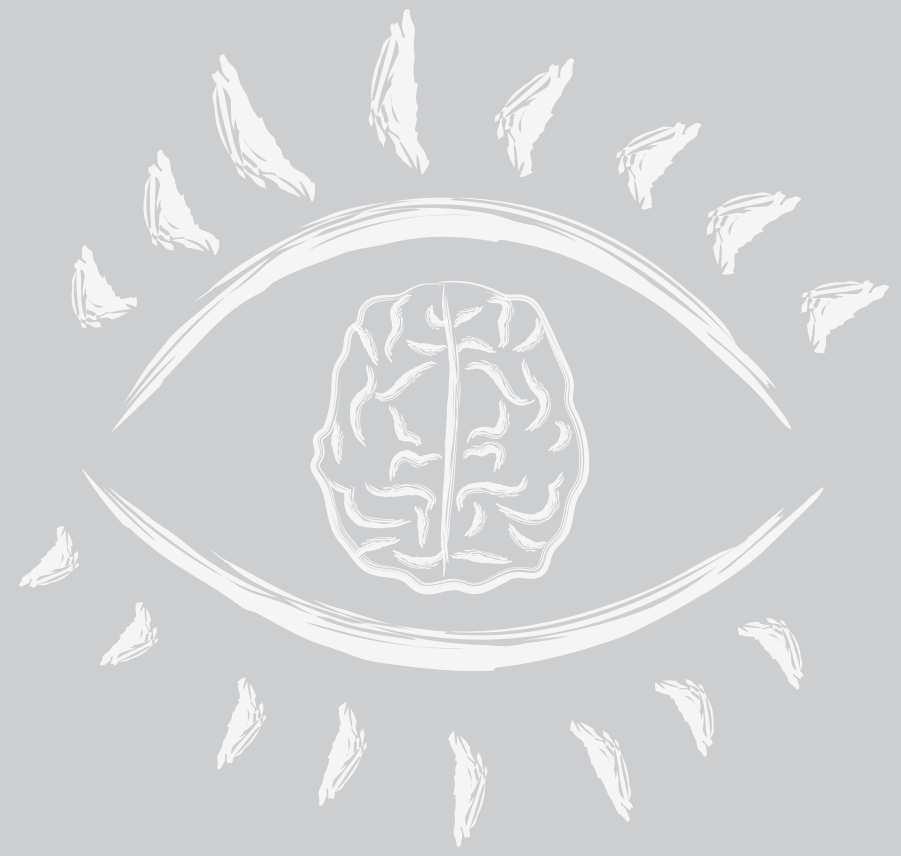


Dankwoord 

Time flies when you are having fun! De afgelopen jaren hebben meerdere mensen mij geholpen dit proefschrift af te ronden en daarom wil ik hen graag bedanken voor hun steun. Ik ben vast iemand vergeten, maar dat is dan alleen in woorden, niet in gedachten.

Als eerste wil ik mijn begeleiders bedanken. Henry, tijdens het tweede jaar van mijn bacheloropleiding psychologie heb je me laten zien hoe leuk onderzoek is. Je passie voor onderzoek werkt aanstekelijk en ik heb veel van je geleerd. Ik wil je bedanken voor je begeleiding de afgelopen jaren en op naar nog meerdere samenwerkingen! Jeffrey, ik bewonder je combinatie van je eigen praktijk, onderzoek, opleiding tot klinisch psycholoog, en gezin. Ik wil je bedanken voor je interesse in de persoon áchter de promovendus. Je staat altijd voor me klaar. Jouw steun en de ruimte die je me gaf om te groeien als persoon, en daarmee ook als onderzoeker, waardeer ik enorm! Harald, om jou in een aantal woorden te bedanken is haast onmogelijk. Gezien je drukke agenda wilde ik je nooit lang storen, maar afspraak of niet: als ik binnenliep had je alle aandacht en tijd voor me. Je wetenschappelijke kennis, enthousiasme, scherpe opmerkingen op mijn manuscripten, en de steun van begin tot eind is waar menig professor wat van kan leren. Ik kan je niet genoeg bedanken voor de vrijheid en het vertrouwen die je me hebt gegeven om de onderzoeker te worden die ik nu ben. Ik hoop nog meer van je te leren te komende jaren!

De leden van de beoordelingscommissie en de corona: Hartelijk dank voor jullie tijd om mijn proefschrift te lezen en beoordelen.

Daarnaast wil ik ook mijn coauteurs bedanken. Peter Muris, Lawrence Patihis, Eric Rassin, en Tom Smeets: I learned a lot from all of you. Your different perspectives and (critical) feedback improved my manuscripts, thanks for your collaboration! Brechje Dandachi-FitzGerald en Ineke Wessel, naast coauteur heb ik ook het genoegen gehad om meer intensief met jullie samen te werken. Ik wil jullie bedanken voor jullie betrokkenheid tijdens mijn promotietraject en hoop dat ik nog vaak met jullie mag samenwerken in de toekomst!

Mijn paranimfen, Martijn en Glynis, bedankt dat jullie mij bij staan op deze bijzondere dag! Martijn, m'n chocolade buddy, vanaf dag één heb je me onder je hoede genomen en ik kon met alles bij je terecht. Ik ga je ontzettend missen, maar ook al scheiden onze wegen qua werk, ben ik ervan overtuigd dat onze band sterk genoeg is om te blijven bestaan. Dank voor 
al jouw steun! Glynis, van docent en student naar collega's. Gedurende de jaren heb ik je vaak om raad kunnen vragen, keurde je mijn presentaties en posters en zijn we een sterk team bij het onderwijs dat we samen verzorgen. Aan onze gezichtsuitdrukkingen weten we vaak al genoeg. De knuffels (en bueno's) om onze leuke nieuwtjes te vieren halen we in! Dank dat je er altijd voor me bent!

Graag wil ik ook alle collega's van de forensische psychologie sectie en de klinische psychologie sectie in Maastricht en LINC en CELL in Leuven bedanken. Dank voor alle gezellige lunches, vergaderingen, retraites, en congressen!

Een paar (ex-) collega's wil ik in het bijzonder bedanken. Anna en Conny, samen met Glynis vormen jullie 'my three wise women'. Anna, thanks for your wise words and advice! Conny, het is soms akelig hoe veel wij op elkaar lijken. Je bent een echte die hard en ik kan altijd bij je terecht. Dank voor alles! Carey, Bruna (my lobster), and Elly ( $m$ 'n horror lover), thanks for all the laughter and good (loooong) talks! Nina, I finally found someone who loves Christmas (and Doritos) as much as I do. Irena, maybe because of the size of KKW's butt it took us so long to finally connect and to bond over the important things in life. I owe you big time!

Ook wil ik graag mijn roomies bedanken voor de gezellige jaren. Danique, Eline, Elly, Eveliina, Kristoff, Linda, Martijn, Nina, and Tameka: Thank you for tolerating my laughs, frustrations, the long talks about literally everything, being my guinea pigs for my baking creatures, and accepting my Christmas music from September onwards. My roomies from Leuven: Fabiana, Ivan, Jane, Nael, and Paul: thank you for the great times!

Caroline, Daniëlle, Jessie, Lindy, Marionne, en Paula: Dank voor al jullie goede zorgen en luisterende oren!

Graag wil ik ook mijn familie bedanken. Dank voor al jullie warmte, interesse, steun, en onvoorwaardelijke liefde. Met jullie als familie is het leven gewoon leuker! Esther, Marijn, Nicolle, Raf, en Roos (mijn grenzeloze paranimf): allen zo verschillend, maar een vriendschap die ik koester! Dank voor de alle leuke uitjes, gesprekken, en er gewoonweg voor mij te zijn! 
Papa, mama, en Thomas: zonder jullie was dit allemaal niet mogelijk. De zelfstandigheid, assertiviteit, en mensen in hun waarde laten (maar tegelijkertijd ook kunnen piekeren als de beste): het zijn allemaal eigenschappen waarin jullie me een basis hebben gegeven en waarin ik de afgelopen jaren erg in ben gegroeid. Dank voor al jullie steun, ongeacht wat ik doe.

Mijn grootste steun en toeverlaat, mijn grote liefde Thomas. Nadat ik jou heb mogen ondersteunen tijdens jouw promotietraject, was het nu jouw beurt. Jij was mijn oase van rust als ik die nodig had. Door jouw advies en engelengeduld heb ik mijn promoveren op de best mogelijke manier kunnen doorlopen. Je bent er altijd voor me en ik ben zo gelukkig met een partner zoals jou. We zijn samen zo veel gegroeid. Wat wij samen hebben, kan niemand ons afnemen. Ik houd van jou! 


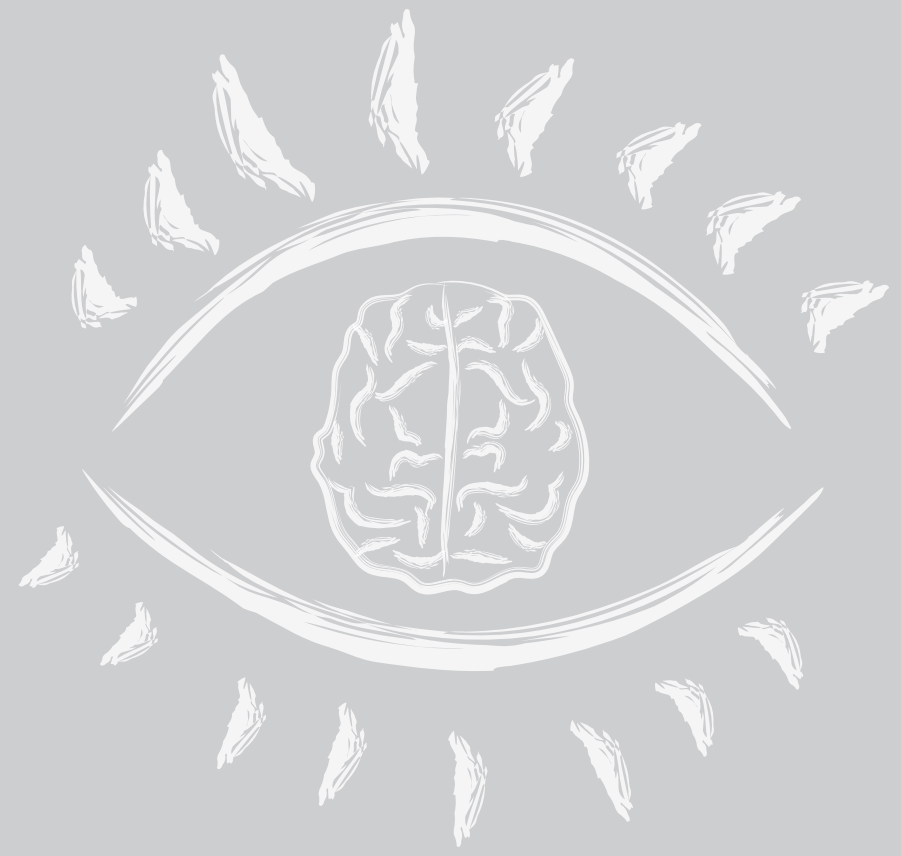


Curriculum vitae 

Sanne Houben werd geboren op 12 november 1992 te Maastricht. In 2011 behaalde zij haar vwo-diploma aan het Porta Mosana College te Maastricht. In september van dat jaar begon zij aan haar studie Psychologie aan de Universiteit Maastricht, waarna ze in 2015 haar masterdiploma in Psychology and Law behaalde. Vanaf januari 2017 werd zij aan de Faculteit der Psychologie en Neurowetenschappen van de Universiteit Maastricht aangesteld als promovendus voor onderzoek naar de vatbaarheid voor pseudoherinneringen bij therapieën zoals Eye Movement Desensitization and Reprocessing. In 2017 ontving Sanne een travel grant om deel te nemen aan een summer school in Weggis, Zwitserland. In 2019 voltooide zij haar basis kwalificatie onderwijs.

Sanne Houben was born on November 12, 1992 in Maastricht. In 2011, she graduated from secondary school at Porta Mosana College, Maastricht. In September of that year, she started studying Psychology at Maastricht University, after which she received her masters' diploma in Psychology and Law in 2015. From January 2017, she was appointed at the Faculty of Psychology and Neuroscience of Maastricht University as a PhD student on the susceptibility to false memories from psychological interventions such as Eye Movement Desensitization and Reprocessing. In 2017, Sanne received a travel grant to attend a summer school in Weggis, Switzerland. In 2019, she completed the university teaching qualification. 


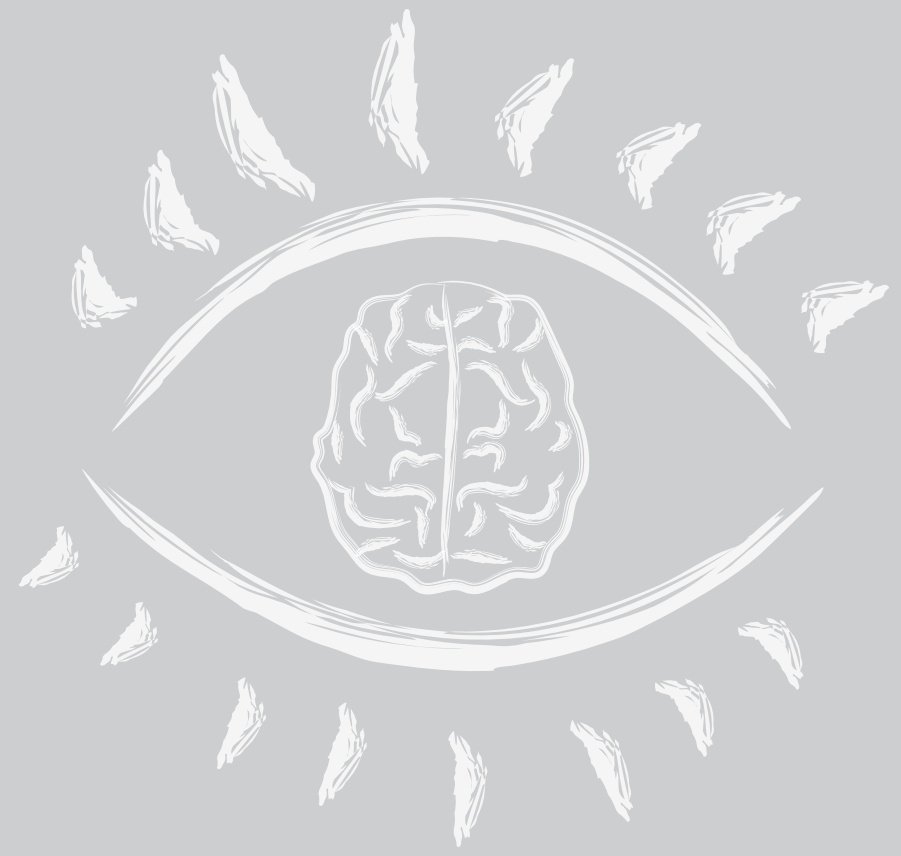


List of publications 



\section{INTERNATIONAL JOURNAL PUBLICATIONS}

Houben, S. T. L., Otgaar, H., \& Roelofs, J. (in press). Psychological myths as therapeutic instruction in EMDR. The Journal of Psychology: Interdisciplinary and Applied.

Houben, S. T. L., Otgaar, H., Roelofs, J., Merckelbach, H., \& Muris, P. (2020). The effects of eye movements and alternative dual tasks on the vividness and emotionality of autobiographical memory: A meta-analysis. Journal of Experimental Psychopathology. https://doi.org/10.1177/2043808720907744

Houben, S. T. L., Otgaar, H., Roelofs, J., Smeets, T., \& Merckelbach, H. (2020). Increases of correct memories and spontaneous false memories due to eye movements when memories are retrieved after a time delay. Behavior Research and Therapy, 125, 103546. https://doi.org/10.1016/j.brat.2019.103536

Houben, S. T. L., Otgaar, H., Roelofs, J., Wessel, I., Patihis, L., \& Merckelbach, H. (2019). Eye movement desensitization and reprocessing (EMDR) practitioners' beliefs about memory. Psychology of Consciousness: Theory, Research, and Practice. Advance online publication. https://doi.org/10.1037/cns0000211

Houben, S. T. L., Otgaar, H., Roelofs, J., \& Merckelbach, H. (2019). EMDR and False Memory: A response to Lee, de Jongh, and Hase (2019). Clinical Psychological Science, 7(3), 405-406. https://doi.org/10.1177/2167702619830392.

Houben, S. T. L., Otgaar, H., Roelofs, J., \& Merckelbach, H. (2018). Lateral eye movements increase false memory rates. Clinical Psychological Science, 6(4), 610-616. https://doi.org/10.1177/2167702618757658

Houben, S. T. L. (2016). 'That didn't happen': Memorial Consequences of False Denials for Neutral and Negative Materials. Maastricht Student Journal of Psychology and Neuroscience, 5, 9-24.

\section{DUTCH JOURNAL PUBLICATIONS}

Houben, S. T. L., Otgaar, H., Rassin, E., \& Merckelbach, H. (2020). Getuigen die Eye Movement Desensitization and Reprocessing (EMDR) ondergaan: Een riskante osmose tussen psychologie en recht [Witnesses undergoing Eye Movement Desensitization and Reprocessing (EMDR): A risky osmosis between psychology and law]. Expertise en Recht, 4, 119-123. 
List of publications

Houben, S. T. L. (2020). Tinder voor psychotherapie? Over de blinde vlek van therapeuten [Tinder for psychotherapy? About the blind spot of therapists]. De Psycholoog, 55, 10-18. (Publication prize)

Houben, S. T. L. (2019). Lichaam onthoudt wat geest wil vergeten [The body remembers what the mind wants to forget]. De Psycholoog, 6, 28.

Merckelbach, H., Houben, S. T. L., Dandachi-FitzGerald, B., Otgaar, H., \& Roelofs, J. (2018). Als psychotherapie faalt [When psychotherapy fails]. De Psycholoog, 10, 10-21.

\section{BOOK CHAPTERS}

Otgaar, H., \& Houben, S. T. L. (in press). Pseudoherinneringen [False memories]. In: H. Otgaar, H. Merckelbach, \& M. Jelicic (Eds.), Rechtspsychologie. Paris Uitgeverij.

Otgaar, H., Houben, S. T. L., \& Howe, M. L. (2019). Methods of studying false memory. In: B.L. Schwartz \& H. Otani, (Eds.), Research methods in human memory research (pp. 238-250). New York, NY: Routledge.

\section{UNDER REVIEW}

Otgaar, H., Schell-Leugers, J. M., Howe, M. L., De La Fuente Vilar, A., Houben, S. T. L., \& Merckelbach, H. (in revision). Individual differences in false confessions: A review using experimental, field, and case studies. Applied Cognitive Psychology.

\section{ORAL AND POSTER PRESENTATIONS}

Houben, S. T. L. (2021, July). Increases of correct memories and spontaneous false memories due to eye movements after time delay. Presentation at the $32^{\text {nd }}$ International Congress of Psychology in Prague, Czech Republic.

Houben, S. T. L. (2021, July). Memory wars 2.0? EMDR practitioners' beliefs about memory. Poster presentation at the $32^{\text {nd }}$ International Congress of Psychology in Prague, Czech Republic.

Houben, S. T. L. (2021, June). Memory wars 2.0? EMDR practitioners' beliefs about memory. Poster presentation at the EMDR Europe Research \& Practice Conference in Dublin, Ireland. 
Houben, S. T. L., et al. (2020, November). Effects of EMDR on (false) memory. Zoom Psychology and Law Symposium.

Houben, S. T. L., et al. (2020, November). Psychologische metaforen als therapeutische instructie in EMDR [Psychological metaphors as therapeutic instructions in EMDR]. Symposium organised at the Vereniging Gedrags en Cognitieve Therapieën (VGCT) najaarscongres, Zoom.

Houben, S. T. L. (2020, July). De geheugeneffecten van oogbewegingen: Klinische implicaties [The memory effects of eye movements: Clinical implications]. Invited talk at Via Icarus, Cadier en Keer, the Netherlands.

Houben, S. T. L. (2020, June). Do your eyes protect your memory? The susceptibility to spontaneous false memories after performing eye movements as used in EMDR. Talking about Memory and Cognition, Zoom.

Houben, S. T. L. (2020, February). The memory effects of EMDR and therapy side effects. Invited talk at Helmut Schmidt University, Hamburg, Germany.

Houben, S. T. L. (2020, February). The memory effects of EMDR and therapy side effects. Invited talk at University Medical Center Hamburg-Eppendorf, Hamburg, Germany.

Houben, S. T. L., et al. (2019, November). Kennis van EMDR therapeuten over de werking van het geheugen [EMDR therapists' knowledge on the functioning of memory]. Presentation at the Vereniging Gedrags en Cognitieve Therapieën (VGCT) najaarscongres in Veldhoven, the Netherlands.

Houben, S. T. L. (2019, October). Do your eyes protect your memory? The memory effects of EMDR. Presentation at the symposium of Experimental Psychopathology EPP in Heeze, the Netherlands.

Houben, S. T. L., et al. (2019, June). The effects of eye movements on the susceptibility to spontaneous false memories. Symposium organised at the $13^{\text {th }}$ Society for Applied Research in Memory and Cognition in Cape Cod, United States.

Houben, S. T. L., et al. (2019, April). Memory wars 2.0? EMDR practitioners' beliefs about memory. Poster presentation at the FPN research day in Maastricht, the Netherlands. (Best poster presentation)

Houben, S. T. L., et al. (2019, April). Zo spontaan! Het geheugeneffect van oogbewegingen [So spontaneous! The memory effect of eye movements]. Presentation at the $12^{\text {th }}$ EMDR conference of Vereniging EMDR Nederland in Utrecht, the Netherlands.

Houben, S. T. L. (2019, February). De geheugeneffecten van EMDR [Memory effects of EMDR]. 
List of publications

Presentation at Lucertis/Youz in Maastricht, the Netherlands.

Houben, S. T. L., et al. (2018, June). Lateral eye movements increase false memory rates. Presentation at the 29th International Congress of Applied Psychology (ICAP) in Montréal, Canada.

Houben, S. T. L., et al. (2018, June). A potential drawback of EMDR. Presentation at the Swiss Graduate School of Cognition, Learning, and Memory in Weggis, Switzerland.

Houben, S. T. L., et al. (2018, May). Therapists' beliefs about memory. Presentation at the Maastricht \& Memory symposium in Maastricht, the Netherlands.

Houben, S. T. L., et al. (2018, April). Drawback of EMDR? Pitch presentation at the FPN Research day in Maastricht, the Netherlands.

Houben, S. T. L., et al. (2018, April). Geheugenillusies in de maak: Over het geheugenvertekend effect van oogbewegingen [Memory illusion in the making: About the memory distorted effect of eye movements]. Presentation at the $11^{\text {th }}$ EMDR conference of Vereniging EMDR Nederland in Utrecht, the Netherlands.

Houben, S. T. L., et al. (2018, April). Drawback of EMDR. Pitch presentation at the Experimental Psychopathology EPP day in Utrecht, the Netherlands.

Houben, S. T. L., et al. (2017, October). Lateral eye movements increase false memory rates. Presentation at the University of Maastricht Faculty of Law Annual Graduate Studies Conference: The World of Interdisciplinary Research in Maastricht, the Netherlands.

Houben, S. T. L., et al. (2017, May). The false memory effects of EMDR. Pitch presentation at the $27^{\text {th }}$ European Association of Psychology and Law Conference (EAPL) in Mechelen, Belgium.

Houben, S. T. L., et al. (2017, March). The false memory effects of EMDR. Pitch presentation at the Experimental Psychopathology EPP day in Utrecht, the Netherlands.

\section{(Best pitch presentation)}

\section{MEDIA}

Houben, S. T. L. (2020, February). Rechtspsycholoog waarschuwt: EMDR-therapie kan ook pseudoherinnering opvissen [Legal psychologist warns: EMDR therapy can evoke false memories]. Interview retrieved from Trouw at https://www.trouw.nl/ binnenland/rechtspsycholoog-waarschuwt-emdr-therapie-kan-ook -pseudoherinnering-opvissen $\sim$ b1624c4c 
Houben, S. T. L. (2020, February). EMDR kan leiden tot pseudoherinneringen [EMDR can lead to false memories]. Interview retrieved from Nederlandstalige Vereniging voor Psychotrauma: https://www.ntvp.nl/ntvp-interview-sanne-houben-emdr-kan-leiden -tot-pseudoherinneringen

Houben, S. T. L. (2020, January). Traumabehandeling en pseudoherinneringen [Traumatherapy and false memories]. Interview retrieved from Radio 1: https://radio1.be/programma/ nieuwe-feiten/radioitem/valse-herinneringen/11332

Houben, S. T. L. (2020, January). Traumatherapie lokt pseudoherinneringen uit [ Traumatherapy evokes false memories]. Interview retrieved from EOS Wetenschap, 1, 8. https:// www.eoswetenschap.eu/psyche-brein/traumatherapie-lokt-pseudoherinneringen-uit Houben, S. T. L., et al. (2020, January). Overtuigd van verdrongen herinneringen? [Convinced of repressed memories?]. News article retrieved from Vereniging Gedrags en Cognitieve Therapieën (VGCT) at https://www.vgct.nl/themas/trauma/-ptss/ overtuigd-van-verdrongen-herinneringen

Houben, S. T. L. (2020, January). Junior-Sanne Houben. Interview retrieved from De Psycholoog, 55, 20-21. https://www.tijdschriftdepsycholoog.nl/artikelen/sanne-houben Houben, S. T. L. (2019, October). De rode vlaggen van therapie: Hervonden herinneringen cursus voor therapeuten [Red flags of therapy: Recovered memories course for therapists]. Blog retrieved from https://flpmaastrict.org/blog/blog-post-one-a7pzr

Houben, S. T. L. (2019, September). Oogbewegingen en pseudoherinneringen [Eye movements and false memories]. Vlog retrieved from https://youtube.com/ watch?v=ZDxGQFIZFjg\&t=2s

\section{GRANTS AND AWARDS}

- $\quad$ Publication prize for young authors awarded by De Psycholoog (2019, November).

- Best poster award at Faculty of Psychology and Neuroscience faculty day (2019, April).

- Travel grant for summer school of the Swiss graduate school for cognition, learning, and memory (2017, December).

- First place at the "Battle of the Brains" - Research PhD pitches Experimental Psychopathology (2017, March). 